\title{
Phase diagrams of two-dimensional frustrated spin systems

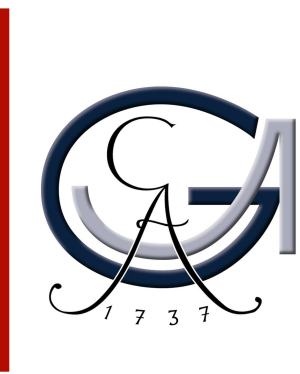

\section{Dissertation}

zur Erlangung des mathematisch-naturwissenschaftlichen Doktorgrades „Doctor rerum naturalium“ der Georg-August-Universität zu Göttingen

vorgelegt von Ansgar Kalz aus Berlin

Göttingen 2012 
Referent: Prof. Dr. Reiner Kree

Korreferent: PD Dr. Andreas Honecker

Korreferent: Prof. Stefan Wessel, Ph.D.

Tag der mündlichen Prüfung: 22. März 2012 


\section{acknowledgment/Dank}

An dieser Stelle möchte ich mich bei all denen bedanken, die mich während der Promotion und bei der Fertigstellung dieser Doktorarbeit unterstützt haben. Insbesondere gilt mein herzlichster Dank Andreas Honecker, der mir dieses Promotionsvorhaben ermöglicht hat, mir stets mit Rat und Tat zur Seite stand und mich in die internationale Forschungsgemeinschaft integriert hat.

Die endgültige Version dieser Arbeit verdankt ihre Form auch den vielen hilfreichen Korrekturen und Kommentaren meiner Bürokollegen Peter Wächter (bis 2010), Piet Dargel und Oliver Bodensiek, und einer gründlichen Revision der englischen Fassung durch Jonathan Fish, ein herzliches Dankeschön an alle vier. Des Weiteren möchte ich mich bei Thomas Pruschke und der gesamten Arbeitsgruppe für rege (physikalische) Diskussionen in Seminaren und bei Kaffee und Kuchen bedanken.

Ein spezieller Dank gilt auch meiner Mutter Cornelia Kalz, meinem Vater Wilfried Kalz und seiner Frau Verena Richter, meiner Schwester Bernadette Kalz und meiner Freundin Christina Thiede, die mich immer ermutigt und unterstützt haben während der gesamten Zeit meiner Promotion.

Also I would like to thank my further scientific collaborators for their efforts and the great cooperation in the different projects over the past years, namely Marion Moliner from Karlsruhe (chapter 5), Gennady Chitov from Sudbury in Canada (chapter 6), Sebastian Fuchs from Göttingen (chapter 7) and Marcelo Arlego, Daniel Cabra and Gerardo Rossini from La Plata in Argentina (chapter 8). In addition I appreciate the work of the authors of the ALPS project ${ }^{a}$ (in particular Sebastian Fuchs) and the Spinpack package $^{b}$ (Jörg Schulenburg). Furthermore, I would like to thank Jürgen Holm, the $\mathrm{GWDG}^{c}$ and the $\mathrm{HLRN}^{d}$ for technical support during the past years. Last but not least I thank the DFG for financial support via the SFB 602 Komplexe Strukturen in kondensierter Materie ${ }^{e}$ (TP A18) and the DAAD for a short term scholarship (Grant No. D/10/46833) which enabled me to visit my collaborators in Argentina (09-10/2010).

Ansgar Kalz, Februar 2012

\footnotetext{
$a_{\text {http: //alps.comp-phys.org }}$

${ }^{b}$ http://www-e.uni-magdeburg.de/jschulen/spin/

${ }^{c}$ http://www.gwdg.de

$d_{\text {http: }} / /$ www $\cdot$ hlrn.de

ehttp://www . uni-goettingen.de/de/103216.html
} 


\section{authors note}

At the end of this thesis a complete list of all references with detailed information (all authors, title, url) is given in alphabetical order of the labels. The labels for all references are constructed by the initials of up to four authors of the work and a two-digit number representing the year of publication or appearance. If more than one reference is cited at the same location the labels are ordered alphabetically to make it easier to find them all in the list at the end rather than in chronological order.

The references which were published within this thesis are:

[KHM11] Kalz A., Honecker A. and Moliner M., Analysis of the phase transition for the Ising model on the frustrated square lattice. Physical Review B, 84, 174407 (2011), URL http://dx.doi. org/10.1103/PhysRevB.84.174407.

[KHFP11a] Kalz A., Honecker A., Fuchs S. and Pruschke T., Quantum disordered ground state for hard-core bosons on the frustrated square lattice (+ Erratum Phys. Rev. B 84, 219902(E) (2011)). Physical Review B, 83, 174519 (2011), URL http://dx.doi.org/10. 1103/PhysRevB.83.174519.

[KHFP11b] Kalz A., Honecker A., Fuchs S. and Pruschke T., Quantum disordered ground state for the frustrated square lattice. proceedings of SCES 2011, preprint: 1110.3162 (2011), URL http://arxiv . org/abs/1110.3162.

$\left[\mathrm{KAC}^{+} 12\right]$ Kalz A., Arlego M., Cabra D., Honecker A. and Rossini G., Anisotropic frustrated Heisenberg model on the honeycomb lattice. Physical Review B, 85, 104505 (2012), URL http://dx. doi.org/10.1103/PhysRevB.85.104505. 


\section{table of contents}

chapter 1 introduction 1

1.1. ordering processes in the $2 \mathrm{D}$ Ising model . . . . . . . . . . . . . . . . 2

1.2. disorder by quantum fluctuations . . . . . . . . . . . . . . 5 5

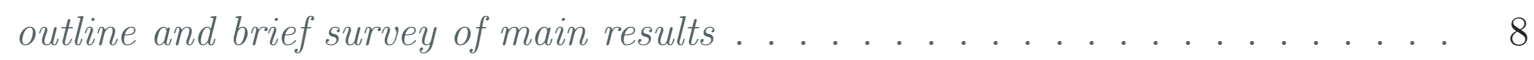

chapter 2 interacting spin systems in two dimensions 9

2.1. statistical mechanics . . . . . . . . . . . . . . . . . . . . . . 9 9

2.1.1. thermodynamics . . . . . . . . . . . . . . . 10

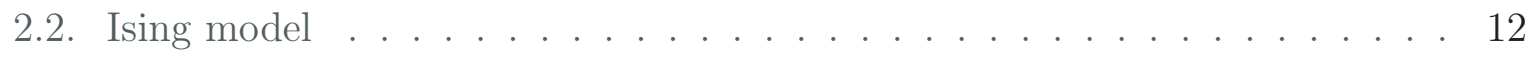

2.2.1. ground states . . . . . . . . . . . . . . . . . . . . 12

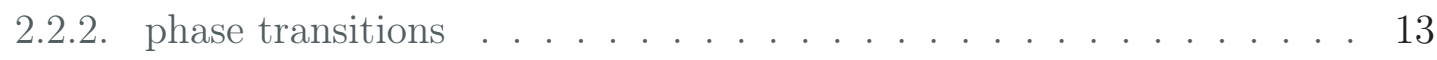

2.3. Heisenberg model . . . . . . . . . . . . . . . . . . . . 15

2.3.1. hard-core bosons . . . . . . . . . . . . . . . . 16

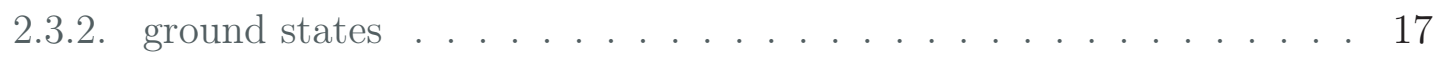

$\begin{array}{lll}\text { chapter } 3 & \text { computational methods } & 21\end{array}$

3.1. exact diagonalization . . . . . . . . . . . . . . . . . . . 21

3.1.1. symmetries . . . . . . . . . . . . . . . . 22

3.1.2. algorithms . . . . . . . . . . . . . . . . . . . 23

3.2. Monte-Carlo simulations . . . . . . . . . . . . . . . . . . . 23

3.2.1. classical Monte-Carlo algorithm . . . . . . . . . . . . . . 24

3.2.2. quantum Monte-Carlo algorithm ... . . . . . . . . . . . . . 25

3.2.3. observables . . . . . . . . . . . . . . . . . . . . . 28

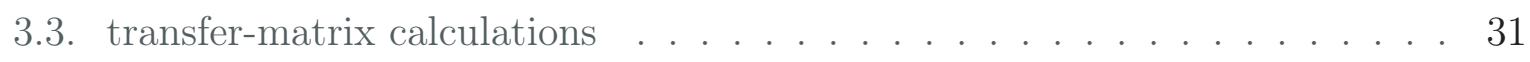

chapter 4 analytical methods 33

4.1. Ising series expansion for weak quantum fluctuations . . . . . . . . . . . 33

4.2. conformal field theory . . . . . . . . . . . . . . . 34

side note: conformal transformations . . . . . . . . . . . . . . 35

4.2.1. conformal field theory for a statistical model . . . . . . . . . 35

4.2.2. perturbative renormalization . . . . . . . . . . 36

4.2.3. operator product expansion . . . . . . . . . . . . . . . . . . . . . . . . . . . . .

4.2.4. statistical models . . . . . . . . . . . . . . . . . 38 


\section{I ordering processes in the 2D Ising model}

chapter 5 analysis of the phase transition for the Ising model on the frustrated square lattice $\quad 41$

5.1. Monte-Carlo simulation . . . . . . . . . . . . . . . . . . . . . . . 42

5.1.1. phase transition analysis . . . . . . . . . . . . . . . . . . . . . . . . . . . . . . . 43

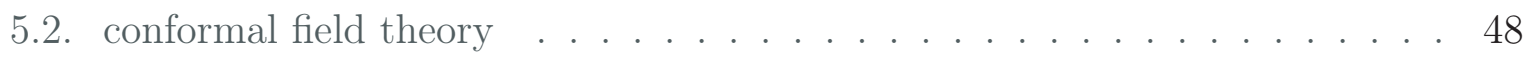

5.2.1. transfer matrix . . . . . . . . . . . . . . . . . 48

5.2.2. continuum limit . . . . . . . . . . . . . . . . . . 49

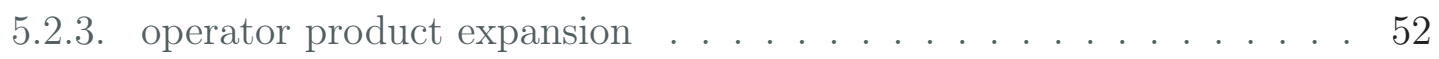

5.2.4. Ashkin-Teller model . . . . . . . . . . . . . . . . . 54

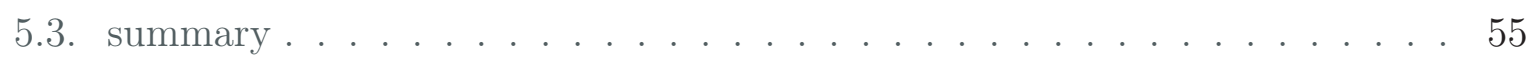

chapter 6 incommensurate ordering in a spatially anisotropic Ising $\begin{array}{ll}\text { model } & 57\end{array}$

6.1. phase diagram . . . . . . . . . . . . . . . . . . . . . . . . . . 58

6.2. energy and specific heat . . . . . . . . . . . . . . . . 58

6.3. order parameter and correlation functions ............. 61

6.4. summary . . . . . . . . . . . . . . . . . . . . . 65

\section{II disorder by quantum fluctuations}

chapter 7 quantum disordered ground state for hard-core bosons on $\begin{array}{ll}\text { the frustrated square lattice } & 69\end{array}$

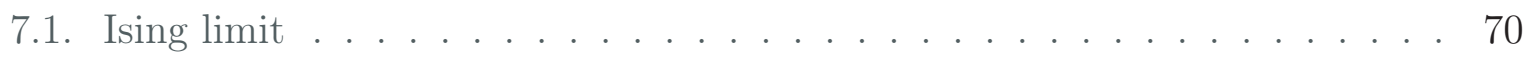

7.2. ferromagnetic limit . . . . . . . . . . . . . . . . . . . . . . . . . . . . . . . . . . . . . . . .

7.3. intermediate regime . . . . . . . . . . . . . 76

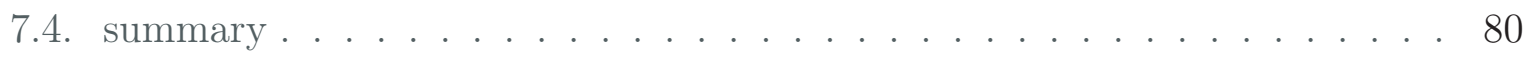

chapter 8 anisotropic frustrated Heisenberg model on the honeycomb $\begin{array}{ll}\text { lattice } & 81\end{array}$

8.1. Ising limit . . . . . . . . . . . . . . . . . . . . . . . . . . 82

8.2. ferromagnetic limit . . . . . . . . . . . . . . . . . . . 84

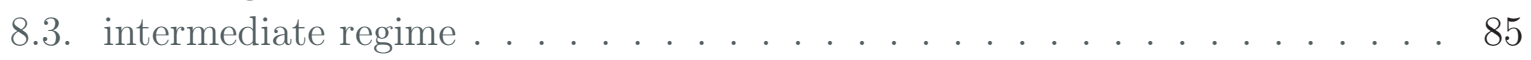

8.4. summary . . . . . . . . . . . . . . . . . . . . . . . . . . 88

$\begin{array}{lll}\text { chapter } 9 & \text { concluding remarks } & 91\end{array}$

9.1. phase transitions in the Ising model . . . . . . . . . . . . . . . . . . 91

9.2. quantum disordered ground states . . . . . . . . . . . . . . . . . 93

9.3. future prospects . . . . . . . . . . . . . . . . . . . . . 95 


\section{appendices}

chapter A computational details

A.1. classical Monte-Carlo

A.2. quantum Monte-Carlo ..................... III

A.3. transfer-matrix implementation . . . . . . . . . . . . . IV

chapter B conformal field theory $\quad$ VII

B.1. continuum limit derivations . . . . . . . . . . . . . . . . VII

B.2. operator product expansion . . . . . . . . . . . . IX

B.2.1. second order perturbation .............. . . X

B.2.2. integration of prefactors .................. XI

B.2.3. third order calculations .................. XII

\section{list of references}




\section{introduction $\mid 1$}

Shortly after the discovery of High- $T_{C}$ superconductivity in the copper compound $B a-$ $L a-C u-O$ in 1986 [BM86] Phil Anderson proposed an insulating quantum disordered state as basis for a new mechanism of superconductivity [And87]. In the abstract of his paper he wrote:

"This insulating phase is proposed to be the long-sought "resonating-valencebond" state or "quantum spin liquid" hypothesized in 1973.» (introduced by Anderson [And73] as well)

Since then many classes of materials, e.g., heavy-fermion materials [SS10, Ste84, even before the cuprates], ${ }^{1}$ iron-based compounds [CEE08], and organic composites [LWB ${ }^{+} 00$, McK97] have shown superconductivity at comparably high temperatures $\left(T_{C}>20 \mathrm{~K}\right)$. However, from the theoretical point of view, despite many efforts, there is still no final answer to the question of the true nature of such unconventionally superconducting states [And92, And97, LNW06, Nor11, Sac12, ZCS ${ }^{+}$06]. Quite the contrary, it is clear nowadays that no single mechanism is responsible for the superconductivity in different types of High- $T_{C}$ materials. In Copper compounds showing superconductivity two features are very prominent.

Firstly the undoped compounds are insulating [And97]. This fact has motivated the research on quantum spin models since the 1980s [LMM11, ML05, RSH04]. These models of strongly correlated electrons are defined in the insulating state of matter and only the spin degrees of freedom of the electrons contribute. The focus lies thus on the magnetic properties of the material rather than on its electronic character.

Secondly a strong spatial anisotropy both in the chemical structure and the physical properties is observed; i.e., the materials consist of two-dimensional layers and also the magnetic and electronic characteristics exhibit the same spatial anisotropy [And97]. In quantum spin models the dimensionality also plays a crucial role in the context of ordering processes. The conditions under which a quantum spin liquid most likely exists, were characterized by Anderson [And87]:

«This insulating magnetic phase is favored by low spin, low dimensionality, and magnetic frustration.»

The quantum spin liquid is a superposition of quantum mechanically entangled states without any long-range order [And73]. This quantum entanglement is mediated by quantum spin fluctuations of the corresponding spins which may exceed the classical magnetic interactions in particular for small spins and in low-dimensional models [RSH04, Sac04]. The smallest unit is a quantum entangled pair of spins (a singlet) which, according to Anderson, becomes the charge carrier in the superconducting phase if the insulator is doped sufficiently [And87]. In the insulating phase, these singlets cover the lattice and can be

\footnotetext{
${ }^{1}$ To any literature cited in the this chapter, the abbreviation 'e.g.' and the comment 'plus references therein' should be added, as articles, reviews and books listed may have good substitutes.
} 
arranged in a regular configuration giving rise to quantum long-range order. However, the absence of long-range order in the spin-liquid state even for $T \searrow 0$ makes the detection and validation of such a disordered ground state very complicated. No local symmetry is broken and, hence, no local order parameter exists for this phase [Gol93]. According to the Mermin-Wagner theorem [MW66] in isotropic models (i.e., quantum fluctuations and classical interactions are scaled with the same parameter) a long-range ordered ground state in two dimensions can only be stable at zero temperature. At higher temperatures the order is destroyed by thermal and quantum fluctuations. By breaking the isotropy or taking the classical limit of the quantum models long-range order can be established at finite temperatures [Joy67, KT73]. For dominating homogeneous magnetic interactions - either ferromagnetic or antiferromagnetic - the system is forced into a long-range magnetically ordered state [RSH04]. However, the introduction of competing interactions between different spins by involving, for example, magnetic exchange on longer distances results in frustration. I.e., there is no global configuration of spins that satisfies all local magnetic interactions [Die05]. For frustrated systems the importance of quantum fluctuations is thus enhanced again and may lead to the emergence of quantum ordered or disordered states [Bal10, $\left.\mathrm{BBB}^{+} 05, \mathrm{ML05}\right]$.

The case of two-dimensional quantum spin liquids has in particular attracted a lot of interest in recent decades, because of its relation to topological ordered states [JYB11, KL87, ML05, Wen91]. The order for these states does not only depend on the internal parameters but also on the topology of the system, e.g., boundary conditions, shape, and size of the system. The topological order cannot be verified by a local order parameter, neither, and therefore the spin liquid is a promising candidate to represent such a state. Topologically ordered states and especially their excitations are discussed as possible candidates for the implementation of so called qubits, i.e., quantum bits that serve as the smallest unit of a quantum computer [LMSS12, OP99].

Motivated by these two major topics of modern condensed matter physics, i.e., High$T_{C}$ superconductivity and quantum computation, the search for quantum spin liquids has become a very active field since the 1990s, both experimentally [CTTT01, ONAKT07, $\mathrm{SMK}^{+}$03] and theoretically [CL98, MS01]. Promising candidates to exhibit a spin-liquid ground state from the theoretical point of view are spin-1/2 Heisenberg models in frustrated two-dimensional systems [JYB11, WGWV11, YHW11]. One focus of this thesis is a detailed analysis of the ground-state phase diagrams of a two-dimensional frustrated quantum Heisenberg model with anisotropic exchange parameters on the square and honeycomb lattice (more details in section 1.2).

A good starting point for exploring the physics of the quantum-mechanical spin models is a deeper understanding of the underlying classical spin models that are obtained by taking the limit of zero quantum fluctuations. The ground states of these frustrated Ising models are easily identified, but the ordering processes and phase transitions are still subject of ongoing research and will be the topic of chapters 5 and 6 of this thesis.

\section{ordering processes in the 2D Ising model ${ }^{2} \mid 1.1$}

The simple Ising model which adds up the interactions of two-state variables on a $D$ dimensional lattice has served as a pioneer in many physical problems, especially in sta-

\footnotetext{
${ }^{2}$ Some results of this part are published in [KHM11] and the following introduction is partially based on this manuscript.
} 
tistical mechanics and solid-state physics [Isi25, Len20]. It was one of the first models to mimic the magnetic exchange interactions in condensed-matter theory, and the behavior of phase transitions has been studied extensively for this model in different dimensions and by numerous approaches. It was solved for the one-dimensional chain by Ising [Isi25] and for the square lattice with nearest-neighbor interaction by Onsager [Ons44]. In two dimensions the model with nearest-neighbor interactions $J_{1}$ undergoes an ordering process at a finite temperature which is well understood and establishes the Ising universality class for second-order phase transitions [Bax82, Gol93, Ons44, see also next chapter].

However, for additional frustrating interactions $J_{2}$ on next-nearest neighbor bonds the phase diagram of the model becomes richer and the physics of the occurring phase transitions becomes more complicated. These complications originate from the competition of different ground states that occur for certain parameters in the phase diagram. At these critical points a large degeneracy of macroscopic order often arises and this hampers the analysis of the system [KHFP08].

A fully analytic solution of the frustrated $J_{1^{-}} J_{2}$ model has not been found. However, the behavior of the model in the vicinity of the phase transition can be analyzed by applying different numerical and analytic techniques, e.g., renormalization-group transformations [Bax82, Car96], Monte-Carlo simulations [Ber04, LB05, see also section 3.2.1], transfer-matrix calculations [Bax82, section 3.3] for the lattice model and mean field [Gol93], and conformal field theory [dFMS97, Mus10, section 4.2] for the continuous version of the model. With the exception of renormalization and mean-field techniques, all of these methods will be applied in this thesis and will be introduced in chapters 3 and 4. Monte-Carlo simulations in particular have been very successful for the investigation of the Ising model [KHFP08, KHFP09, LB05] and will serve as primary method in this work. However, Monte-Carlo simulations suffer severe thermalization problems due to the ground-state degeneracy in the vicinity of the critical point. To overcome these problems in this thesis an additional exchange Monte-Carlo step [Han97, HN96, KTHT06] is introduced. For this purpose, sets of simulations with the same parameters (system size, exchange parameters) are computed simultaneously at different temperatures, and according to a probability function, which depends on the temperature and the energy of the system, the spin configurations of different simulations can be exchanged. Because of the possible large changes in the configuration each simulation thermalizes more rapidly; the algorithm is also referred to as parallel tempering since many simulations are thermalized parallelly.

Using this improved algorithm two cases are studied in the context of this work: firstly an isotropic version of the model, where all next-nearest neighbor interactions are set to the same constant value $J_{2}$, and secondly an anisotropic version where two different values for the next-nearest neighbor coupling $J_{2}^{a}=-J_{2}^{b}$ are used.

\section{isotropic Ising model}

In chapter 5 the focus lies on the phase transition from the high-temperature paramagnetic phase to an antiferromagnetic collinear phase that is favored by an isotropic antiferromagnetic coupling $J_{2}$ on next-nearest neighbor bonds in the square lattice, i.e., $J_{2}>J_{1} / 2$. This transition has attracted a lot of interest in the past. In the late 1970s renormalizationgroup calculations and Monte-Carlo simulations for the Ising model with frustrating interactions were performed by Nightingale [Nig77], Swendsen \& Krinsky [SK79], and later on by Oitmaa [Oit81] as well as by Landau \& Binder [BL80, Lan80, LB85, LB05]. For this particular model they assumed a continuous phase transition and computed tran- 
sition temperatures and critical exponents. Throughout the 1980s it was commonly accepted that the exponents are weakly universal but vary for different degrees of frustration [Bax82, page 254], i.e., only the scaling relations for the exponents are fulfilled but the absolute values are not universal.

A different point of view is adopted by describing the corresponding continuous model using conformal field theory [CS09, dFMS97, Mus10, see also section 4.2]. This field theory is characterized by a central charge $c$ which can be calculated via the free energy of the system [Aff86, BCN86]. However, a continuous phase transition with non-universal exponents is only possible if the central charge of the underlying conformal field theory is $c \geq 1$ [FQS84]. Meanwhile theories with discrete $c<1$ define universality classes with universal exponents such as the two-dimensional Ising model $(c=1 / 2)$ [BPZ84a, BPZ84b]. The frustrated $J_{1}-J_{2}$ Ising model can be described by two identical independent copies of nearest-neighbor $J_{2}$ Ising models at the point $J_{1}=0$ with a total central charge of $c=1$. Furthermore, for increasing $J_{1}>0$ a decrease of the central charge is expected according to the $c$ theorem [Zam86], and hence, the universality of the phase transition was under debate. In 1993 López et al. presented a mean-field calculation for the model [MLAGS93, MLAGS94] where they find a first-order transition for a finite parameter region of $0.5<J_{2} / J_{1} \lesssim 1.1$. Recent Monte-Carlo simulations by the group of Malakis et al. contradict this scenario at least for the value $J_{2} / J_{1}=1$ [MKT06]. On the other hand, Monte-Carlo results in published in [KHFP08, KHFP09] strengthen the argument of a first-order transition for small values of $0.5<J_{2} / J_{1} \leq 0.7$.

Using Monte-Carlo simulations it will be shown in the chapter 5 that the region of validity for the first-order transition is increased up to $J_{2} \lesssim 0.9 J_{1}$. However, the MonteCarlo simulations do not give a conclusive picture for larger values of $J_{2}$ due to increasing length scales. Hence, it was necessary to apply further techniques to understand the nature of the phase transition for these parameters. To compute the central charge of the model a finite-size analysis will be performed for the free energy which was calculated via transfer-matrix techniques. In a last step the limit of two decoupled Ising models with antiferromagnetic coupling $J_{2}$ is assumed and an antiferromagnetic nearest-neighbor interaction $J_{1}$ is added perturbatively between the two copies. In second-order perturbation theory an Ashkin-Teller model is obtained which is in agreement with a scenario of non-universal exponents.

\section{anisotropic Ising model}

For the anisotropic case, where $J_{2}$ is not the same for all next-nearest neighbor bonds, the phase diagram of the Ising model on the square lattice exhibits an additional ground state for ferromagnetic interactions $J_{2}^{a}$ along one direction of diagonal couplings and antiferromagnetic interactions $J_{2}^{b}=-J_{2}^{a}$ along the perpendicular direction [CG05]. The same state was earlier described by Landau and Binder for the axial-next-nearest neighbor Ising model (ANNNI) [LB85]. The finite-temperature phase transition to this ground state was discussed by Chitov and Gros using mean-field arguments [CG05]. They predict an intermediate phase that exhibits incommensurate ordering, i.e., the smallest unit of a set of spins that show a regular alignment is incompatible with the system size for this phase. Therefore, the ordering process and the topology of the system are closely connected for these states and the concept of topological excitations is discussed in that context [Bak82].

The concept of incommensurate phases and phase transitions from incommensurate to commensurate ordered states is also discussed in different versions of the Ising model in two and three dimensions, see for example [Bak82, BvB80, MI11, Sel88, SM99, VB81, 
ZC11]. In the same context the scenario of floating phases, those that are characterized by different order parameters and combine different commensurate phases, is described in [Bak82] for two-dimensional systems. The incremental change of the corresponding ordering wave vector (see section 2.1) with an external parameter such as temperature or coupling is often referred to as the devil's staircase [Bak82]. The number of steps depends not only on the systems size but also on the remaining parameters, and thus the staircase reflects the intrinsic properties of the system. This devil's staircase has also been observed experimentally (see, e.g., $\left[\mathrm{FML}^{+} 78\right]$ ).

In chapter 6 Monte-Carlo results of the anisotropic Ising model will be presented for energies, correlation functions and their Fourier transform - the structure factor. Strong evidence of a floating phase within the anisotropic two-dimensional Ising model is given which has not been observed before. The results are compared to the predictions of Chitov and Gros [CG05] and it is shown that their calculations for the ordering wave vector yield an upper limit for the staircase of ordering vectors observed in the simulations.

\section{\begin{tabular}{l|l} 
disorder by quantum fluctuations $^{3}$ & 1.2
\end{tabular}}

By introducing quantum fluctuations into the classical Ising model and keeping the discrete values of the spin variables at the same time the quantum mechanical Heisenberg model is defined [Hei28]. A first solution was given by Bethe [Bet31] for the one-dimensional spin- $1 / 2$ chain. Already in two dimensions no analytic solution has been found. To study the ground-state behavior of such a two-dimensional quantum spin model a variety of methods are applicable in principle, e.g., exact diagonalization [BF64, LP04, OB78, see section 3.1], perturbative methods like series expansion [Kog79, OHZ06, see section 4.1] or linear spin waves [HP40, IS04], the coupled cluster method [BLDR08, FB04] or the density matrix renormalization group and its further developments [Sch11, Whi92] (all at $T=0$ ), and quantum Monte-Carlo [SK91, see also section 3.2.2] (at finite temperature). Each method exhibits its advantages and drawbacks such as, for example, the limitation on accessible system sizes (exact diagonalization, variational methods) or the uncertainty of the underlying assumptions (perturbative methods, some variational methods). The major drawback of the quantum Monte-Carlo approach is the sign-problem ${ }^{4}$ which is severe for completely frustrated spin models. However, by lifting the frustration for some magnetic interactions and accepting the loss of isotropy of the original spin model, the sign problem can be avoided and statistically exact results can be obtained for reasonably large systems. The quantum spin models studied in this work are anisotropic, i.e., the quantum fluctuations stem from ferromagnetic interactions, and can be simulated without the sign problem. These spin models can then be interpreted alternatively as models of hard-core bosons [MM56] with non-frustrating kinetic energy and repulsive interaction on a two-dimensional lattice (a more detailed description is given in section 2.3). The same perspective was adopted in earlier works for several frustrated lattice problems [BS00, CMWK08, $\left.\mathrm{HBS}^{+} 01, \mathrm{NC} 08\right]$. However, the particular models studied in this work include hopping and interactions on all considered bonds, and they have not been analyzed previously at half-filling.

\footnotetext{
${ }^{3}$ Most results of this part are published in $\left[\mathrm{KAC}^{+} 12\right.$, KHFP11a, KHFP11b] and the following introduction is based on these manuscripts.

${ }^{4}$ This special problem of quantum Monte-Carlo simulations is explained in section 3.2.2.
} 
Similarly to the classical case the remaining frustration for the repulsive interactions of the model leads to thermalization problems in the quantum Monte-Carlo simulations which can be overcome in the same manner by introducing an exchange Monte-Carlo step, whereby the probability distribution for the acceptance of the exchange step has to be adopted to the quantum Monte-Carlo algorithm [SSC02, Mel07].

The bosonic interpretation also opens a new possibility to compare theoretical results to experimental realizations of the model on optical lattices [ $\left.\mathrm{LSA}^{+} 07, \mathrm{SOL}^{+} 11\right]$. These artificial perfect lattices are constructed by standing waves of laser beams and can be used as simulators for quantum mechanical models of interacting particles. The mechanism works particularly well for bosonic systems. However, a construction of lattices with frustrating interactions has only been achieved recently $\left[\mathrm{JGT}^{+} 12\right]$ and needs further investigation.

In the quantum spin model for limiting cases of small and large quantum fluctuations magnetic phases are expected to be stable: antiferromagnetic states resemble the Ising ground states for small quantum fluctuations and a ferromagnetic long-range correlation in the perpendicular plane for large quantum fluctuations. In bosonic language, the antiferromagnetic states are described by boson-density waves. The ferromagnetic inplane order is interpreted as Bose condensation of the magnons and hence corresponds to a superfluid order in the bosonic model [Blo32, Hoe50, LF73, MM56]. A combination of both is referred to as supersolid and has been discussed in previous works for similar models [CMWK08, HD05, LF73, MPB ${ }^{+} 05, \mathrm{NC08}, \mathrm{WT05}$ ]. The name originates in the bosonic description of the model where this kind of ordering combines superfluid order with density-wave order. Apart from the magnetic phases the emergence of a disordered spin-liquid state (or resonating valence bond) and a variety of quantum phases showing long-range order is discussed for quantum spin models $\left[\mathrm{BBB}^{+} 05\right]$. The configurations of such states are given, e.g., by a parallel alignment of singlets which represent a quantum mechanically entangled state of two spins (see, for example, figure 2.5 on page 19). These singlets or valence bonds can be either arranged in a fixed regular configuration yielding valence bond solids or the lattice is covered by a dynamic disordered assembling which is described by different singlet configurations (hence the name resonating valence bond). The search for these quantum states in frustrated quantum spin models on the square and honeycomb lattice is the subject of chapters 7 and 8 of this thesis.

\section{square lattice}

One of the most interesting and challenging problems in the field of frustrated quantum spin models is the $J_{1^{-}} J_{2}$ spin-1/2 isotropic Heisenberg model on the bipartite square lattice [BLDR08, CBPS03, ML05, MLPM06, OW96, RDS ${ }^{+}$10, RMP09, RS09, RSH04]. In the vicinity of the point of highest frustration a variety of phases is discussed and recently evidence for a spin-liquid ground state was given in [JYB11, WGWV11]. As described above, non-variational quantum Monte-Carlo simulations have a severe sign problem for the frustrated model and are thus very limited for such a system. In chapter 7 a bosonic model which maps onto the Heisenberg model for a certain set of parameters is investigated and may give some crucial hints for the completely frustrated model. For similar models, quantum ordered phases have been predicted $\left[\mathrm{BBB}^{+} 05\right]$.

Starting from the classical model without quantum fluctuations, which is analyzed in chapter 5 , the quantum model is examined for finite temperatures and the quantum Monte-Carlo results are extrapolated to $T=0$ to form a ground-state phase diagram. For the equivalent anisotropic Heisenberg model, two classical antiferromagnetically ordered 
phases (Néel and collinear state) are found to be stable as ground states for small quantum fluctuations. A direct transition between these two antiferromagnetic configurations is verified by means of quantum Monte-Carlo simulations and series expansion. For large fluctuations the system becomes ferromagnetic in the $x y$ plane. Close to the highly frustrated point, which is accompanied by a large ground-state degeneracy in the classical limit, a region with no finite order parameter is identified and this state is interpreted as quantum disordered after excluding long-range dimer order by additional Monte-Carlo calculations for higher correlation functions [San92]. Furthermore, the low-energy spectrum of a small lattice is calculated via exact diagonalization to exclude topological order in the disordered phase of the ground-state phase diagram [ML05, MLMS02].

\section{honeycomb lattice}

The honeycomb lattice has received much attention in the past years because of its electronic structure, which gives rise to interesting behavior observed in graphene [CGP $\left.{ }^{+} 09\right]$. However, this two-dimensional bipartite lattice with its two-site unit cell was investigated long before it was realized in a real material. It is particularly interesting for quantum mechanical models of strongly correlated electrons, since its coordination number $n=3$ is the lowest allowed in a two-dimensional system [FSL01, RSH04]. Hence, the influence of quantum fluctuations on the ground state properties is expected to be more important than, for example, in the also bipartite square lattice. Recently, it was found that the Hubbard model on the honeycomb lattice exhibits a spin liquid state at the edge of the metal-insulator transition for an intermediate value of the onsite repulsion [CAS11, $\left.\mathrm{MLW}^{+} 10\right]$. Since then the investigation of spin models that can be derived perturbatively from the Hubbard model [YLMS10, YS11], or stated directly inside the insulating phase, has yielded many interesting features. The ground-state phase diagrams include disordered and valence bond solid phases for different parameters [ASH ${ }^{+} 11$, CLR11, MB12, MGCP10, MR11, RAT11]. Furthermore, in the context of heavy-fermion physics, Iridium compounds have been investigated that can be described by frustrated spin models on the honeycomb lattice and exhibit antiferromagnetic order [KY11].

In chapter 8 a spin model on the honeycomb lattice is investigated, including nearest, next-nearest and third-nearest neighbor anisotropic Heisenberg interactions, a geometry analyzed in previous works for the isotropic Heisenberg model $\left[\mathrm{ASH}^{+} 11\right.$, CLR11, FSL01, RAT11]. The starting point is again the limit of small fluctuations, where $S^{z}$ interactions along a preferred direction are all antiferromagnetic, leading to frustration. The quantum fluctuations in the transversal plane are non-frustrating and, hence, ferromagnetic as for the square lattice. Thus, the predictions for possible ground states are very similar and indeed, the phase diagram obtained by quantum Monte-Carlo simulations in chapter 8 resembles the latter case qualitatively. A stability estimation of the several phase boundaries calculated by linear spin wave theory and series expansion reproduces the numerical results very well. On a quantitative level, the region without any magnetic order differs from the square lattice model, and again the calculation of higher correlation functions does not provide any evidence for a long-range ordered quantum phase. 
outline and brief survey of main results

chapter 2: interacting spin systems in two dimensions

introduction of the investigated spin models, their main properties and a short outline of the applied framework of statistical mechanics

chapter 3: computational methods

exact diagonalization, (quantum) Monte-Carlo simulations and transfer matrix technique

chapter 4: analytical methods

series expansion and conformal field theory

chapter 5: analysis of the phase transition for the Ising model on the frustrated square lattice [KHM11]

evidence for first-order transition for a finite region of parameters (Monte-Carlo), perturbative derivation of the Ashkin-Teller field theory from the frustrated Ising model giving rise to non-universal critical behavior for the remaining parameters (conformal field theory)

chapter 6: incommensurate ordering in a spatially anisotropic Ising model verification of an incommensurate phase between the paramagnetic and antiferromagnetic phase in the anisotropic Ising model, detection of several finite signals in the structure factor, a floating phase resembling a devil's staircase is identified (Monte-Carlo)

chapter 7: quantum disordered ground state for hard-core bosons on the frustrated square lattice [KHFP11a, KHFP11b]

analysis of the stability of magnetic phases (series expansion and quantum Monte-Carlo), finite region without any long-range order is detected (quantum Monte-Carlo), topological order is excluded (exact diagonalization)

chapter 8: anisotropic frustrated Heisenberg model on the honeycomb lattice $\left[\mathrm{KAC}^{+} 12\right]$

determination of magnetic phase boundaries (quantum Monte-Carlo) and comparison to perturbative results (series expansion and linear spin waves), detection and analysis of intermediate disordered state (quantum Monte-Carlo and exact diagonalization)

chapter 9: concluding remarks

concluding discussion of results and brief survey of future prospects 


\section{interacting spin systems in two dimensions}

\section{summary}

In section 2.1 of this chapter some crucial concepts of statistical mechanics are repeated. These will be used to describe the spin models and to analyze the physical properties of them. For this work the classical Ising model and the quantum mechanical Heisenberg model are of particular interest and will be introduced in sections 2.2 and 2.3, respectively. For the Ising model ground-state configurations and phase-transition scenarios are outlined for the frustrated square lattice. The Heisenberg model is defined as spin and boson system on the square and honeycomb lattice, and possible ground states are discussed.

\section{\begin{tabular}{l|l} 
& \\
statistical mechanics & 2.1
\end{tabular}}

The framework for analyzing the theoretical models that will be introduced in the following sections is the theory of statistical mechanics, because the number of particles and hence the number of available states is generally extremely large ${ }^{1}$. The ensemble concept allows for the derivation of thermodynamic properties on the basis of all accessible microstates that contribute for a certain set of parameters [Sch00]. Throughout the present work the temperature $T$ and the number of particles $N$ is fixed for each calculation, ${ }^{2}$ which are the prerequisites to work in the canonical ensemble. The value of interest for a model defined by its Hamiltonian $H$ will then be the canonical partition function

$$
Z_{\text {can. }}=\operatorname{Tr} \exp (-\beta H)=\sum_{k} \exp \left(-\beta E_{k}\right), \quad \beta=\frac{1}{k_{B} T},
$$

which sums over the Boltzmann weights of all states $k$ at the inverse temperature ${ }^{3} \beta$. The representation of $Z_{\text {can. }}$ given above requires: (a) a discrete spectrum of energies which is always the case for the discrete spin models that are the subject of this thesis and (b) the knowledge of all eigen-energies $E_{k}$ of the system. For quantum systems the derivation of these eigen-energies will be a challenging task, confer section 3.1 on page 21.

\footnotetext{
${ }^{1}$ Usually at this point the Avogadro constant is introduced which defines the number of particles in an amount of one mol of material to be in the order of $N \sim 10^{23}$. However, the maximal number of spins that will be taken into account in this work is much lower $N \sim 10^{6}$. Nevertheless, due to the exponential growth of states with $N$ this task is already quite challenging with today's computer power.

${ }^{2}$ The word calculation refers to the attempt to extract concrete data from the model for certain parameters. Hence, it is mostly a computational approach - a simulation - and only in some cases actual analytic calculations will be performed.

${ }^{3}$ In the following the Boltzmann constant will be set $k_{B}=1$, as well as the Planck constant $\hbar=1$. However, it is important to keep in mind that $\beta$ actually represents an inverse energy.
} 


\section{\begin{tabular}{l|l} 
thermodynamics & 2.1 .1
\end{tabular}}

The expectation value of an observable $A$ using the partition function is given by

$$
\langle A\rangle=\frac{1}{Z_{\text {can. }}} \sum_{k} A_{k} \exp \left(-\beta E_{k}\right) .
$$

The internal energy ${ }^{4} E=\langle E\rangle$ (and the entropy $S$ ) can be deduced from the thermodynamical potential $F$ which defines the free energy

$$
F=E-T S=-\frac{1}{\beta} \ln Z_{\text {can. }} \rightarrow E=\frac{\partial(\beta F)}{\partial \beta}=-\frac{\partial Z_{\text {can. }}}{\partial \beta} .
$$

The free energy is the corresponding thermodynamic potential and it connects the concept of statistical mechanics with the thermodynamic observables. All thermodynamic observables can be derived from it using Legendre transformations and further derivatives [Sch00]. Furthermore, the concept of phase transitions, which will be explained briefly below, is connected with the behavior of the free energy.

\section{order parameter}

To detect and distinguish different phases of a system that exhibit different local symmetries, observables are introduced that are sensitive to the ordering processes that happen at a phase transition [Gol93, Sch00]. For spin models these local order parameters are connected with the internal magnetic properties of the system. The spin-spin correlation functions $S_{i} S_{j}$ can be used to track different long-range ordered configurations in the lattice by taking the Fourier transform at different momenta $\mathbf{q}$ - the spin structure factor [Mah00]:

$$
S(\mathbf{q})=\frac{1}{N} \sum_{i, j} \exp \left(\mathbf{q}\left(\mathbf{r}_{i}-\mathbf{r}_{j}\right)\right) S_{i} S_{j}, \quad \mathbf{r}_{i} \text { : position of } S_{i}
$$

Each momentum vector $\mathbf{q}$ classifies a different spin configuration, e.g., for $\mathbf{q}=(0,0)$ a ferromagnetic alignment of all spins gives a maximal signal in the structure factor which in that case resembles the magnetization

$$
\mathbf{q}=(0,0): m_{\text {ferro. }}=\sqrt{\frac{S(\mathbf{q})}{N}}=\frac{\sqrt{\sum_{i, j} S_{i} S_{j}}}{N}=\frac{1}{N} \sqrt{\sum_{i} S_{i} \sum_{j} S_{j}}=\frac{1}{N} \sum_{i} S_{i} .
$$

The order parameter is chosen such that it gives a finite signal in the corresponding ordered phase and equals zero for the remaining region of the phase diagram [Gol93]. The calculation of higher moments and cumulants of these order parameters plays a crucial role for the accurate analysis of the phase transitions.

\section{phase transitions}

A thermodynamical system aims to be in a state of minimal energy and maximal entropy; the combination of both is given by the free energy as shown in equation (2.3). The

\footnotetext{
${ }^{4}$ Throughout this work the notion of an observable will always refer to the expectation value of this observable if not stated otherwise explicitly.
} 
importance of the maximization of the entropy decreases with temperature and, hence, a state with higher order and less symmetry that minimizes the internal energy is favored as a ground state. This symmetry breaking manifests itself in the free energy as a phase transition at a certain critical temperature $T_{C}$; the characteristics of the free energy and its derivatives in the vicinity of that critical point classify the phase transition. If the free energy $F$ shows non-analytic behavior at $T \approx T_{C}$ the phase transition is not continuous but of first-order. The energy gap stemming from the discontinuity is called latent heat and manifests itself in a two-peaked structure in the energy distribution at the critical point. This feature can be observed in energy histograms and will be used in chapter 5 to verify a first-order phase transition in the two-dimensional Ising model.

For continuous transitions the scaling of $F$ with the reduced temperature $\tau=\frac{T-T_{C}}{T_{C}}$ can be described by continuous power laws which also applies for the order parameter, correlation functions and other observables, for more details see [Car96, Gol93, Sch00]. The result of a scaling analysis is a set of critical exponents for these power laws. Critical exponents are universal for classes of phase transitions that share similar properties: the symmetry of the order parameter, the local number of states, the dimensionality and the range of the interactions define different universality classes. One of the most important magnitudes for the scaling analysis is the correlation length $\xi$ which scales with the exponent $\nu$ and diverges at the critical temperature. Because the divergence in a finite system is limited, a finite-size analysis is inevitable for the investigation of critical behavior on finite lattice systems. However, this same analysis can be used to extract $\nu$ and the remaining critical exponents. A further feature of the critical exponents can be derived by going back to their origin; the scaling of the free energy imposes scaling (and in some cases hyper-scaling) relations on the critical exponents, i.e., they are not independent and have to fulfill certain relations [Car96, Gol93]. At this point the concept of continuously varying exponents may be introduced. There are continuous phase transitions with critical exponents that obey these scaling relations but vary continuously. The ratios of the exponents with $\nu$ are universal again [Bax82]. Hence, these transitions sometimes are referred to as weak-universal [Suz74].

The individual exponents specify the scaling for the order parameter $(\beta)$, its susceptibility $(\gamma)$, the specific heat $(\alpha)$, the correlation functions $(\eta)$ and the corresponding correlation length $(\nu)$. Hence, higher moments of the order parameter $m$ scale with multiples of $\beta$ and in particular the fourth order cumulant

$$
U_{4}=\frac{3}{2}\left(1-\frac{\left\langle m^{4}\right\rangle}{3\left\langle m^{2}\right\rangle^{2}}\right), \quad \text { where } \quad U_{4}\left(T=T_{C}\right) \propto L^{0},
$$

gives the same value for different system sizes $L \times L$ at the critical temperature, i.e., $T_{C}$ can be determined from the single intersection point of the cumulants, as was pointed out by Binder [Bin81a, Bin81b]. The cumulant $U_{4}$ is chosen such that the signal in the ordered phase tends towards a finite constant value ${ }^{5}$ and in the disordered phase the signal equals zero. This behavior originates in the distinct distribution of the order parameter in the ordered and disordered phase and the particular prefactors given in equation (2.6).

Furthermore, fluctuations of the order parameter and the energy are enhanced in the vicinity of the critical point and, thus, the susceptibility $\chi$ the specific heat $C$ are good indicators for phase transitions:

$$
\chi=\frac{\left\langle m^{2}\right\rangle-\langle m\rangle^{2}}{T} \text { and } C_{V}=\frac{\left\langle E^{2}\right\rangle-\langle E\rangle^{2}}{T^{2}} .
$$

\footnotetext{
${ }^{5}$ The constant value depends on the model, the order parameter, and the normalization.
} 


\section{\begin{tabular}{l|l} 
Ising model & 2.2
\end{tabular}}

The basic assumptions of the Ising model are that every site of the lattice is occupied by a two-state spin variable ${ }^{6} S_{i}= \pm 1$ and that spins on different sites interact only pairwise - with ferromagnetic $\left(J_{i, j}<0\right)$ or antiferromagnetic exchange $\left(J_{i, j}>0\right)$ - and with an optional magnetic field $h_{i}$. The Hamiltonian and, hence, the energy of the system is given by summing over all possible interactions and sites, respectively:

$$
H_{\text {Ising,gen. }}=\sum_{i \neq j} J_{i, j} S_{i} S_{j}+\sum_{i} h_{i} S_{i} .
$$

In the following the magnetic field will always be zero and the interaction strength $J_{i, j}$ will be fixed to a homogeneous value for a given distance of the sites $i$ and $j$. In particular, the focus will be on the two-dimensional square lattice with $N=L \times L$ sites and interactions $J_{1}$ for all nearest neighbors $(\mathrm{NN})$ and $J_{2}^{i, j}$ for next-nearest neighbors (NNN):

$$
H_{\text {Ising,square }}=J_{1} \sum_{\mathrm{NN}} S_{i} S_{j}+\sum_{\mathrm{NNN}} J_{2}^{i, j} S_{i} S_{j} .
$$

For antiferromagnetic interactions on the next-nearest neighbor bonds in the square lattice the model is frustrated, and no global configuration minimizes all interactions contributing to the Hamiltonian.

In chapter 5 both $J_{1}>0$ and $J_{2}>0$ will be chosen antiferromagnetic whereas in chapter 6 the coupling $J_{2}$ will be anisotropic, i.e., ferromagnetic in one direction (connecting next-nearest neighbors) and antiferromagnetic in the perpendicular direction. In both cases the focus will be on analyzing the phase transition for dominating next-nearest neighbor interactions $J_{2}$.

\section{\begin{tabular}{l|l} 
ground states & 2.2 .1
\end{tabular}}

For high temperatures - compared to the energy scale $T \gg J_{1} \sim J_{2}$ - the spins on different sites are nearly independent because thermal fluctuations overrule the potential energy of the interactions and the system is in a disordered paramagnetic state. At lower temperatures the system minimizes its overall energy by aligning spins (anti-)parallel on nearest and/or next-nearest neighbor bonds, respectively. In the non-frustrated nearest-neighbor Ising model $\left(J_{2}=0\right)$ a (anti)-ferromagnetic ground state is stable in two dimensions. For isotropic antiferromagnetic $J_{1}>0$ and $J_{2}>0$ two ground states with a total magnetization of $M=\left\langle\sum_{i} S_{i}\right\rangle=0$ are obtained: a Néel ordered state (figure 2.1 (a) left) and a collinear ordered state (figure 2.1 (a) right). A comparison of both ground state energies

$$
E_{\text {Néel }}=-2 N\left(J_{1}-J_{2}\right) \text { and } \quad E_{\text {coll }}=-2 N J_{2}
$$

yields a critical point at $J_{2}=J_{1} / 2$. The ground state at this point is largely degenerate and this degeneracy has a strong influence on the physics in the vicinity of the critical

\footnotetext{
${ }^{6}$ The absolute value of $S_{i}$ has no influence on the physical properties of the model, rather it fixes the energy scale of the system.
} 
Néel order

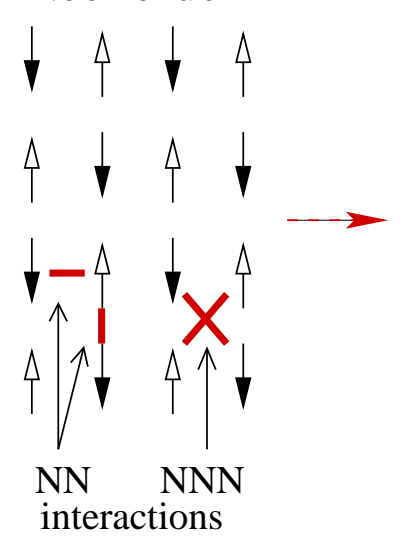

collinear order

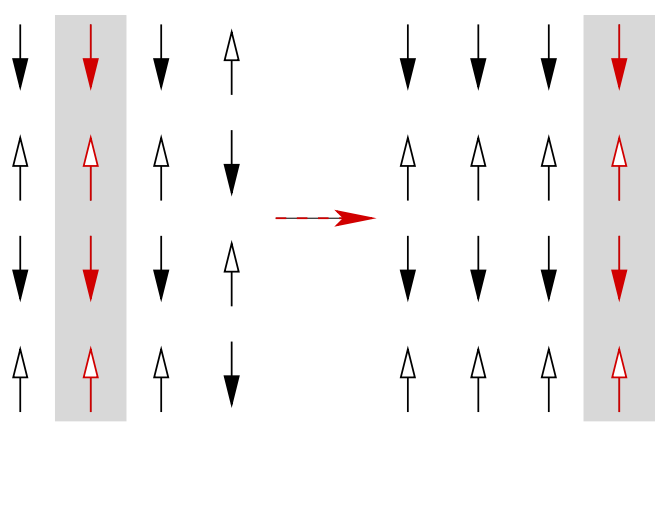

(a) isotropic model superferro-antiferromag.

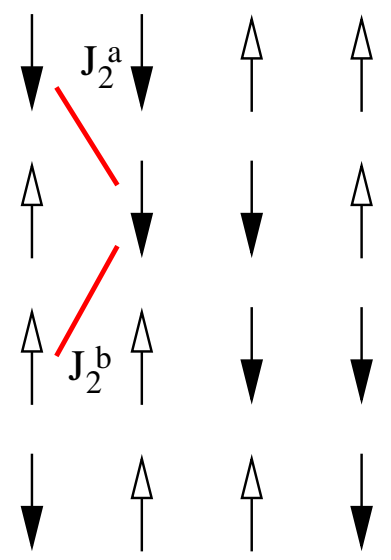

(b) anisotropic model

figure 2.1: (a) Shown on the left hand side is the Néel ordered state for a $4 \times 4$ square lattice, on the right hand side the collinear configuration. In the middle a transition state is shown which is part of the degenerate ground state manifold of the frustrated model at the critical point (sketch taken from [Kal08, p. 6]). (b) Ground state for the anisotropic model for $2\left|J_{1}\right|<\left|J_{2}^{a}\right|+\left|J_{2}^{b}\right|$.

point, see [Kal08, KHFP08, KHFP09]. Appropriate order parameters for these states are given by the spin structure factor (see section 2.1) for specific momenta:

$$
\text { Néel order: } \mathbf{q}=(\pi, \pi) \quad m_{\text {Néel }}=\frac{\sqrt{S(\mathbf{q})}}{N}=\frac{1}{N} \sum_{i}(-1)^{r_{i}^{x}+r_{i}^{y}} S_{i}
$$

collinear order: $\mathbf{q}=(\pi, 0) \wedge(0, \pi)$

$$
m_{\text {coll. }, x}=\frac{1}{N} \sum_{i}(-1)^{r_{i}^{x}} S_{i} \wedge m_{\text {coll. }, y}=\frac{1}{N} \sum_{i}(-1)^{r_{i}^{y}} S_{i} .
$$

The latter is a combination of two different order parameters measuring the configurations that differ by an angle of $90^{\circ}$.

For an anisotropic choice of $J_{2}^{i, j}$, i.e., ferromagnetic in one direction $\left(J_{2}^{a}<0\right)$ and antiferromagnetic in the perpendicular direction $\left(J_{2}^{b}>0\right)$ another ground state consisting of ferro- and antiferromagnetic structure arises (shown in figure 2.1(b) and called superferro-antiferromagnetic state - SFAF) and competes with a Néel state (for $J_{1}>0$ ):

$$
E_{\mathrm{Néel}}=-N\left(2 J_{1}+J_{2}^{a}-J_{2}^{b}\right) \quad \text { and } \quad E_{\mathrm{SFAF}}=-N\left(J_{2}^{a}+J_{2}^{b}\right) .
$$

The critical point is given by $2 J_{1}=J_{2}^{b}-J_{2}^{a}$ and in the special case of $-J_{2}^{a}=J_{2}^{b}$ this is reduced to $J_{1}=J_{2}^{b}$. The ordering wave vector is $q=(\pi / 2, \pi / 2)$.

Equivalently, for a negative (ferromagnetic) $J_{1}$ the competing state is the ferromagnetic ground state for both models.

$$
\text { phase transitions } \mid 2.2 .2
$$

For the non-frustrated case $\left(J_{2}=0\right)$ the critical temperature $T_{C}=2 J_{1} \operatorname{arsinh}^{-1}(1) \approx$ $2.269 J_{1}$ can be calculated exactly [Ons44] and the phase transition between the paramagnetic phase and the magnetically ordered state is a continuous one with well defined 


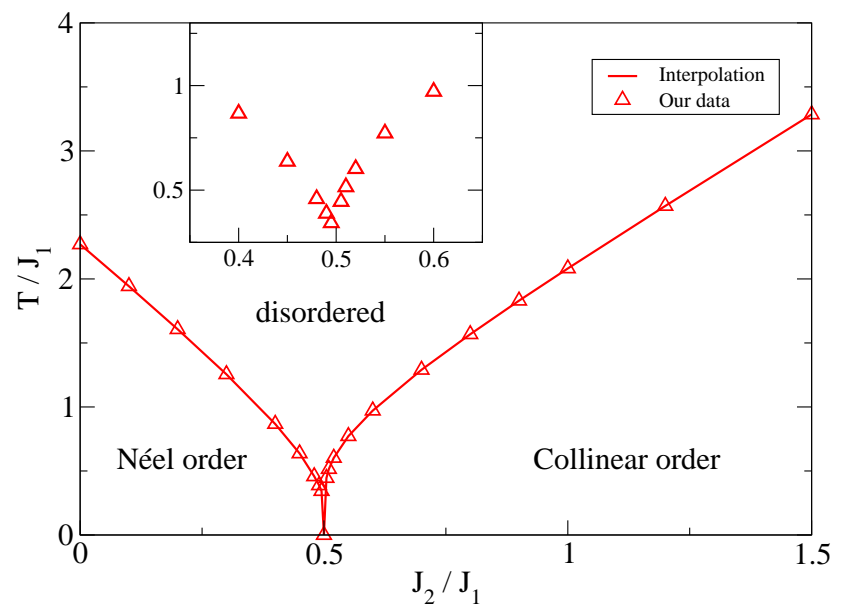

figure 2.2: (Published in [KHFP09].) Phase diagram for the isotropic frustrated $J_{1}-J_{2}$ Ising model. The phase transition on the left-hand side is continuous and Ising universal whereas the phase transition on the right-hand side is under debate and will be discussed in chapter 5 .

critical exponents [Ons44]. The set of Ising critical exponents define the two-dimensional Ising universality class [Gol93] and are also valid for many other phase transitions, for example, the static critical behavior of the gas-liquid transition. It was shown earlier [KHFP08, LB05] that for small $J_{2}<J_{1} / 2$ this universality holds also for the phase transition from the paramagnetic state into the Néel state for the frustrated system; however, the critical temperatures are reduced by the influence of the competing interactions, see figure 2.2 and [KHFP08, LB05].

The phase transition from the high-temperature phase into the collinear state (righthand side in figure 2.2) is one major subject of this thesis and will be discussed in detail in chapter 5; possible scenarios are either continuous transitions with Ising exponents, or varying exponents, or a weak first-order transition (see [KHFP08, LB05, MLAGS93] and the introduction of this thesis).

The direct transition from the Néel state to the collinear state is only possible at $T=0$ and is first order since the symmetries of the two configurations are different. This transition will be discussed in more detail for the quantum case because the degeneracy of the classical ground state may give rise to interesting phenomena for non-zero quantum fluctuations.

For the anisotropic case there are predictions that the phase transition to the SFAF phase is not direct, but there may exist an intermediate temperature regime where incommensurate ordering sets on before the system orders completely in the ground state, see [CG05].

\section{Ashkin-Teller model}

A very similar model is the Ashkin-Teller model. This lattice model was introduced as a generalization of the Ising model to a four-component system [AT43]. However, in the 1970s it was shown that the model can be mapped onto a system of two Ising models $(A$ and $B$ ) residing on the same lattice and interacting via an additional four-spin interaction [FW70]:

$$
H_{\mathrm{AT}}=J \sum_{\mathrm{NN} \in \mu=A, B} S_{i, \mu} S_{j, \mu}+J_{4} \sum_{\mathrm{NN}} S_{i, A} S_{j, A} S_{i, B} S_{j, B}
$$

The phase diagram is rather complicated and can be found, e.g., in [Bax82]. More important in the context of this thesis is the behavior of the phase transition from a 
high-temperature disordered state to magnetically ordered ground state: for a certain parameter region (in the $J$ vs. $J_{4}$ space) weak universal behavior is observed.

Furthermore, the two limiting cases of (1) the Ashkin-Teller model with $J_{4}=0$ and (2) the frustrated Ising model with $J_{1}=0$ are equivalent and represent two decoupled Ising models on sublattices $A$ and $B$.

\section{Heisenberg model $\mid 2.3$}

The above introduced Ising model is of purely classical nature and its finite spin variables are defined in one dimension and have only two possible states. The generalization of the spin with a fixed absolute value $S$ to a three-dimensional vector leads to the definition of the classical Heisenberg model:

$$
H_{\mathrm{HM}}=\sum_{i \neq j} \mathbf{J}_{i, j} \mathbf{S}_{i} \mathbf{S}_{j}
$$

where the exchange integral $\mathbf{J}_{i, j}$ has also become a vectorial quantity. For classical spins $\mathbf{S}_{i}$ all possible local states are now classified by a sphere of radius $S$ at site $i$. However, in this work the spins will be quantized, i.e., the value of $\mathbf{S}_{i}$ is quantized and the spin operators are defined by the commutation relations of $S_{i}^{x, y, z}$,

$$
\text { e.g. }\left[S_{i}^{x}, S_{j}^{y}\right]=i \delta_{i, j} S_{i}^{z} \quad \text { and } \quad\left[S_{i}^{n}, S_{j}^{n}\right]=0
$$

The basis of the Hamiltonian (2.15) is chosen to be in $z$ direction, thus, the quantization applies to the $S^{z}$ values of the spin operators. Only semi-integer values in the range of $\pm S$ are allowed. ${ }^{7}$ Throughout this whole thesis the local spins have a value of $S_{i}=\frac{1}{2}$ and hence $S_{i}^{z}= \pm \frac{1}{2}$. In a next step ladder operators can be introduced which increase/decrease the local magnetic moment $S_{i}^{z}$ by \pm 1 if possible and change the state accordingly:

$$
\begin{aligned}
& S_{i}^{+}=S_{i}^{x}+i S_{i}^{y}, \quad S_{i}^{-}=S_{i}^{x}-i S_{i}^{y} \quad \rightarrow \quad S_{i}^{x} S_{j}^{x}+S_{i}^{y} S_{j}^{y}=\frac{1}{2}\left(S_{i}^{+} S_{j}^{-}+S_{i}^{-} S_{j}^{+}\right) \\
& S^{+}|\downarrow\rangle=|\uparrow\rangle, \quad S^{-}|\uparrow\rangle=|\downarrow\rangle, \quad S_{1}^{+} S_{2}^{-}|\downarrow \uparrow\rangle=|\uparrow \downarrow\rangle, \quad S_{1}^{-} S_{2}^{+}|\uparrow \downarrow\rangle=|\downarrow \uparrow\rangle .
\end{aligned}
$$

The Hamiltonian (2.15) for $J^{x}=J^{y}=J^{x, y}$ then reads:

$$
H_{\mathrm{HM}}=\sum_{i \neq j} \frac{J_{i, j}^{x, y}}{2}\left(S_{i}^{+} S_{j}^{-}+S_{i}^{-} S_{j}^{+}\right)+J_{i, j}^{z} S_{i}^{z} S_{j}^{z} .
$$

Because all operators $S_{i}^{z}$ commute with each other and both the quantum spin$1 / 2$ Heisenberg model and the classical Ising models are locally two-state models, the anisotropic limit with $J^{x, y}=0$ of (2.15) is given exactly by an Ising model with $S_{i}= \pm \frac{1}{2}$ and $J^{z}=J$. For non-zero interactions $J^{x}=J^{y} \neq 0$ quantum fluctuations are introduced as shown above and change the physics of the limiting Ising case. This point of view will be the subject of the second part of this thesis: what happens if quantum fluctuations perturb the classical ground state of a frustrated Ising model - in particular in the vicinity of a highly frustrated point.

\footnotetext{
${ }^{7}$ Thus, for the quantum case the absolute value of $S$ is relevant for the physical properties as the number of states depends on $S$. In the limit of large $S$ the quantization of $S^{z}$ ceases to be important and the model resembles its classical limit.
} 
$J_{k}^{z}>0$ will be chosen antiferromagnetic as for the Ising case, but $J_{k}^{x, y}<0$ will be chosen ferromagnetic. In that way the frustration is lifted for the interactions in $x$ and $y$ directions which allows a simulation of the model using quantum Monte-Carlo techniques without a sign problem. ${ }^{8}$

\section{\begin{tabular}{l|l} 
hard-core bosons & 2.3 .1
\end{tabular}}

With the negative sign of the exchange interaction $J_{k}^{x, y}<0$ a mapping of the spin model onto a system of hard-core bosons [MM56] can be defined by the following operators:

$$
\begin{array}{ll} 
& {\left[b_{i}, b_{j}^{\dagger}\right]=i \delta_{i, j}, \quad n_{i}=b_{i}^{\dagger} b_{i}, \quad\left(b_{i}^{(\dagger)}\right)^{2}=0} \\
\text { mapping: } & S_{i}^{+} \rightarrow b_{i}^{\dagger}, \quad S_{i}^{-} \rightarrow b_{i}, \quad S_{i}^{z} \rightarrow n_{i}-\frac{1}{2} .
\end{array}
$$

The Hamiltonian (2.19) thus represents a model of bosons residing on the same geometric lattice as the spin model (with a bosonic exchange rate $t_{k}=J_{k}^{x, y} / 2$ ) with the restriction that every site can be occupied by one boson at most - hence the expression hard-core boson - that experience a repulsive interaction $\left(V_{k}=J_{k}^{z}\right)$ if two bosons occupy neighboring sites. The mapping also yields a constant term and terms for the chemical potential proportional to the density operators $n_{i, j}$ which scale with the interaction strengths $V_{k}$. At fixed half filling - the point of interest in this work - these terms only yield a constant irrelevant contribution. An additional magnetic field in the original spin Hamiltonian would change the filling of the bosonic model as it acts as an chemical potential, too.

\section{square lattice}

The definition of neighboring sites depends on the particular choice of the lattice and range of the interactions. In chapter 7 the quantum case of the above mentioned square lattice (sketched in figure 2.1) is investigated and nearest and next-nearest neighbor interactions are included:

$$
\begin{aligned}
H_{\square} & =\sum_{\mathrm{NN}} t_{1}\left(b_{i}^{\dagger} b_{j}+b_{i} b_{j}^{\dagger}\right)+V_{1} n_{i} n_{j} \\
& +\sum_{\mathrm{NNN}} t_{2}\left(b_{i}^{\dagger} b_{j}+b_{i} b_{j}^{\dagger}\right)+V_{2} n_{i} n_{j} .
\end{aligned}
$$

\section{honeycomb lattice}

A very similar model is analyzed in chapter 8 for the honeycomb lattice. For this bipartite Bravais lattice with a two-site basis, quantum fluctuations are even more important because the number of nearest neighbors - the coordination number of the lattice is $n=3$ - is smaller than for the square lattice and is smallest for a two-dimensional lattice. How-

\footnotetext{
${ }^{8}$ This particular point will be explained in section 3.2.2.
} 
ever, here additional third-nearest neighbor (NNNN) interactions are taken into account motivated by earlier works on similar models (see introduction 1.2):

$$
\begin{aligned}
H_{\square} & =\sum_{\mathrm{NN}} t_{1}\left(b_{i}^{\dagger} b_{j}+b_{i} b_{j}^{\dagger}\right)+V_{1} n_{i} n_{j} \\
& +\sum_{\mathrm{NNN}} t_{2}\left(b_{i}^{\dagger} b_{j}+b_{i} b_{j}^{\dagger}\right)+V_{2} n_{i} n_{j} \\
& +\sum_{\mathrm{NNNN}} t_{3}\left(b_{i}^{\dagger} b_{j}+b_{i} b_{j}^{\dagger}\right)+V_{3} n_{i} n_{j} .
\end{aligned}
$$

The interactions between next-nearest and third-nearest neighbors is set to the same value $V_{2}=V_{3}$ and all fluctuations scale with a ratio of $t=t_{k} / V_{k}$.

In this thesis the focus will be on the spin interpretation of the models. However, for convenience and to emphasize the anisotropy of the models the parameters $t_{k}$ and $V_{k}$ will be employed:

$$
H_{\mathrm{HM}}=\sum_{k, i, j}\left[t_{k}\left(S_{i}^{+} S_{j}^{-}+S_{i}^{-} S_{j}^{+}\right)+V_{k}^{z} S_{i}^{z} S_{j}^{z}\right]=\sum_{k, i, j}\left[2 t_{k}\left(S_{i}^{x} S_{j}^{x}+S_{i}^{y} S_{j}^{y}\right)+V_{k}^{z} S_{i}^{z} S_{j}^{z}\right],
$$

whereby the index $k$ represents the range of the interactions.

$$
\begin{array}{l|l}
\text { ground states } & 2.3 .2
\end{array}
$$

In quantum mechanics not only thermal but also quantum fluctuations may suppress the ordering of the system into a long-range ordered ground state. This mechanism is even more important in low-dimensional systems. For isotropic quantum models in one and two dimensions that inhabit a continuous symmetry - in the isotropic Heisenberg model this symmetry is given by spin rotational invariance - the Mermin-Wagner theorem states that no long-range order is stabilized for non-zero temperatures, see [Hoh67, MW66].

In the present model the interaction is chosen to be strongly anisotropic and, hence, the Mermin-Wagner theorem does not apply. Thus, starting from the classical limit of zero quantum fluctuations a classical magnetic long-range order - the Néel and collinear configuration - may also be stabilized in the quantum system, even at finite temperatures. Even if the classical states are no exact eigen-states of the quantum Hamiltonian the order parameter can be finite if long-range correlations are stable.

For the square lattice the antiferromagnetic configurations are exactly as shown in figure 2.1 and the critical point is also the same; However, the energies in (2.10) are scaled by a factor $S^{2}=\frac{1}{4}$ which will be important for the perturbational approach presented in section 7.1. The static structure factor serves as order parameter for the same momentum vectors too.

For the honeycomb lattice the classical ground states are shown in figure 2.3. In the Néel state all spins of the same sublattice $A$ are aligned parallel and anti-parallel to the spins of the sublattice $B$, hence the ordering wave vector is given by $\mathbf{q}=(0,0)$. The collinear state favored by the nearest-neighbor interactions - also minimizing the third-nearest neighbor interactions of the same strength $\left(V_{2}=V_{3}\right)$ - is given by a parallel alignment of all spins inside a row and anti-parallel alignment for the perpendicular row of spins. The state is sixfold degenerate and at three wave vectors the structure factor gives 


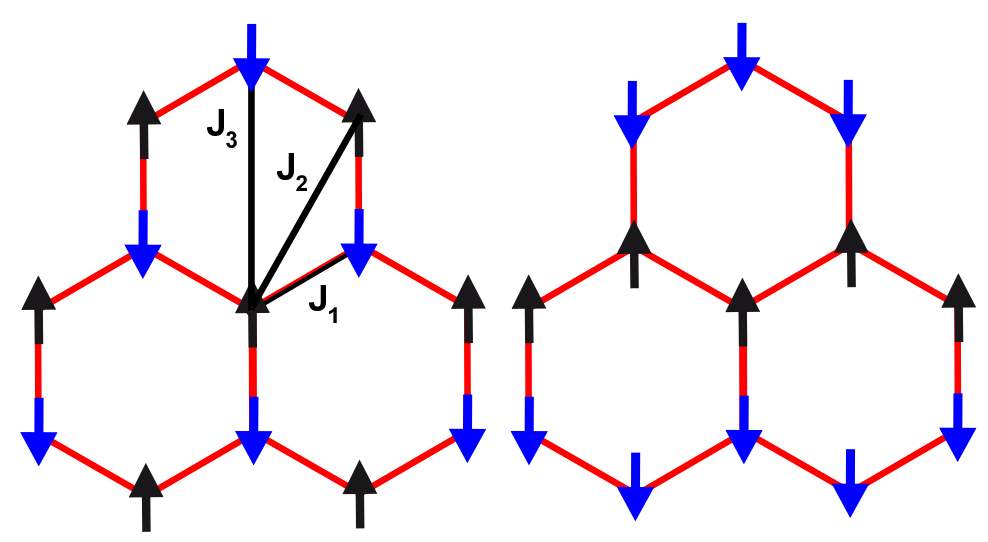

(a) Néel state with all spin interactions $J_{k}$ (b) collinear state with $M_{\square}=0$

figure 2.3: Classical antiferromagnetic ground states of the honeycomb lattice for different strengths of frustration - $J_{k}$ connecting nearest, next-nearest and third-nearest neighbors. (a) Néel state with spins in one sublattice aligned parallel and antiparallel to the other sublattice. (b) collinear state with parallel aligned spins along one direction and anti-parallel spins along the perpendicular direction.

a finite signal: $\mathbf{q}=\frac{\pi}{\sqrt{3}}(\sqrt{3}, 1)$ and $\mathbf{q}=\frac{\pi}{\sqrt{3}}(\sqrt{3},-1)$ with sublattices $A$ and $B$ parallel, $\mathbf{q}=\frac{2 \pi}{\sqrt{3}}(0,1)$ with $A$ and $B$ anti-parallel. The total magnetization of each hexagon holds $M_{\square}=\sum_{i \in \square} S_{i}^{z}=0$. The energies of the classical limit for a lattice of $N=2 \times L \times L$ sites are:

$$
\begin{aligned}
E_{\text {Néel }} & =-\frac{1}{8} N\left(3 V_{1}-6 V_{2}+3 V_{3}\right) \stackrel{V_{2}=V_{3}}{=}-\frac{3}{8} N\left(V_{1}-V_{2}\right), \quad \text { and } \\
E_{\text {coll }} & =\frac{1}{8} N\left(V_{1}-2 V_{2}-3 V_{3}\right) \stackrel{V_{2} \equiv V_{3}}{=}-\frac{1}{8} N\left(5 V_{2}-V_{1}\right)
\end{aligned}
$$

The transition point is thus at $V_{3}=V_{2}=V_{1} / 2$. This is the same value as for the square lattice and similarly the ground state is largely degenerate at the critical point. A collinear state with non-zero magnetization per hexagon may yield a lower energy for models with $V_{2} \neq V_{3}$ although the total magnetization will still be zero [MB12].

For both lattices, away from the critical point $\left(V_{2} \neq V_{1} / 2\right)$ the antiferromagnetic states are expected to be stable against small quantum fluctuations $t_{k} \neq 0$. A perturbational estimation for the stability of the two phases is presented in sections 7.1 and 8.1 for the square and honeycomb lattice, respectively.

For large fluctuations, which correspond to large ferromagnetic spin interactions in $x$ and $y$ direction in (2.15), a ferromagnetic order in the $x y$ plane is expected. The equivalent bosonic model is then dominated by the kinetic energy of the bosons and the repulsive interactions are negligible. However, due to the hard-core constraint the movement of the

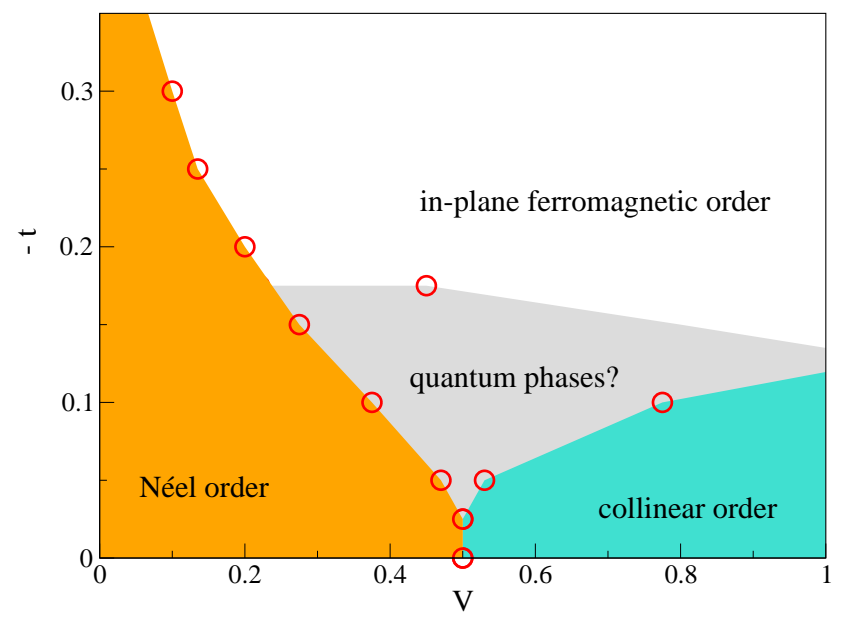

figure 2.4: (Published in $\left[\mathrm{KAC}^{+} 12\right]$.) Schematic phase diagram for the frustrated anisotropic Heisenberg model on the honeycomb lattice. The axes represent the frustration $V=V_{2} / V_{1}=V_{3} / V_{1}$ and the relative amplitude of the quantum fluctuations $t=t_{r} / V_{r}$. Red circles refer to actual transition points calculated by quantum Monte-Carlo simulations (see chapter 8). 
bosons has to be correlated and the ground state is a superfluid phase. The relation of ferromagnetic and superfluid order can be derived from the condensation of the bosonic excitations - magnon modes - of the magnetic model when it undergoes the transition into the ferromagnetic ground state, see [Blo32, Hoe50, MM56]. An upper bound for the energy of this state can be obtained by assuming a product state of spins aligned in the $x$ (or $y$ ) direction and rewriting the Hamiltonian in (2.24) in the basis of $S^{x}$ as

$$
H_{\mathrm{HM}}=\sum_{k, i, j}\left[2 t_{k} S_{i}^{x} S_{j}^{x}+\frac{2 t_{k}+V_{k}}{4}\left(S_{i}^{+} S_{j}^{-}+S_{i}^{-} S_{j}^{+}\right)+\frac{2 t_{k}-V_{k}}{4}\left(S_{i}^{+} S_{j}^{+}+S_{i}^{-} S_{j}^{-}\right)\right] .
$$

In the described product state the energies only depend on the $S^{x}$ interaction due to the rotational invariance of the ferromagnetic state:

$$
E_{\text {ferro, } \square}=N\left(t_{1}+t_{2}\right) \quad \text { and } \quad E_{\text {ferro, } \square}=\frac{3}{4} N\left(t_{1}+2 t_{2}+t_{3}\right) \stackrel{t_{2}=t_{3}}{=} \frac{3}{4} N\left(t_{1}+3 t_{2}\right) .
$$

The limit of zero $S^{z}$ interactions yields the $x y$ model which is known to exhibit a finite magnetization in the $x y$ plane [Jia11, SH99].

A superposition of antiferromagnetic $S^{z}$ order and ferromagnetic in-plane order is referred to as a supersolid state, which stems from the bosonic description where superfluid order and a boson-density wave are established at the same time. This kind of intermediate configuration manifests itself by showing finite signals for multiple order parameters and has been discussed in earlier works for similar models [CMWK08, HD05, LF73, MPB ${ }^{+}$05, NC08, WT05] where a stable ground state was verified away from half-filling.

A possible ground-state phase diagram is sketched in figure 2.4; the red circles represent quantum Monte-Carlo data for the frustrated anisotropic model on the honeycomb lattice but the sketch applies for both systems. The most interesting case for the quantum models is the intermediate regime of high frustration $\left(J_{2} \approx J_{1} / 2\right)$ and small but finite quantum fluctuations. As was outlined in the introduction the appearance of quantum ordered phases is most probable for these parameters.

\section{quantum phases}

The building blocks of these phases are not single spins but rather entangled states of multiple spins; the smallest and most discussed unit is a dimer consisting of two spins with total $\operatorname{spin} S_{\text {total }}^{z}=0$

$$
\begin{aligned}
& \left|S_{0}\right\rangle=\left|S^{\text {total }}=0, S^{z}=0\right\rangle=\frac{1}{\sqrt{2}}(|\uparrow \downarrow\rangle-|\downarrow \uparrow\rangle) \quad \text { or } \\
& \left|S_{1}\right\rangle=\left|S^{\text {total }}=1, S^{z}=0\right\rangle=\frac{1}{\sqrt{2}}(|\uparrow \downarrow\rangle+|\downarrow \uparrow\rangle)
\end{aligned}
$$

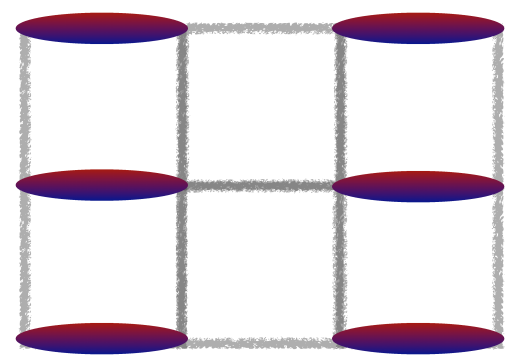

(a) columnar dimer state

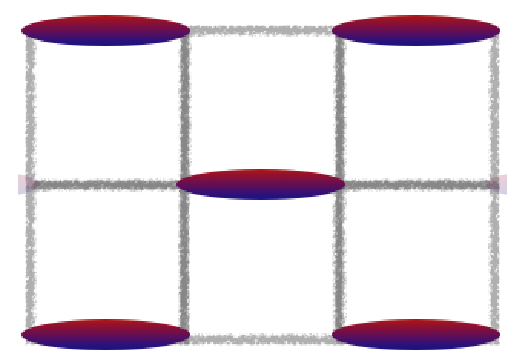

(b) staggered dimer state

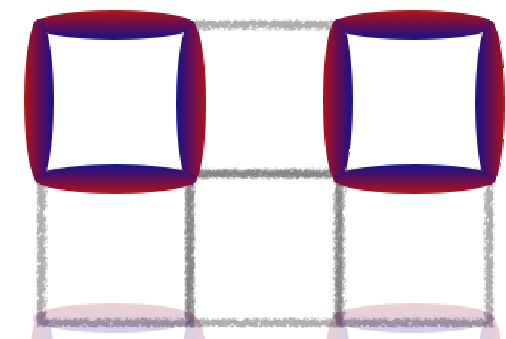

(c) plaquette state

figure 2.5: Three different quantum phases with a long-range ordered covering of the lattice with superpositions of multiple spins represented by the colored shapes. 
which minimizes not only its potential but also its kinetic energy:

$$
\begin{aligned}
& {\left[J^{x, y} / 2\left(S_{1}^{+} S_{2}^{-}+S_{1}^{-} S_{2}^{+}\right)+J^{z} S_{1}^{z} S_{2}^{z}\right]\left|S_{0}\right\rangle=\left(-J^{x, y} / 2-J^{z} / 4\right)\left|S_{0}\right\rangle} \\
& {\left[J^{x, y} / 2\left(S_{1}^{+} S_{2}^{-}+S_{1}^{-} S_{2}^{+}\right)+J^{z} S_{1}^{z} S_{2}^{z}\right]\left|S_{1}\right\rangle=\left(J^{x, y} / 2-J^{z} / 4\right)\left|S_{1}\right\rangle .}
\end{aligned}
$$

The covering of the whole lattice with these dimers allows for different phases such as the columnar or staggered dimer phase sketched in figure 2.5 or phases with larger periods of long-range ordered configurations. Furthermore, larger building blocks as plaquettes of four spins are possible (figure 2.5c). The long-range order of these phases breaks a symmetry of the Hamiltonian and can be detected by calculating higher order correlation functions. However, another possibility is a dimer covering without long-range order - the so-called spin-liquid state. The covering of the lattice is not given by a static configuration of dimers but represents a superposition of all possible dimer coverings. The state is fully symmetric and cannot be detected by any local order parameter [And73, Bal10]. Thus, a verification is only possible by excluding any kind of long-range order. There are two types of spin liquids - the algebraic and gapped form [Bal10] - that show different excitations. The excitations for the algebraic form in two dimensions are of particular interest because their distribution function can reflect fermionic or bosonic character, or alternatively neither of them [Kit06]. The test for a topological ordering in such a disordered state can be accomplished by calculating these low-lying excitations. For a periodic system (with an aspect ratio close to one) which exhibits topological order a ground-state degeneracy is expected [MLMS02]. 


\section{computational methods $\mid 3$}

summary

The specific methods used throughout this work will be described: to solve the models given in section 2.3 numerically one can diagonalize the matrix representation of the quantum mechanical Hamilton operator for small system sizes. This method - scaling exponentially in the system size $N$ - and some options how to extend the accessible lattice size (up to $N=36$ sites) are described in section 3.1. In order to analyze larger systems the concept of Monte-Carlo simulations is introduced for the statistical evaluation of the partition function and calculation of physical observables as energy and order parameters. The Monte-Carlo simulations scale in powers of $N$, and the exact order of the scaling also depends on the observables that are computed. The classical implementation for the Ising model with up to four million sites will be the subject of section 3.2.1. For the quantum model a more sophisticated and time-consuming approach is necessary that will be introduced and explained in section 3.2.2. A numerical calculation of the free energy using transfer-matrices is presented at the end of the chapter.

\section{\begin{tabular}{l|l} 
exact diagonalization & 3.1
\end{tabular}}

Because the Hamilton operator of a quantum mechanical system is in general not diagonal in a common basis like the $S^{z}$ basis, the Hamiltonian has to be diagonalized to obtain the eigen-energies and states of the model. The transformation of a Hamiltonian in the thermodynamic limit into its diagonal form using only analytic expressions is only possible for very special systems. For all other models this task has to be performed numerically 
and is thus limited to finite systems. As a short example, the transformation of the singlet and triplet state for the anisotropic Heisenberg coupling of two spins is shown:

$$
\begin{aligned}
H & =\frac{J^{x, y}}{2}\left(S_{1}^{+} S_{2}^{-}+S_{1}^{-} S_{2}^{+}\right)+J^{z} S_{1}^{z} S_{2}^{z} \\
& =\left(\begin{array}{cccc}
-\frac{J^{z}}{4} & \frac{J^{x, y}}{2} & 0 & 0 \\
\frac{J^{x, y}}{2} & -\frac{J^{z}}{4} & 0 & 0 \\
0 & 0 & \frac{J^{z}}{4} & 0 \\
0 & 0 & 0 & \frac{J^{z}}{4}
\end{array}\right) \text { basis: } \begin{array}{l}
|\uparrow \downarrow\rangle \\
|\uparrow \uparrow\rangle
\end{array} \\
\underset{\operatorname{diagonalize}}{\longrightarrow}\left(\begin{array}{cccc}
-\frac{J^{x, y}}{2}-\frac{J^{z}}{4} & 0 & 0 & 0 \\
0 & \frac{J^{x, y}}{2}-\frac{J^{z}}{4} & 0 & 0 \\
0 & 0 & \frac{J^{z}}{4} & 0 \\
0 & 0 & 0 & \frac{J^{z}}{4}
\end{array}\right) \text { basis: } & \frac{1}{\sqrt{2}}(|\uparrow \downarrow\rangle+|\downarrow \uparrow\rangle)
\end{aligned}
$$

This transformation becomes more complicated for two-dimensional lattices and longrange interactions due to the increasing number of non-zero entries in the matrix. The dimension of the matrix is given by the total number of states $m=q^{N}$ where $N$ is number of sites and $q$ is the number of states per site. In the particular case of a spin- $1 / 2$ model holds $q=2$.

$$
\begin{array}{l|l}
\text { symmetries } & 3.1 .1
\end{array}
$$

The computational effort to diagonalize matrices does not scale linearly with dimension $m$ but in higher powers [GvL96]. The details of different algorithms will be discussed in the next subsection. However, for all cases it is useful to split the matrix into smaller parts which can be diagonalized more efficiently, i.e., a block-diagonal form is constructed. This splitting is directly connected with the symmetries of the Hamilton operator, i.e., only basis states that are connected by off-diagonal matrix elements have to be diagonalized simultaneously.

Every symmetry is connected with an operator $A$ commuting with the Hamiltonian; the most obvious conserved symmetry inside the Heisenberg model is the conservation of the total spin $A=\sum_{i} S_{i}^{z}$, i.e., the commutator holds

$$
\sum_{i}\left[H_{\mathrm{HM}}, S_{i}^{z}\right]=0
$$

The Hamiltonian for a spin- $1 / 2$ system is split into $N+1$ subspaces which have a dimension $\left(\begin{array}{l}N \\ k\end{array}\right)$ where $k=\frac{N}{2}-S_{\text {total }}^{z}$. To bring the Hamiltonian into the corresponding block diagonal form a simple rearrangement of the basis states may be necessary but no basis transformation is needed. For most cases throughout the present work the subspace $S_{\text {total }}^{z}=0$ is the most interesting since the dominating interactions will be chosen antiferromagnetic. However, this subspace is the largest and needs further divisions to allow for a diagonalization of larger systems. The implementation of periodic boundary conditions yields a further symmetry in momentum space, i.e., the model is invariant under certain translations. To use this symmetry, the basis has to be transformed by applying a Fourier transformation, for more details see [Sch03, GvL96]. Other symmetries depending 
on the momentum subspace may be conserved and can yield further simplifications, as for example, inversion or rotation symmetries. The remaining smaller subspaces can be diagonalized by different algorithms.

\section{\begin{tabular}{l|l} 
algorithms & 3.1 .2
\end{tabular}}

A full diagonalization of the Hamilton operator for a spin-1/2 Heisenberg model is only possible for $N \leq 24$ (the accessible size depends on the symmetries of the model as described above). For this purpose the Householder algorithm is used that is based on orthogonal matrix transformations and scales with the third power of the matrix dimension $\mathrm{m}^{3}$ independently on the number of empty entries in the matrix [GvL96]. The result is a complete spectrum and the corresponding set of eigen-vectors.

For larger systems - keeping in mind the exponential scaling of $m$ with $N$ - the goal of the diagonalization has to be chosen less ambitiously. In general this is in agreement with the interest in the ground-state behavior of the models. The most common approach to calculate the lowest eigen-values of a large sparse ${ }^{1}$ matrix is the Lanczos algorithm. Within the Lanczos algorithm an effective tridiagonal Hamiltonian is constructed that is based on a Krylov space [GvL96, Par98] and has a significantly smaller dimension than the original Hamiltonian. It can be shown that the lowest eigen-values and related eigen-vectors of this effective Hamiltonian are very good approximations of the lowest eigen-values and eigen-vectors of the original Hamiltonian [GvL96, Par98]. This effective Hamiltonian can be set up efficiently by applying a matrix-vector multiplication recursively and, therefore, allows for larger system sizes than accessible within a complete exact diagonalization. The more entries of the original matrix that are non-zero the slower the convergence of the algorithm. Thus, by applying the method to the models given in section 2.3 the accessible system size for the honeycomb lattice will be smaller than for the square lattice, because in the square lattice only four bonds per site contribute to the Hamiltonian where on the honeycomb lattice twelve bonds are considered.

The program for the full diagonalization and the Lanczos method that is used to calculate the lowest eigen-values of the anisotropic Heisenberg model for some parameters was developed by Jörg Schulenburg within his PhD thesis [Sch, Sch03].

\section{\begin{tabular}{l|l} 
Monte-Carlo simulations & 3.2
\end{tabular}}

In section 2.1 it was stated that the thermodynamic properties of a model can be deduced from the canonical partition function. This partition function is not available as a closed analytic expression for the two-dimensional models discussed in the previous chapter. A well-established method to approximately calculate integrals or large sums is the MonteCarlo algorithm. The essential idea of the algorithm is based on the stochastic evaluation of random events. ${ }^{2}$

In the context of condensed matter theory the algorithm is used to sample the configuration space of a model and to calculate observables in the sampled configurations, thus, a

\footnotetext{
${ }^{1} \mathrm{~A}$ sparse matrix is considered to contain mostly entries that equal zero which is given for models that couple only a few neighboring sites.

${ }^{2}$ The name stems from an urban district in the principality of Monaco which is widely known for its large casino.
} 
measurement is simulated. The sampling itself is a Markov process and can be performed in different ways according to the underlying problem or observables of interest [Ber04]. The importance sampling is based on choosing different configurations in phase space by a weighting function and evaluating observables in these configurations statistically. Two crucial requirements should be fulfilled by an implementation of an importance sampling Monte-Carlo simulation according to the master equation of the sampled process: ergodicity and detailed balance [Ber04, LB05].

\section{\begin{tabular}{l|l} 
classical Monte-Carlo algorithm & 3.2 .1
\end{tabular}}

The algorithm proposed by Metropolis et al. in reference $\left[\mathrm{MRR}^{+} 53\right]$ meets the required standards of ergodicity and detailed balance, and is used for the classical simulations in chapters 5 and 6 . The transition is divided into two steps: selecting a new state and accepting or rejecting it. The probability to select a new state is the same for all starting and end points, i.e., new states will be proposed following a uniform distribution. However, the acceptance rate is directly coupled to the weight of both states inside the canonical ensemble, i.e., the transition probability $u(i, j)$ is connected to the Boltzmann weight of the energy difference $p_{i, j}=\exp \left(-\beta\left(E_{j}-E_{i}\right)\right)$ of both states $i$ and $j$. Per definition the probability holds $0 \leq u(i, j) \leq 1$ and thus for negative energy difference every new state is accepted:

$$
u(i \rightarrow j)=\min \left\{1, \exp \left(-\beta\left(E_{j}-E_{i}\right)\right)\right\}
$$

For the Ising model a single-spin flip of the Metropolis algorithm is performed as such:

1. select a lattice site, either randomly or systematically, and propose to flip the spin located at the site

(2.) calculate the Boltzmann weight $p_{i, j}$ for the energy difference

(3.) generate a random number $0<r \leq 1$ using a uniform distribution

(4.) if $r<p_{i, j}$ accept the spin flip and change the configuration accordingly, otherwise reject the change

5. calculate observables (measurement)

Steps $2-4$ are only necessary if the energy of the proposed new state is higher than the original energy.

A Monte-Carlo simulation is performed in units of sweeps ( $N$ single spin flips) and observables should only be calculated when the overall configuration has changed sufficiently, i.e., the correlation between subsequent measurements of an observable should be minimized [Ber04]. In addition, each simulation needs to thermalize in the beginning from its initial (random) state into a state of equilibrium according to the fixed temperature, i.e., after a reasonable number of sweeps only small fluctuations of the energy are expected, see also [LB05]. 


\section{improvements}

In the vicinity of a critical temperature the configuration of the system undergoes drastic changes. The energy and other observables show large fluctuations and hence the singlespin update may suffer critical slowing down since the proposed changes are very small [LB05]. In addition to this usual problem, because of the degeneracy at the critical point of the discussed models (see subsection 2.2.1) the free-energy landscape has many local minima for parameters close to the critical point. As a consequence, it is very complicated to reach the correct equilibrium state without further enhancements to the algorithm.

Possible improvements depend on the model and the nature of the critical point. A typical approach is cluster updates [SW87, Wol89], but these are not suitable for the antiferromagnetic Ising model with long-range interactions as the clusters cover the complete lattice quickly. However, because the ground-state degeneracy of these models can be tracked down by flipping complete lines of spins (see subsection 2.2.1), the update of such a predefined cluster can be used to improve the convergence of the algorithm in the vicinity of the degenerate point.

A second possible improvement is an exchange of the complete configuration - called exchange Monte-Carlo or parallel tempering. Here a set of simulations at different temperatures are computed simultaneously and swaps between these configurations are proposed, see [Han97, HN96, KTHT06]. The acceptance probability of such a swap once again has to fulfill the detailed balance:

$$
p_{\text {swap }}=\exp (-\Delta \beta \cdot \Delta E), \quad \Delta \beta=\beta_{i}-\beta_{j} \text { and } \Delta E=E_{i}-E_{j} .
$$

This procedure enhances the thermalization process drastically, in particular in the vicinity of critical points which exhibit a large degeneracy. The degeneracy allows for many configurations with similar energies (representing local minima) which can be exchanged rapidly by the parallel-tempering procedure to saturate in the configuration with the global minimal energy.

More details on the implementation of the classical Monte-Carlo algorithm can be found in appendix A.1.

\section{\begin{tabular}{l|l} 
quantum Monte-Carlo algorithm & 3.2 .2
\end{tabular}}

The representation and simulation of quantum mechanical models is more cumbersome than the classical Monte-Carlo. The reason for this are the non-commuting operators that contribute to the quantum Hamiltonian and hamper the decomposition of the partition function.

An idea by Handscomb for an expansion of the partition function [Han62] was the basis for the development of an algorithm by Sandvik which is known as directed loop stochastic series expansion [San92, SK91, SS02]. The subsequent outline of the method is following the description given in reference [SS02].

Since the partition function is a sum over exponential functions a reasonable ansatz is simply expanding these functions:

$$
\begin{aligned}
Z_{\text {can. }} & =\operatorname{Tr}[\exp (-\beta H)]=\sum_{n} \frac{\beta^{n}}{n !} \operatorname{Tr}\left[(-H)^{n}\right] \\
& =\sum_{\alpha} \sum_{n} \frac{\beta^{n}}{n !}\left\langle\alpha\left|(-H)^{n}\right| \alpha\right\rangle
\end{aligned}
$$


where $|\alpha\rangle$ represents a complete set of basis vectors for the system. For the anisotropic spin-1/2 Heisenberg models, which will be the subject of investigation in chapters 7 and 8 of this work, a suitable and accessible basis is given by the $S_{i}^{z}$ product representation. The Hamiltonian is then split up into diagonal parts $H^{\text {dia }}$, i.e., $S^{z}$ operators, and off-diagonal parts $H^{\text {off }}$ that change the $S^{z}$ product state. Furthermore, the sum is divided up into operators $H_{b_{i}}$ which only act upon a single bond $b_{i}$ of the lattice:

$$
Z_{\text {can. }}=\sum_{\alpha} \sum_{n} \sum_{S_{n}} \frac{\beta^{n}}{n !}\left\langle\alpha\left|\prod_{i=1}^{n}\left(-H_{b_{i}}\right)\right| \alpha\right\rangle .
$$

The index $S_{n}$ represents a series of indices $b_{i}$, also called operator-index sequence, which introduces an additional dimension, since the state of the quantum spin system is no longer described by a single configuration $|\alpha\rangle$ but requires this supplemental sequence of operators acting upon $|\alpha\rangle$. The initial state $|\alpha(0)\rangle$ is evolved by applying a product of operators $H_{b_{i}}$

$$
|\alpha(p)\rangle \propto \prod_{i=1}^{p} H_{b_{i}}|\alpha(0)\rangle .
$$

weights and sign problem

The sign in front of the off-diagonal Hamiltonian segments $H_{b_{i}}^{\text {off }}$ in equation (3.7) plays a crucial role. The sampling of this representation of the partition function using MonteCarlo update schemes is only possible in principle if all weights

$$
p_{\alpha, S_{n}}=\frac{\beta^{n}}{n !}\left\langle\alpha\left|\prod_{i=1}^{n}\left(-H_{b_{i}}\right)\right| \alpha\right\rangle
$$

are positive. Hence, for positive exchange rates in the off-diagonal parts $H^{\text {off }}$ an overall negative sign may remain ans this hampers the application of stochastic evaluations. For a bipartite lattice - such as the square or honeycomb lattices - the additional condition of periodicity in the evolution of the states $|\alpha(n)\rangle=|\alpha(0)\rangle$ guarantees that an even number of off-diagonal operators appears in the operator sequence. However, because of the frustration in the models presented in chapter 2 this guarantee does not hold, and the exchange rates for the operators acting upon bonds which connect sites of the same sublattice have to be chosen to be negative to avoid the sign problem completely. ${ }^{3}$ The sign for operators connecting sites of different sublattices can be mapped onto each other via a sublattice rotation, and can therefore be chosen freely.

\section{directed loop update}

The expansion order $n$ of the partition function can be linked directly to the internal energy of the system using equations (2.3) and (3.6):

$$
E=-\frac{1}{Z} \frac{\partial Z}{\partial \beta}=-\left\langle\frac{n}{\beta}\right\rangle .
$$

\footnotetext{
${ }^{3}$ It is also possible to apply Monte-Carlo techniques if the overall sign is negative by taking all weights positive and keeping track of a so-called average sign. However, in most cases this average sign decreases exponentially with the system size and inverse temperature $\beta$. Thus, an evaluation including the reciprocal average sign is hampered by poor statistics. This effect is usually referred to as the true sign problem for quantum Monte-Carlo simulations [Wie93].
} 
Because the finite system has a negative upper bound for the internal energy, the expansion order has a positive upper bound and the expanded version of $Z_{\text {can. }}$ allows for a statistically exact representation. This detail is very useful for the practical implementation of the Monte-Carlo update and also shows that the expansion order grows with the inverse temperature $\beta$.

As mentioned above, the basis for such an implementation is a starting configuration in the $S_{i}^{z}$ basis for the spin system and a sequence of operators acting upon that state. The length of this sequence is given by a fixed number $m \geq n$ which can be increased if necessary. All operators are initially set to identity $\mathbb{1}$. The update consists of two steps.

1. The Monte-Carlo step regulates according to a weight function [SS02] - obeying detailed balance - the number of non-identity operators in the sequence $S_{m}$ by exchanging local diagonal operators and identity operators.

2. In a second step a directed closed loop is built inside the operator sequence which connects non-identity operators in a determined manner. The update of this loop interchanges per definition diagonal and off-diagonal operators and flips the connected spins.

The setup of the directed loop is the crucial part of the Monte-Carlo simulation. For each addressed vertex describing a local operator and the adjacent four spins (all operators represent two-spin interactions, hence, two spins come in and two come out) the exit leg has to be calculated regarded to the incoming leg. The procedure stops when the loop reaches the starting leg of the initial vertex. The periodicity in the operator sequence is thus necessary.

In this work an implementation of this algorithm by Alet et al. [AWT05] is used which is embedded in the ALPS project $\left[\mathrm{AAC}^{+} 07, \mathrm{ADG}^{+} 05, \mathrm{BCE}^{+} 11\right]$. For more details of the implementation see appendix A.2.

\section{improvements}

The efficiency of the loop update can be enhanced by imposing further requirements on the setup of the loop, e.g., the conservation of the total spin, and the prohibition of selecting the incoming leg as exit leg to decrease the probability of short loops. Thus, a faster thermalization can be reached.

Concerning the thermalization problems at phase transitions, the quantum fluctuations introduce a kinetic energy in the system which helps to overcome small energy barriers and. However, because the quantum models are frustrated and the additional kinetic energy also reduces the transition temperatures, further improvements of the algorithm are necessary. Once again an exchange Monte-Carlo helps to reduce the thermalization time of low-temperature simulations significantly. For the stochastic series expansion an exchange of different configurations $i$ and $j$ at different temperatures is connected with the expansion order $n$ [SSC02, Mel07]:

$$
p_{\text {swap }}=\min \left\{1,\left(\frac{\beta_{i}}{\beta_{j}}\right)^{n_{j}-n_{i}}\right\} .
$$

The interesting physics for the quantum models happens at lower temperatures than for the classical models, and hence the inverse temperatures increase very quickly. To ensure a proper acceptance rate even at low temperatures a dense set of configurations is thus 
necessary. The drawback of this method is that the simulation time for all temperatures is dictated by the highest value of $\beta$, since the expansion order $n$ and the simulation time grow with $\beta$. Therefore, in non-frustrated systems a single simulation that reaches the desired temperature stepwise, i.e., in an annealing procedure, may be sufficient. This approach is also useful for the present case to prethermalize the simulations before the time-consuming exchange Monte-Carlo algorithm is applied.

\section{\begin{tabular}{l|l} 
observables & 3.2 .3
\end{tabular}}

In this work the focus is on static measurements, i.e., observables that can be evaluated directly in the configurations that are accessed by the Monte-Carlo simulation.

For classical simulations, energies and corresponding histograms and higher moments, as well as magnetic observables like the structure factor and correlation functions (described in section 2.1) can be calculated directly in the sampled states that are given in the $S^{z}$ basis. The expectation value of an observable $A$ is calculated as an average of consecutive measurements in the configurations $i$ as

$$
\langle A\rangle=\frac{1}{N} \sum_{i}^{N} A_{i} .
$$

This also holds for quantum Monte-Carlo simulations. However, here the statistics can be improved further not only by measuring in the initial state but also in the configurations evolved by the operator sequence. Since the differences between these configurations are very small, a technical optimization is introduced by gathering these measurements separately so that the contribution counts as a single value in the time series.

A more complicated task is the determination of ordering processes that give no signal in the $S^{z}$ basis. For the models discussed in section 2.3 a ferromagnetic ordering is expected for large quantum fluctuations in the $x y$ plane of the spin representation. Such a phase corresponds to a superfluid condensation of the equivalent hard-core bosons. Thus, a correlated movement of these bosons is expected and this can be detected by measuring the average direction of the bosonic hopping on the lattice. However, in the quantum Monte-Carlo simulation the loop updates and the outcoming operator sequence should be independent from each other and, hence, it is necessary to measure the absolute or squared value of the overall direction of off-diagonal operators in the operator sequence. Therefore, two counters $n_{x, y}$ are implemented (for a two-dimensional system) and are increased or decreased according to the direction of every off-diagonal operator in $S_{n}$, e.g.:

$$
S_{i}^{+} S_{j}^{-}=b_{i}^{\dagger} b_{j}: n_{x}^{\prime}=n_{x}+\left(i_{x}-j_{x}\right) \text { and } n_{y}^{\prime}=n_{y}+\left(i_{y}-j_{y}\right) .
$$

The superfluid density is then given by the normalized combination of these two values [PC87]:4

$$
\rho_{S}=\frac{n_{x}^{2}+n_{y}^{2}}{2 N \beta} .
$$

\footnotetext{
${ }^{4}$ It is also often referred to as winding number which stems from the introduction of this quantity in the context of liquid helium in a cylinder.
} 
For a spin system $\rho_{S}$ represents the spin stiffness with respect to $S^{z}$; it is not a direct order parameter for a ferromagnetic alignment of the spins in the $x y$ plane but provides clear evidence for a correlated configuration in the plane.

The ordered quantum phases discussed in section 2.3 consist of correlated dimers arranged in a long-range ordered configuration. To detect such a configuration it is necessary to estimate the correlation of more than two spins. Because the dimers on some bonds - in particular the bonds upon which the Hamiltonian acts directly - gain their energy from local diagonal and off-diagonal operations, it is possible to estimate the correlation of such dimers by analyzing the operator sequence. More precisely a direct sub-sequence of two operators inside the operator string $S_{n}$ indicates a correlation between these operations. Thus, a counter for every possible correlation between two operations on the bonds has to be implemented. Because the lattice is periodic only for every given distance of two bonds a counter is required. Furthermore, the effort is reduced by concentrating on the nearest-neighbor bonds which yield the strongest energy gain. The general estimator proposed by Sandvik in reference [San92] and also described in [Sch04] is given by

$$
\left\langle\prod_{i=1}^{m} H_{b_{i}}\right\rangle=\frac{1}{(-\beta)^{m}}\left\langle\frac{(n-1) !}{(n-m) !} C\left(b_{1}, \ldots, b_{m}\right)\right\rangle,
$$

whereby $C(\ldots)$ is the counter of the above described sub-sequences. For $m=1$ this estimator measures the local potential or kinetic energy depending on the type of $H_{b_{1}}$ (diagonal or off-diagonal). The most relevant case here is $m=2$; three different combinations of diagonal and off-diagonal operators have to be taken into account and result in a net of measurements for dimer correlations on all nearest-neighbor bonds.

\section{error analysis}

The Monte-Carlo results are supposed to be exact with the exception of statistical errors.

To exclude systematic errors due to the random sampling of micro states, the random number generator has to be checked. For this purpose, two completely different types of generators have been used to calculate the model for some parameters and the results were crosschecked, see appendix A and [Kal08]. The presented Monte-Carlo results in this work were produced with an implementation of the Mersenne Twister [MN98] from the Boost library. ${ }^{5}$

For $M$ independent Monte-Carlo runs the estimator for the expectation value and its variance for an observable $A$ are given as [Ber04]:

$$
A=\frac{1}{M} \sum_{i=1}^{M} A_{i} \text { and } \Delta A=\sqrt{\frac{1}{M(M-1)} \sum_{i=1}^{M}\left(A_{i}-A\right)^{2}} .
$$

The $A_{i}$ represent the average values from the each run $i$. For the classical simulations performed in part I at least ten independent runs are computed and the errors for the observables are calculated as described above.

As the quantum Monte-Carlo simulations are much more time consuming, it is worth to put extra effort into the data analysis of each run. The main goal of such an analysis is to detect correlations between consecutive measurements and to avoid an underestimation of the overall error due to these correlations. Two types of error examinations

\footnotetext{
${ }^{5}$ The boost library is freely available at http://www. boost.org.
} 
are distinguished. Firstly, for the binning analysis the time series of length $l$ is divided into an increasing number - powers of two $m=1,2,4, \ldots$ - of bins each containing $k=l / m$ values of the observable yielding an average $A_{i}$ and these are evaluated as described above [equation (3.16)]. The binning analysis is complete if the error $(\Delta A)_{m}$ is converged with respect to the number of bins $m$. Secondly, the jackknife method is usually applied for non-linear functions of measured observables like the fourth-order cumulant given in equation (2.6). A resampling of the different values is applied by omitting single values and estimating the average and the error for the reduced set of correlated measurements. A comparison of the average for different sets yields a reliable error estimate for these observables. Both methods are described in more detail in reference [Ber04] and are implemented within the ALPS libraries [AAC $\left.{ }^{+} 07\right]$.

\section{histogram reweighting}

Sometimes it is useful not only to rely on the average value of an observable but to analyze its distribution at a certain temperature. Therefore, the recording of a histogram or of the complete time series is necessary. However, the knowledge of the distribution of an observable at a certain temperature $T_{0}$ also allows for conclusions about the system at nearby temperatures $T \approx T_{0}$ to be drawn, because the distributions at these temperatures have a large overlap. In particular for large systems, which demand a great amount of computing time, it can be helpful to reweight the measured distribution according to a slightly different temperature. Thereby, informations can be extracted more from the reweighted distribution which were already encoded in the original histogram [Ber04]. The concept relies on an energy-based representation of the partition function

$$
Z_{\text {can. }}=\sum_{E} n(E) \exp \left(-\beta_{0} E\right)
$$

where $n(E)$ signifies the density of states in energy space. This distribution enters the recorded histogram $P_{\beta_{0}}(E)$ at a certain inverse temperature in the Monte-Carlo simulation:

$$
P_{\beta_{0}}(E)=c_{\beta_{0}} n(E) \exp \left(-\beta_{0} E\right) \text { with a normalization constant } c_{\beta_{0}} \text {. }
$$

For a different inverse temperature $\beta_{1}$ the histogram can now be reweighted using this representation as an estimator for $n(E)$ :

$$
P_{\beta_{1}}=P_{\beta_{0}} \frac{c_{\beta_{1}}}{c_{\beta_{0}}} \exp (-\Delta \beta E) .
$$

The interval $\Delta \beta=\beta_{1}-\beta_{0}$ for which a reliable reweighting of the histogram can be achieved, is directly related with to the width of the original histogram, and hence also with fluctuations of the energy, see [Ber04]. It is also possible to involve histograms for different temperatures to provide a broader support of the accessible energy region [GGLM08].

To distinguish different types of phase transitions the shape of the energy histogram at the critical temperature $T_{C}$ can be used. Since the critical temperature is not known in principle for the Ising systems investigated in chapters 5 and 6 , it is complicated to simulate a system exactly at its size-dependent critical temperature. For this reason the reweighting technique will be applied. It is also possible to extract further observables from the new histogram as it provides an estimator for the partition function. 


\section{\begin{tabular}{l|l} 
transfer-matrix calculations & 3.3
\end{tabular}}

A different ansatz is given by the transfer-matrix technique to understand the physics of many particle Hamiltonians with short-range interactions. This technique is based on a factorization of the partition function [Bax82]. The complexity of this factorization depends on the lattice structure and the corresponding interaction terms. For the classical two-spin Ising interactions the exponential function of the Hamiltonian can be written as a product of matrices that only couple smaller subspaces of the complete lattice, e.g., for a one-dimensional periodic chain:

$$
\begin{aligned}
Z_{\text {can. }, N} & =\sum_{\left\{S_{i}= \pm 1\right\}} \exp \left(-\beta J \sum_{i}^{N} S_{i} S_{i+1}\right)=\sum_{\left\{S_{i}\right\}} V\left(S_{1}, S_{2}\right) \ldots V\left(S_{N}, S_{1}\right) \\
& =\operatorname{Tr} V^{N} \text { with } V=\left(\begin{array}{cc}
e^{-\beta J} & e^{\beta J} \\
e^{\beta J} & e^{-\beta J}
\end{array}\right) .
\end{aligned}
$$

The summation over all states thus corresponds to the calculation of the trace of this matrix product. The crucial point of this method is the determination of the subspaces and the corresponding dimension of the matrices that connect the states of two subsequent subspaces. This procedure allows for an exact description of the one-dimensional and twodimensional square lattice Ising models in the thermodynamic limit with only nearestneighbor interactions [Bax82, Isi25, Nig77, Ons44].

For the present case (see section 2.2) with next-nearest neighbor interactions a numerical evaluation is inevitable and limited to finite systems $W \times L$. In principle, the subspace consists of a column of $L$ spins, hence, a matrix $M$ of dimension $2^{L}$ is required to couple the subspaces. The partition function is then given by the trace of the matrix product of $W$ such matrices. The calculation of the product is trivial for a diagonal matrix - and the cyclical behavior of the trace allows for such a transformation - but the diagonalization itself is limited to small $L$. A better approach yields the successive application of the matrix $M$ onto a random initial state, because a matrix vector multiplication is much faster (see also section 3.1). The fact that only a few spins are actually coupled between adjacent columns allows for a decomposition of this matrix vector multiplication into smaller blocks reducing the computational effort. It is not necessary to set up the complete matrix and the memory cost is minimized by saving and altering the weights for all possible states of a single column coupled to its neighboring column only. However, due to the diagonal next-nearest neighbor coupling in the frustrated Ising models this state has to be enlarged at one point by two additional spins. For the details of the implementation please confer [dNNS82] and appendix A.3.

In chapter 5 this technique will be used to calculate the free energy of the frustrated Ising model on a finite cylindric lattice with circumference $L$. The finite-size scaling of the free energy is related to the continuous underlying theory of the lattice model, as will be explained in section 5.2. 


\section{analytical methods

\section{summary}

In this chapter two analytic methods will be outlined which are based on perturbation theory for quantum mechanical systems. Section 4.1 quickly recaps an expansion ansatz for the Hamiltonian of the quantum Heisenberg model around a classical ground state limit. In section 4.2 the concept of perturbative renormalization for a quantum field theory describing the classical two-dimensional Ising model is outlined. In this context some tools from conformal field theory will be introduced which will be required to execute the perturbative computations in chapter 5 .

One basic concept of all three presented computational methods is the calculation of physical observables for finite and rather small systems and a subsequent analysis of the size dependence of the results. This ansatz has become very powerful in the last decades due to increasing computer power and the development of very efficient algorithms. However, an exact closed description of the system in the thermodynamic limit is not achieved. Therefore, the derivation of analytic expressions that are independent on the system size is a desirable task. For complex models, an initial attempt to describe particular observables analytically is a perturbational ansatz. Starting from a known exact description of a part of the model the rest is treated as a small perturbation to that description. The procedure only yields reliable results if the perturbation - the coefficient that scales the strength of the perturbing part of the model - is small compared to all other scales and if the perturbation is well controlled, i.e., if no divergences appear.

\section{Ising series expansion for weak quantum fluctuations [Kog79] 4.1}

For the Heisenberg models discussed in section 2.3 the ground states are known for the limit of zero quantum fluctuations as described by a classical Ising model. These classical ground states yield a good starting point for the derivation of energies for small fluctuations. The Hamiltonian is divided into two parts (see equation (2.19)):

$$
H_{\mathrm{HM}}=\underbrace{\sum_{i, j} J_{i, j}^{z} S_{i}^{z} S_{j}^{z}}_{H_{0}}+\underbrace{\sum_{i, j} \frac{J_{i, j}^{x, y}}{2}\left(S_{i}^{+} S_{j}^{-}+S_{j}^{+} S_{i}^{-}\right)}_{H_{\mathrm{pert}}} .
$$

The unperturbed model $H_{0}$ is the classical Ising model whose ground-state energies and properties are known. The eigen-states of this model - the $S^{z}$ product basis - will be used to calculate corrections to the ground state energy induced by $H_{\text {pert. }}$, i.e., small quantum fluctuations $\left|J^{x, y} / 2\right|<J^{z}$. 
Following the concept of time-independent Rayleigh-Schrödinger perturbation theory in quantum mechanics [Kog79] the perturbative part of the model is rewritten as a series expansion around the unperturbed limit. The individual terms consist of matrix elements between the ground state and exited states that are accessed by applying the perturbation and are divided by the energy cost for the excited state. The computation to first order gives zero because the perturbation $H_{\text {pert. }}$ is purely off-diagonal in the present case. Hence, the first relevant perturbation is second order:

$$
E_{0, \text { pert. }}^{(1)}=0, \quad E_{0, \text { pert. }}^{(2)}=\sum_{k} \frac{\left\langle 0\left|H_{\text {pert. }}\right| k\right\rangle\left\langle k\left|H_{\text {pert. }}\right| 0\right\rangle}{E_{0}-E_{k}} .
$$

The sum is over all accessible states, given by the number of bonds with anti-parallel spins in the ground state. In higher orders the bonds at which the perturbation acts form closed loops and all energies of the intermediate states contribute as a product of $\Delta E$. The derivation of higher order terms also requests some combinatorial considerations on the configuration and multiplicity of these loops. The evaluation of higher-order terms is mostly performed numerically using the concepts of the linked-cluster theorem [OHZ06]. In the present work analytic series up to fourth order and numerical results for the honeycomb lattice up to eighth order [OS11] are compared to Monte-Carlo results.

If the ground state is degenerate - as it is for the Ising model at the maximally frustrated point - an additional step is necessary to avoid a divergence of the series expansion; the basis of the degenerate subspace has to be chosen such that $H_{\text {pert. }}$ is diagonal. However, for the given models these processes are of higher order, i.e., of order $L$ (the linear extent of the lattice) and are therefore negligible in the thermodynamic limit.

The calculation of the perturbed energies is reliable only for small fluctuations, as only then the basic assumption of a perturbed classical state can be valid. The starting point $H_{0}$ for the perturbational approach is wrong for dominating fluctuations. In particular calculations for both classical configurations - Néel state and collinear state - at the highly frustrated point may give different energy corrections and, hence, the stability region may differ from the classical assumption. By comparing both energies at this point the direct transition between these states can be evaluated for non-zero fluctuations up to the given order and a phase boundary is obtained.

\section{\begin{tabular}{l|l} 
conformal field theory & 4.2
\end{tabular}}

«Conformal field theory has found applications in string theory, statistical physics, condensed matter physics, and has been an inspiration for developments in pure mathematics as well.» Statement of the authors in [dFMS97, preface].

The aim of the present section is to motivate the connection of conformal field theory with the classical two-dimensional Ising model and to introduce some concepts and calculational tools for these quantum fields, rather than provide a comprehensive introduction into field theoretical methods which can be found for example in [dFMS97, GNT04, Mus10, Tsv95]. The established concepts and tools will be applied to derive an effective field theory for the frustrated Ising model at criticality for a certain set of parameters in section 5.2 of this thesis. 
side note: conformal transformations

The special class of quantum fields which are interesting in the present context are invariant under conformal transformations. These transformations are characterized by the fact that local angles between different arbitrary curves through the space are conserved. In particular rotations, translations and dilatations (scaling) are included in the group of conformal transformations. The scaling invariance is a crucial point in the calculation of conformal field theories and, hence, every field has an intrinsic scaling dimension $\Delta$. In two dimensions the transformations can be mapped onto analytic functions in the complex plane and are understood very well. In this case the conformal invariance provides useful tools, especially for the derivation of correlation functions that are important in the analysis of phase transitions. For mathematical details on conformal transformations see [dFMS97, chapter 4 and 5].

\section{\begin{tabular}{l|l} 
conformal field theory for a statistical model & 4.2 .1
\end{tabular}}

The evolution of a $d$-1-dimensional quantum model in imaginary time $\tau$ can be rewritten as the statistic evaluation of a $d$-dimensional classical model, see [Mus10, p. 218]:

$$
Z=\sum_{\left\{S_{i}\right\}} \exp \left(-\beta H\left(\left\{S_{i}\right\}\right)=\operatorname{Tr}_{\left\{\phi_{i}\right\}} \prod_{\tau} \exp \left(-\tau H\left(\left\{\phi_{i}\right\}\right)\right) .\right.
$$

The $S_{i}$ represent classical spin variables and the $\phi_{i}$ corresponding quantum fields.

A crucial step from a classical lattice model to a quantum field theory is the continuum limit. Therefore, the dominating length scales in the system have to be analyzed. One length scale is the lattice spacing $a$ and the corresponding range of the interactions, and the other is the correlation length $\xi$. Only in the vicinity of a critical point of a continuous phase transition, where the correlation length is diverging, can the lattice spacing be neglected and scaling invariance is fulfilled. This is why the classical lattice model can be described by a conformal quantum field theory only at criticality [Mus10].

The two-dimensional Ising model can also be described as a quantum field theory of free fermions (see section 4.2.4 below) which is conformally invariant and has a central charge (or conformal anomaly) of $c_{\text {Ising }}=1 / 2$ [dFMS97, chapter 12].

\section{central charge}

his magnitude is characteristic for a conformal quantum field theory in two dimensions and can be derived from the correlation function of the stress-energy tensor (of the quantum field theory) with itself, see [Mus10, p. 259 and 329]. The central charges of different conformal theories add up (based on the extensivity of the energy [dFMS97, section 5.4]) and for two independent Ising models it holds that $c_{2 \times \text { Ising }}=1 / 2+1 / 2=1$. Furthermore, there exists a theorem on the behavior of the central charge for two-dimensional field theories stating that under certain conditions $c$ can only decrease along the flow of a renormalization group transformation, see [Mus10, p. 504] and [Zam86]. This will be important in the context of criticality and corresponding universality classes because every unitary theory with $c<1$ yields a universality class with constant universal exponents. It is only possible to obtain a phase transition with varying critical exponents for a conformal field theory with $c \geq 1$ [Gin88]. 


\section{\begin{tabular}{l|l} 
perturbative renormalization & 4.2 .2
\end{tabular}}

The concept of renormalization group transformations in the context of continuous phase transitions relies on the fact that the correlation length $\xi$ is diverging at the critical point. Thus, the idea of renormalization is based on the scaling invariance of the system at its critical point and yields a flow diagram with different parameters in the model, such as temperature and coupling constants as coordinates [Car96, Gol93]. Flows start and end at fixed points and these represent field theories with a definite phase transition behavior, i.e., representing a universality class. The exact positions of these points and the flow diagram itself result from the solution of the renormalization group equations. However, a rough analysis of the scaling parameters and their corresponding fields is sometimes sufficient to identify the fixed-point action of the underlying field theory [Car96].

Each scaling parameter scales with a set of fields from the conformal field theory and their importance for the phase transition behavior depends on the intrinsic scaling dimension $\Delta$ of these fields. Three cases are distinguished and in two dimensions for these cases holds: $\Delta<2$ is relevant and drives the system away from the original fixed point to a new fixed point; for $\Delta=2$ the coupling is marginal and a careful analysis of the renormalization group flow may be necessary; fields with $\Delta>2$ are irrelevant and the flow along the corresponding parameter ends in the original fixed point.

The reference to an original fixed point emphasizes that the starting point for the perturbative renormalization analysis is a well known model which in the present case is a theory of two independent Ising models. The coupling of these two models is then treated perturbatively. While the first order perturbation is given directly by the derivation of the continuum limit of the coupled lattice models, higher order perturbations are calculated using the tools from conformal field theory, as outlined below.

The set of fields derived from the perturbation can be compared to known conformal field theories to identify the most relevant fixed point. Alternatively the renormalization group equations have to be solved to identify the fixed point action.

For more details on the renormalization group concepts please confer [Car96, Gol93].

$$
\begin{array}{l|l}
\text { operator product expansion } & 4.2 .3
\end{array}
$$

One powerful tool for explicit calculations in conformal field theories is the operator product expansion. Basic concepts of quantum field theory and the consequences of the underlying conformal symmetries are unified in a set of calculational rules for the evaluation of correlation functions of multiple quantum fields. ${ }^{1}$

A detailed derivation of these tools in two dimensions is given, for example in [dFMS97, chapter 5 and 6]. Here only a short road-map is presented that outlines the crucial steps for the computation of the correlation functions. In a first step the two-point correlation functions are constructed on the basis of the underlying conformal symmetries and the scaling dimensions of the correlated fields. They have to fulfill the rotational and translational invariance and are connected to the scaling behavior of the original

\footnotetext{
${ }^{1}$ The explicit computation of the correlators which is not shown here can be performed either in pathintegral formalism or in operator formalism. However, in operator formalism additional mathematical methods can be employed [dFMS97, chapter 6]. The tools presented below are based on the operator formalism, and hence the fields are referred to as operators from now on.
} 
fields. ${ }^{2}$ The scaling dimension of the fields can be extracted from the critical exponent of the correlation functions, e.g., for a correlation function for identical spin operators $\sigma$ at $(z, \bar{z})$ and $(w, \bar{w})$

$$
\langle\sigma(z, \bar{z}) \sigma(w, \bar{w})\rangle=\frac{C}{|z-w|^{\eta}}, \quad C=\mathrm{constant}
$$

holds $\eta=1 / 4$ (critical exponent for the two-dimensional Ising model), and hence $\Delta_{\sigma}=$ $\eta / 2=1 / 8$ [dFMS97, p. 221]. Furthermore, the conformal dimensions $h$ and $\bar{h}$ are defined for each operator with

$$
\Delta=h+\bar{h} \quad \text { and } \quad s=h-\bar{h}
$$

where $s$ is the conformal spin.

Throughout this work the focus lies on spin operators $\sigma$, energy operators $\varepsilon$ (which are present in all theories and couple to the temperature), and their derivatives. The conformal dimensions for these operators are summarized in table 4.1, confer also [BPZ84a, Gin88].

\begin{tabular}{c|cccc}
\hline operator & $h$ & $\bar{h}$ & $\Delta$ & $s$ \\
\hline$\sigma(z, \bar{z})$ & $1 / 16$ & $1 / 16$ & $1 / 8$ & 0 \\
$\varepsilon(z, \bar{z})$ & $1 / 2$ & $1 / 2$ & 1 & 0 \\
$\partial_{z}$ & 1 & 0 & 1 & 1 \\
$\partial_{\bar{z}}$ & 0 & 1 & 1 & -1 \\
\hline
\end{tabular}

table 4.1: Conformal dimensions $h$ and $\bar{h}$ for operators needed in the context of the twodimensional Ising model. In addition the resultant magnitudes $\Delta$ (scaling dimension) and $s$ (conformal spin) are listed, see equation (4.5).

For the calculation of higher-order correlators fusion rules are applied [dFMS97, chapter 8.4 and 12$]$

$$
\begin{aligned}
{[\sigma][\sigma] } & \rightarrow[\mathbb{1}]+[\varepsilon] \\
{[\sigma][\varepsilon] } & \rightarrow[\sigma] \\
{[\varepsilon][\varepsilon] } & \rightarrow[\mathbb{1}]
\end{aligned}
$$

and expressed as an expansion around the two-point correlator (here up to first order in the partial derivative $\left.\partial_{\{w, \bar{w}\}}\right)[\mathrm{dFMS97}$, chapter 12]:

$$
\begin{aligned}
\sigma(z, \bar{z}) \sigma(w, \bar{w})= & C_{\sigma \sigma \mathbb{1}}|z-w|^{-\frac{1}{4}}+C_{\sigma \sigma \varepsilon}|z-w|^{\frac{3}{4}} \varepsilon(w, \bar{w}) \\
& +\frac{C_{\sigma \sigma \varepsilon}}{2}(z-w)^{\frac{11}{8}}(\bar{z}-\bar{w})^{\frac{3}{8}} \partial_{w} \varepsilon(w, \bar{w})+\text { Hermitian conjugate } \\
\sigma(z, \bar{z}) \varepsilon(w, \bar{w})= & C_{\sigma \varepsilon \sigma}|z-w|^{-1} \sigma(w, \bar{w}) \\
& +\frac{C_{\sigma \varepsilon \sigma}}{2}(z-w)^{\frac{1}{2}}(\bar{z}-\bar{w})^{-\frac{1}{2}} \partial_{w} \sigma(w, \bar{w})+\text { H.c. } \\
\varepsilon(z, \bar{z}) \varepsilon(w, \bar{w})= & C_{\varepsilon \varepsilon \mathbb{1}}|z-w|^{-2}
\end{aligned}
$$

\footnotetext{
${ }^{2}$ For two-dimensional field theories the conformal invariance imposes constraints on the correlation functions that are also called Ward identities [dFMS97, chapter 5].
} 
where the $C$ s are structure constants which can be derived from certain limits of higherorder correlators [dFMS97, chapter 12]. In particular $C_{\varepsilon \varepsilon \mathbb{1}}=c_{\text {Ising }}=1 / 2$. The other constants are chosen to be

$$
C_{\sigma \sigma \mathbb{1}}=1 \text { and } C_{\sigma \sigma \varepsilon}=\frac{1}{2}=C_{\sigma \varepsilon \sigma} .
$$

Furthermore, it is notable that the scaling behavior in the operator product expansion is conserved, i.e., if the overall conformal dimensions $h$ and $\bar{h}$ of the operators on the left and right hand sides of the equations (4.9)-(4.11) differ, the scaling exponent of the corresponding variables $(z, w)$ and $(\bar{z}, \bar{w})$, are changed accordingly.

$$
\begin{array}{l|l}
\text { statistical models } & 4.2 .4
\end{array}
$$

In the context of this work the mapping of two-dimensional statistical models at criticality onto conformal field theories is employed. Two models in particular and their corresponding field theories will be important.

\section{Ising model}

The two-dimensional Ising model can be mapped onto a one-dimensional quantum spin chain which again can be transformed into a model of free fermions using the JordanWigner transformation [dFMS97, GNT04, Mus10, Tsv95].

Thus, the Ising model can also be described by an action $\mathcal{A}$ of a field theory of free fermions $\psi$ and an additional mass term:

$$
\mathcal{A}_{\text {Ising }}=\int d z d \bar{z}\left(\psi \partial_{\bar{z}} \psi+\bar{\psi} \partial z \bar{\psi}\right)+m \int d z d \bar{z} \psi \bar{\psi}
$$

The mass term represents the energy $(\varepsilon=\psi \bar{\psi})$ and is tuned by its scaling factor $m$ exactly to zero at the critical temperature, i.e., $m \propto\left(T-T_{C}\right)$ [dFMS97].

The fermionic theory will not be further discussed in this work. However, the introduced field theoretical methods will be used in chapter 5 to calculate the underlying field theory of an isotropic frustrated model at criticality for large $J_{2}>J_{1}$. The limit of this frustrated model is given by two independent Ising models.

\section{Ashkin-Teller model}

The field theory of this lattice model (introduced in section 2.2.2) is given by two copies of the Ising field theory from equation (4.13) plus an additional marginal energy-energy coupling

$$
\mathcal{A}_{\mathrm{AT}}=\mathcal{A}_{\mathrm{Ising}, \mathrm{A}}+\mathcal{A}_{\mathrm{Ising}, \mathrm{B}}+k \int d z d \bar{z} \varepsilon_{A} \varepsilon_{B}
$$

The central charge is given by $c_{\mathrm{AT}}=1$ [Gin88] which together with marginal coupling allows for phase transitions with continuously varying critical exponents [FQS84, KW71]. 


\section{ordering processes in the 2D Ising model}





\section{analysis of the phase transition for the Ising model on the frustrated square lattice $\mid 5$}

The main results of this chapter were published under the same title in Physical Review B [KHM11]. Coauthors of this work were Andreas Honecker from the University of Göttingen as scientific advisor and Marion Moliner from the Karlsruher Institut für Technologie. The numerical work (Monte-Carlo and transfer-matrix calculations) was performed by me. Marion Moliner presented a first ansatz for the continuous theory; all subsequent calculations for the continuum limit and the operator product expansion for higher-order perturbative terms were performed by me and crosschecked with Marion Moliner's results.

It may be that some parts of the following chapter resemble the manuscript of reference [KHM11] which has been written together with the above mentioned coauthors.

\section{abstract}

The frustrated $J_{1}-J_{2}$ Ising model on a square lattice has two different ground states as described in section 2.2. In this chapter the finite-temperature phase transition from the high-temperature paramagnetic phase to the collinear ordered ground state will be analyzed by means of Monte-Carlo simulations - in particular energy histograms and spinspin correlation functions will be presented in section 5.1. Furthermore, the underlying conformal field theory of the model at criticality, i.e. $T=T_{C}\left(J_{2} / J_{1}\right)$ will be characterized using transfer-matrix calculations and a perturbative renormalization calculation in the limit of small $J_{1}<J_{2}$ by means of conformal field theory.

As a result a phase transition scenario is obtained which includes a weak first order transition for intermediate values of $J_{1} / 2<J_{2}<J_{1}$ and a continuous phase transition with varying critical exponents for larger values of $J_{2} / J_{1} \rightarrow \infty$. This latter phase transition is described by an underlying Ashkin-Teller field theory.

For convenience the Hamiltonian of the frustrated isotropic Ising model on the square lattice is given here again:

$$
H_{\text {Ising }}=J_{1} \sum_{\mathrm{NN}} S_{i} S_{j}+J_{2} \sum_{\mathrm{NNN}} S_{i} S_{j}
$$

Both coupling constants $J_{k}$ are positive and the antiferromagnetic exchange on nearest and next-nearest neighbor bonds introduces a competition of the Néel ordered and collinear ordered configuration at a critical point $J_{2}=J_{1} / 2$. The focus will be on the thermal phase transition for $J_{2}>J_{1} / 2$ whose character has been under debate since the 1980s.

An initial analysis of the model with Monte-Carlo simulations exhibits the emergence of a first-order phase transition for an intermediate range of frustration $J_{1} / 2<J_{2} \lesssim 0.9 J_{1}$. However, it is also observed that the length scales for increasing $J_{2}$ are diverging and, hence, further analysis of finite lattices with Monte-Carlo simulations would not be promising. That is why, in section 5.2, the field theoretic point of view is introduced. To characterize the underlying field theory of the frustrated model at criticality, firstly a calculation 


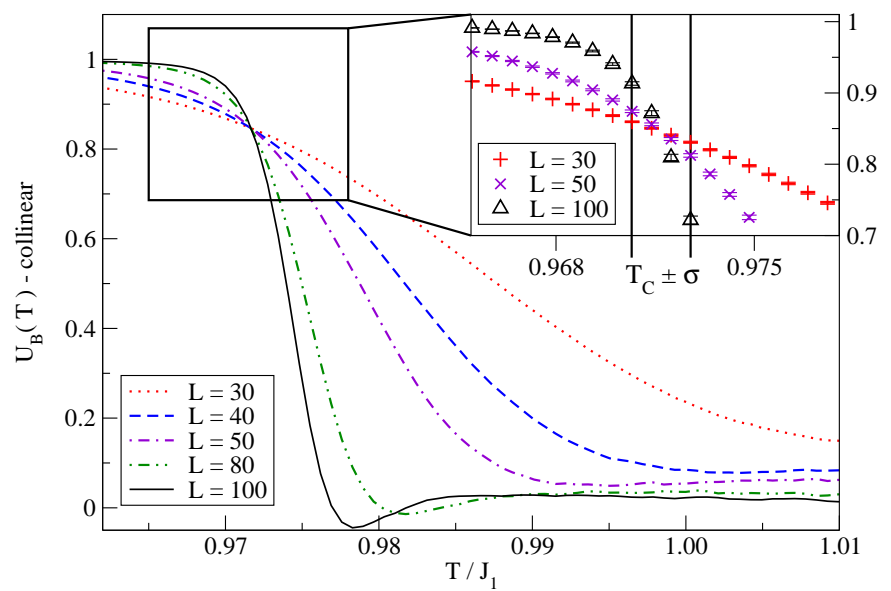

figure 5.1: (Slightly different version published in [KHFP08]). Binder cumulants for the collinear order parameter versus temperature at $J_{2}=0.6 J_{1}$. The intersection point of the cumulants for different linear lattice sizes $L$ yields an estimation of $T_{C}=0.9715(10) J_{1}$, as shown clearly in the inset.

of the central charge of the finite lattice system is performed before the continuum limit is computed at $J_{1}=0$, which is then subject to a perturbative renormalization procedure for increasing $J_{1} \nearrow J_{2}$.

\section{\begin{tabular}{l|l} 
Monte-Carlo simulation & 5.1
\end{tabular}}

The simulation of the frustrated model was performed using a single-spin flip Metropolis update with an additional line-flip update as was described in section 3.2.1. For an optimized thermalization process and high-quality data an exchange Monte-Carlo update [Han97, HN96, KTHT06, also see section 3.2.1] was also implemented. The simulations were run on large-scale clusters using OpenMP and MPI. The statistical errors of the data were obtained from multiple independent Monte-Carlo runs.

\section{phase diagram}

A first step for the analysis of a phase transition is the exact determination of the critical point for different values $J_{2} / J_{1}$. For the frustrated Ising model this critical temperature is only known analytically in the limits $J_{2}=0$ and $J_{1}=0$ where the value of $T_{C}$ is given
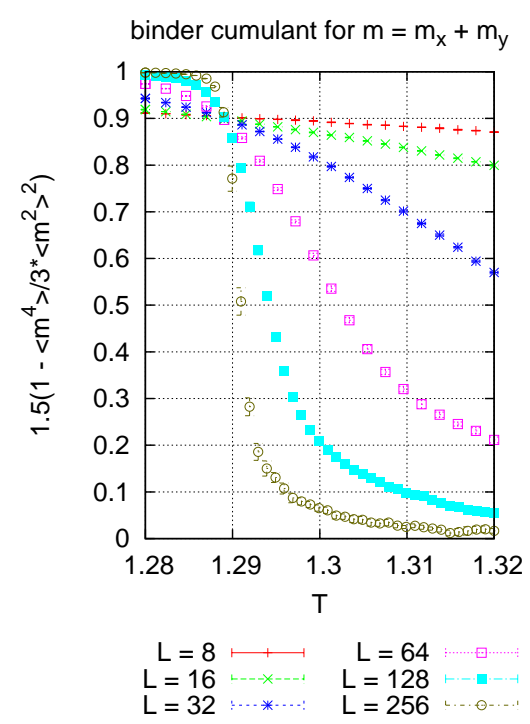
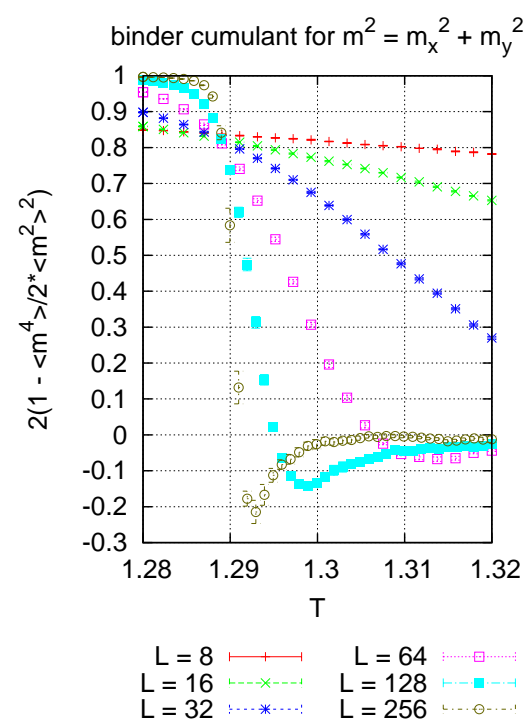

figure 5.2: Two different types of Binder cumulants for the different collinear order parameters versus temperature at $J_{2}=0.7 \quad J_{1}$. The intersection points yield the same critical temperature $T_{C}=1.289(1) J_{1}$ but different values for $U_{4}\left(T_{C}\right)$. 


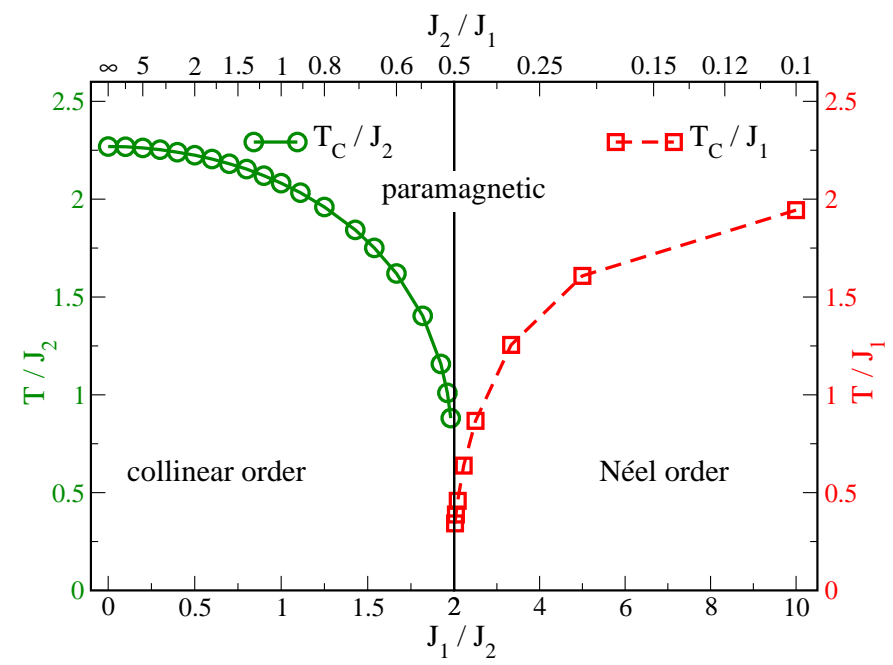

figure 5.3: (Published in [KHM11].) Critical temperatures for the phase transition from the paramagnetic to the magnetically ordered phases divided by the strength of frustration $J_{1} / J_{2}$. The energy scale of the temperature is adapted for $J_{1} / J_{2}<2$ to $J_{2}$ and for $J_{1} / J_{2}>2$ to $J_{1}$. Note that the frustration is also given in units of $J_{2} / J_{1}$ on the upper $x$ axis.

in the relevant energy scale $J_{1}$ and $J_{2}$, respectively, i.e., $T_{C}=2.269 J_{1,2}$ [Ons44]. For all other values of $J_{2} / J_{1}$ the determination can be achieved numerically by calculating the intersection point of the Binder cumulant (given in equation (2.6)) of the appropriate order parameter for different lattice sizes $L \times L$. In the present case the order parameters for the Néel and collinear phase are given by the structure factors which were introduced in chapter 2. As an example the Binder cumulants and the estimation of the critical temperature is shown in figure 5.1 for $J_{2}=0.6 J_{1}$ and yields $T_{C}=0.9715(10) J_{1}$. The sum of the two equivalent structure factors $m_{\text {coll., } x}$ and $m_{\text {coll.,y }}$ from equation (2.12) is used as order parameter.

By using a different order parameter, namely the sum of squares $m^{2}=m_{\text {coll. }, x}^{2}+m_{\text {coll., } y}^{2}$ the definition of the Binder cumulant has to be adapted to the distribution of the moments, see also [JSS12]. Thus, the shape of the cumulants is changed in the vicinity of the critical temperature. The intersection point, however, yields the same critical temperature but a different value for the cumulant, as is shown in figure 5.2. This demonstrates that both order parameters serve for an accurate detection of the transition temperature. However, an analysis of the phase transition by referring to universal amplitudes of the Binder cumulant at the critical point is not straightforward [Sel06, JSS12].

The critical temperatures for $0 \leq J_{1} \leq 10 J_{2}$ (or $0.1 J_{1} \leq J_{2}<\infty$ ) are summarized in the phase diagram in figure 5.3. The energy scale of the transition lines are adapted to the relevant scale, i.e. for the transition to the Néel phase to the nearest-neighbor interaction $J_{1}$ and to $J_{2}$ for the collinear phase transition.

$$
\text { phase transition analysis } \mid 5.1 .1
$$

The phase transition from the paramagnetic high-temperature phase to the Néel ordered region is continuous for all ratios of $J_{2} / J_{1}<0.5$, as was already shown, for example, by Landau and Binder [LB05]. The computation of critical exponents and histograms for these parameters was presented also in [Kal08, KHFP08] and confirmed the earlier statements that the critical model and, hence, the phase transition belongs to the twodimensional Ising universality class, which is based on the limiting case $J_{2}=0$ [Gol93].

The calculation of critical exponents for larger values $J_{2} / J_{1}>0.5$ yields controversial results [KHFP08] and, as introduced in sections 1.1 and 2.2 , the phase-transition sce- 


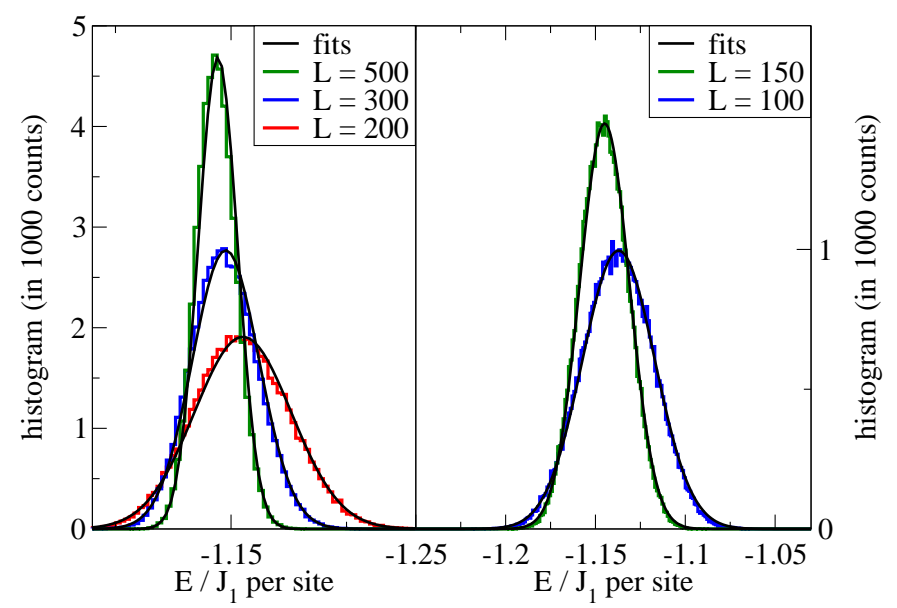

figure 5.4: Energy histograms for a continuous phase transition shown for $J_{2}=0.3 J_{1}$ and increasing linear lattice sizes L. All histograms can be fitted rather accurately by Gaussian distributions (black lines).

nario is under debate. Energy histograms and correlation functions will be presented for different values of $J_{2} / J_{1}>0.5$ to analyze the nature of this phase transition.

\section{histograms}

The shape of histograms for first order and continuous phase transitions was discussed in section 2.1: For a continuous phase transition a nearly Gaussian distributed energy is expected at the critical temperature $T_{C}$. The energy histograms for a value of $J_{2}=0.3 J_{1}$ and different lattice sizes $L$ are shown as examples in figure 5.4. For all $L$ a Gaussian fit resembles the shape of the histogram quite accurately. However, in the case of a first order transition the shape of the energy histogram also contains the information about the energy discontinuity at $T_{C}$ which is manifested as a latent heat and is represented by a two-peak structure. Such a two-peak structure was found in the frustrated Ising model for the phase transition from the paramagnetic phase to the collinear ordered phase, i.e., for $J_{2}>J_{1} / 2$. The histograms for small values $J_{2}=0.6 J_{1}$ and $J_{2}=0.65 J_{1}$ were presented in references [KHFP08, KHFP09] and clear evidence for a two-peak structure and first order transition was given. However, already for $J_{2}=0.7 J_{1}$ simulations with $L \leq 500$ were not sufficiently large to resolve two-peaks structures in the energy distribution.

Since López et al. [MLAGS93, MLAGS94] claim the first-order transition scenario to be valid up to $J_{2} \simeq 1.1 J_{1}$ the recording of histograms for larger values of $J_{2}$ was necessary. Therefore, larger lattices needed to be simulated and the results for $J_{2}=0.8 J_{1}$ with $L=1000$ and $J_{2}=0.9 J_{1}$ with $L=2000$ are shown in figure 5.5. It is worth

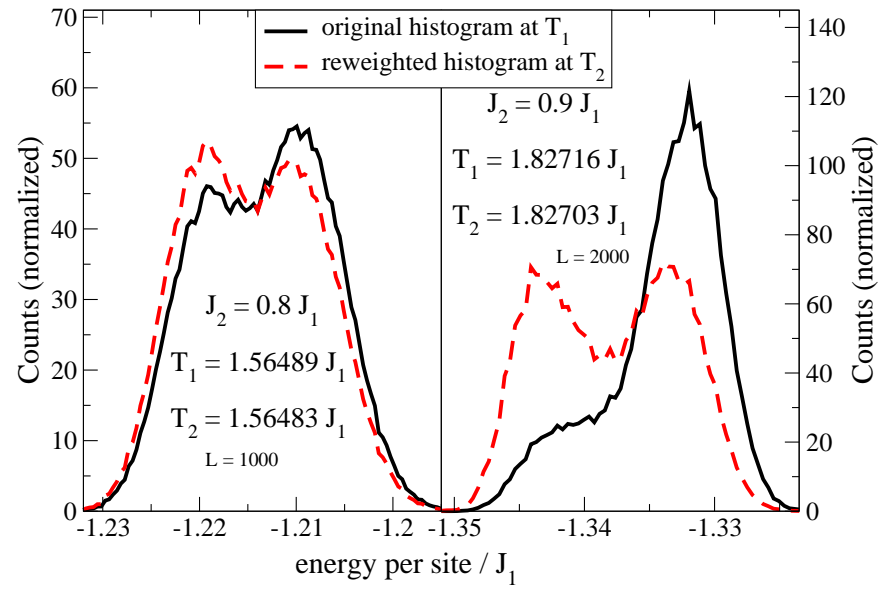

figure 5.5: (Published in [KHM11].) Energy histograms (solid black lines) for two values of $J_{2}=0.8 J_{1}$ (left) and $J_{2}=0.9 J_{1}$ (right) for a $L=1000$ (left) and $L=2000$ (right) lattice at temperature $T_{1}$. The reweighted histograms (dashed red lines) are more symmetric because the temperatures $T_{2}<T_{1}$ are closer to the size-dependent critical temperature. The two-peaked structure emphasizes the first-order character of the phase transition. 


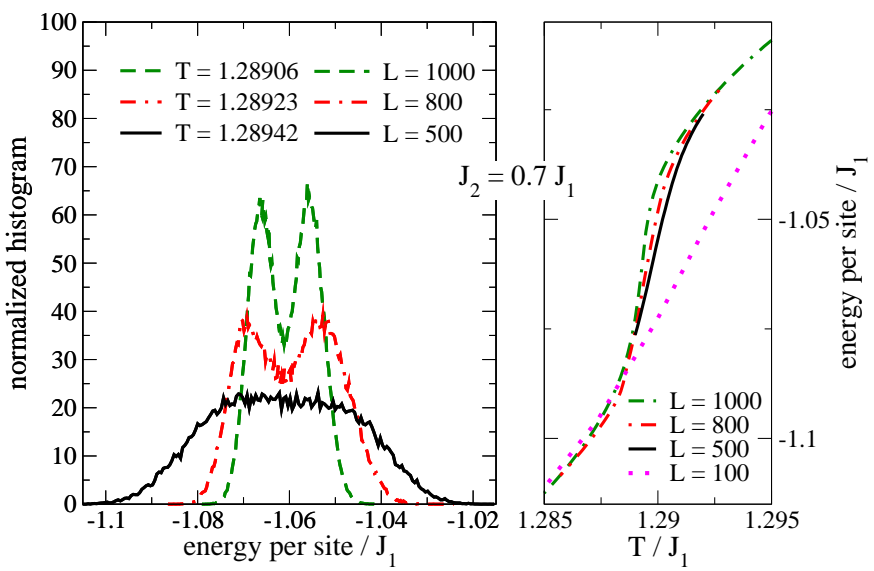

figure 5.6: Evolution of the twopeak structure in the histogram for $J_{2}=$ $0.7 J_{1}$ (left) and the energy (right). The depth of the minimum between the two peaks increases with system size and the overall shape is stable with increasing $L$. Furthermore the energies show increasingly steeper slopes for increasing $L$ which also indicates a first-order transition.

mentioning that for a small step in the parameter space $\left(0.1 J_{1}\right)$ it is necessary to double the linear size of the simulated lattice to achieve a similar resolution for the recorded histogram. The shapes of these histograms show a strong deviation from the almost Gaussian shape that is expected for a continuous phase transition, yet for the simulated temperatures the structure is not symmetric. For this reason, reweighted histograms are also presented (as dashed red lines) for slightly lower temperatures, i.e., the size-dependent transition temperatures. The histograms are thus shifted to lower energies and exhibit a more distinct and symmetric two-peak structure. The simulation and recording of a new histogram for this temperature would have been too time consuming and, hence, the standard reweighting technique [Ber04, GGLM08, see also section 3.2.1] was applied to confirm the first-order character of the phase transition.

In a recent publication by Jin et al. [JSS12] it is claimed that the first-order transition is only stable for $0.5 J_{1}<J_{2} \lesssim 0.67 J_{1}$ and indications for discontinuous phase transitions for larger values of $J_{2}$ are not trustworthy. They used mainly arguments stemming from the universality of Binder cumulants and doubt the stability of the two-peak structure in the histograms. Therefore, the finite-size scaling of the two-peak pattern in the energy histograms is analyzed in the left-hand side of figure 5.6. In detail the evolution of two distinct peaks in the histograms is shown at $J_{2}=0.7 J_{1}$ and the minimum in between increases for larger systems $(500 \leq L \leq 1000)$. In addition, the development of the energies in the direct vicinity of the critical temperature is shown for different lattice sizes. The increasing steepness of the slope also indicates a first-order transition.

The above presented data for $0.5 J_{1}<J_{2} \leq 0.9 J_{1}$ gives evidence that the phase transition is discontinuous in this region of the phase diagram. However, for larger values of $J_{2}$ the increasing length scales do not allow for such an analysis of the histograms and, hence, no concrete statement about the nature of the phase transition is possible from such an analysis.

\section{correlation functions}

Up to now the phase transition has been investigated for increasing ratios $J_{2} / J_{1}>0.5$. However, a different perspective can be adopted by analyzing the transition for increasing $0 \leq J_{1} / J_{2}<2$. In the limit of $J_{1}=0$ and $J_{2}>0$ the original square lattice can be interpreted as two separate square lattices - the sublattices $A$ and $B$ - rotated by an angle $\pi / 4$ with a rescaled lattice spacing $a=\sqrt{2} a^{\prime}$ whose nearest-neighbor bonds are the original next-nearest neighbor bonds (sketched in figure 5.10 below). The physics of the model is then described by two identical copies of the simple square lattice Ising model 


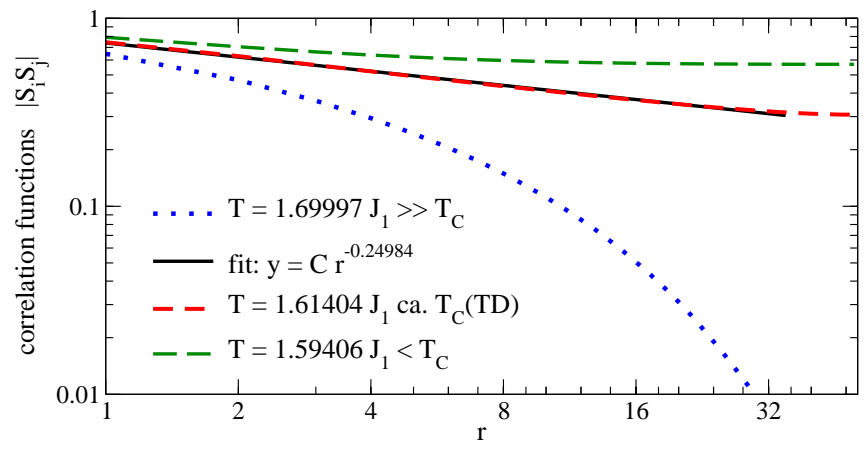

figure 5.7: Absolute values of the correlation functions in a doubly logarithmic scale for $J_{2}=0.2 J_{1}$ on a $100 \times$ 100 square lattice. An algebraic fit at the size-dependent transition temperature yields $\eta=0.2498(7)$.

with a coupling $J_{2}$. Hence, as mentioned above and shown in the phase diagram (figure 5.3) the transition temperature is known exactly; the phase transition is continuous and exhibits the 2D-Ising critical exponents. One of these exponents $(\eta)$ describes the scaling of the correlation functions at the transition temperature [Gol93]:

$$
G(r)_{T_{C}}=S_{i} S_{j} \propto r^{-\eta} \text { with } r=\left|\mathbf{r}_{i}-\mathbf{r}_{j}\right|
$$

For Monte-Carlo simulations on finite periodic lattices $(L \times L)$ the scaling can only be evaluated for an intermediate region of $r$ since for $r \nearrow L / 2$ the correlations are enhanced due to the periodic boundary conditions. For temperatures $T>T_{C}$ an exponential decay is expected and in the ordered phase, i.e., $T<T_{C}$, a fast saturation towards a finite value that reflects the long-range order should be found.

In figure 5.7 the absolute correlation functions $\left|S_{i} S_{j}\right|$ are plotted on a doubly logarithmic scale versus the distance $r$ for the case of two coupled Ising models that undergo an Ising-phase transition from the paramagnetic to the Néel phase $\left(J_{2}=0.2 J_{1}\right)$. The critical exponent $\eta=0.2498(7)$ is extracted via an algebraic fit and the result is in very good agreement with the Ising value $\eta_{\text {Ising }}=0.25$ [Gol93].

For the decoupled case the correlations are shown in figure 5.8a separately for $i$ and $j$ being part of the same sublattice $\left(r_{\text {even }}\right.$, top) and different sublattices ( $r_{\text {odd }}$, bottom) in a

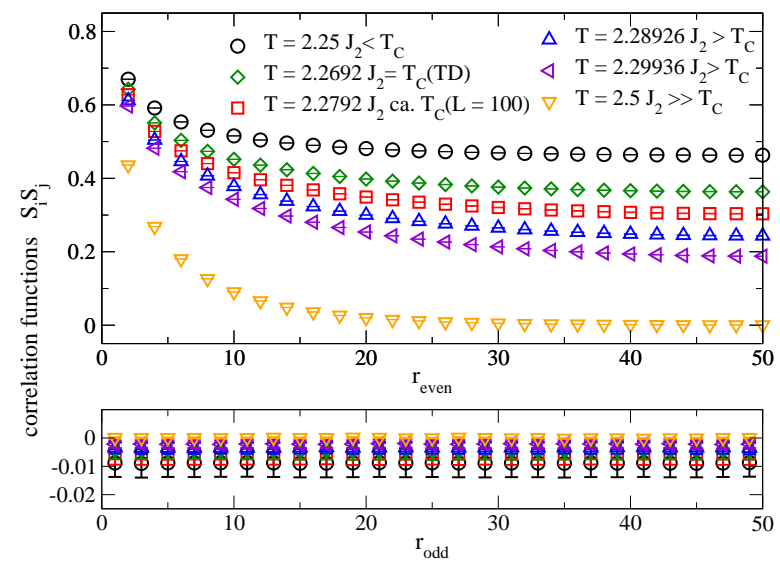

(a) linear scale for all distances

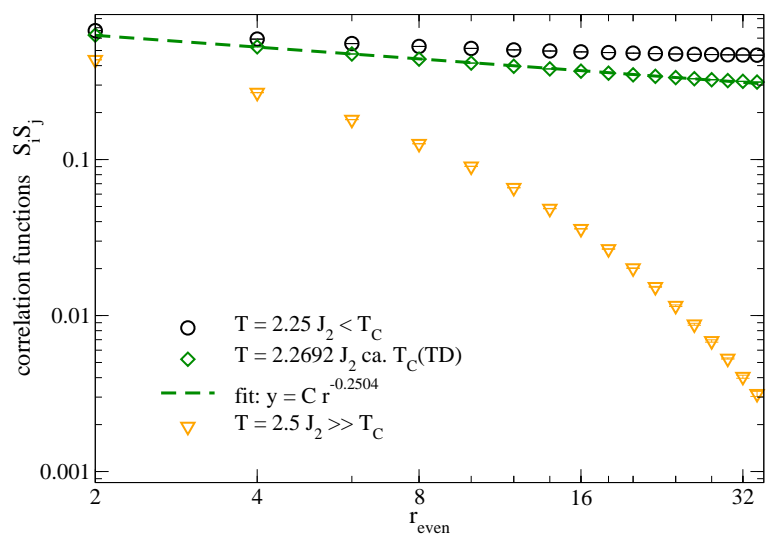

(b) doubly logarithmic scale for $r_{\text {even }}$

figure 5.8: A joint plot for both sublattices for the decoupled models, i.e., $r_{\text {even }}$ and $r_{\text {odd }}$ are not meaningful. Thus, the correlations are plotted separately in a linear scale (a) which clearly shows that the models are independent. Plots for several temperatures are shown and in the upper panel a clear difference can be seen between the ordered and disordered state. (b) The scaling analysis of the correlations versus $r_{\text {even }}$ in a doubly-logarithmic plot reveals the Ising character of the phase transition; the critical exponent $\eta=0.2504(9)$ is extracted $(L=100)$. 


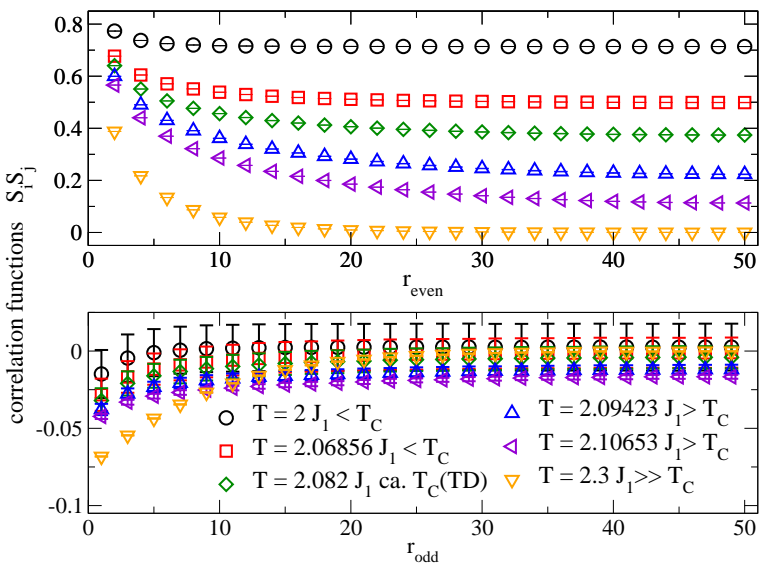

(a) linear scale for all distances

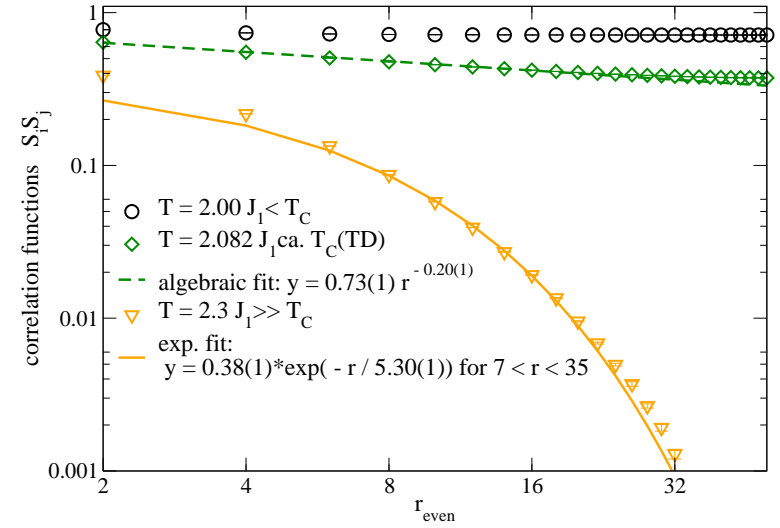

(b) doubly logarithmic scale for $r_{\text {even }}$

figure 5.9: (Published in [KHM11].) Spin-spin correlation functions in one direction at $J_{1}=J_{2}$ for a $100 \times 100$ periodic lattice. Maximal distance is thus given by $r=50$. Top left: Correlations inside the same sublattice for different temperatures around $T_{C}$. Bottom left: Correlations between spins on different sublattice sites on a magnified scale. The correlations decay rapidly and go to zero for all temperatures. Hence, no long-range correlation is observed between the two sublattices. Right: Doubly logarithmic plot of the correlation functions inside the same sublattice for three exemplary temperatures and related fits [for the highest temperature (yellow triangles) values for $r>32$ are not shown]. The critical exponent $\eta=0.20(1)$ is significantly below the Ising value.

linear scale; the decoupling is clear. Again the critical exponent can be derived from the behavior of the correlation functions at distances $r_{\text {even }}$ at the transition temperature and yields $\eta=0.2504(9)$, where the error stems from the algebraic fit.

Following now the limit $J_{1} \nearrow J_{2}$ (see figure 5.3, bottom panel) the critical exponent is first stable under the introduction of competing interactions on nearest-neighbor bonds, i.e., $\eta\left(J_{1} / J_{2}=0.2\right)=0.256(7)$. For the point $J_{1}=J_{2}$ the correlation functions are shown in figure 5.9. Comparing the separately plotted correlations in figure 5.9a with the results given in figure $5.8 \mathrm{a}$ an enhancement in the inter-lattice coupling is clearly visible. Nevertheless, for increasing distances the coupling tends towards zero for all temperatures, i.e., the two sublattices are also nearly decoupled in the ordered phase. This behavior is observed for all values $J_{1}<2 J_{2}$, i.e., for all transitions to the collinear phase. Hence, the doubly logarithmic plot in figure 5.9b contains only correlations for $r_{\text {even }}$ and the evaluation of the phase transition is also performed for these correlations. As shown in figure $5.9 \mathrm{~b}$ the scenario of an Ising-type phase transition is no longer valid because $\eta=0.20(1)$ differs significantly from the Ising value.

In conclusion, the Monte-Carlo data yield a clear picture only for $0.5 J_{1}<J_{2} \lesssim 0.9 J_{1}$ where a first-order phase transition scenario is established by the doubly peaked structure of the energy histograms. For larger values of $J_{2}$ the analysis of the correlation functions indicates a decoupling of the two sublattices and a continuous phase transition. However, a detailed examination of the scaling behavior at the critical temperature and a reliable calculation of critical exponents are hampered by large crossover scales. 


\section{conformal field theory}

Because the system is exactly described by two decoupled two-dimensional Ising models on sublattices $A$ and $B$ in the limit $J_{1}=0$, the critical behavior can be tackled by conformal field theory, see section 4.2. A conformal field theory is characterized by its central charge $c$ which in this particular case of two Ising models equals $c=1 / 2+1 / 2=1$, see section 4.2.1.

The identification of the underlying field theory for parameters $0<J_{1} \lesssim J_{2}$ by computing the central charge can help to understand the phase transition scenario of the frustrated Ising model.

\section{\begin{tabular}{l|l} 
transfer matrix & 5.2 .1
\end{tabular}}

In the 1980s it was shown that, for a lattice model, the free energy $f$ per site of a cylinder of infinite length and finite circumference $L$ shows a finite-size scaling in $1 / L$ with a proportionality factor depending on the central charge of the corresponding field theory [Aff86, BCN86]:

$$
f=b-c \frac{\pi T_{C}}{6 L^{2}}+\mathcal{O}\left(L^{-4}\right), \quad b=\text { const. . }
$$

To calculate the free energy, Monte-Carlo simulations are not suitable because they cannot be used to directly sample the entropy. Therefore, a transfer-matrix algorithm has been implemented [Bax82, dNNS82, see also section 3.3 and appendix A.3].

\begin{tabular}{cc|cc}
\hline$J_{2} / J_{1}$ & $c$ & $J_{1} / J_{2}$ & $c$ \\
\hline 0.0 & $0.4999(1)$ & 0.9 & $1.0450(6)$ \\
0.2 & $0.4994(3)$ & 0.8 & $1.0327(5)$ \\
0.4 & $0.4923(7)$ & 0.7 & $1.0232(4)$ \\
0.6 & {$[1.5811(18)]$} & 0.6 & $1.0159(4)$ \\
0.8 & {$[1.1273(10)]$} & 0.4 & $1.0062(5)$ \\
0.9 & {$[1.0863(7)]$} & 0.2 & $1.0012(4)$ \\
1.0 & $1.0613(6)$ & 0.0 & $0.9996(1)$ \\
\hline
\end{tabular}

table 5.1: Central charge of the underlying field theory for different $J_{2} / J_{1}$ (left) and $J_{1} / J_{2}$ (right) calculated with a transfer-matrix computation of the free energy. The width of the computed systems satisfies $L \leq 22$. In parentheses $c$-values for $J_{1} / 2<J_{2}<J_{1}$ are included where according to the Monte-Carlo analysis the phase transition is of weakly first order.

The free energy for systems of size $L \times B$ were calculated. Although the circumference of the cylinder was limited to $L \leq 22$, because of the exponential growth of the computational effort, the length could be chosen easily up to $B=10000$ (linear scale). A finite-size scaling of the free energy at the critical temperature $T_{C}$ (obtained by MonteCarlo simulations, see figure 5.3) and the fit with the equation (5.3) yield an estimate of the central charge plus error bar for different sets of parameters. The results for different $J_{2} / J_{1}$ are summarized in table 5.1. For large values of $J_{2}$ (or small values of $J_{1} / J_{2}$, bottom of right column) the central charges converge to the value of two independent Ising models, $c=1$. Although at intermediate parameters $0.6 J_{2} \leq J_{1} \leq J_{2}$ the values for $c>1$ already suffer finite-size problems, the results from the fits tend towards $c=1$. However, 
for decreasing $J_{2}<J_{1}$ (bottom of left column) they do not converge, and the central charge $c$ is increasing which is not allowed by the $c$ theorem which states that the central charge cannot increase under the influence of a renormalization-group transformation if the corresponding field theory is critical [Zam86, see also section 4.2]. Indeed, the MonteCarlo data indicate a weak first-order transition for $J_{2}=0.6 J_{1}$ [KHFP08], $J_{2}=0.8 J_{1}$ and $J_{2}=0.9 J_{1}$ (figure 5.5) accompanied by large crossover scales. Thus, the system is not critical for these parameters and it is not surprising that this weak first-order transition is not detected by the transfer-matrix computations for cylinders with circumferences $L \leq 22$ and thus the resulting values for $c$ are not meaningful.

\section{\begin{tabular}{l|l} 
continuum limit & 5.2 .2
\end{tabular}}

Since the numerical methods are limited in the analysis of the phase transition for $J_{1} \approx J_{2}$ and not fully understood for $J_{1} / J_{2} \searrow 0$, the next step taken is to derive an equivalent continuum model where the concept of perturbative renormalization is applied.

The starting point is the conformally invariant fixed point $J_{1}=0$ (see figure 5.10) and a coupling $J_{1}$ between the two decoupled Ising models $A$ and $B$ is added perturbatively. In a first step, the spin variables on discrete lattice sites are replaced by continuous fields which resemble the underlying Néel order of the two decoupled antiferromagnetic Ising models. Thus, the staggered spin variables $S_{I}(I=A, B$ sublattice $)$ have to be transformed into smooth variables defined by

$$
\sigma_{I}(m, n) \propto(-1)^{m+n} S_{I}(m, n),
$$

where $(m, n)$ are the lattice coordinates. Note that the transformation in equation (5.4) is based on a specific choice of gauge but this does not affect the macroscopic properties of the system. The choice of the unit cell is shown in figure 5.10 and the coordinate system is rotated by an angle of $\pi / 4$; i.e., the axes point along the next-nearest neighbor bonds. For each unit cell nine sites enter the calculation of the Hamiltonian (see figure 5.10); of

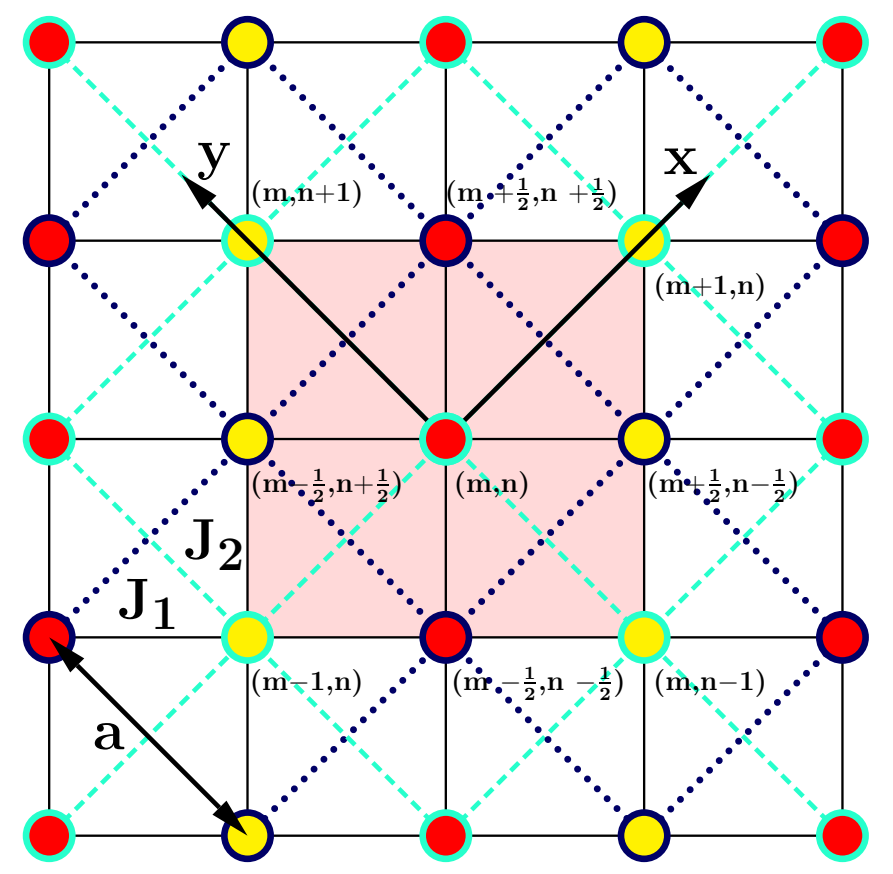

figure 5.10: (Published in [KHM11] and prepared by Marion Moliner.) Collinear phase of the $J_{1}-J_{2}$ square lattice. Red dots indicate up spins, and yellow dots indicate down spins. The two copies $A$ and $B$ of the Ising model with magnetic couplings $J_{2}$ and lattice spacing $a$ are, respectively, represented with dashed clear and dotted dark blue lines, while the black thin lines correspond to the $J_{1}$ square lattice. The shaded area represents the unit cell used to derive the continuum limit (section 5.2.2). The coordinates are indicated with respect to the $x$ and $y$ axis of the $A$ sublattice. 
these five $A$ sites have weights $1+4 \frac{1}{4}=2$ and four $B$ sites have weights $4 \frac{1}{2}=2$. Hence, an overall factor of 1/4 must be introduced. The Hamiltonian can then be evaluated per unit cell:

$$
\begin{gathered}
h_{A}=-\frac{J_{2}}{4} \sigma_{A}(x, y)\left[\sigma_{A}(x+1, y)+\sigma_{A}(x-1, y)\right. \\
\left.+\sigma_{A}(x, y+1)+\sigma_{A}(x, y-1)\right] \\
h_{B}=-\frac{J_{2}}{4}\left\{\sigma_{B}\left(x+\frac{1}{2}, y+\frac{1}{2}\right)\left[\sigma_{B}\left(x+\frac{1}{2}, y-\frac{1}{2}\right)+\sigma_{B}\left(x-\frac{1}{2}, y+\frac{1}{2}\right)\right]\right. \\
\left.+\sigma_{B}\left(x-\frac{1}{2}, y-\frac{1}{2}\right)\left[\sigma_{B}\left(x+\frac{1}{2}, y-\frac{1}{2}\right)+\sigma_{B}\left(x-\frac{1}{2}, y+\frac{1}{2}\right)\right]\right\} \\
h_{A B}=\frac{J_{1}}{4}\left\{\begin{array}{c}
\sigma_{A}(x, y)\left[\sigma_{B}\left(x+\frac{1}{2}, y+\frac{1}{2}\right)+\sigma_{B}\left(x-\frac{1}{2}, y-\frac{1}{2}\right)\right. \\
\left.-\sigma_{B}\left(x-\frac{1}{2}, y+\frac{1}{2}\right)-\sigma_{B}\left(x+\frac{1}{2}, y-\frac{1}{2}\right)\right] \\
-\frac{1}{2} \sigma_{B}\left(x+\frac{1}{2}, y+\frac{1}{2}\right)\left[\sigma_{A}(x+1, y)+\sigma_{A}(x, y+1)\right] \\
-\frac{1}{2} \sigma_{B}\left(x-\frac{1}{2}, y-\frac{1}{2}\right)\left[\sigma_{A}(x-1, y)+\sigma_{A}(x, y-1)\right] \\
+\frac{1}{2} \sigma_{B}\left(x+\frac{1}{2}, y-\frac{1}{2}\right)\left[\sigma_{A}(x+1, y)+\sigma_{A}(x, y-1)\right] \\
\left.+\frac{1}{2} \sigma_{B}\left(x-\frac{1}{2}, y+\frac{1}{2}\right)\left[\sigma_{A}(x-1, y)+\sigma_{A}(x, y+1)\right]\right\} .
\end{array}\right.
\end{gathered}
$$

A Taylor expansion up to second order is applied on the $\sigma_{I}=\sigma_{I}(x, y)$ fields around the unit-cell center:

$$
\begin{aligned}
\sigma_{I}(x+m a, y+n a)= & \sigma_{I}+a\left[m \partial_{x}+n \partial_{y}\right] \sigma_{I} \\
& +\frac{a^{2}}{2}\left[m^{2} \partial_{x x}^{2}+n^{2} \partial_{y y}^{2}+2 m n \partial_{x y}^{2}\right] \sigma_{I}+\mathcal{O}\left(\partial^{3} \sigma_{I}\right),
\end{aligned}
$$

where $a$ represents the lattice spacing. Expanding all products in equation (5.7) up to second order leaves only two terms (a detailed derivation can be found in appendix B.1):

$$
h_{A B}=-\frac{J_{1}}{4} a^{2}\left[\partial_{x} \sigma_{A} \partial_{y} \sigma_{B}+\partial_{y} \sigma_{A} \partial_{x} \sigma_{B}\right] .
$$

Note that partial derivatives are understood to act only on the subsequent operator. In particular all terms of zeroth order $\left(\sigma_{A} \sigma_{B}\right)$ and first order (e.g., $\left.\sigma_{A} \partial_{x} \sigma_{B}\right)$ are canceled out due to the symmetry of the underlying ground state. ${ }^{1}$ These symmetry arguments will also be discussed later for higher order terms. The pure models $h_{I}$ in equations (5.5) and (5.6) yield in zeroth order the expected spin-spin coupling (see appendix B.1 for details):

$$
h_{I}=-\frac{J_{2}}{4}\left[4 \sigma_{I} \sigma_{I}+a^{2} \sigma_{I} \partial_{x x} \sigma_{I}+a^{2} \sigma_{I} \partial_{x x} \sigma_{I}\right] .
$$

These terms represent the unperturbed model and its conformal theory and phase transition behavior is known. The focus is now on the perturbation introduced by $h_{A B}$ given in equation (5.9).

In a next step the sum of the Hamiltonian (5.1) is converted into a two-dimensional integral where the values of $\sigma_{I}(x, y)$ at the limits $( \pm \infty, \pm \infty)$ are equal because of the periodic boundary conditions imposed on the model:

$$
\sum_{m, n} \rightarrow \frac{1}{a^{2}} \int_{-\infty}^{\infty} d x d y
$$

\footnotetext{
${ }^{1}$ As mentioned above the calculations rely on a specific gauge of the unit cell. However, all calculations were also performed for a symmetric choice of the unit cell and yielded the same results. This is also discussed briefly in appendix B.1.
} 
For further calculations it is convenient to rewrite the interaction in equation (5.9) in complex coordinates $z=x+i y$ and $\bar{z}=x-i y$ (see appendix B.1):

$$
\begin{aligned}
H_{A B} & =-i \mu^{2} J_{1} \iint d z d \bar{z}\left(\mathcal{O}_{1}(z, \bar{z})-\mathcal{O}_{2}(z, \bar{z})\right) \text { with } \mu=\frac{1}{2} \\
\mathcal{O}_{1}(z, \bar{z}) & =\partial_{z} \sigma_{A} \partial_{z} \sigma_{B}, \mathcal{O}_{2}(z, \bar{z})=\partial_{\bar{z}} \sigma_{A} \partial_{\bar{z}} \sigma_{B} .
\end{aligned}
$$

As described in section 4.2 the $\sigma_{I}$ fields of the theory have conformal dimensions $\left(h_{\sigma}, \bar{h}_{\sigma}\right)=$ $(1 / 16,1 / 16)$, scaling dimension $\Delta=h+\bar{h}=1 / 8$, and conformal spin $s=h-\bar{h}=0$. The full model is then described by the action

$$
\begin{aligned}
& \mathcal{A}=\mathcal{A}_{A}^{0}+\mathcal{A}_{B}^{0}+\tau \iint d z d \bar{z}\left(\varepsilon_{A}(z, \bar{z})+\varepsilon_{B}(z, \bar{z})\right) \\
& +g \iint d z d \bar{z}\left(\mathcal{O}_{1}(z, \bar{z})-\mathcal{O}_{2}(z, \bar{z})\right), \quad g \propto J_{1},
\end{aligned}
$$

where $\mathcal{A}_{I}^{0}$ are the fixed-point actions of the Ising models $A$ and $B$. Furthermore $\varepsilon_{I}$ is the usual thermal operator of the conformal field theory on the two-dimensional Ising model with $\left(h_{\varepsilon}, \bar{h}_{\varepsilon}\right)=(1 / 2,1 / 2)$ and the corresponding mass $\tau \propto\left(T-T_{C}^{J_{1}=0}\right)$ [dFMS97, Mus10, see also section 4.2 .4$]$.

The operators $\mathcal{O}_{1}$ and $\mathcal{O}_{2}$ are kept, despite being irrelevant with a scaling dimension $\Delta=9 / 4$, due to their non-zero conformal spins $s= \pm 2$. The presence of such chiral terms was previously reported in other frustrated systems such as the anisotropic square lattice [SB04], the checkerboard [SFB05] and the Kagomé [SSB08] lattices. These twist terms [NGE98] are known to be likely to generate relevant or marginally relevant terms at higher-order expansions [AAL01, Tsv01].

\section{symmetries}

Before calculating higher-orders terms of the perturbative interaction, it is valuable to discuss the underlying symmetries of the model and the consequences for the continuous field theory. The Hamiltonian (5.1) is invariant under translations by multiples of the lattice spacing $a$. Although in the continuous form the lattice spacing only represents a lower cutoff to the integrals, the symmetry is preserved for the continuous form of the field theory because the same periodic boundary conditions are imposed on the integrals. Furthermore, the model is symmetric under rotation of angles $\pi / 2$ and reflection on the lines $(x, y=0),(x=0, y),(x, y=x)$ and $(x, y=-x)$ (corresponding to the diagonals and the vertical and horizontal line through the origin in figure 5.10 residing on an $A$ site of the lattice). Note that the rotations and reflections are not independent. In addition the model is symmetric under the exchange of the two sublattices, which is equivalent to the translation by one lattice spacing of the original lattice.

The reflections are summarized in table 5.2. The $\sigma_{I}$ fields are completely symmetric in $z$ and $\bar{z}$ and the Hamiltonian integrates over the whole complex plane for both variables.

\begin{tabular}{c|c|c|c|c}
\hline $\operatorname{dir}$. & $(x, y)$ & $(z, \bar{z})$ & $\left(\partial_{z}, \partial_{\bar{z}}\right)$ & $\left(\sigma_{A}, \sigma_{B}\right)$ \\
\hline$\swarrow$ & $(-x, y)$ & $(-\bar{z},-z)$ & $\left(-\partial_{\bar{z}},-\partial_{z}\right)$ & $\left(\sigma_{A},-\sigma_{B}\right)$ \\
$\searrow$ & $(x,-y)$ & $(\bar{z}, z)$ & $\left(\partial_{\bar{z}}, \partial_{z}\right)$ & $\left(\sigma_{A},-\sigma_{B}\right)$ \\
$\downarrow$ & $(-y,-x)$ & $(-i \bar{z}, i z)$ & $\left(i \partial_{\bar{z}},-i \partial_{z}\right)$ & $\left(\sigma_{A}, \sigma_{B}\right)$ \\
$\leftarrow$ & $(y, x)$ & $(i \bar{z},-i z)$ & $\left(-i \partial_{\bar{z}}, i \partial_{z}\right)$ & $\left(\sigma_{A}, \sigma_{B}\right)$ \\
\hline
\end{tabular}

table 5.2: Symmetry operations for the reflections on the diagonal, vertical and horizontal lines [directions (dir.) are given as perpendicular arrows] of the unit cell presented in figure 5.10. 
Crucial points of these transformations are: the additional sign that occurs for the $B$ sublattice $(\swarrow$ and $\searrow$ ), and the rotation of the partial derivatives onto the imaginary axis $(\downarrow$ and $\leftarrow)$. Thus, operators that contribute to the continuous field theory either have to be quadratic in the sublattice fields or contain an asymmetric contribution of fields residing on different sublattices, like those in equation (5.9). Furthermore, the rotation of the partial derivatives onto the imaginary axis ensures that by symmetry only operators containing an even number of partial derivatives are allowed.

Following these symmetry arguments, the appearance of certain operators in higher orders can be discussed. A highly relevant $(\Delta=1 / 4)$ spin-spin coupling $\sigma_{A}(z, \bar{z}) \sigma_{B}(z, \bar{z})$ such as the one appearing in the two-layer Ising model [DM98, SRT00] is not allowed, whereas energy operators $\varepsilon_{I}$ and combinations $\varepsilon_{I} \varepsilon_{J}$ are allowed since they transform like products of spin fields on the same sublattice.

$$
\begin{array}{l|l}
\text { operator product expansion } & 5.2 .3
\end{array}
$$

The higher-order perturbation terms were calculated via the standard operator product expansion [BPZ84a, dFMS97, Mus10, see also section 4.2.3]. This operation allows for replacing, inside a correlation function, a product of two or more operators by a combination of scaling operators consistent with the underlying theory. This closes the renormalization-group equations in the operator algebra of the model before discarding irrelevant perturbations. For the two-dimensional Ising model, the field content of the product of two fields is encoded in the fusion rules given in equations (4.6)-(4.8). However only products of operators acting upon the same lattice yield non-zero results, because both lattices are independent at the starting fixed point:

$$
\begin{aligned}
{\left[\sigma_{I}\right]\left[\sigma_{J}\right] } & =\delta_{I, J}\left([\mathbb{1}]+\left[\varepsilon_{I}\right]\right) \\
{\left[\varepsilon_{I}\right]\left[\varepsilon_{J}\right] } & =\delta_{I, J}[\mathbb{1}] \\
{\left[\sigma_{I}\right]\left[\varepsilon_{J}\right] } & =\delta_{I, J}\left[\sigma_{I}\right] .
\end{aligned}
$$

Together with the general normalized form of the operator product expansion given in equation (4.9) one obtains

$$
\begin{aligned}
\sigma_{I}(z, \bar{z}) \sigma_{J}(w, \bar{w})= & \frac{\delta_{I, J}}{|z-w|^{1 / 4}}+\frac{\delta_{I, J}}{2}|z-w|^{3 / 4} \varepsilon_{I}(w, \bar{w}) \\
& +\frac{\delta_{I, J}}{4}(z-w)^{11 / 8}(\bar{z}-\bar{w})^{3 / 8} \partial_{w} \epsilon_{I}(w, \bar{w})+\text { H.c. }
\end{aligned}
$$

Note that marginal terms with non-zero conformal spin are kept consistent with previous comments about the relation of the eventual importance of chiral terms in frustrated systems.

By calculating the operator product expansion between the $\mathcal{O}_{1,2}$ operators of equation (5.13) one generates higher-order terms. At second order this generates terms $[\varepsilon=\varepsilon(w, \bar{w})]:$

$$
\begin{aligned}
& \propto \varepsilon_{I}, \quad \propto \partial_{\{w, \bar{w}\}} \varepsilon_{I}, \propto \varepsilon_{I} \varepsilon_{J} \\
& \propto \varepsilon_{I} \partial_{\{w, \bar{w}\}} \varepsilon_{J} \text { and } \propto \partial_{\{w, \bar{w}\}} \varepsilon_{I} \partial_{\{w, \bar{w}\}} \varepsilon_{J} \quad(I \neq J) .
\end{aligned}
$$


In detail, the calculations presented in appendix B.2.1 yield the following terms:

$$
\begin{aligned}
& {\left[\mathcal{O}_{1}(z, \bar{z})-\mathcal{O}_{2}(z, \bar{z})\right]\left[\mathcal{O}_{1}(w, \bar{w})-\mathcal{O}_{2}(w, \bar{w})\right]=} \\
& \alpha_{0}\left[(z-w)^{-\frac{17}{4}}(\bar{z}-\bar{w})^{-\frac{1}{4}}+\text { H.c. }\right]-\frac{2}{4096}|z-w|^{-\frac{9}{2}} \\
& -\left\{\alpha_{1}\left[(z-w)^{-\frac{15}{4}}(\bar{z}-\bar{w})^{\frac{1}{4}}+\text { H.c. }\right]-\frac{9}{4096}|z-w|^{-\frac{7}{2}}\right\}\left(\varepsilon_{A}+\varepsilon_{B}\right) \\
& +\left\{\alpha_{2}\left[(z-w)^{-\frac{13}{4}}(\bar{z}-\bar{w})^{\frac{3}{4}}+\text { H.c. }\right]-\frac{81}{8192}|z-w|^{-\frac{5}{2}}\right\}\left(\varepsilon_{A} \varepsilon_{B}\right) \\
& -\left(\alpha_{3}(z-w)^{-\frac{3}{4}}(\bar{z}-\bar{w})^{-\frac{7}{4}}+\alpha_{4}(z-w)^{-\frac{11}{4}}(\bar{z}-\bar{w})^{\frac{1}{4}}+\alpha_{3}(z-w)^{\frac{5}{4}}(\bar{z}-\bar{w})^{-\frac{15}{4}}\right) \times \\
& +\left(\partial_{w} \varepsilon_{A}+\partial_{w} \varepsilon_{B}\right)+\text { H.c. } \\
& \quad\left(\alpha_{5}(z-w)^{-\frac{9}{4}}(\bar{z}-\bar{w})^{\frac{3}{4}}-\alpha_{6}(z-w)^{-\frac{1}{4}}(\bar{z}-\bar{w})^{-\frac{5}{4}}+\alpha_{5}+\varepsilon_{A} \partial_{w} \varepsilon_{B}\right)+\text { H.c. } \\
& \left.+\sum_{k} \beta_{k}(z-w)^{v_{k}}(\bar{z}-\bar{w})^{t_{k}}\left(\partial_{w} \varepsilon_{A} \partial_{w} \varepsilon_{B}\right)\right)+ \text { H.c. }, \quad v_{k} \neq t_{k} .
\end{aligned}
$$

The coefficients $\alpha_{i}, \beta_{k}$ and exponents $v_{k}, t_{k}$ are rational constants, and the terms in equations (5.24) and (5.25) are irrelevant but could - as shown before - produce relevant terms in higher-order expansions.

Before computing third order terms it is important to discuss the structure of the prefactors $\propto(z-w)^{p} \cdot(\bar{z}-\bar{w})^{q}$ appearing in all equations above. After the integration of the prefactors (details presented in appendix B.2.2) only terms from equations (5.20), (5.21) and (5.22) are left that fulfill $p=q$ :

$$
-\frac{2}{4096}|z-w|^{-\frac{9}{2}}, \quad \frac{9}{4096}|z-w|^{-\frac{7}{2}}\left(\varepsilon_{A}+\varepsilon_{B}\right), \quad-\frac{81}{8192}|z-w|^{-\frac{5}{2}}\left(\varepsilon_{A} \varepsilon_{B}\right) .
$$

The third-order terms $\left(\propto J_{1}^{3}\right)$ are obtained by multiplying the above operators with the original perturbation given in equation (5.13). The following operator product expansion applies (see equations (4.10) and (5.16)):

$$
\begin{aligned}
\varepsilon_{J}(w, \bar{w}) \sigma_{I}(s, \bar{s}) & =\frac{\delta_{I, J}}{2}|w-s|^{-1} \sigma_{I}(s, \bar{s}) \\
+ & \frac{\delta_{I, J}}{4}(w-s)^{\frac{1}{2}}(\bar{w}-\bar{s})^{-\frac{1}{2}} \partial_{s} \sigma_{I}(s, \bar{s})+\text { H.c. }
\end{aligned}
$$

This yields only three different types of operators: these are primary and secondary operators from the spin family

$$
\begin{aligned}
\propto \sigma_{I} \sigma_{J}, & \propto \sigma_{I} \partial_{\{s, \bar{s}\}} \sigma_{J} \\
\text { and } & \propto \partial_{\{s, \bar{s}\}} \sigma_{I} \partial_{\{s, \bar{s}\}} \sigma_{J} .
\end{aligned}
$$

Calculating the third-order perturbation by multiplying every remaining operator from the second order [equation (5.26)] with $\left[\mathcal{O}_{1}-\mathcal{O}_{2}\right]$ yields the prefactors for these spin operators (derivation in appendix B.2.3). However, for each of the relevant operators in equation (5.28) the prefactors are canceled by the polar integration for $d w d \bar{w}$, which is in agreement with the symmetry considerations presented above. Thus, the third-order perturbation does not give any new operators since the irrelevant terms in equation (5.29) 
are the same as in the first order of the perturbation. Thus, the operator algebra is closed [dFMS97, chapter 6] and only the terms of equation (5.26), in particular the thermal operators $\varepsilon_{A}+\varepsilon_{B}$ and the marginal operator $\varepsilon_{A} \varepsilon_{B}$ are left:

$$
\begin{aligned}
& -\frac{9 \mu^{4} J_{1}^{2}}{4096} \int_{|z-w|>a} d z d \bar{z} d w d \bar{w}|z-w|^{-\frac{7}{2}}\left(\varepsilon_{A}+\varepsilon_{B}\right) \\
& +\frac{81 \mu^{4} J_{1}^{2}}{8192} \int_{|z-w|>a} d z d \bar{z} d w d \bar{w}|z-w|^{-\frac{5}{2}} \varepsilon_{A} \varepsilon_{B} .
\end{aligned}
$$

The signs of these operators stem from the squares of the coupling given in equation (5.12) and the derived signs of the perturbative calculations given in equations (5.21) and (5.22), respectively. The pure energy terms in equation (5.30) and the mass term in equation (5.14) are necessary to tune the model onto criticality. The new critical temperature up to second order in perturbation theory is given by

$$
T_{C}\left(J_{1}\right)=T_{C}(0)-\gamma J_{1}^{2}
$$

which is in agreement with the behavior of the critical line on the left-hand side of the phase diagram presented in figure 5.3. The rescaled mass term is equal to zero on the critical line. Thus, the most relevant perturbation is the marginal energy-energy coupling [equation (5.31)]. Together with the action of the unperturbed Ising models from equation (5.14) the Ashkin-Teller field theory is readily identified [AT43, DM98, see also section 2.2.2]:

$$
\mathcal{A}_{A T}=\mathcal{A}_{A}^{0}+\mathcal{A}_{B}^{0}+k \int d w d \bar{w} \varepsilon_{A} \varepsilon_{B}
$$

where $k \propto J_{1}^{2}$ is the renormalized coupling constant. The sign of the marginal perturbation in principle can matter, but for the present case it does not affect the conclusion that one stays on the critical line. Since the free fermionic theory is located in the middle of a line of $c=1$ conformal field theories (see, for example, reference [Gin88, confer the vertical line in figure 14 on page 134]), the theory is critical on either side of the fixed point of two decoupled Ising models.

\section{Ashkin-Teller model $\quad 5.2 .4$}

The lattice model consists of two Ising models with internal coupling $J$ which are additionally coupled via a four-spin interaction $J_{4}$ (confer 2.2.2). The field-theoretic action of this model is the same as in equation (5.33) with a coupling constant $k=f\left(J, J_{4}\right)$. Thus, the four-spin interaction is mimicked by the perturbative onset of the energy-energy coupling, which is, in the present case, proportional to the square of the original nearestneighbor coupling $J_{1}$. The rich phase diagram of the Ashkin-Teller model given in coupling constants $J$ and $J_{4}$ in references [Bax82, DG04] includes a critical line which represents the one-dimensional flow diagram of the corresponding field theory [equation (5.33)] at criticality with a single parameter given by the renormalized coupling $k$. It starts for $k=0$ at the conformally invariant fixed point of two decoupled Ising models and ends at a Potts-critical end point. The presented calculations yield a conformal field theory with $c=1$ in the presence of a marginal operator. Both are necessary conditions to fulfill 
so that the theory can exhibit varying critical exponents [FQS84, KW71]. This scenario would be consistent with the numerical results for large $J_{2}$ and earlier descriptions of the frustrated Ising model [LB05]. The Potts-critical end point would also allow for an onset of a non-critical line of first-order phase transitions as observed in the Monte-Carlo simulations.

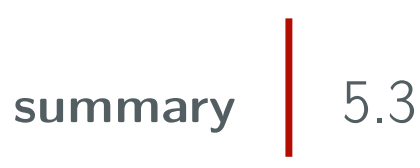

The frustrated $J_{1}-J_{2}$ Ising model on the square lattice was investigated using classical Monte-Carlo simulations, transfer-matrix calculations and field-theoretic methods known from conformal field theory. The focus was on the analysis of the finite-temperature phase transitions, in particular from a collinear ordered state into the paramagnetic state. The phase-transition scenario obtained in this chapter is sketched in figure 5.11.

The evaluation of large-scale histograms indicates a first-order transition for parameters $0.5 J_{1}<J_{2} \lesssim 0.9 J_{1}$, but correlation functions prove a strong decoupling of the two sublattices of the square lattice model for $J_{2} \geq J_{1}$ and hint towards continuous phase transitions in this region of the phase diagram. In-between $\left(J_{2} \approx J_{1}\right)$ a more detailed analysis is hampered by large crossover scales and reliable results cannot be obtained from the finite-size lattice simulations.

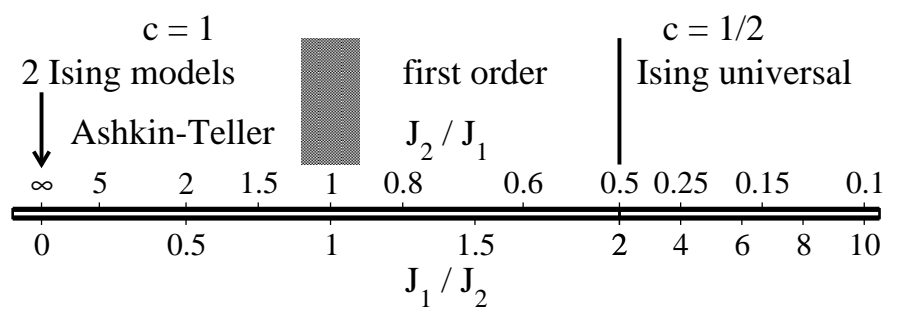

figure 5.11: Phase transition scenario for the frustrated $J_{1^{-}} J_{2}$ Ising model. The transition into the Néel phase for $J_{1}>2 J_{2}$ is Ising universal whereas the transition into the collinear phase shows first-order behavior for $2 J_{2}>J_{1} \gtrsim J_{2}$ and continuous but non-universal behavior for smaller $J_{1}$ which is explained by the underlying Ashkin-Teller field theory.

Because the critical two-dimensional model can be mapped onto a quantum field theory which is characterized by a central charge $c$, transfer-matrix calculations were performed to derive the central charge of the underlying field theory. The results for $J_{1} / J_{2} \searrow 0$ are consistent with $c=1$ as expected for two decoupled Ising models. However, for $J_{1} / J_{2} \nearrow 1$ the findings are not consistent and probably suffer the same finite-size problems as the Monte-Carlo simulations.

For this reason the continuum limit of the model was derived and the coupling of the two sublattices was investigated perturbativly using operator product expansions. As a result, in second-order perturbation theory relevant energy terms rescale the transition temperature of the decoupled case $\left(J_{1}=0\right)$ towards lower values and a marginal energyenergy coupling arises which is also known as Ashkin-Teller term. Thus, a critical model with $c=1$ is recovered, which together with the marginal coupling allows for non-universal critical exponents. 


\section{incommensurate ordering in a spatially anisotropic Ising model}

This project was initiated by Gennady Chitov from the Laurentian University in Sudbury (Canada) who proposed to calculate correlation functions for a spatially anisotropic version of the $J_{1}-J_{2}$ Ising model. In this chapter Monte-Carlo results for this model are reported that are not yet published.

\section{abstract}

The anisotropic version of the $J_{1^{-}} J_{2}^{a, b}$ Ising model is investigated by means of MonteCarlo simulations. In particular the ordering process from the paramagnetic into an antiferromagnetic phase with diagonal ferromagnetic stripes (ordering wave vector $\mathbf{q}=$ $(\pi, 2, \pi / 2))$ is analyzed, and the finite-temperature behavior of energies, specific heats and correlation functions is presented. A comparison of structure factors for different lattice sizes reveals an incommensurate ordering for intermediate temperatures.

The model of interest in this chapter is given by a specific choice of the square lattice Ising model presented in equation (2.9):

$$
H_{\text {Ising, aniso. }}=J_{1} \sum_{\mathrm{NN}} S_{i} S_{j}+J_{2} \sum_{\mathrm{NNN}, /} S_{i} S_{j}-J_{2} \sum_{\mathrm{NNN}, \backslash} S_{i} S_{j}
$$

where $/$ and \ indicate the two perpendicular next-nearest neighbor bonds and the opposite sign for these couplings distinguishes the present model from the isotropic version discussed in the previous chapter. Three different ground states are readily identified: a ferromagnetic state or Néel ordered state for dominating nearest-neighbor interactions $\left|J_{1}\right|>\left|J_{2}\right|$ and a superferromagnetic-antiferromagnetic (SFAF) state for $\left|J_{1}\right|<\left|J_{2}\right|$; all three states are sketched in figure 6.1. The phase transitions to the ferromagnetic and Néel ordered state are continuous and belong to the Ising universality class. However, for the remaining finite-temperature phase transition into the SFAF state the analysis is more complicated. Chitov and Gros performed mean-field calculations [CG05] and proposed a scenario that includes an onset of incommensurate ordering before the system is thermalized into its ground state. However, it is known that mean-field calculations

$\begin{array}{llllllllll} & \text { FO } & & & \text { SFAF } & & \text { NO } & \text { figure } 6.1 \text { : } & \text { Three } \\ \text { different ground state con- } \\ \text { figurations: Néel order (NO) }\end{array}$




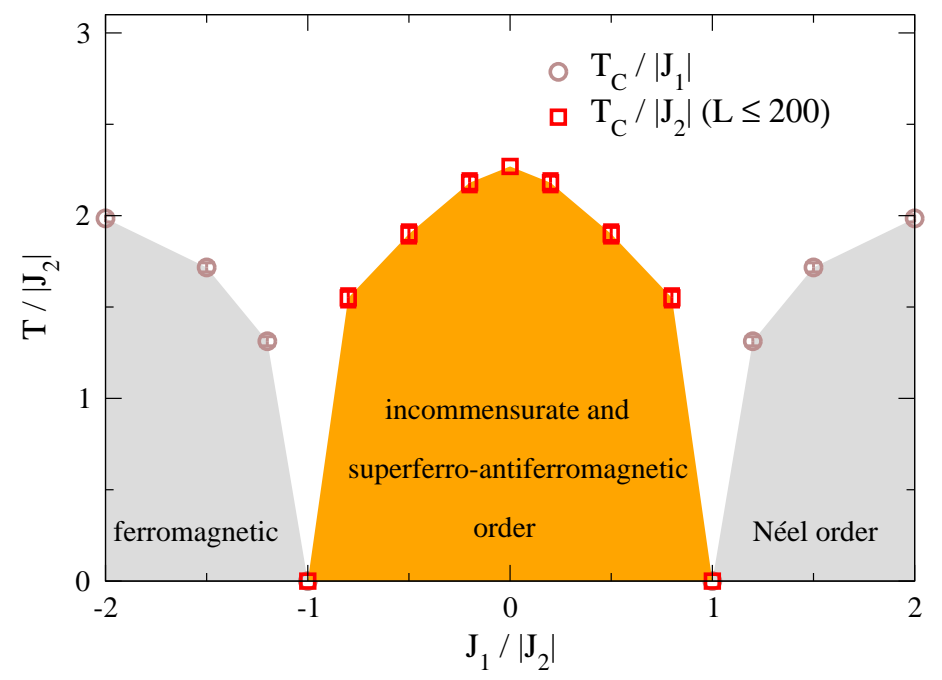

figure 6.2: $\quad$ Phase diagram of the anisotropic Ising model for varying nearest-neighbor interactions $J_{1} /\left|J_{2}\right|$. Critical temperatures are determined using Binder cumulants for $\left|J_{1}\right|>\left|J_{2}\right|$ and estimated from energies and specific heats for $\left|J_{1}\right|<\left|J_{2}\right|$, and strongly depend on the system size - here $L \leq 200$ (see text for more details). The agreement with a qualitative sketch in [CG05] is very good.

in low-dimensional systems $(d<4)$ are limited in the description of phase transitions [Gol93] and, hence, the main task of the following chapter is to check their prediction by a complementary method. For this purpose, Monte-Carlo simulations are preformed and the ordering process is evaluated by calculating correlation functions for different system sizes and analyzing their Fourier transform - the structure factor for particular wave vectors $\mathbf{q}=\left(q_{x}, q_{x}\right)$.

\section{\begin{tabular}{l|l} 
phase diagram & 6.1
\end{tabular}}

The phase diagram for the model was introduced in reference [CG05] for varying parameters $J_{1}, J_{2}^{a}$ and $J_{2}^{b}$. The result is a three-dimensional qualitative phase diagram including ferromagnetic and various antiferromagnetic phases (figure 2 of [CG05]). In the present work the focus lies on a one-dimensional cut through this phase diagram with a varying nearest-neighbor coupling $J_{1}$ and a fixed value $J_{2}^{a}=-J_{2}^{b}$. The finite-temperature phase diagram $T_{C}-J_{1} /\left|J_{2}\right|$ was qualitatively sketched in figure 6 of reference [CG05, mean-field calculations] and the equivalent is presented here in figure 6.2 by means of Monte-Carlo simulations and the qualitative agreement is very good. Note that the transition temperatures are invariant under the change of the sign $J_{1} \rightarrow-J_{1}$; this was double-checked in particular for $\left|J_{1}\right|<\left|J_{2}\right|$. For the interactions $\left|J_{1}\right|>\left|J_{2}\right|$ the critical temperatures were determined using the Binder cumulants for different lattice sizes as for the isotropic model. On the other hand for $\left|J_{1}\right|<\left|J_{2}\right|$, the extraction of critical temperatures is more complicated since the order parameter for the SFAF state and its Binder cumulants show a strong finite-size dependence. Thus, the values in figure 6.2 are estimated by an analysis of the energy and specific heat. Furthermore, at this point no distinction between the SFAF and incommensurate ordering temperatures is made for the same reasons.

\section{energy and specific heat $\mid 6.2$}

The behavior of the energy already indicates that the ordering processes for the two phase transitions differ significantly. In figure 6.3 the temperature dependence of the energy is shown for three cases (a)-(c) with $J_{1}<\left|J_{2}\right|$ and compared to the energy (d) of an Ising 


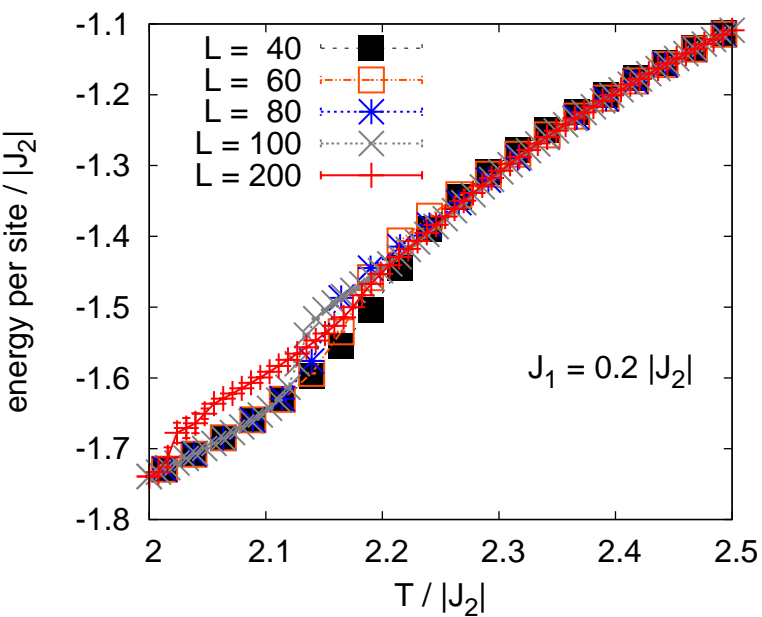

(a) $J_{1}=0.2\left|J_{2}\right|$, SFAF order for $T \searrow 0$

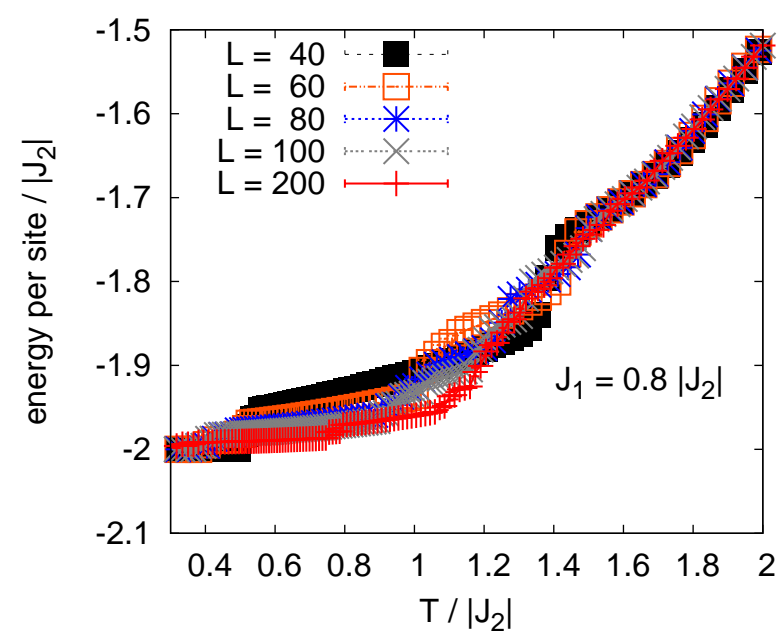

(c) $J_{1}=0.8\left|J_{2}\right|$, SFAF order for $T \searrow 0$

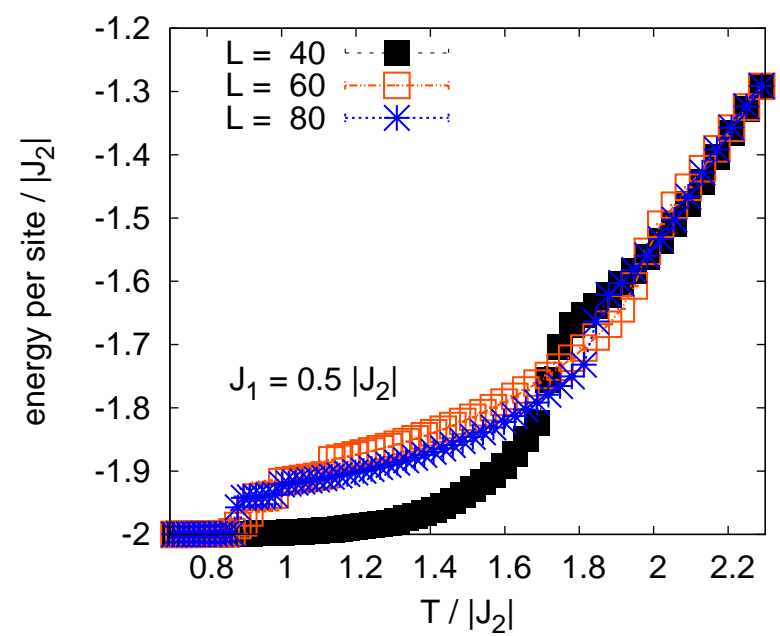

(b) $J_{1}=0.5\left|J_{2}\right|$, SFAF order for $T \searrow 0$

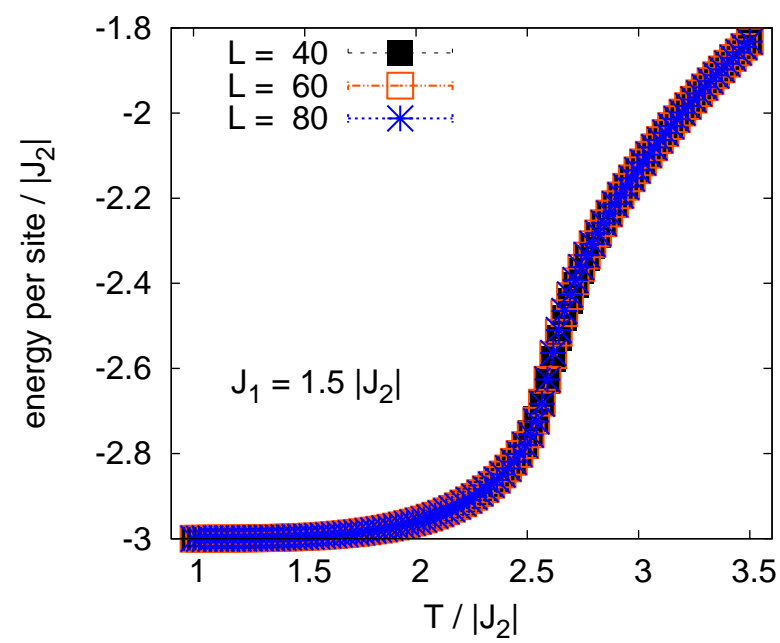

(d) $J_{1}=1.5\left|J_{2}\right|$, Néel order

figure 6.3: Energies for some values of nearest-neighbor coupling $J_{1}>0$. In figures (a) - (c) multiple steps appear which differ for different system sizes before reaching the groundstate energy $E_{\mathrm{SFAF}}=-2\left|J_{2}\right|$. As a comparison a converged energy development for the phase transition into the Néel ordered state is shown in (d) $-E_{\text {Néel }}=-2 J_{1}$.

phase transition at $J_{1}=1.5\left|J_{2}\right|$. The multiple steps in the energies, which in addition are shifted for different system sizes $L$, hint towards an unusual ordering in the model that involves different size-dependent intermediate states. This strong size dependence already hints towards incommensurate ordering.

Because such phase transitions are also accompanied by large energy fluctuations, an investigation of the specific heat (defined in section 2.1) could yield more insight. In figure 6.4 specific heats for increasing systems are compared for $J_{1}=0.2\left|J_{2}\right|$ and $J_{1}=1.5\left|J_{2}\right|$. In figure $6.4 \mathrm{a}$ one can distinguish different peaks arising for larger lattices $L \geq 80$ for the phase transition into the SFAF phase. This emphasizes that indeed not only one phase transition occurs but several. Therefore, a set of different intermediate states - the number depends on the system size - are present in the anisotropic Ising model on a finite lattice. The behavior of a normal continuous phase transition is recovered in the specific heat at $J_{1}=1.5\left|J_{2}\right|$ as expected for the transition into the Néel phase. 


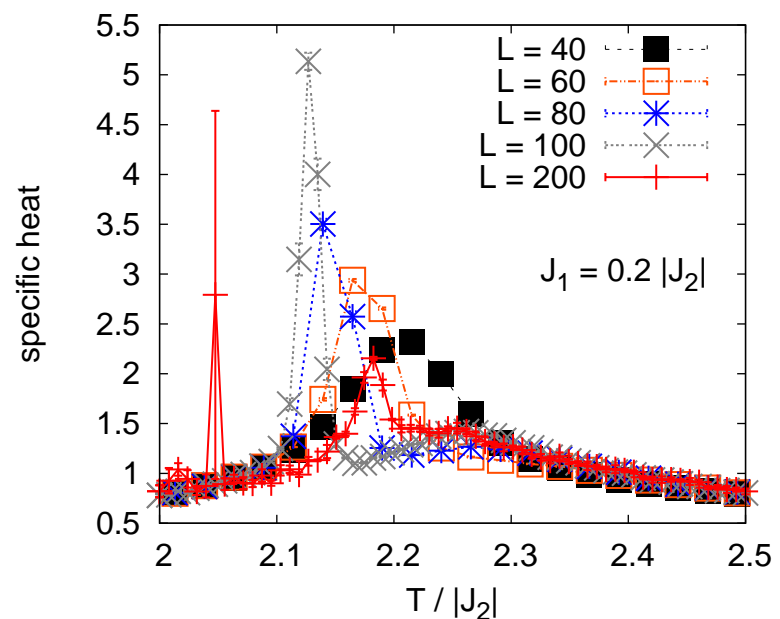

(a) $J_{1}=0.2\left|J_{2}\right|$, SFAF order for $T \searrow 0$

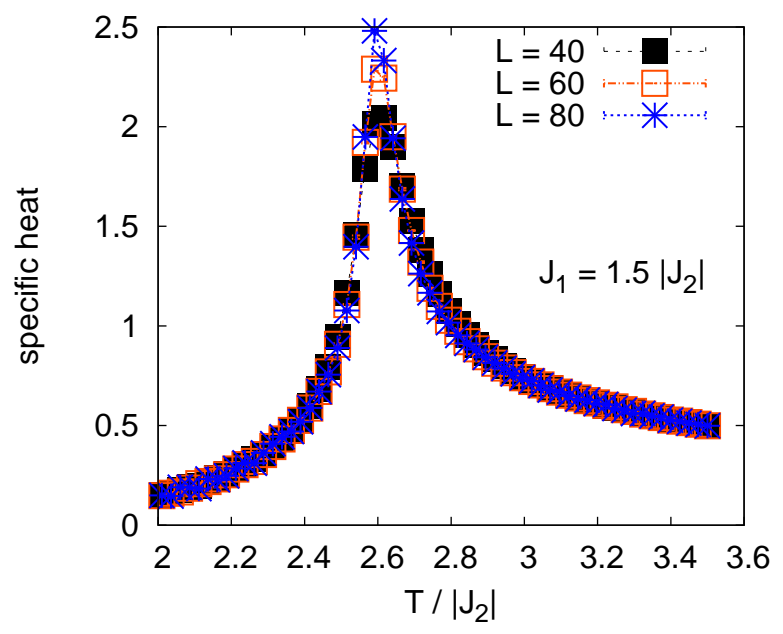

(b) $J_{1}=1.5\left|J_{2}\right|$, Néel order

figure 6.4: Specific heats for different system sizes. (a) As already indicated by the energy, multiple peaks are distinguished the specific heat for increasing system sizes. (b) The position of the peak is nearly fixed and the height scales moderately with the system size as expected for a continuous phase transition.

For the characterization of these various phase transitions energy histograms are recorded. In figure 6.5 two sets of histograms are shown that clearly prove the firstorder nature of the emerging transitions. On the left-hand side histograms for the ultimate phase transition into the ground state (SFAF state) are shown at $J_{1}=0.2\left|J_{2}\right|$ for $L=100,200$, i.e., the temperatures at which the histograms are recorded correspond to the last step and peak in the energies and specific heats respectively. Although the transition temperature is shifted to a lower value, the double-peaked structure becomes more pronounced. Furthermore, the scaling of peak-to-peak distance is consistent with

$$
\begin{array}{r}
L=100, T=2.128 /\left|J_{2}\right| \\
L=200, T=2.0176 /\left|J_{2}\right|
\end{array}
$$

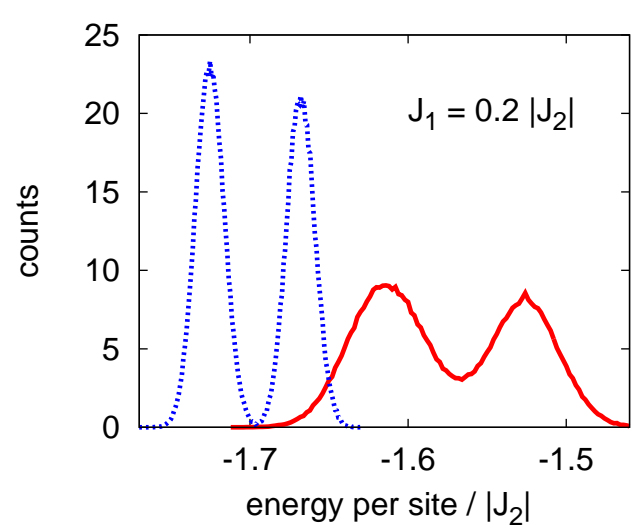

(a) $J_{1}=0.2\left|J_{2}\right|$, SFAF transition

$$
\begin{aligned}
& T=1.17444 /\left|\mathrm{J}_{2}\right|- \\
& T=1.18978 /\left|\mathrm{J}_{2}\right| \ldots . . . . . . . . . . . \\
& T=1.20533 /\left|\mathrm{J}_{2}\right|
\end{aligned}
$$$$
\begin{aligned}
& T=1.30299 /\left|\mathrm{J}_{2}\right| \cdots \\
& T=1.32002 /\left|\mathrm{J}_{2}\right|-\cdots \\
& T=1.33727 /\left|\mathrm{J}_{2}\right|
\end{aligned}
$$

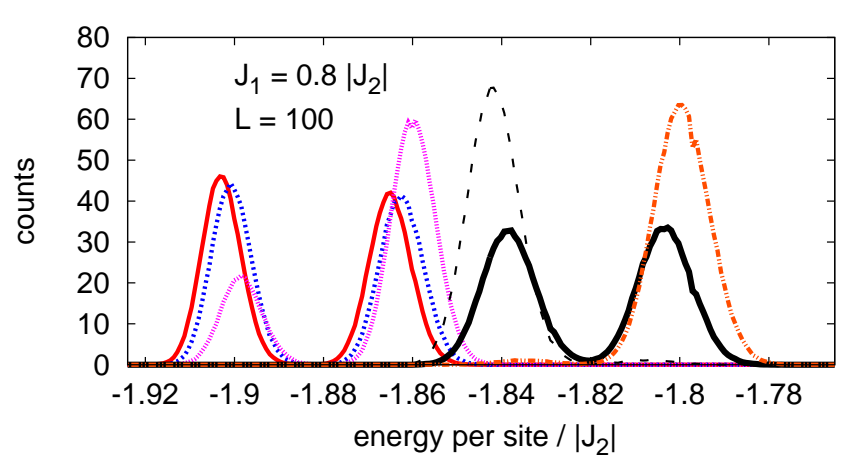

(b) $J_{1}=0.8\left|J_{2}\right|, L=100$, intermediate transitions

figure 6.5: Normalized histograms for some phase transitions in the anisotropic Ising model that clearly prove the first-order character of the transitions. (a) Phase transitions into the SFAF ground state; for larger lattices the transition temperature is shifted to a lower value. (b) Two different phase transitions - both of first order - can be distinguished. In the middle some nearly Gaussian shaped histograms were left out for clarity. 
the inverse lattice sizes. On the right-hand side, i.e., in figure $6.5 \mathrm{~b}$ various histograms at $J_{1}=0.8\left|J_{2}\right|$ and for $L=100$ are plotted. Two transitions are separated only by a small energy range and both show doubly-peaked histograms. Thus, the intermediate states on the finite lattice are also separated by first-order phase transitions, as was expected by the step-like development of the energy.

\section{\begin{tabular}{l|l} 
order parameter and correlation functions & 6.3
\end{tabular}}

To gain further insight into the ordering process of the system it is useful to define order parameters and analyze their behavior around the phase transition. The wave vector of the SFAF phase is given by $\mathbf{q}=(\pi / 2, \pi / 2)$ because the unit cell is a $4 \times 4$ state (confer figure 6.1 middle). Hence, the square root of the normalized structure factor at this wave vector yields a good order parameter. The calculation of this order parameter can be implemented using a staggered magnetization

$$
\begin{aligned}
& m_{\mathrm{SFAF}, k}=\sqrt{\frac{S(\pi / 2, \pi / 2)}{N}}=\frac{1}{N} \sum_{i}(-1)^{f_{k}\left(r_{i}^{x} \cdot r_{i}^{y}\right)} S_{i} \\
& f_{0}\left(r_{x}, r_{y}\right)=\left[\left(r_{x}+r_{y}\right) / 2\right] \% 2 \wedge f_{1}\left(r_{x}, r_{y}\right)=\left[\left(r_{x}+r_{y}+1\right) / 2\right] \% 2
\end{aligned}
$$

The modulo operation ' $\% 2$ ' yields values zero and one, and the two versions $f_{0,1}$ account for the degeneracy of the ground state, i.e., a shift of all spins by one lattice spacing. In addition both states can be flipped completely. In figure 6.6 this order parameter and its Binder cumulant are shown for increasing lattice sizes and a small nearest-neighbor coupling $J_{1}=0.2\left|J_{2}\right|$. The order parameter reflects the same behavior as observed in the energies, i.e., the onset of a non-zero signal is size-dependent. Even more instructive is the behavior of the Binder cumulant; already for small lattices $L \leq 100$, for which the order parameter seems to converge towards a fixed transition temperature, the cumulants

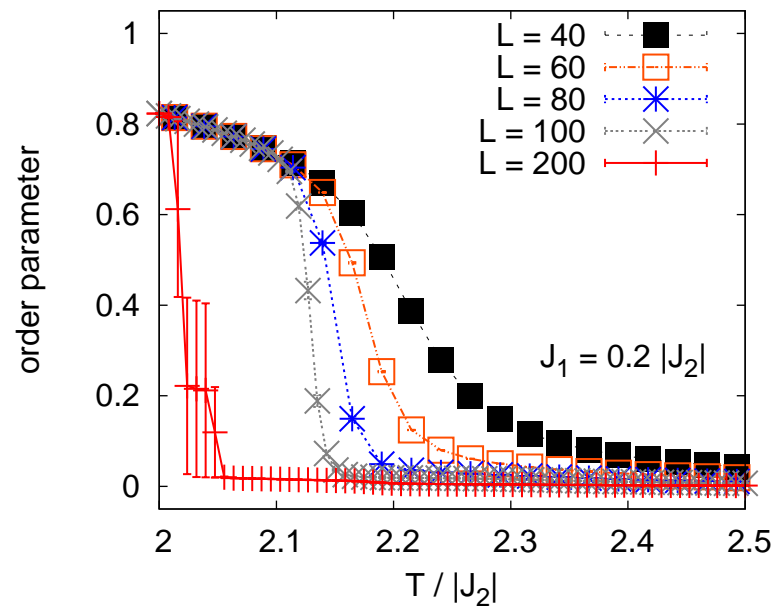

(a) order parameter

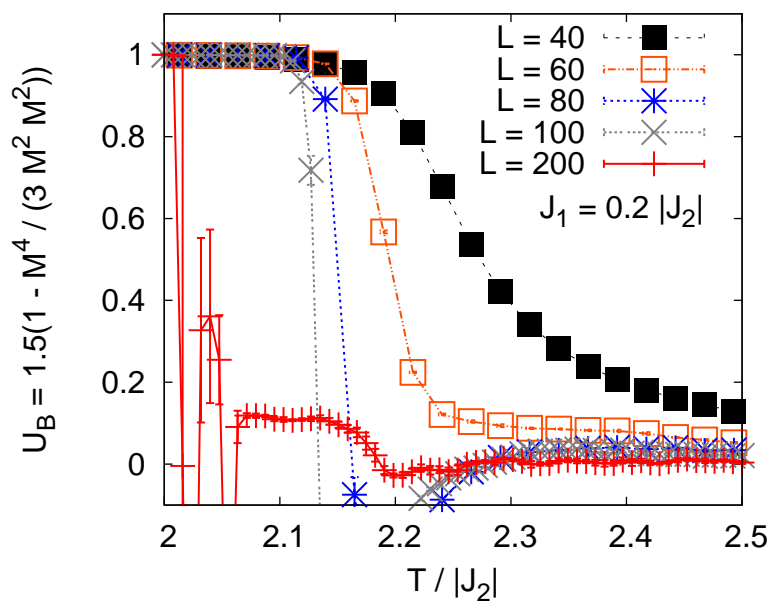

(b) Binder cumulants

figure 6.6: (a) The onset of the order parameter becomes more pronounced for larger lattices and is shifted to lower temperatures. (b) Because the transition temperature depends strongly on the system size the cumulants do not intersect in a single point. The large errors are caused by the abrupt transitions which are not well captured by the Monte-Carlo simulations. 


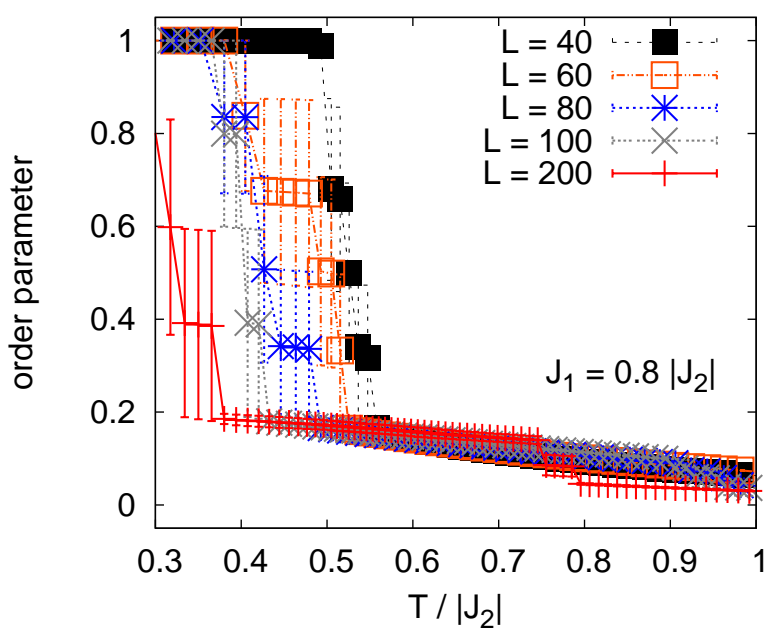

(a) order parameters

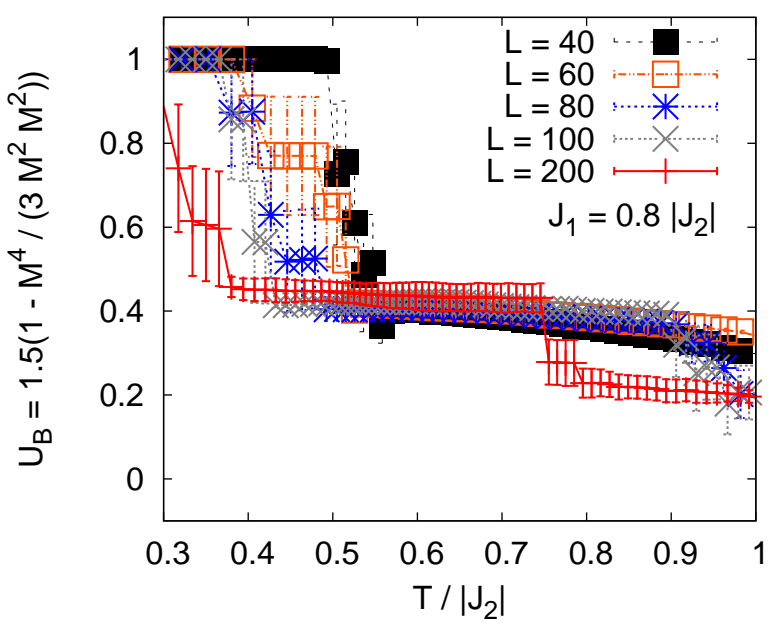

(b) Binder cumulants

figure 6.7: For every lattice size various transitions are revealed at different temperatures in the order parameter as well as in the cumulants. The large error bars are due to the sharp transitions.

do not intersect, and at lower temperatures for the largest system $(L=200)$ different steps appear in the cumulant. The abrupt transitions in the large system makes a good statistic evaluation of the Monte-Carlo data complicated and causes large errors. However, it is evident that the transition temperature is not universal. For larger values of $J_{1}$ the ordering process becomes more complicated. In figure 6.7 various steps are identified in the evolution of the order parameter and the Binder cumulant, although both plots are dominated by the large error bars that stem again from the statistical analysis of the sharp transitions.

\section{correlation functions}

The correlation functions $S_{i} S_{j}$ are measured along rows and columns of the square lattice, i.e., $i_{y}=j_{y}$ or $i_{x}=j_{x}$. Since $S_{i}= \pm 1$, they can adopt values between -1 and +1 . However, to demonstrate the modulation of the amplitude in dependence of the distance $r=i_{m}-j_{m}$ it is more convenient to plot the absolute value of the correlation functions. Furthermore, like in the previous chapter, a separate illustration for even and odd distances $r$ is given. In figure 6.8 the amplitudes of the correlations are plotted with a color code for a finite temperature and lattice interval for a very small $\left(L=20, r_{\max }=9\right)$ and a larger lattice $\left(L=80, r_{\text {max. }}=39\right)$ both at $J_{1}=-0.8\left|J_{2}\right|$. The maximum distance is limited to $L / 2$ due to the periodic boundary conditions. In the small system only two amplitude modulations can be clearly distinguished: for high temperatures two peaks and for intermediate temperatures $\left(T \approx 1.5\left|J_{2}\right|\right)$ a single minimum at a medium even distance $r$ is obtained (where correlations at odd distances are enhanced). Both amplitude modulations are marked by black horizontal lines. In addition the SFAF state yields constant correlations for even distances at low temperatures. For the larger system (figure 6.8b, $L=80$ ) several modulations are visible that are divided by sharp transitions. Indeed, five different patterns are identified with a decreasing number of extrema (indicated by black horizontal lines). The ground state is classified in both cases by constant maximal correlations for even distances and zero correlations between neighboring sites. Thus, 

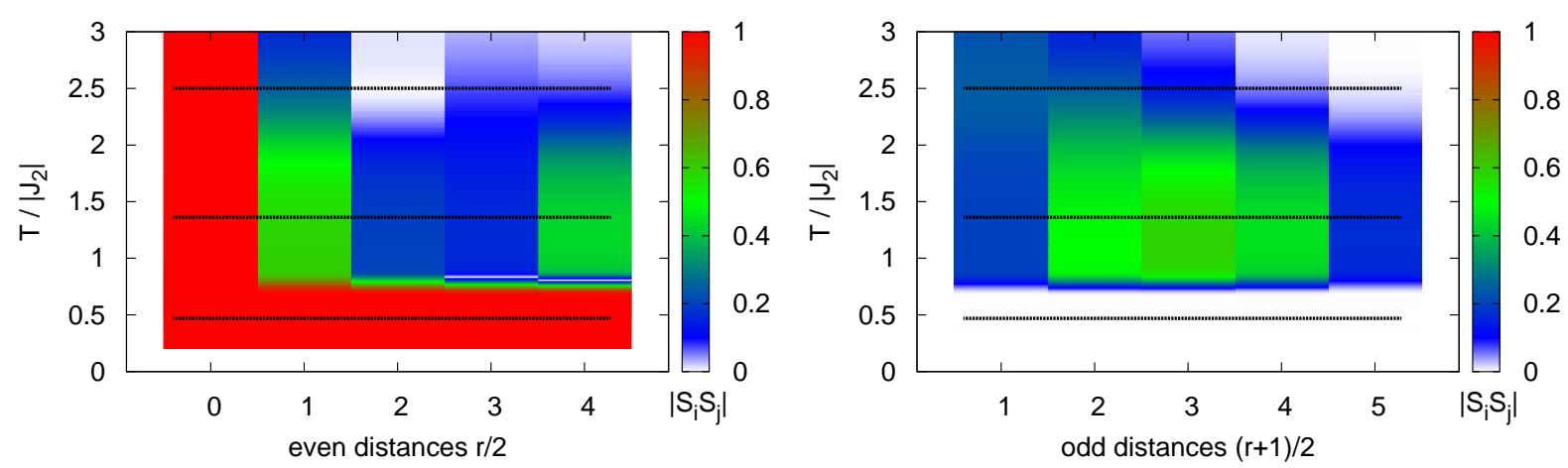

(a) $L=20, J_{1}=-0.8\left|J_{2}\right|$
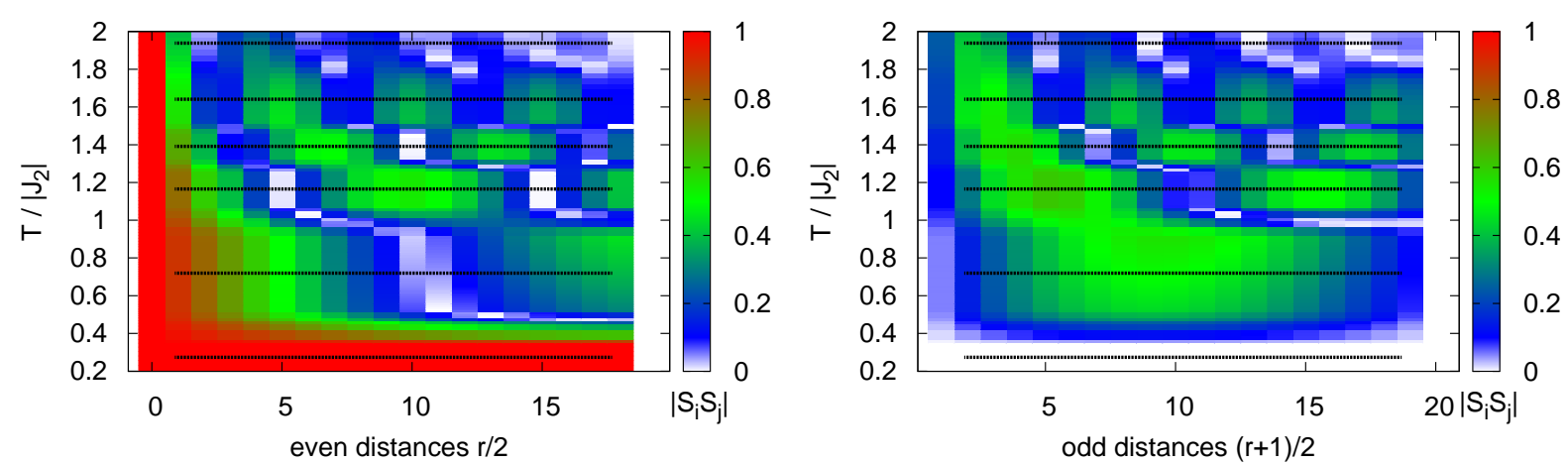

(b) $L=80, J_{1}=-0.8\left|J_{2}\right|$

figure 6.8: Absolute values of correlation functions $S_{i} S_{j}$ for even and odd distances $r=$ $i_{m}-j_{m}$ (the other lattice coordinate is fixed) and different temperatures. The modulation of the amplitude is clearly visible and for the larger lattice (b) a sequence of distinct wave vectors for the modulation are identified. Black lines indicate prominent stable patterns.

the two sublattices of the original square lattice are decoupled and both sublattices are ordered in a collinear antiferromagnetic state which yields the SFAF state in the original lattice.

\section{structure factors}

By analyzing the correlation functions it is obvious that a mixed phase of incommensurate and commensurate ordering is present for intermediate temperatures (above the ground state) which causes the development of superstructures in the correlation amplitude. To identity the exact wave vectors a Fourier analysis is necessary. However, according to Chitov and Gros [CG05, see, e.g., figure 5] the wave vectors of this incommensurate order lie on the very same line $\mathbf{q}=\left(q_{x}, q_{x}\right)$ as the already known vectors: ferromagnetic order $\mathbf{q}=(0,0)$, SFAF order $(\pi / 2, \pi / 2)$ and Néel order $(\pi, \pi)$. Moreover they find from their mean-field calculations that the incommensurate order is characterized by a single wave vector [CG05, page 726]

$$
q_{x}^{a}=q_{y}^{a}=\arccos \left(-\frac{J_{1}}{2\left|J_{2}\right|}\right) .
$$

Motivated by this statement, the structure factor was calculated for all wave vectors $\mathbf{q}=\left(q_{x}, q_{x}\right)$. In figure 6.9 a color coded plot of all discrete normalized structure factors $S\left(q_{x}, q_{x}\right)$ is shown for $L=20$ and $L=80$ for two cases $J_{1}=-0.5\left|J_{2}\right|$ and $J_{1}=+0.8\left|J_{2}\right|$. In the upper part for the small lattice $S(\mathbf{q})$ shows only a finite signal for two (left) and three (right) neighboring wave vectors, respectively. A saturated signal $S=1$ appears 


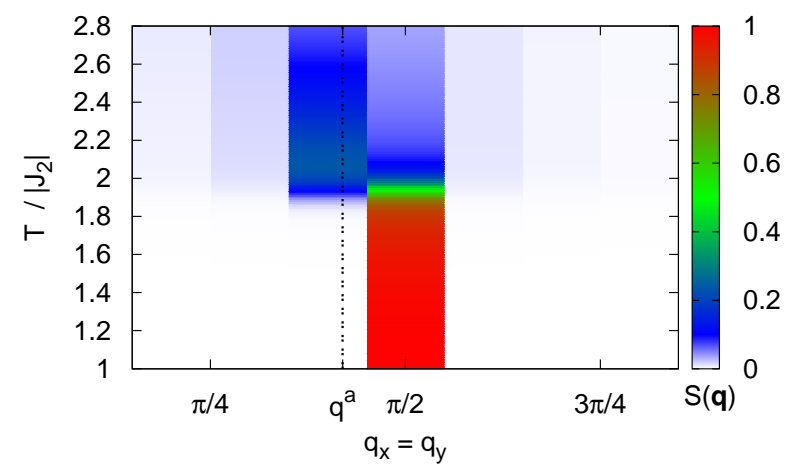

(a) $L=20, J_{1}=-0.5\left|J_{2}\right|$

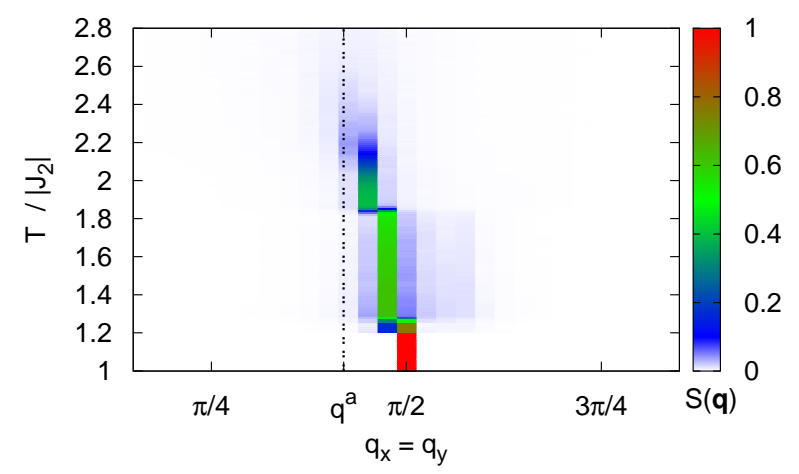

(c) $L=80, J_{1}=-0.5\left|J_{2}\right|$

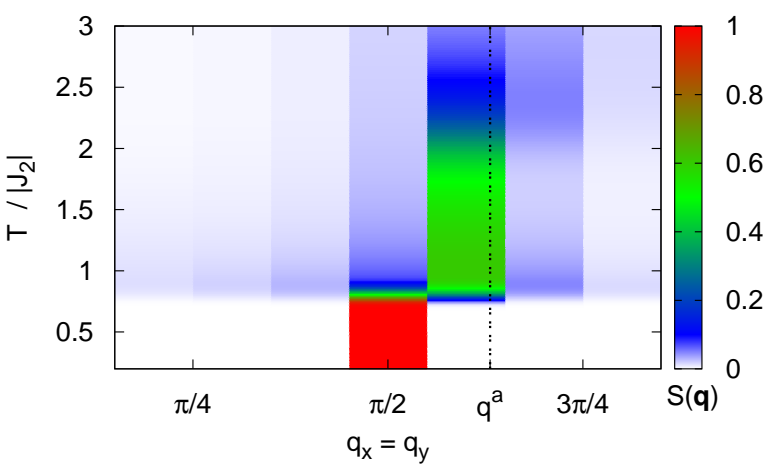

(b) $L=20, J_{1}=0.8\left|J_{2}\right|$

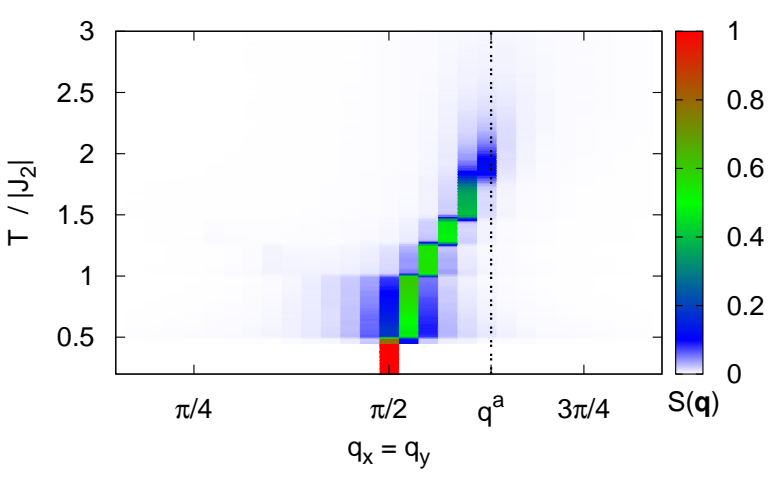

(d) $L=80, J_{1}=0.8\left|J_{2}\right|$

figure 6.9: Structure factors in color code for all $\mathbf{q}=\left(q_{x}, q_{x}\right)$ and different temperatures. The ground state is clearly given at $(\pi / 2, \pi / 2)$. The limit for the wave vectors is given by the value $q_{x}^{a}$ (confer equation (6.4) and [CG05]), however, for all values between $q_{x}^{a}$ and $\pi / 2$ a finite signal is observed as well.

for the ground state at $(\pi / 2, \pi / 2)$ only. For a larger lattice $(L=80$, figure 6.9 bottom) a whole cascade of signals appears in the structure factor; this is in agreement with the signatures in the correlation functions presented above. Furthermore, it is noteworthy that for a ferromagnetic coupling $J_{1}<0$ (figure 6.9 left) the wave vectors of the incommensurate ordering tend towards lower values, i.e., lie between the vectors indicating commensurate ferromagnetic and SFAF order. For an antiferromagnetic coupling (right) the opposite behavior is observed, $\pi / 2<q_{m}<\pi$. Thus, the unconventional order of the intermediate states stems from the competition of different ground states (SFAF order and Néel/ferromagnetic order) that are favored by the nearest and next-nearest neighbor coupling respectively. In addition two different magnitudes for the nearest-neighbor coupling were investigated: on the left-hand side a smaller absolute value of $\left|J_{1}\right|=0.5\left|J_{2}\right|$ yields a wave vector $\mathbf{q}^{a}$ which is closer to $(\pi / 2, \pi / 2)$ than $\mathbf{q}^{a}\left(\left|J_{1}\right|=0.8\left|J_{2}\right|\right)$ (right) and, thus, a smaller number of intermediate states is allowed for the large systems (figure 6.9 bottom). However, it is observed that the incommensurate order is not described by a single wave vector but rather a series of vectors $\mathbf{q}^{\text {inc. }}$ which satisfy two properties:

$$
\mathbf{q}^{\text {inc. }}=\left(q_{x}^{\text {inc. }}, q_{x}^{\text {inc. }}\right) \quad \text { and } \quad \begin{cases}q_{x}^{a} \leq q_{x}^{\text {inc. }}<\pi / 2 & \text { for } J_{1}<0 \\ \pi / 2<q_{x}^{\text {inc. }} \leq q_{x}^{a} & \text { for } J_{1}>0\end{cases}
$$

Thus, the obtained features could represent a devil's staircase [Bak82] which manifests itself in a sequence of signals for neighboring wave vectors for decreasing temperatures. 


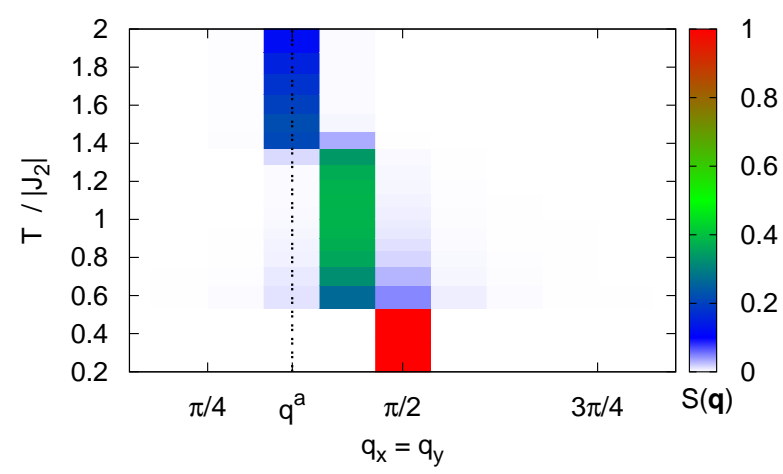

figure 6.10: Structure factor for $q_{x}=$ $q_{y}$ and a particular set of lattice size $L=$ 28 and nearest-neighbor interactions $J_{1}=$ $-0.867768\left|J_{2}\right|$ such that $q_{x}^{a}$ equals a lattice vector (confer (6.6)). The same behavior as for different $q_{x}^{a}$ is observed.

The condition (6.4) for $q_{x}^{a}$ can hold only in a finite lattice exactly for particular sets of values $J_{1}$ and $L$. To ensure that the above described behavior which includes multiple wave vectors is also valid for these special sets one additional plot is given in figure 6.10. The parameters are chosen such that $q_{x}^{a}$ equals a lattice vector of the finite lattice:

$$
L=28, \quad q_{x}^{\mathrm{SFAF}}=\frac{7}{14} \pi \text { and } q_{x}^{a}=\frac{5}{14} \pi \stackrel{(6.4)}{\rightarrow} J_{1}=-0.867768\left|J_{2}\right|
$$

The behavior is the same as for the non-fitting parameter sets, i.e., for the middle wave vector $q_{x}^{\text {inc. }}=\frac{6}{7} \pi$ a signal also appears at lower temperatures. However, the limiting property of $q_{x}^{a}$ (in this case as a lower bound for the wave vectors) is very prominent here.

$$
\begin{array}{l|l}
\text { summary } & 6.4
\end{array}
$$

An anisotropic version of the frustrated $J_{1^{-}} J_{2}$ Ising model was investigated using MonteCarlo simulations. In particular the phase transition into an antiferromagnetic state consisting of two sublattices in collinear order was analyzed. As predicted by Chitov and Gros [CG05] via mean-field theory, an incommensurate ordered phase appears for intermediate temperatures before the ground state is reached. The nature of this state was analyzed using correlation functions and the corresponding structure factors. In contrast to earlier statements the incommensurate order is not described by a single wave vector but rather a set of neighboring wave vectors resembling a devil's staircase on the finite lattice system.

The nature of the various phase transitions between the different incommensurate and commensurate states and the ground state was evaluated by means of energy histograms and Binder cumulants. On the one hand the energy histograms show very clear double peaks for all transitions; on the other hand all transitions are strongly dependent on the simulated system size, i.e., the transition temperatures are shifted to lower values for larger lattices and, hence, the Binder cumulants do not yield any crossing points. This particular behavior is more prominent for crossover phenomena rather than genuine phase transitions. However, this may be caused by the incommensurate order since the relevant wave vector is strongly temperature dependent and in the thermodynamic limit an interval of continuous momenta describes the incommensurate state. 


\section{disorder by quantum fluctuations}





\section{quantum disordered ground state for hard-core bosons on the frustrated square lattice}

The main content of this chapter was published under the same title in Physical Review B [KHFP11a]. Some additional notes were later published in the proceedings for the conference on strongly correlated electron systems (SCES 2011) [KHFP11b] with the same coauthors: Sebastian Fuchs who implemented some crucial improvements to the quantum Monte-Carlo code that was used for the simulations, Andreas Honecker as scientific advisor who performed the third- and fourth-order series expansion and Thomas Pruschke as additional scientific advisor. For the exact diagonalization an implementation of Jörg Schulenburg was employed [Sch].

It may be that some parts of the following chapter resemble the manuscript of reference [KHFP11a] which has been written mainly by me and was revised by Andreas Honecker and the two other coauthors.

\section{abstract}

In this chapter the ground-state phase diagram for a model of hard-core bosons on a frustrated square lattice is investigated. The model is equivalent to an anisotropic version of the frustrated $J_{1^{-}} J_{2}$ spin-1/2 Heisenberg model. Starting at a classical limit of zero quantum fluctuations two different methods - series expansions and quantum Monte-Carlo - are applied to analyze the stability of classical ordered states against the influence of small fluctuations. In the opposite limit - large kinetic energy of the bosons - a superfluid phase is identified. However, in the vicinity of the critical point of this frustrated model a finite region in the phase diagram is found where no conventional order arises. The analysis of higher order correlation functions using quantum Monte-Carlo and the evaluation of the low-energy spectrum gained from exact diagonalization indicate a quantum-disordered ground state.

The Hamiltonian of the model was introduced in section 2.3 in the boson and spinoperator description. The content of this chapter mainly refers to the spin- $1 / 2$ Heisenberg model with parameters $t_{i}$ and $V_{i}$ :

$$
\begin{aligned}
H_{\mathrm{HM}}= & t_{1} \sum_{\mathrm{NN}}\left(S_{i}^{+} S_{j}^{-}+\text {H.c. }\right)+V_{1} \sum_{\mathrm{NN}} S_{i}^{z} S_{j}^{z} \\
& +t_{2} \sum_{\mathrm{NNN}}\left(S_{i}^{+} S_{j}^{-}+\text {H.c. }\right)+V_{2} \sum_{\mathrm{NNN}} S_{i}^{z} S_{j}^{z} .
\end{aligned}
$$

The model resides on a $N=L \times L$ square lattice with periodic boundary conditions. The $V_{i}>0$ are chosen antiferromagnetic and $V_{2}$ introduces frustration into the model. The fluctuation parameters $t_{i}<0$ are negative and represent ferromagnetic and, hence, non-frustrating spin exchange. 


\section{methods}

In the classical limit, i.e., for $t_{i}=0$, the Hamiltonian represents the antiferromagnetic $J_{1^{-}} J_{2}$ Ising model $\left(J_{i}=V_{i}\right)$ which is highly frustrated in the region $J_{2} \approx J_{1} / 2$ and was discussed in the chapter 5 . The stability of the classical ground states - Néel order for $J_{2}<J_{1} / 2$ and collinear order for $J_{2}>J_{1} / 2$ - is tested by means of quantum MonteCarlo simulations for fluctuations $t_{i}<0$. At the frustrated point $V_{2}=V_{1} / 2$ perturbative derivations are performed to calculate the direct transition between the two perturbed classical states. For large $\left|t_{i}\right|$ a ferromagnetic long-range correlation in the $x y$ plane is expected. This corresponds to phase for the hard-core bosons with a finite superfluid density which is calculated within the Monte-Carlo simulations.

For the most interesting region (intermediate $\left|t_{i}\right|$ and $V_{2} \approx V_{1} / 2$ ) the emergence of new quantum states will be investigated by means of quantum Monte-Carlo and exact diagonalization.

\section{Ising limit $\mid 7.1$}

The purely classical ground states of the Ising limit are no eigenstates of the quantum Hamiltonian given in (7.1) as the spin-exchange terms are off-diagonal in the $S^{z}$-product basis. However, for small fluctuations the quantum mechanical ground state may show a finite but reduced signal in the order parameter of the original antiferromagnetic states. ${ }^{1}$ The temperature-dependent behavior of the order parameter and accordingly the Binder cumulant can be calculated with quantum Monte-Carlo simulations in the same manner as in the classical case.

\section{quantum Monte-Carlo}

For the present work an implementation of the stochastic series expansion [Han62, San92, SK91, see also section 3.2.2] with a directed-loop update [SS02] from the ALPS project $\left[\mathrm{AAC}^{+} 07, \mathrm{ADG}^{+} 05, \mathrm{AWT} 05\right]$ was used for all quantum Monte-Carlo simulations.

However, the frustration in the model produces a critical slowing down in the MonteCarlo simulation and the large degeneracy in the vicinity of the critical point $V_{2}=V_{1} / 2$ causes severe thermalization problems in the standard implementation. To overcome these problems an exchange Monte-Carlo update [HN96, KTHT06, Mel07, see also section 3.2.2] in temperature space was added by Sebastian Fuchs to the ALPS directed-loop application. To guarantee a good thermalization within the exchange Monte-Carlo step it is important to adjust the temperature steps and number of sweeps between the exchanges of configurations (swaps) of neighboring simulations.

In addition to the exchange Monte-Carlo, an annealing procedure was performed for each copy independently during the thermalization process. This kind of algorithm helps to prethermalize the simulations at lower temperatures to ensure a better swap rate for the exchange Monte-Carlo algorithm.

It is noteworthy that the accessible system sizes for the quantum model are much smaller than for the classical case due to the higher complexity of the quantum MonteCarlo simulation. However, the finite-size effects are reduced by the influence of the

\footnotetext{
${ }^{1}$ In the following the antiferromagnetic states will be referred to as Néel and collinear state as in the classical case, although they are not eigenstates.
} 


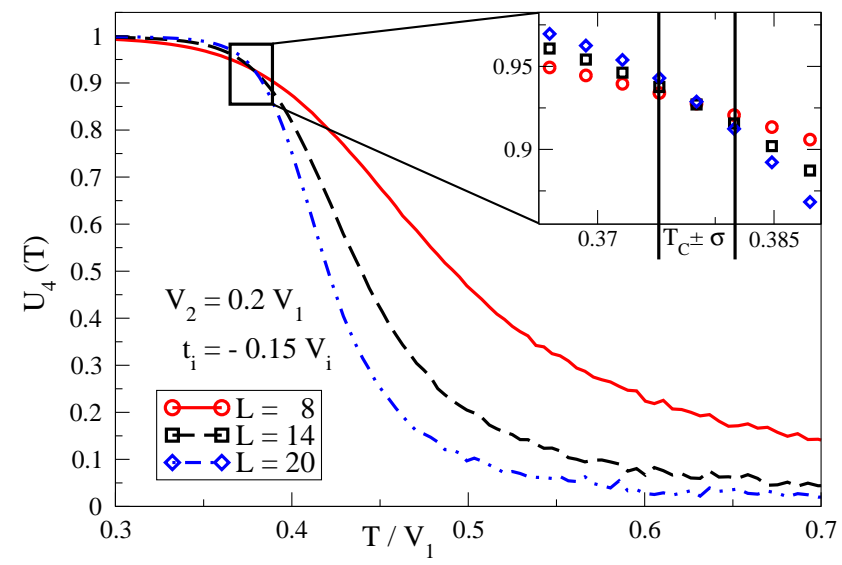

figure 7.1: (Slightly different version published in [KHFP11a].) Temperature dependence of the fourth-order cumulants for the Néel order parameter for different lattice sizes at $V_{2}=0.2 V_{1}$ and $t_{i}=$ $-0.15 V_{i}$. In the inset the estimate of the error for the transition temperature $T_{C}=0.378(3) V_{1}$ is given .

fluctuations and already for small systems a reliable estimation of transition temperatures is possible.

The static structure factors introduced in section 2.1 can easily be evaluated in the $S^{z}$ configuration space of the quantum Monte-Carlo simulation. For example, the Binder cumulants of the Néel-order parameter for different lattice sizes $L \leq 20$ are shown in figure 7.1 ; as in the classical case the intersection point yields the critical temperature $T_{C}\left(V_{2} / V_{1}\right)$.

A comparison of the critical temperatures at fixed frustration $V_{2} / V_{1}$ for different values of $t_{i}$ shows that the influence of quantum fluctuations reduces the temperature at which the phase transition from the disordered paramagnetic state to the ground state with antiferromagnetic long-range correlations takes place. Thus, classical order is suppressed by quantum fluctuations. In figure 7.2 the finite-temperature phase diagram is presented for different $t_{i}$. It is already notable that close to the highly frustrated point the critical temperatures decrease more drastically and are completely suppressed for large $\left|t_{i}\right|>$ $0.10 V_{i}$.

Indeed, the Néel and collinear state are no longer the ground states for increasing quantum fluctuations and close to the critical point $V_{2}=V_{1} / 2$ a detailed analysis of the direct transition between both states is necessary. Therefore, the energies of both antiferromagnetic states are compared by means of a perturbational ansatz: a series expansion of the quantum fluctuations perturbing the classical Ising model as sketched in section 4.1 .

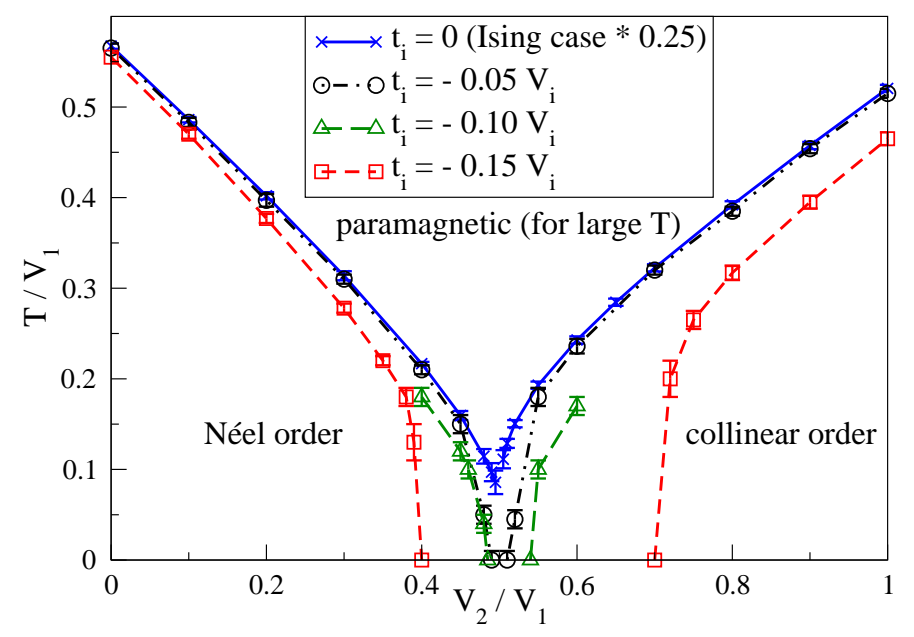

figure 7.2: $\quad$ (Published in [KHFP11a].) Phase diagrams at finite temperatures for different values of $t_{i}$. For increasing $\left|t_{i}\right|$ the transition temperatures are shifted to lower values and conventional order is suppressed. To guide the eye, the full (blue) line shows the known phase boundary for the classical appropriately scaled Ising model [KHFP08, see also chapter 5]. For $t_{i}=-0.1 V_{i}$ (broadly dashed (green) line) only transition temperatures close to the critical point were calculated. 


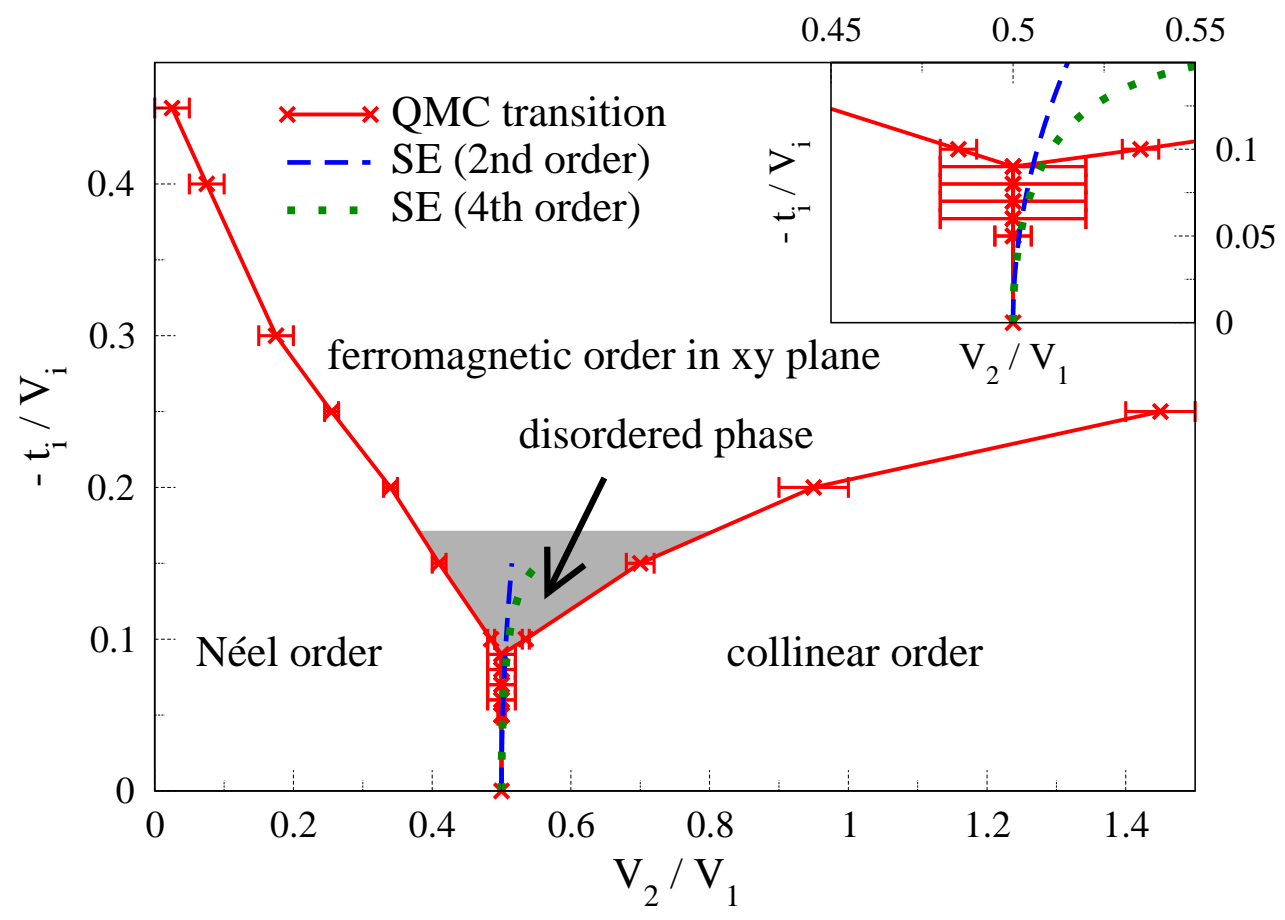

figure 7.3: (Published in [KHFP11a] without fourth-order SE and inset.) Ground-state phase diagram calculated with quantum Monte-Carlo simulations. On the horizontal axis the degree of frustration $V_{2} / V_{1}$ is plotted, and on the vertical line the magnitude of the quantum fluctuations $-t_{i} / V_{i}$ is plotted. The dashed blue line and dotted green line indicate the direct phase boundary between Néel and collinear order derived by means of series expansion (SE, see section 7.1). The gray area represents the approximate region where no finite signal in the conventional order parameters arises. In the inset a small section around $V_{2}=V_{1} / 2$ is shown in a larger scale to illustrate the series expansion results more clearly.

\section{perturbation theory}

To estimate the influence of small quantum fluctuations on the classical ground states, second-order perturbation terms are calculated in the degenerate ground state manifold at the critical point $V_{2}=V_{1} / 2$ [BSFB07, SO83]. For $V_{2}=V_{1} / 2$ every state with $S_{\text {total }}^{z}=0$ per plaquette (square of 4 lattice sites) has the same classical energy. Thus, for the whole lattice, which consists of overlapping plaquettes, this local degeneracy yields a global degeneracy of the order of the lattice length $L$, as explained in reference [KHFP08] for the classical limit. The degenerate perturbation theory distinguishes between diagonal perturbations which leave the system in exactly the same state and off-diagonal perturbations which transfer the system into another state of the degenerate manifold. In the case of the frustrated square lattice different ground states are connected via flips of antiferromagnetic spins in a whole line or row of the lattice. Thus, the order of offdiagonal perturbations scales with the length of the lattice $L$. Off-diagonal perturbations are therefore negligible in the thermodynamic limit with $L \rightarrow \infty$. However, non-zero diagonal perturbations appear already in the second order and are different for the two classical starting points - the Néel and collinear configurations. In the Néel state only nearest-neighbor hopping $t_{1}$ is possible on $2 L^{2}$ bonds of the lattice and thus yields an energy gain of $\Delta E_{1}=-2 L^{2} \frac{t_{1}^{2}}{V_{1}}$. In the collinear state nearest-neighbor hopping on $L^{2}$ bonds and next-nearest-neighbor hopping on $2 L^{2}$ bonds is possible which gives an energy gain $\Delta E_{2}=-2 L^{2} \frac{t_{2}^{2}}{3 V_{2}}-L^{2} \frac{t_{1}^{2}}{V_{1}}$. Thus, small fluctuations decrease the energy of the 
ground states in the vicinity of the critical point. Calculating the transition line between the Néel and collinear state - taking into account only second-order corrections to the classical energies and using $t_{2} \approx t_{1} / 2$ and $V_{2} \approx V_{1} / 2$ - yields the relation

$$
\frac{\left|t_{i}\right|}{V_{i}}=\sqrt{\frac{3}{2} \frac{V_{2}}{V_{1}}-\frac{3}{4}}
$$

which is shown in the final ground state phase diagram (figure 7.3) as the dashed blue line for small $\left|t_{i}\right| / V_{i}$. Because equation (7.2) does not depend on the sign of $t_{i}$ it also holds for antiferromagnetic $x y$ interactions and can be compared to the result of a series expansion by Oitmaa et al. [OW96] where for positive fluctuations $t_{i}>0$ the direct transition between the classical antiferromagnetic states survives in this completely frustrated model as well for small fluctuations.

Higher-order perturbations (up to fourth-order) were calculated by Andreas Honecker also away from the critical point, i.e., a series expansion for all values of $V_{2} / V_{1}$. By equating the energy functions - given by rational functions with higher order dependencies in $t=t_{i} / V_{i}$ and $V=V_{2} / V_{1}$ and expanded around $V_{2}=V_{1} / 2$ - at the critical point a more accurate estimation of the direct transition is obtained

$$
V(t)=\frac{1}{2} \frac{2730859 t^{4}-388800 t^{3}+81000 t^{2}-8100}{2802739 t^{4}-40400 t^{3}+91800 t^{2}-8100}
$$

which is also shown in figure 7.3 as the green dotted line. The difference between both lines is clearly visible in the inset of figure $7.3 .^{2}$

The results for the energies for different values of $t$ and $V$ are compared below to the results of Monte-Carlo simulations.

$$
\begin{array}{l|l}
\text { ferromagnetic limit } & 7.2
\end{array}
$$

The parameters $t_{i}$ scale the magnitude of the spin fluctuations that correspond in the original notation of the Hamiltonian [confer, e.g., (2.24)] to the magnetic exchange in the $S^{x y}$ components. Thus, for negative $t_{i}$ a ferromagnetic configuration in the $x y$ plane reduces the overall energy. In section 2.3.2 a variational product ansatz for such a ferromagnetic order was given and yields an energy

$$
E_{\text {ferro }} / N=\left(t_{1}+t_{2}\right) .
$$

At the point $V_{1}=V_{2}=t_{2}=0$ and $t_{1}=-0.5$ the energy $e_{\text {ferro }}=-0.5$ can be compared to the pure $x y$ model, i.e., an anisotropic Heisenberg model without $S^{z}$ interactions and only nearest-neighbor interactions, from reference [Jia11, SH99]: energy $e_{x y}=-0.548824(2)$. This shows that already for the non-frustrated case the approximation of the product ansatz does not fit very well. The order parameter for this phase requires a more subtle definition since the quantum Monte-Carlo operates in the $S^{z}$ basis. The observable was presented in section 3.2.3 and is called - based on its bosonic origin - superfluid density $\rho_{S}$ (or spin stiffness). In figure 7.4 the development of $\rho_{S}$ is shown for (apart from the sign) isotropic choice $t_{i}=-V_{i} / 2$ and $V_{2}=V_{1} / 2$. The convergence of the order parameter for different lattice sizes $(L=8,14)$ proves the long-range ferromagnetic order. The absolute

\footnotetext{
${ }^{2}$ An expansion of equation (7.3) up to second order in $t$ around $t=0$ yields the relation (7.2) again.
} 


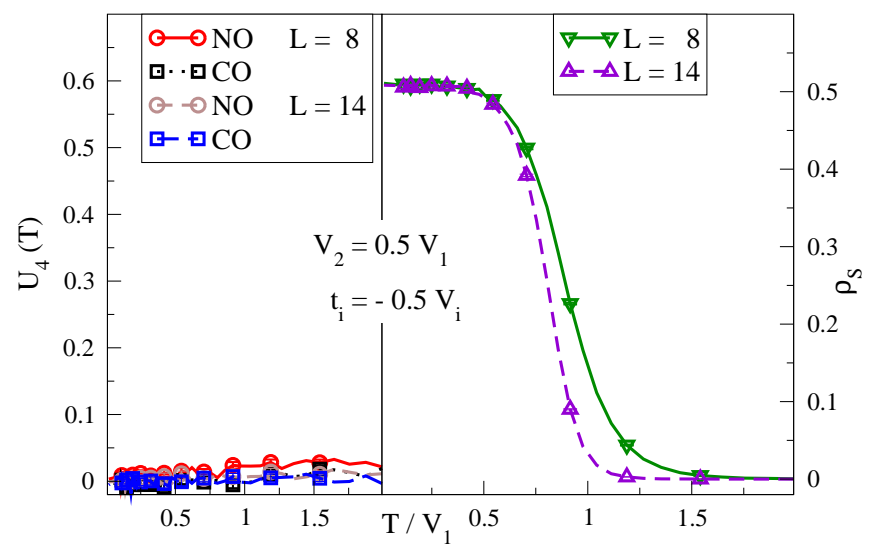

figure 7.4: (Published in [KHFP11a].) Comparison of the superfluid density (spin stiffness) $\rho_{S}$ (right) and cumulants for antiferromagnetic order parameters (left) - Néel order (NO) and collinear order $(\mathrm{CO})-$ for $t_{i}=-V_{i} / 2$ and $V_{2}=$ $V_{1} / 2$. The clearly converged signature in the superfluid order parameter indicates a finite-temperature phase transition.

value for $\rho_{S}=0.508(2)$ can be compared to the result for the $x y$ model $\rho_{x y}=0.26974(5)$. Hence, the additional ferromagnetic coupling on the next-nearest neighbor bonds enhances the signal in the order parameter. In conclusion, evidence for long-range magnetic order in the two limits $\left|t_{i}\right| \nearrow V_{i} / 2$ (ferromagnetic in-plane order) and $\left|t_{i}\right| \searrow 0$ (antiferromagnetic Néel and collinear order) is found.

The calculations for all three order parameters at a medium value $t_{i}=-0.25 V_{i}$ and varying frustration is shown in figure 7.5. Also included are the energies obtained by quantum Monte-Carlo and series expansion up to fourth-order. The agreement for the energies from series expansion and quantum Monte-Carlo is very good in the region where antiferromagnetic order is present. For the remaining values of $V_{2} / V_{1}$ the quantum MonteCarlo yields a lower energy than the series expansion.

However, for even smaller values of $\left|t_{i}\right|$ and intermediate range of frustration the convergence of the ferromagnetic order parameter breaks down and only for small systems a residual signal is found for intermediate temperatures. Thus, the ferromagnetic correlations are not stable in a finite-size analysis as demonstrated in figure 7.6, where the energy converges to its ground state value.

A finite-size analysis for all magnetic order parameters is necessary in some finite parameter region to exclude the conventional order of the introduced limits. Therefore, in figure 7.7 order parameters and energies for two different parameter scans are presented: (a) at fixed ratio $t_{i}=-0.1 V_{i}$ a range of $V_{2} \sim V_{1} / 2$, i.e., close to the critical point, and (b) at the critical point $V_{2}=V_{1} / 2$ a set of increasing values of $0 \leq\left|t_{i}\right| / V_{i} \leq 0.5$.

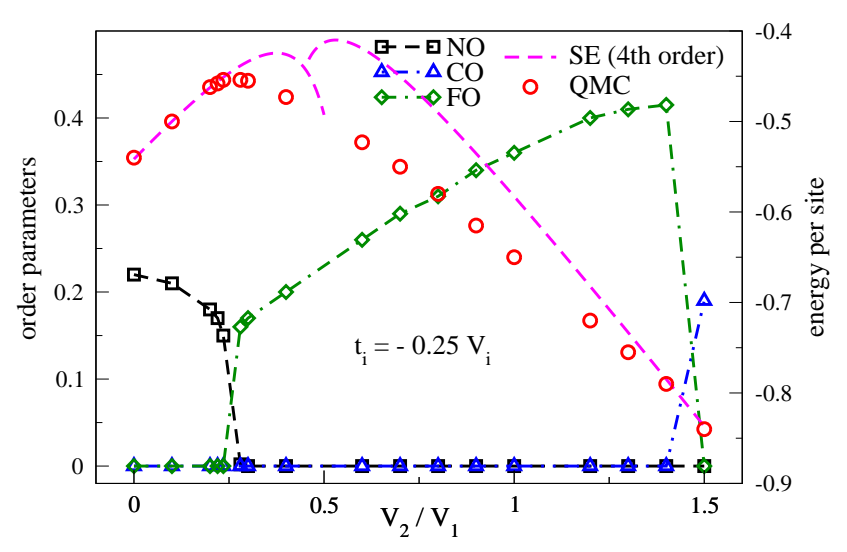

figure 7.5: (Published in [KHFP11a] without series expansion (SE).) Groundstate values of the order parameters for Néel order (NO), collinear order (CO), in-plane ferromagnetic order $(\mathrm{FO})$ and the energy density (from quantum MonteCarlo (QMC) and SE for both Néel and collinear configuration) at $t_{i}=-0.25 V_{i}$ and varying frustration $V_{2} / V_{1}$. The agreement for the energies is very good in the region where antiferromagnetic $S^{z}$ order is stabilized. (Symbols are larger than error bars and values converge for $20 \geq L \geq 16$ and $\left.T \leq 0.02 V_{1}\right)$. 
From figure $7.7 \mathrm{a}$ it is clear that the system for small values $t_{i}$ neither orders in the antiferromagnetic Ising states nor shows long-range ferromagnetic correlations in the $x y$ plane. The comparison of the energies from quantum Monte-Carlo and series expansion which is based on the Ising ground states enforces the breakdown of antiferromagnetic order for the given values of $V_{2} / V_{1}$. An estimation of the finite region in the ground-state phase diagram where no conventional order is stabilized can be extracted from figure 7.7b. At $V_{2}=V_{1} / 2$ the phase boundary between the antiferromagnetic states splits at $t_{i}=-0.08(1) V_{i}$. However, the above mentioned size dependence of the superfluid order parameter permits only a rough evaluation of the upper critical value of $t_{i}=-0.175(25) V_{i}$. In addition, the energy of the simple variational ansatz (7.4) is compared to the groundstate energies obtained by quantum Monte-Carlo. The agreement is rather bad which is explained by the complete neglect of quantum fluctuations in the ansatz. The energy $e_{S}=-0.8808(2) V_{1}$ of the ferromagnetic state at $V_{2}=V_{1} / 2$ and $t_{i}=-0.5 V_{i}$ can again be compared to the value for the $x y$ model with only nearest-neighbor interactions from [SH99]: $e_{x y}=0.548824(2) J$. The energy is significantly decreased by the influence of the additional coupling on the next-nearest neighbor bonds.

Combining all results, a ground-state phase diagram can be drawn which is shown in figure 7.3. The shape of the phase diagram is very similar to the one obtained by Oitmaa et al. in reference [OW96] for positive values $t_{i} / V_{i}$. They argued that between the two antiferromagnetic states a spin-liquid phase arises above some value of $t_{i}$.

The phase transitions between the different ground states are not analyzed in detail but from the classical case it is known that the direct transition between both antiferromagnetic states is of first order. The behavior of all three order parameters, shown in figure 7.5, at the transitions to the ferromagnetic phase also hints towards first-order transitions, as the onset of the different observables is rather abrupt. For the remaining phase boundaries with the unidentified phase an analysis of figure 7.7 helps. According to the same argument the transition from the antiferromagnetic phases is discontinuous. However, the transition into the ferromagnetic phase seems to be rather smooth and is probably continuous. Due to the strong finite-size dependence of the system in this region a reliable investigation of the phase transition, e.g., the calculation of critical exponents, is not possible at this point. However, a more detailed analysis of the unidentified phase is presented in the following section.

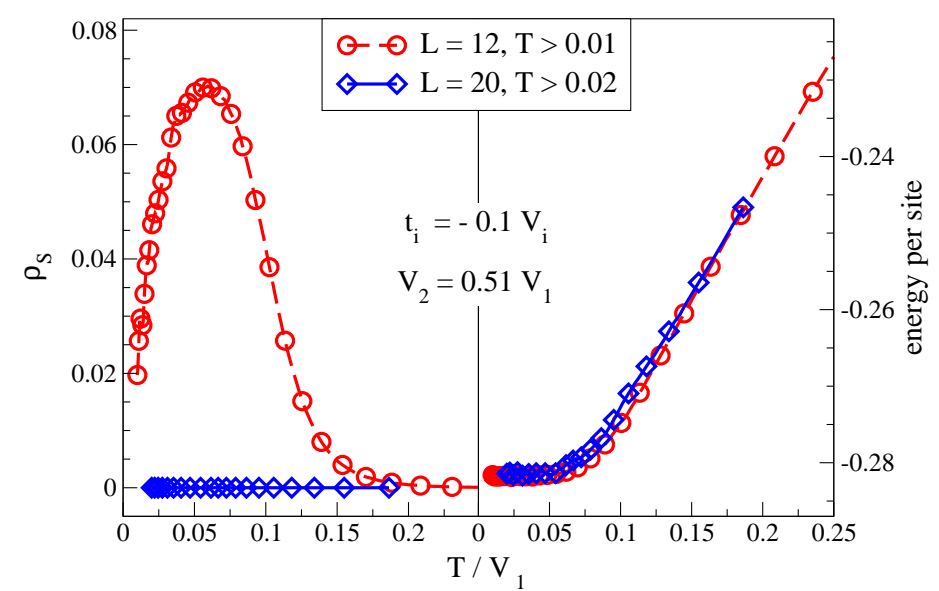

figure 7.6: (Published in [KHFP11b].) The superfluid density $\rho_{S}$ shows a small signal for an intermediate temperature only in the $12 \times 12$ lattice at $V_{2}=0.51 V_{1}$ and $t_{i}=0.1 V_{i}$. For a larger lattice no finite signal is found (left) where the energies are saturated to their ground state value for $T<0.05 V_{1}$ (right). 


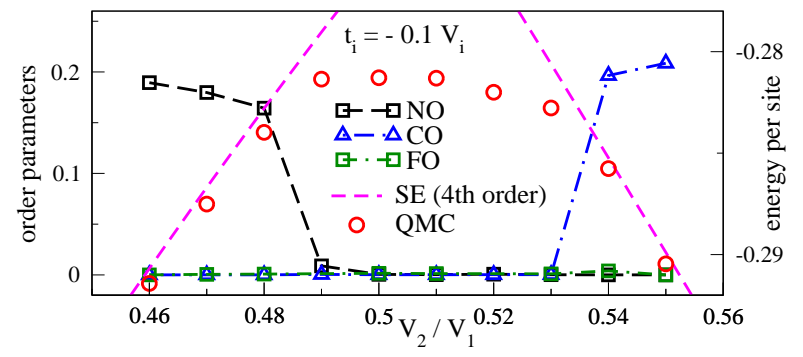

(a) $t_{i}=-0.1 V_{i}$ and varying frustration

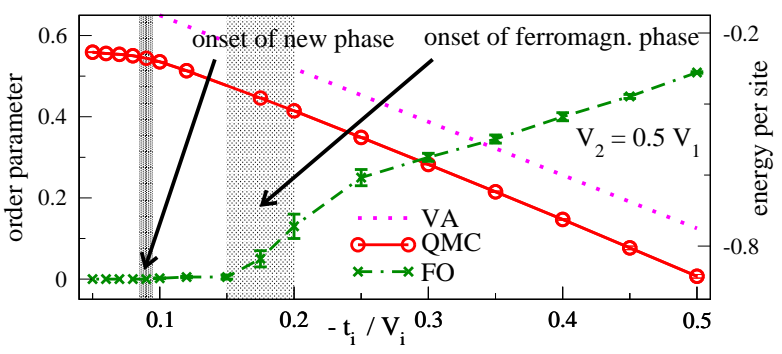

(b) $V_{2}=V_{1} / 2$ and increasing fluctuations

figure 7.7: (Published in [KHFP11a] without SE and variational ansatz (VA), respectively.) Ground-state values of the order parameters for Néel order (NO), collinear order (CO), in-plane ferromagnetic order (FO) and the energy density for two different parameter scans. (a) A finite region without conventional order is identified for the intermediate value of $t_{i}=-0.1 V_{i}$. (b) The lower bound $t_{i}=-0.08(1) V_{i}$ is given by the opening of the two antiferromagnetic phases which can be calculated accurately. The upper bound $t_{i}=-0.175(25) V_{i}$, however, is given by the onset of the superfluid order parameter (FO) which exhibits large errors obtained by finite-size scaling. Furthermore, the energy of the variational ansatz (VA) given in equation (7.4) is shown and well above the Monte-Carlo values. (Symbols are larger than error bars (if not given) and values are converged for $20 \geq L \geq 16$ and $T \leq 0.02 V_{1}$.)

\section{\begin{tabular}{l|l} 
intermediate regime & 7.3
\end{tabular}}

The lack of conventional order in a finite region of the ground state phase diagram motivated further simulations and calculations of new order parameters. Since from classical frustrated models it is known that incommensurate ordering may also occur for certain parameters (see previous chapter), a calculation of the complete structure factor for a finite $20 \times 20$ lattice was performed. The ground-state result for $t_{i}=-0.1 V_{i}$ and $V_{2}=0.51 . V_{1}$ is shown in figure 7.8 for all momenta $\mathbf{q}=\left(q_{x}, q_{y}\right)$ with $0 \leq q_{i} \leq \pi$. Apart from a small enhancement of the signal for larger $q_{i}$ which originates from the finite size of the lattice and the non-zero temperature $T=0.02 V_{1}$ the flat structure factor rules out any magnetic order - of commensurate or incommensurate type - in $S^{z}$ direction.

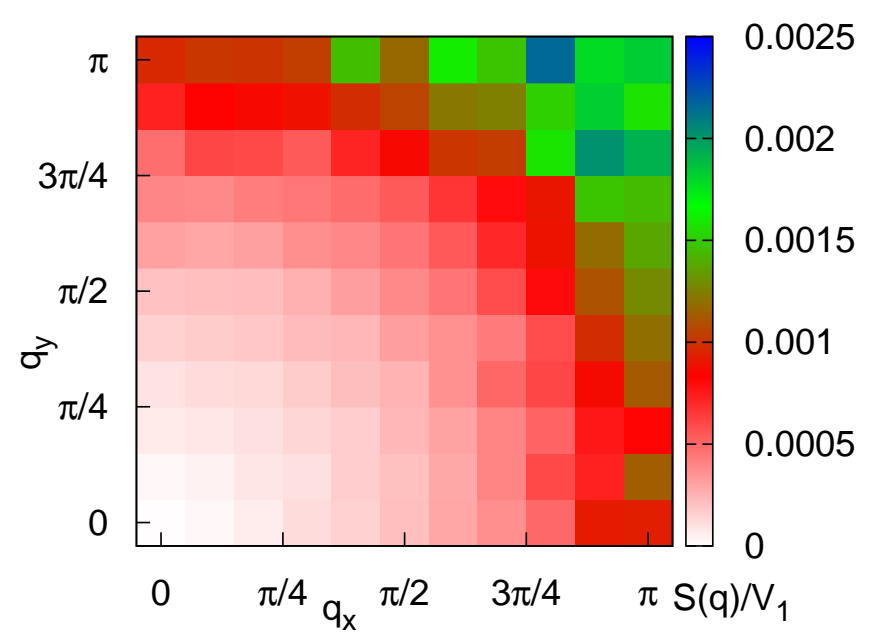

figure 7.8: For $t_{i}=-0.1 V_{i}$ and $V_{2}=0.51 V_{1}$ the complete structure factor of a $20 \times 20$ lattice is shown and only a vanishingly small signal at $\mathbf{q} \approx(\pi, \pi)$ is identified - probably due to finite-size effects and the finite temperature $\left(T=0.02 V_{1}\right)$. 


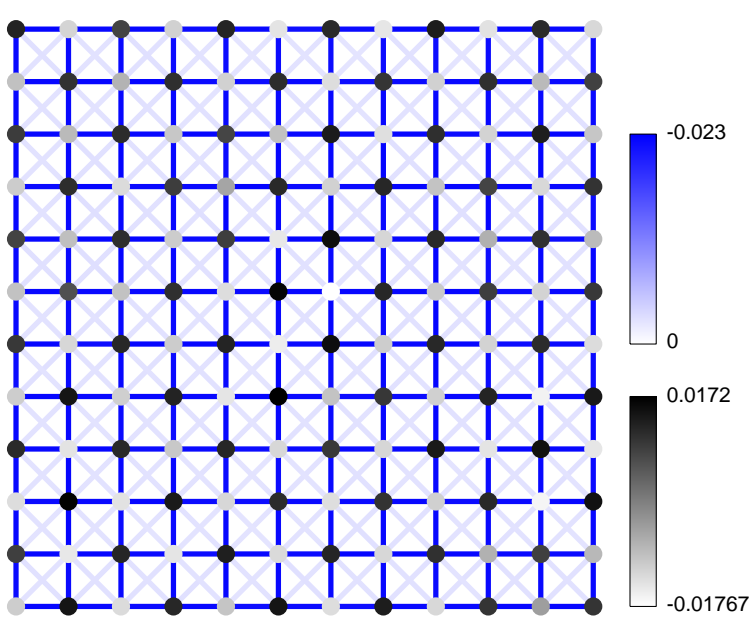

(a) $V_{2}=0.46 V_{1}$ (Néel region)

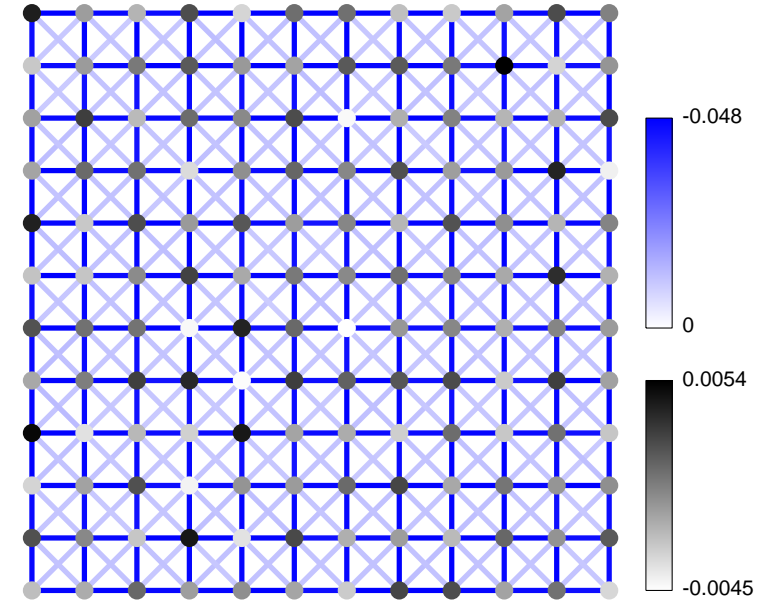

(b) $V_{2}=0.51 V_{1}$ (disordered region)

figure 7.9: Local measurements of magnetization and kinetic bond energy in gray (magnetization, small absolute values) and blue (bond energy) scale for a $12 \times 12$ lattice at $t_{i}=-0.1 V_{i}$ in the ground state $\left(T=0.01 V_{1}\right)$. (a) In the Néel phase a residual staggered magnetization and only small kinetic energies are visible. (b) The local magnetization shows very small disordered signals and the kinetic energy is enhanced. However, the ratio between nearest and next-nearest bond energies is smaller than the ratio $t_{2} / t_{1}=V_{2} / V_{1}=0.51$.

\section{quantum correlations}

As explained in the introduction of this thesis, a particular interest in frustrated quantumspin models is the possible emergence of quantum mechanically ordered and disordered phases. Possible configurations of such phases - like columnar or staggered dimer order - were introduced in section 2.3.2 and the derivation of an appropriate estimator of their order parameter in the quantum Monte-Carlo, i.e., an estimator of a four-spin correlation function

$$
\left\langle\mathbf{S}_{i} \cdot \mathbf{S}_{j} \mathbf{S}_{k} \cdot \mathbf{S}_{l}\right\rangle
$$

with $i-j$ and $k-l$ indexing sites of nearest-neighbor bonds, was given in section 3.2.3. For these phases both the potential energy from the $S^{z}$ interactions and the kinetic energy that stems from the fluctuation terms are important. For this reason the local average magnetization and kinetic energy of each site and bond respectively, in gray and blue scale for two different cases at $t_{i}=-0.1 V_{i}$ is shown in figure 7.9. The local magnetization yields nearly zero for all sites for both cases - Néel order (a) and disordered region (b) as expected due to the two-fold degeneracy of the Néel state and the vanishing structure factor in the disordered region. A residual Néel configuration can be seen in figure 7.9a on a very small scale. The local energies are equally distributed in both cases and no particular ordering appears. However, the average local kinetic energy is enhanced in the disordered phase by a factor of two by comparing the numbers on the upper scale, although the exchange parameters for nearest-neighbor fluctuations are the same for the two cases. It is noteworthy that a further analysis of the kinetic energies on nearest and next-nearest neighbor bonds yields different ratios $e_{\mathrm{NN}}^{\text {kin. }} / e_{\mathrm{NNN}}^{\text {kin. }}$ for the cases of a Néel ordered, ferromagnetic ordered and disordered system. In the Néel ordered phase the fraction equals approximately $20 \%$ of the actual ratio of the exchange parameters $t_{2} / t_{1}$, whereas in the ferromagnetic region a fraction of roughly $60 \%$ is obtained. For large 


\section{- Reference site and bond (top left corner)}

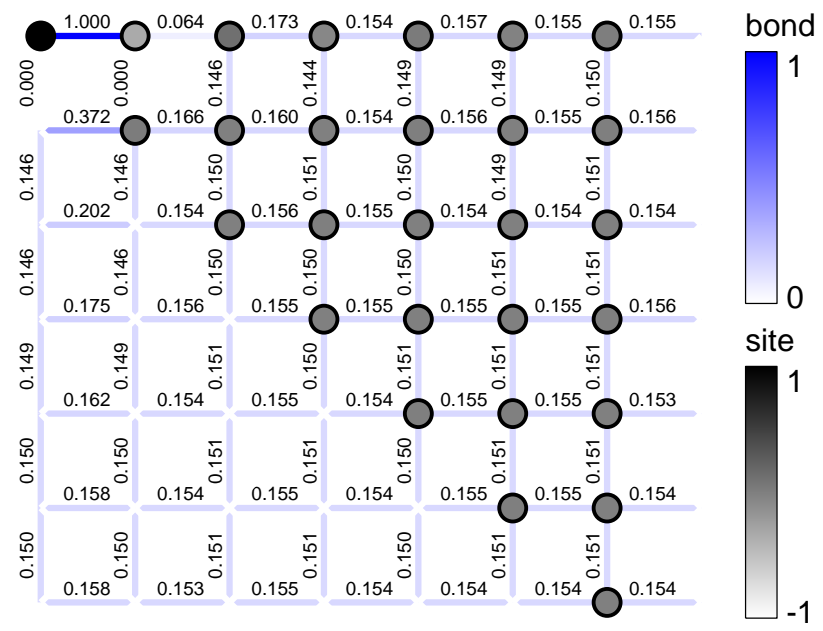

figure 7.10: (Published in [KHFP11a] in gray scale.) Correlations between spins and dimers sited on nearestneighbor bonds on a periodic $12 \times 12$ lattice; hence, up to six neighboring spins and bonds are shown. The top left site and its adjacent right bond yield the reference points for the measurement. All other sites and bonds of the plot represent the correlations of spins and dimers in the illustrated distance to the reference site/bond coded in gray and blue scales, respectively. For $V_{2}=0.51 V_{1}$ and $t_{i}=-0.1 V_{i}$, i.e., in the region without conventional order, the ground state $\left(T=0.01 V_{1}\right)$ exhibits no longrange order, indicated by the rapidly decaying correlations functions of spins and dimers.

lattices, i.e., $L=16,20$ with parameters inside the disordered region, a rate of $\sim 35 \%$ remains. Thus, in the disordered phase the kinetic energy on nearest-neighbor bonds is enhanced compared to the next-nearest neighbor bonds.

Motivated by this fact, the correlation of dimers residing on nearest-neighbor bonds were calculated as described in 3.2.3. The result is shown in figure 7.10 on a representative lattice for parameters in the disordered region of the phase diagram: all sites and bonds of the lattice represent in gray and blue scale, respectively, the strength of the correlations of spins and dimers in the given distance to the spin and dimer located on the top left site and bond. The autocorrelation of the top left dimer yields the normalization for the dimer correlations. The rapid decay of the $S^{z}$ spin correlations to zero confirms the absence of any magnetic order in the $z$ direction at $t_{i}=-0.1 V_{i}$ and $V_{2}=0.51 V_{2}$, as was seen before in the calculations for the structure factor (figure 7.8) and for the local magnetization (figure $7.9 \mathrm{~b}$ ). However, more interesting are the dimer correlations. The autocorrelation is larger than all other correlations to different dimers. Beyond that only a short-range correlations are present, i.e., for bonds in the direct vicinity of the reference bond (top left bond in figure 7.10) a reduction (perpendicular bonds and the fist parallel neighbor bond) and an enhancement (other parallel bonds) are detected. For larger distances (three bonds) all correlations on parallel and perpendicular bonds decay to a constant value and, hence, no long-range dimer order is present. This also agrees with the constant local kinetic energies on all bonds shown in figure $7.9 \mathrm{~b}$. The same applies for the results shown in figure 7.11 for $L=16$ at (a) $t_{i}=-0.12 V_{i}$ and $V_{2}=V_{1} / 2$ and (b) $t_{i}=-0.15 V_{i}$ and $V_{2}=V_{1} / 2$. Thus, all evidence points to a quantum disordered ground state in the whole region of the phase diagram without conventional magnetic order (gray area in figure 7.3 ). 


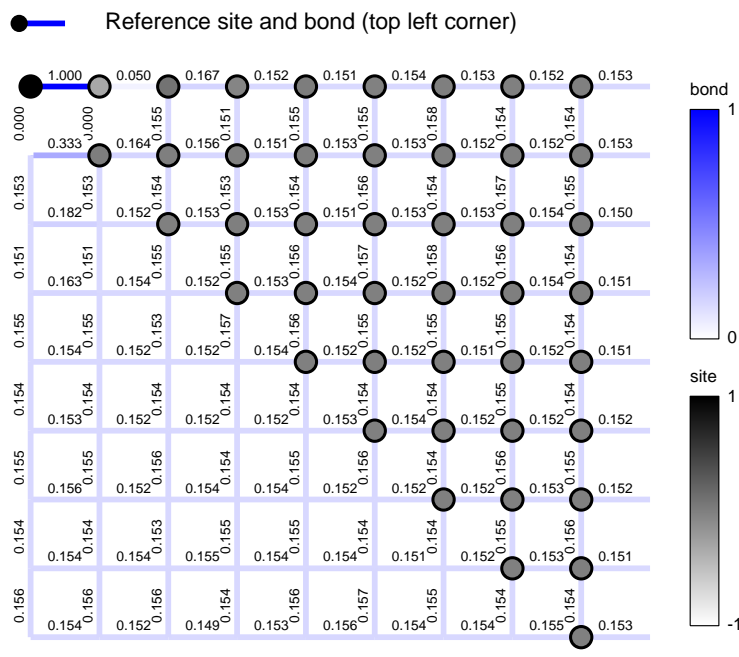

(a) $t_{i}=-0.12 V_{i}$

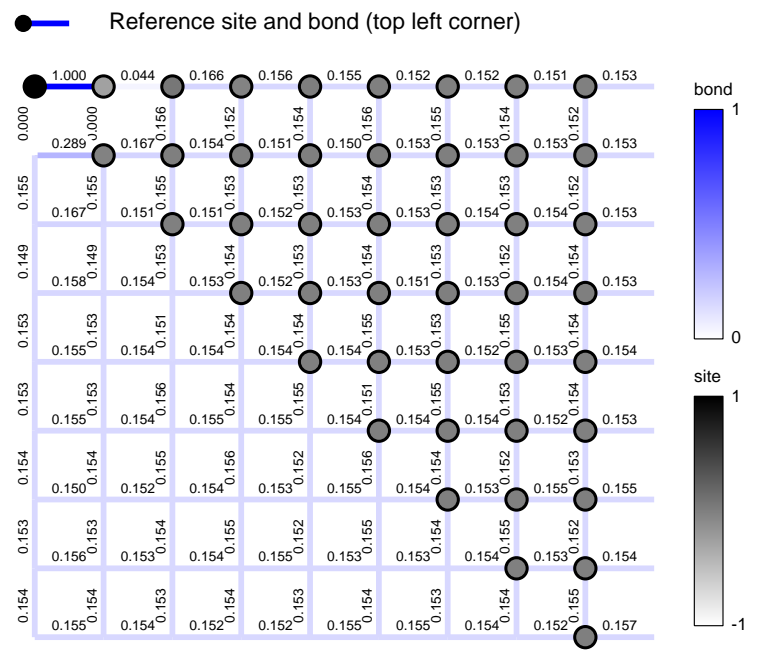

(b) $t_{i}=-0.15 V_{i}$

figure 7.11: Spin and dimer correlations for $16 \times 16$ lattices at $V_{2}=V_{1} / 2$ in the ground state $\left(T=0.01 V_{1}\right)$. No long-range order is present for dimers residing on nearest-neighbor bonds. ((a) was published in [KHFP11b].)

\section{low-energy spectrum ${ }^{3}$}

A topologically ordered state could be another possibility for the phase without any signatures for order. To check for this kind of non-local ordering, the calculation of the spectrum is necessary because a degeneracy of the ground state is expected for a topologically ordered state on a symmetric periodic lattice with an aspect ratio close to one [ML05, MLMS02]. The spectrum is not accessible via quantum Monte-Carlo simulations and therefore an exact diagonalization is performed for a $6 \times 6$ lattice with periodic boundary conditions - as on a torus - at $t_{i}=-0.1 V_{i}, V_{2}=V_{1} / 2$ and in the subspace $S_{\text {total }}^{z}=0$. The computation of the spectrum was performed using an existing implementation of an exact diagonalization by Jörg Schulenburg [Sch].

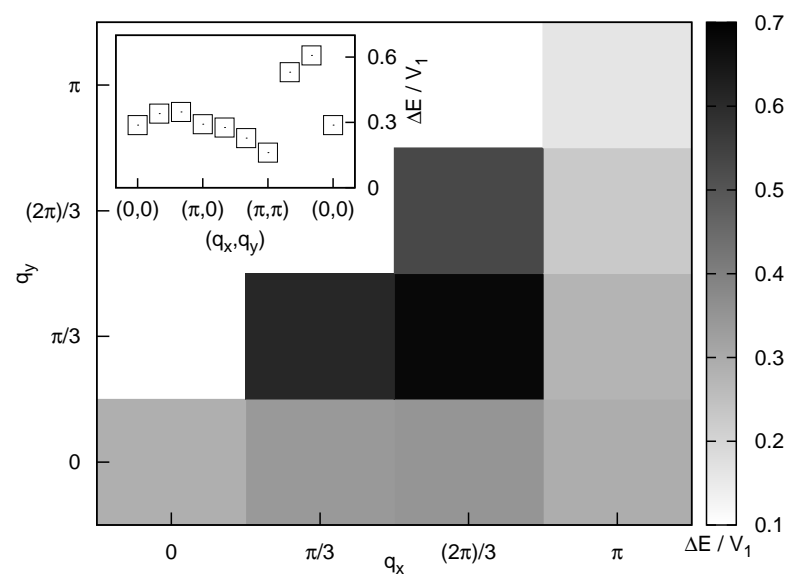

figure 7.12: (Published in [KHFP11a].) Exact diagonalization data for a $6 \times 6$ lattice with periodic boundary conditions at $t_{i}=-0.1 V_{i}$ and $V_{2}=V_{1} / 2$. Shown are the energy differences $\Delta E=E_{q}-E_{0}$ for different $q$-values (only $q_{y} \leq q_{x}$ ) in a gray scale $\left(\Delta E_{\min }(\pi, \pi)=0.1609 V_{1}\right)$. The spectrum shows no tendency for degeneracy and therefore a topologically ordered state is unlikely. In the inset the same values are shown along the path $(0,0) \rightarrow(\pi, 0) \rightarrow(\pi, \pi) \rightarrow(0,0)$.

The lowest eigenvalues in the different $\mathbf{q}$ sectors of the $S_{\text {total }}^{z}=0$ subspace were calculated and the energy differences to the lowest eigenvalue $E_{0}(0,0)=-10.2854 V_{1}$ are

\footnotetext{
${ }^{3}$ The original version of this paragraph in [KHFP11a] was revised and partially rewritten by Andreas Honecker.
} 
shown in figure 7.12. For symmetry reasons, it is sufficient to concentrate on the region $0 \leq q_{x} \leq \pi, 0 \leq q_{y} \leq q_{x}$ of the Brillouin zone. The main panel of figure 7.12 shows a gray scale plot of the energy differences in this region, the inset a different representation of essentially the same data. A minimal energy gap $\Delta E_{\min }=0.1609 V_{1}$ is obtained for $\mathbf{q}=(\pi, \pi)$ above a unique ground state on the $6 \times 6$ lattice. This is comparable to the dispersion along the $q_{x}$ direction, where there is a maximum gap $\Delta E_{\max }(2 \pi / 3,0)=0.3482 V_{1}$. Accordingly, the large value of $\Delta E_{\min }$ is interpreted as evidence against a ground-state degeneracy. An additional check of the lowest eigenvalues in different spin sectors yields no further information as all resulting energy differences are higher than the largest gap $\Delta E_{\max }$.

The spectrum shown in the inset in figure 7.12 is also qualitatively different from the spectrum of the Heisenberg model (see, e.g., references [Maj10, RSH04]). Indeed, in the latter case one would expect a behavior close to $q_{x}=0, q_{y}=0$ which is similar to the vicinity of the ordering wave vector (which is $q_{x}=\pi, q_{y}=\pi$ for the Néel state). The excitation spectrum shown in figure 7.12 is therefore consistent with the absence of classical order, as demonstrated by the quantum Monte-Carlo simulations.

While it would be desirable to perform a finite-size analysis, the $6 \times 6$ lattice was selected as the only accessible lattice which has the symmetries of the infinite system and is compatible with the expected ordered states (the $4 \times 4$ 'square lattice' is not necessarily representative for two dimensions since it can also be interpreted as a four-dimensional torus and was therefore not analyzed). Still, the gap $\Delta E_{\min }$ is comparable to the dispersion of the excited states even on the $6 \times 6$ lattice which suggests that the gap will also stay finite in the thermodynamic limit. As a result, no ground-state degeneracy and for this reason no topological order, is present.

\section{\begin{tabular}{l|l} 
summary & 7.4
\end{tabular}}

The ground-state phase diagram of an anisotropic Heisenberg model with competing interactions on the square lattice was investigated. Extensive quantum Monte-Carlo simulations were performed and in the limit of vanishing quantum fluctuations perturbation theory was applied on the classical antiferromagnetic ground states - Néel and collinear ordered configurations. These configurations were found to be stable under the influence of small fluctuations. A direct phase boundary exists for non-zero fluctuations which was estimated by means of series expansion. In the limit of strong fluctuations a phase of ferromagnetic order in the $x y$ plane was verified which corresponds to a superfluid phase in the equivalent model of hard-core bosons. The signal of the appropriate order parameter was even stronger than for the pure $x y$ model.

In the vicinity of the critical point (where frustration is strongest) and for intermediate values of the spin exchange, a region without magnetic order was found. Motivated by earlier work on similar models $\left[\mathrm{BBB}^{+} 05\right]$ the correlations of dimers on nearest-neighbor bonds was calculated and no long-range order could be detected. Additionally, the lowenergy spectrum was computed by exact diagonalization on a small finite periodic lattice for parameters from the disordered region and no ground-state degeneracy was found. Thus, all evidence for the anisotropic frustrated spin-1/2 Heisenberg model on the square lattice indicates a quantum disordered ground state without topological order. 


\section{anisotropic frustrated Heisenberg model on the honeycomb lattice}

The content of this chapter is published as a regular article in Physical Review B $\left[\mathrm{KAC}^{+} 12\right]$. The presented work was a collaboration with Andreas Honecker (Göttingen) and Marcelo Arlego, Daniel Cabra and Gerardo Rossini from the University of La Plata (Argentina). The project was initiated by the group from La Plata who contributed the linear spinwave calculations, and calculated Padé approximations for higher-order series expansion results from Oitmaa and Singh [OS11]; the fourth order series expansions were performed by Andreas Honecker. The quantum Monte-Carlo and exact-diagonalization results were computed by me.

It may be that some parts of the following chapter resemble the manuscript of reference $\left[\mathrm{KAC}^{+} 12\right]$. The manuscript was written mainly by me apart from the methodological introduction to linear spin waves and series expansion, and revisions to all sections by all coauthors.

\section{abstract}

Similar to the last chapter, the phase diagram of a frustrated spin model with anisotropic interactions is analyzed on the honeycomb lattice. The ground-state phase diagram is analyzed by means of quantum Monte-Carlo simulations and the results are compared to the findings from different methods: linear spin-wave calculations in the limit of small and large quantum fluctuations, and series-expansion computations for two different Ising limits of the frustrated model. The investigation of a remaining disordered region is again performed by applying Monte-Carlo simulations and exact diagonalization.

The model was defined in equation (2.23) in its bosonic form, but in the following the anisotropic spin-1/2 interpretation will be used:

$$
H=\sum_{r=1}^{3} \sum_{i, j}\left[t_{r}\left(S_{i}^{+} S_{j}^{-}+S_{i}^{-} S_{j}^{+}\right)+V_{r} S_{i}^{z} S_{j}^{z}\right] .
$$

The index $r=1,2,3$ represents the nearest, next-nearest and third-nearest neighbor connections of a periodic honeycomb lattice with $N=2 \times(L \times L)$ sites. All exchange parameters $t_{r}<0$ are chosen ferromagnetic whereas the $V_{r}$ are positive and, hence, frustration is introduced by the next-nearest neighbor $S^{z}$ interaction. In the present work the parameter space is reduced to $V=V_{2} / V_{1}=V_{3} / V_{1}$ and $t=t_{r} / V_{r}$ which allows for two antiferromagnetic ground states in the classical limit $t=0$ - again a Néel and collinear configuration. The critical point is given for $V=1 / 2$ and the expected phase diagram is similar to the square-lattice case from the previous chapter.

For the ferromagnetic phase, which is expected to be stable for large $\left|t_{r}\right|$, an in-plane ferromagnetic product-wave function yields the energy:

$$
E_{\text {ferro }} / V_{1}=\frac{3}{4} t(1+3 V) N
$$


Furthermore, an intermediate regime without magnetic order is obtained as was the case for the square lattice.

\section{methods}

To determine the phase diagram in the $V-t$ plane, and to analyze the intermediate region of the phase diagram, primarily quantum Monte-Carlo simulations were performed. In the limit of small quantum fluctuations, i.e., in the Ising limit additional series expansion results are presented and will be compared to linear spin-wave results (confer for details [HP40, IS04]) which were calculated by the group in La Plata. The same method is also applied in the ferromagnetic limit and stability arguments provide good estimates of the phase boundaries. Series expansion results were analytically computed in the context of $\left[\mathrm{KAC}^{+} 12\right]$ up to fourth order and numerically up to eighth order in a recent work by Oitmaa and Singh [OS11]. The low-energy spectrum is only accessible by exact diagonalization and yields an insight into the physics of an unidentified ground state in the phase diagram - similar to the square lattice.

\section{Ising limit $\mid 8.1$}

The Ising limit is given by setting all quantum fluctuations $t_{r}=0$ and exhibits two antiferromagnetic ground states for $V_{2}=V_{3}$, as described in section 2.3. Even for small fluctuations the quantum mechanical ground states are expected to consist of these classical states plus some quantum fluctuations that reduce the overall energy and order parameters.

A comparison for the energy per site from quantum Monte-Carlo simulation with perturbative results from series expansion and linear spin wave approximations is given in figure 8.1 for quantum fluctuations governed by $t=-0.05$ and $t=-0.10$. The agreement for small $V<0.45$ and large $V>0.7$ is very good and only in the intermediate regime can discrepancies be observed; these will be discussed below.

Both methods - series expansion and linear spin waves - assume the classical order (Néel or collinear state) as the underlying configuration which is only weakly perturbed by quantum fluctuations. For the series expansion two different calculations up to fourth order $\left[\mathrm{KAC}^{+} 12\right]$ and up to eighth order at discrete points (Oitmaa and Singh [OS11]) are shown in figure 8.1. Both expansions agree very well away from the critical point

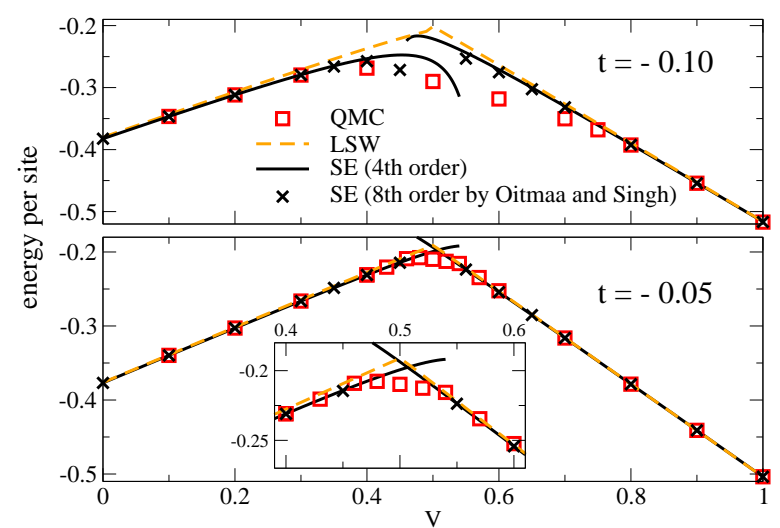

figure 8.1: (Published in $\left.\left[\mathrm{KAC}^{+} 12\right].\right)$ Comparison of energies calculated from different methods at small quantum fluctuations $t=-0.10$ (top) and $t=-0.05$ (bottom): quantum Monte-Carlo (QMC), series expansion (SE, see text also) and linear spin waves (LSW). In the direct vicinity of the critical point $V \approx 1 / 2$ both series expansions become rather unreliable due to an increasing number of divergences. The linear spin wave results underestimate the influence of quantum fluctuations, as can be observed for larger $|t|$. 
$V=1 / 2$. However, the corrections to the classical energies include an increasing number of divergences for higher orders and, thus, become unreliable for larger $|t|$ and $V \nearrow 1 / 2$ (Néel case) or $V \searrow 1 / 2$ (collinear case), respectively. From the fourth-order series results, which are available in analytic form (see supplemental material of $\left.\left[\mathrm{KAC}^{+} 12\right]\right)$ one can derive the direct transition between the two antiferromagnetic states for small $t \neq 0$. The energies are expanded linearly at $V=1 / 2$ and yield a transition line

$$
V(t)=\frac{1278676 t^{4}-69750 t^{3}+6300 t^{2}-225}{2665577 t^{4}-148050 t^{3}+13950 t^{2}-450}
$$

which is shown in figure 8.2 as the blue line. Furthermore, from the series given in [OS11] for the order parameters an estimate of the upper phase boundaries is obtained by applying Padé approximations [GG74]. The corresponding error bars are confidence limits obtained by considering the dispersion of predicted critical points for different Padé approximations.

The linear spin-wave expansions were calculated on a finite lattice with $2 \cdot 10^{4}$ sites around the classical phases. They are stable for $-t<\frac{1-V}{1+3 V}$ (the Néel phase) and $-t<$

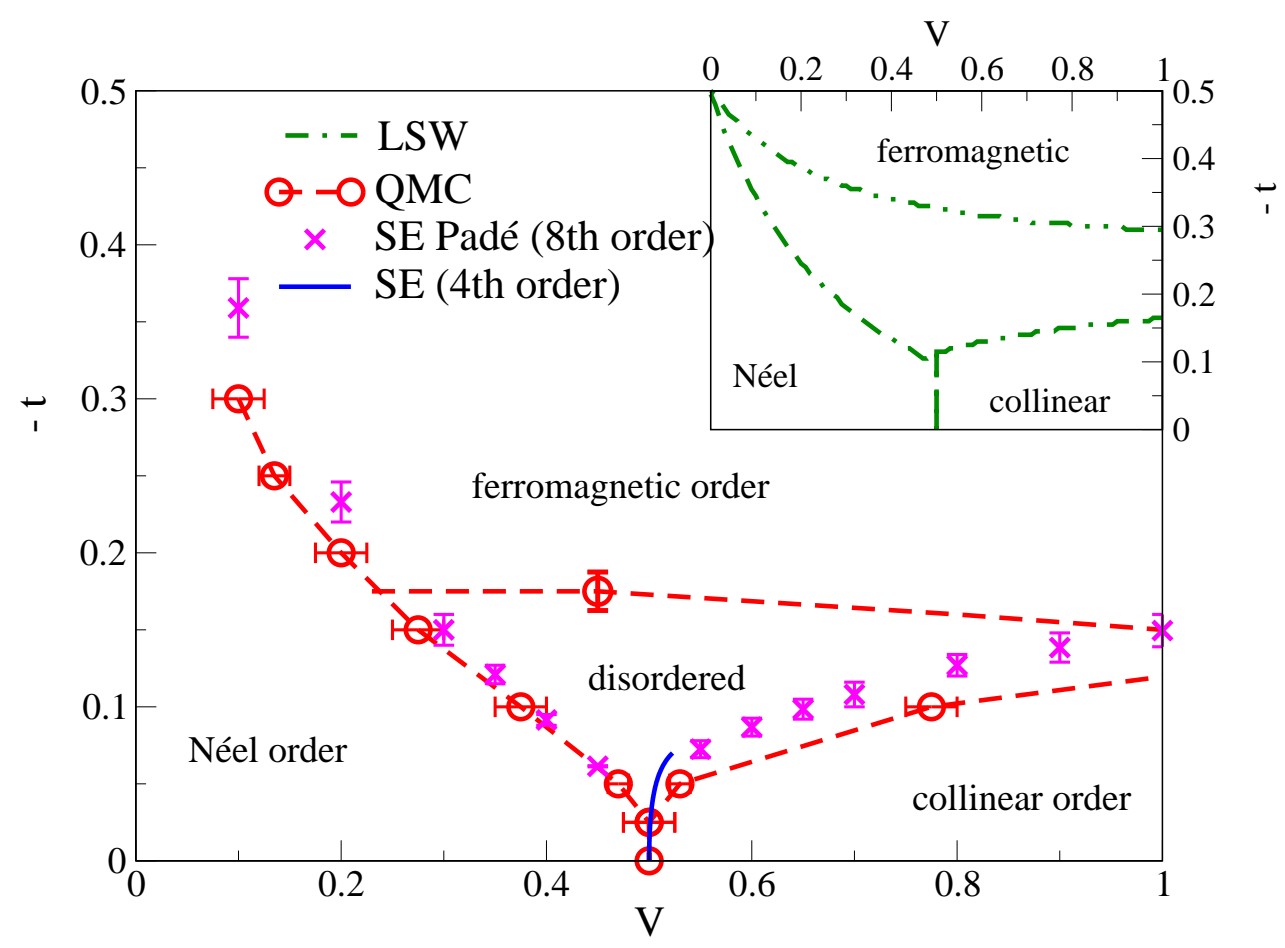

figure 8.2: (Published in $\left[\mathrm{KAC}^{+} 12\right]$.) Ground-state phase diagram for the anisotropic Heisenberg model on the honeycomb lattice. The Ising ground states survive and are separated by a direct first-order transition for small fluctuations $|t|$. Only for values of $|t|>0.175(25)$ can ferromagnetic order in the $x y$ plane be detected. The full blue line represents the first-order transition line between the antiferromagnetic states (equation (8.3)), determined from fourth-order series expansion calculations (SE). Magenta crosses represent phase boundaries determined by the condition of vanishing order parameters, provided by eighth order series expansions [OS11] (see text for more details). The Monte-Carlo results (QMC, red circles and interpolation thereof the dashed red line), where order parameters are computed, provide a very similar phase diagram. The linear spin waves (LSW, green dash-dotted lines in the inset) yield phase transition lines from stability arguments and from the comparison of energies between antiferromagnetic states. 
$-\frac{1-5 V}{1+11 V}$ (the collinear phase). Hence, a direct transition is given at $V \approx 1 / 2$ by comparing energies and the transition is almost independent of $t$. The three approximate phase boundaries are plotted in the inset of figure 8.2.

For the Monte-Carlo simulations no assumptions for the ground state are necessary apart from computing the appropriate order parameters. To identify the regions which show antiferromagnetic order, the structure factors for the Néel and collinear configuration are calculated. The wave vector is given by $\mathbf{q}=(0,0)$ with antiparallel spins on the $A$ and $B$ sites of the unit cell for the Néel state, i.e., each sublattice is ferromagnetically ordered but they are aligned anti-parallel to each other. The collinear state is six-fold degenerate with three wave vectors: $\mathbf{q}=\frac{\pi}{\sqrt{3}}(\sqrt{3}, 1)$ and $\mathbf{q}=\frac{\pi}{\sqrt{3}}(\sqrt{3},-1)$ with $A$ and $B$ parallel, and $\mathbf{q}=\frac{2 \pi}{\sqrt{3}}(0,1)$ with $A$ and $B$ anti-parallel. Additionally all spins can be flipped in the ordered states giving an additional two-fold degeneracy. An example of the temperature-dependent behavior of the order parameter is shown in figure 8.3a for the Néel state. The energies and order parameters are well saturated for different lattice sizes at $T<0.1 V_{1}$.

As expected from the series expansions for small $|t|$, a direct transition between both antiferromagnetic states is obtained. This is probably of first order as the states exhibit different symmetries (figure 8.2). This transition line splits into two for a small value of $0.025<|t|<0.05$ and a new ground state emerges. This critical value of $|t|$ is smaller than for the square lattice (see figure 7.3 in the previous chapter). This is in agreement with the fourth-order series expansion results. By comparing the slopes of the transition lines $V(t)$ given in equations (7.3) and (8.3) graphically in the $V-t$ plane, the steeper slope for the square lattice suggests a larger stability range for the direct transition.

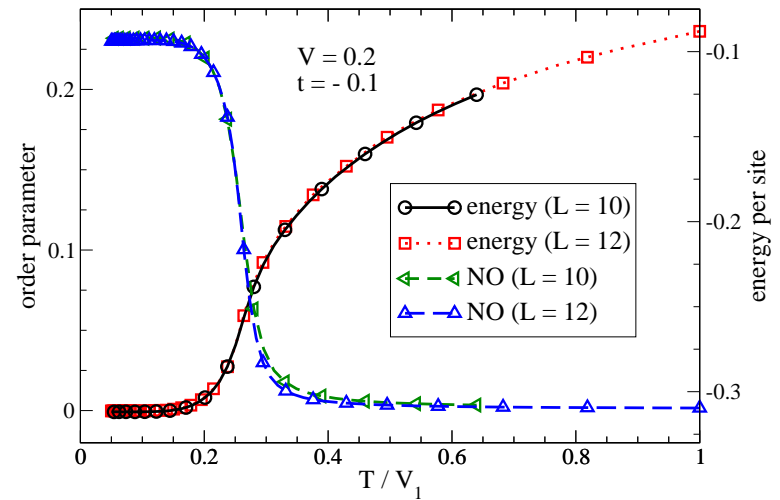

(a) Néel order at $V=0.2$ and $t=-0.1$

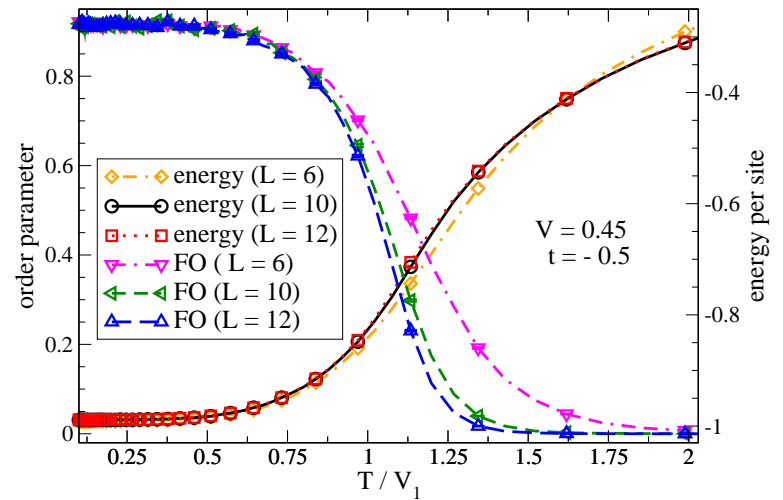

(b) ferromagnetic order at $V=0.45$ and $t=$ $-0.5$

figure 8.3: (Published in $\left[\mathrm{KAC}^{+} 12\right]$.) Monte-Carlo results for the evolution of energies and magnetic order parameters are shown for decreasing temperatures in two different points of the phase diagram, exhibiting Néel order (NO, left) and ferromagnetic order (FO, right).

\section{ferromagnetic limit $\mid 8.2$}

In the opposite limiting case with large $|t|$, the structure of the ground state is not as simple as for the Ising limit. The starting configuration for the approximate linear spin waves will only be a variational ansatz (a ferromagnetic $S^{x}$ product wave function with energy given in equation (8.2)). 


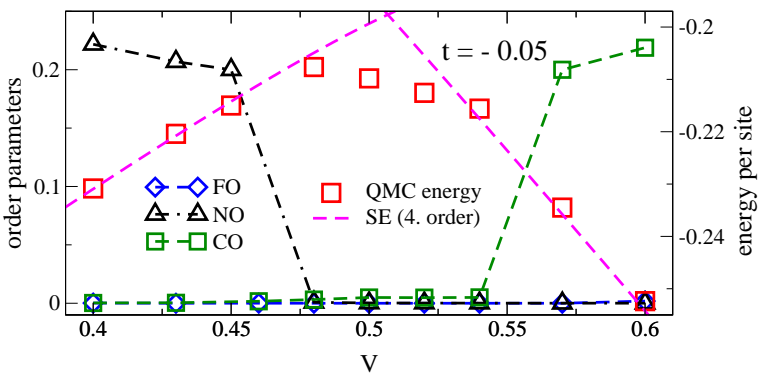

(a) fixed $t=-0.05$ and varying frustration $V$, $L=12$

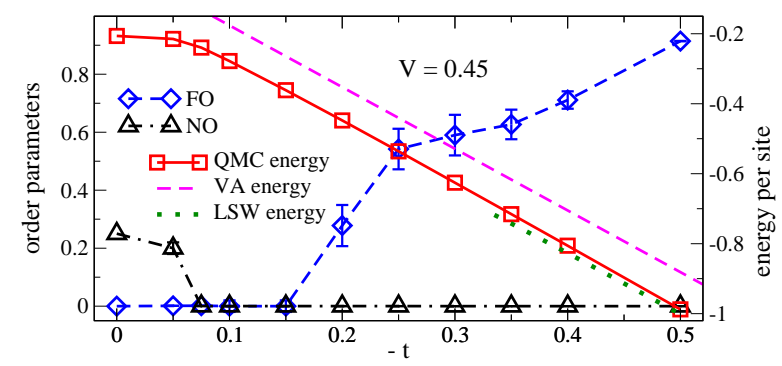

(b) fixed $V=0.45$ and varying fluctuations $t$, $L=12$

figure 8.4: (Published in $\left.\left[\mathrm{KAC}^{+} 12\right].\right)$ Energy and order parameters (FO: ferromagnetic inplane order, NO: Néel order, CO: collinear order) are shown for a horizontal cut (a) through the phase diagram (figure 8.2) at $t=-0.05$ where classical order is absent for $0.48 \lesssim V \lesssim 0.54$, and a vertical cut (b) at $V=0.45$ where there is no finite order parameter for $0.075 \lesssim-t \lesssim 0.15$.

The appropriate order parameter for the ferromagnetic state in the quantum MonteCarlo simulations is the same as for the square lattice and the spin stiffness can be estimated from the winding number. An example of the convergence of energy and order parameter is shown in figure $8.3 \mathrm{~b}$. Careful calculations of the order parameter for the nonantiferromagnetically ordered regions of the phase diagram show a non-vanishing signal only for $|t|>0.15$. Two different parameter scans are shown in figure 8.4. In the left panel order parameters and energies from Monte-Carlo simulations and series expansions are shown for $t=-0.05$ and varying frustration $V$ from Néel to collinear behavior; a finite region without any magnetic order is identified, which also explains the discrepancy of the energies from quantum Monte-Carlo and series expansions shown here and in figure 8.1. In the right panel a similar scan is presented, here for fixed $V=0.45$ and $t$ varying from Néel to ferromagnetic behavior, where the Monte-Carlo energy is compared with the classical variational ansatz [equation (8.2)] and linear spin-wave calculations. The agreement between the Monte-Carlo and spin-wave energies is remarkably good.

The resulting phase diagram is given in figure 8.2 and looks very similar to the result of the previous chapter for the square lattice. However, the region without magnetic order is different on a quantitative level and the classical antiferromagnetic states are only stable for smaller values of $t$.

\section{\begin{tabular}{l|l} 
intermediate regime & 8.3
\end{tabular}}

As for the square lattice, the finite-size effects in the intermediate regime are very strong and Monte-Carlo simulations on small lattices indicate ferromagnetic order. However, for low temperatures and larger lattices $(L>12)$ the signal disappears. Thus, the estimation of the second critical value for $t(V)$ - at which ferromagnetic order arises - is rather difficult and only calculated at the point $V=0.45$. 


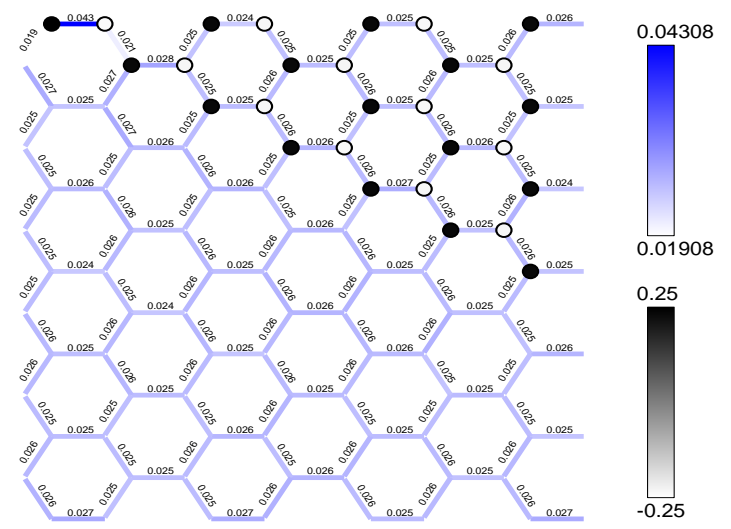

(a) Néel state at $V=0.2$ and $t=-0.1$ ( $T=$ $0.05 J)$

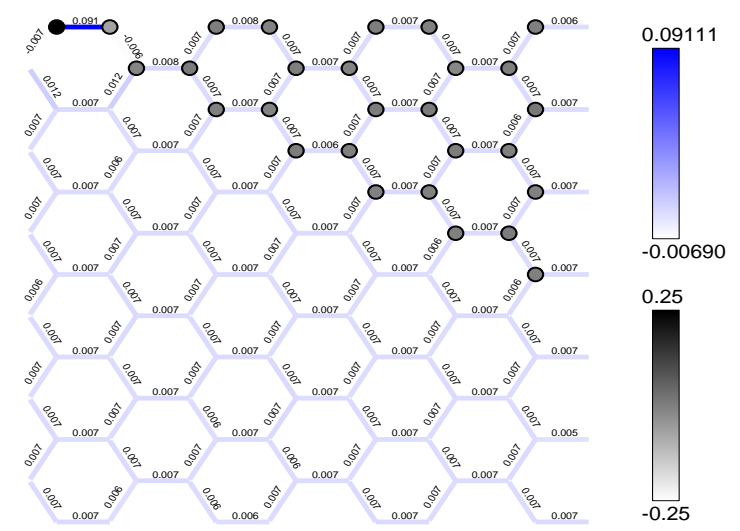

(c) disordered state at $V=0.45$ and $t=-0.1$ $(T=0.02 J)$

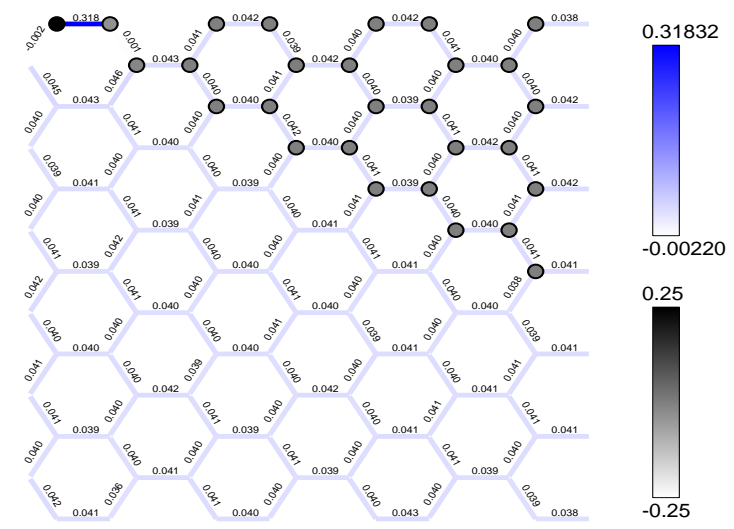

(b) ferromagnetic state at $V=0.45$ and $t=$ $-0.5(T=0.1 J)$

figure 8.5: (Published in $\left.\left[\mathrm{KAC}^{+} 12\right].\right)$ Quantum Monte-Carlo results: each bond (and site) represents the strength of the correlation of a dimer (or spin) at the corresponding distance to the top left dimer (or spin) in blue (dimers, top) or gray (spins, bottom) scales, respectively.

\section{quantum correlations}

Further quantum Monte-Carlo simulations were performed to check for long-range dimer order. Such configurations were reported in earlier works in particular for the honeycomb lattice ,e.g., in $\left[\mathrm{ASH}^{+} 11\right.$, e.g.]. A normalized version of the four point correlator

$$
\left\langle\mathbf{S}_{i} \cdot \mathbf{S}_{j} \mathbf{S}_{k} \cdot \mathbf{S}_{l}\right\rangle-\left\langle\mathbf{S}_{i} \cdot \mathbf{S}_{j}\right\rangle\left\langle\mathbf{S}_{k} \cdot \mathbf{S}_{l}\right\rangle
$$

was calculated. The indices $i \& j$, and $k \& l$, refer to sites at two separate nearest-neighbor bonds.

In figure 8.5 these correlations are presented on a representative lattice where the strength of the correlation is given in a color code (blue scale, top) and distances of the two bonds $i-j$ and $k-l$ are given by the distance between each bond to the top left reference bond. In addition, the $S^{z}$ correlation functions are shown in gray scale (bottom scale) on the sites with respect to the top left site of the lattice. Three different plots are given: as a reference figure 8.5a shows a Néel-ordered configuration where the values of the $S^{z}$ correlations oscillate for different sublattices and show a constant nearly maximal amplitude. The dimer correlations are small and show no sign of ordering. For parameters inside the in-plane ferromagnetic region (figure 8.5b) in either the spin or the dimer correlations no signature can be detected, i.e., spin correlations drop rapidly to zero and dimer correlations adopt a constant distance-independent value. The same applies for the disordered region (figure 8.5c) and only a minor detail distinguishes the two 


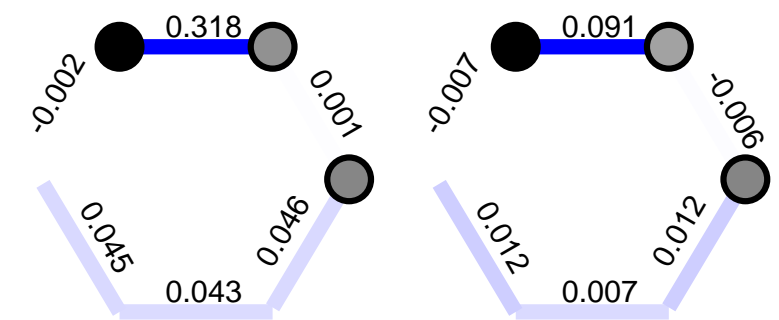

(a) ferromagnetic $(t=(\mathrm{b})$ disordered $(t=$ $-0.5)$ $-0.1)$ figure 8.6: (Published in $\left.\left[\mathrm{KAC}^{+} 12\right].\right)$ The direct comparison of dimer correlations - in an enlarged illustration of the top left hexagon of figure $8.5 \mathrm{~b}$ and $\mathrm{c}$ - shows a small enhancement of correlations on two bonds for the disordered phase $(V=0.45$, original lattice $L=12$ ). The upper bond is again the reference bond for all dimer correlations.

calculations: apart from the different scales (see numbers at the upper (blue) scale) which are explained by the different strength of the quantum correlations $(t=-0.1,-0.5)$, there is a small discrepancy in the relative values of the dimer correlations inside the top left hexagon. To clarify this statement figure 8.6 shows only these top left hexagons of figures $8.5 \mathrm{~b}$ and $8.5 \mathrm{c}$. A small enhancement of the dimer correlations on the two bonds neighboring the opposite bond of the reference bond (top) compared to the correlation on the opposite bond itself is observed. Thus, an extremely short-ranged ordering of dimers is observed in the disordered phase, which is absent in the ferromagnetic state.

\section{low-energy spectrum}

The calculation of the low-energy spectrum is performed by using exact diagonalization with the code package of Jörg Schulenburg [Sch]. For parameters $V=0.45$ and $t=-0.1$, which mark a point inside the disordered region of the phase diagram, the lowest eigenvalues are calculated in the $S_{\text {total }}^{z}=0$ spin sector for several lattices $(N=18,24,28,32,34)$ with periodic boundary conditions. The spectrum of energy differences $\Delta E_{\mathbf{k}}=E_{\mathbf{k}}-E_{0}$ for the largest lattice is shown in figure 8.7. This measures the gap from the ground state energy $E_{0}$ (which belongs to the $\mathbf{q}=0$ subspace and its scaling is shown in the upper panel of figure 8.8) to the lowest eigenvalues $E_{k}$ in the different $\mathbf{q} \neq 0$ sectors and to the first excited state in the $\mathbf{q}=0$ subspace. As for the square lattice the smallest gap is found in the $\mathbf{q}$ subspace of the Néel ordering wave vector (square lattice $\mathbf{q}_{\text {Néel }}=(\pi, \pi)$, honeycomb lattice $\left.\mathbf{q}_{\text {Néel }}=(0,0)\right)$. This gap stays finite in both cases as shown in figure 8.8 (middle, AF gap) for the honeycomb lattice.

However, a calculation of the lowest eigenvalues for higher $S_{\text {total }}^{z}$ subspaces reveals that the gap between $E_{0}$ and the lowest eigenvalue in the $S_{\text {total }}^{z}=1$ subspace vanishes in a

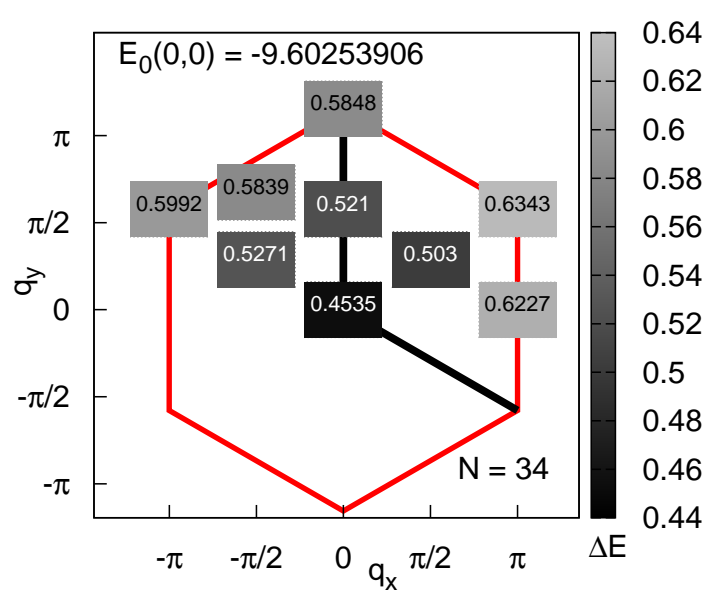

figure 8.7: (Published in $\left.\left[\mathrm{KAC}^{+} 12\right].\right)$ Spectrum of energy gaps $\Delta E_{k}=E_{k}-E_{0}$ for the $S_{\text {total }}^{z}=0$ subspace for $N=34$ at $V=0.45$ and $t=-0.1$. The smallest gap at $\mathbf{q}=0$ is stable in a finite-size analysis (see figure 8.8 middle). 
finite-size scaling for system sizes $N=18$ to $N=34$ (plotted in the lower panel of figure 8.8). In the square lattice this energy difference was larger than the gaps in the $S_{\text {total }}^{z}=0$ subspace. This ferromagnetic correlation in the honeycomb lattice, which is also observed in the correlation functions of the $S^{x y}$ components in the exact-diagonalization results, was also observed in the quantum Monte-Carlo simulations on small lattices but for larger lattices ferromagnetic order was ruled out. This emphasizes that the finite-size effects in the disordered state are stronger on the honeycomb lattice that on the square lattice.
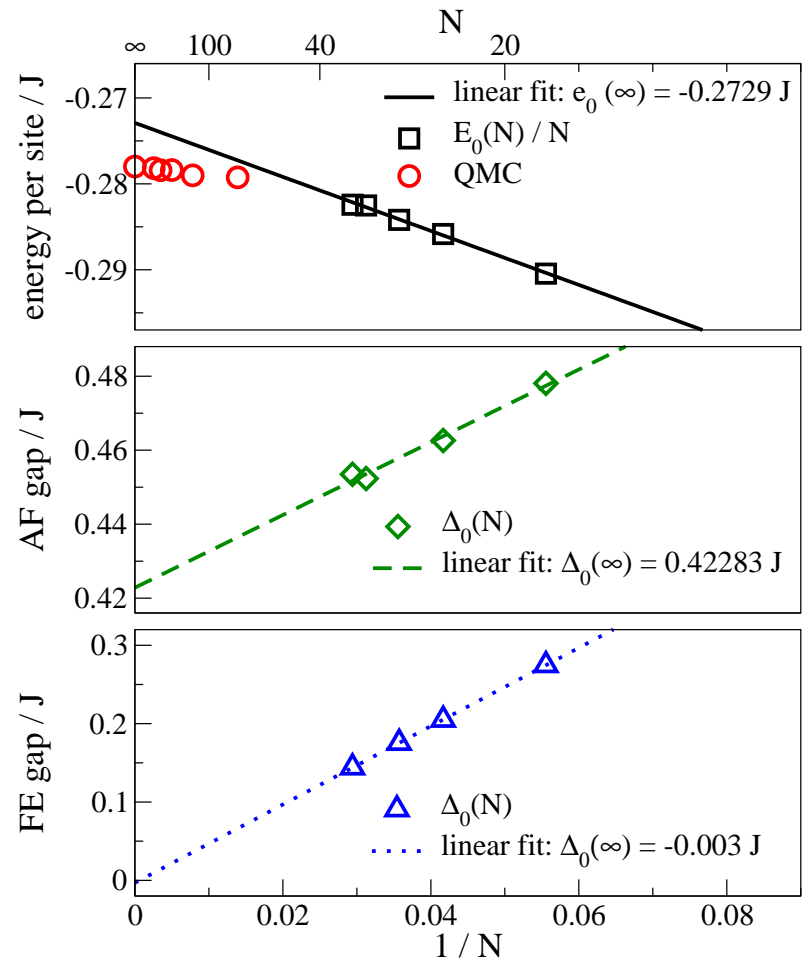

figure 8.8: (Published in $\left.\left[\mathrm{KAC}^{+} 12\right].\right)$ Scaling of the ground state energy and two gaps with the system size $N$ from exact diagonalization at $V=0.45$ and $t=-0.1$. The antiferromagnetic gap (AF) inside the $S_{\text {total }}^{z}=0$ subspace stays finite. However, the ferromagnetic (FE) gap between the ground state energy and the lowest eigenvalue of the $S_{\text {total }}^{z}=1$ subspace scales to zero.

Nevertheless, for the present model a topologically ordered state can be excluded in the disordered phase because of the clearly finite antiferromagnetic gap which was found by exact diagonalization. For a topologically ordered state a four-fold groundstate degeneracy is expected in the $S_{\text {total }}^{z}=0$ subspace for a spin- $1 / 2$ model an a twodimensional periodic lattice with an aspect ratio close to one, i.e. on a torus with similar circumferences in both directions [MLMS02].

\section{\begin{tabular}{l|l} 
& \\
summary & 8.4
\end{tabular}}

The phase diagram for an anisotropic Heisenberg model on the honeycomb lattice with up to third-nearest neighbor interactions was calculated by means of quantum MonteCarlo simulations and compared to results from perturbative methods (series expansions and linear spin-wave approximations). The phase boundaries of three predicted magnetic phases - Néel and collinear phase in the Ising limit and in-plane ferromagnetic order for large quantum fluctuations - were computed. The agreement of the Monte-Carlo results with spin-wave approximations is rather good. In the Ising limit, in addition, the results from series expansion up to fourth order (analytically) and up to eighth order (numerically, by Oitmaa and Singh [OS11]) were compared with quantum Monte-Carlo simulations and the energies and phase boundaries matched very well. 
The Monte-Carlo simulations predict a finite parameter region without any magnetic order in the phase diagram, as was also found for the square lattice system (confer previous chapter). This phase does not show any sign of long-range dimer order and the low-energy spectrum is gapped in the $S_{\text {total }}^{z}=0$ subspace. 


\section{concluding remarks 9}

The investigation of two-dimensional frustrated spin models was motivated by two major topics of modern condensed matter physics: a theoretical explanation for High- $T_{C}$ superconductivity $\left[\mathrm{ZCS}^{+} 06\right]$ and the theoretical design of quantum computation devices [LMSS12]. Although these subjects are very complex and involve a lot of details that go far beyond this work, the better understanding of phase transitions and ground-state phase diagrams in frustrated spin models promotes research in these topics. The present work contributes in particular (i) a thorough analysis of the influence of frustration on the ordering process in the two-dimensional Ising model and (ii) a detailed research of the interplay of frustration and quantum fluctuations for anisotropic quantum spin-1/2 Heisenberg models on the square and honeycomb lattices.

\section{\begin{tabular}{l|l} 
phase transitions in the Ising model & 9.1
\end{tabular}}

In part I of this work the finite-temperature phase transition from the high-temperature paramagnetic phase to an antiferromagnetically ordered state was investigated for frustrated $J_{1}-J_{2}$ Ising models on the square lattice. Methods employed were Monte-Carlo simulations, transfer-matrix calculations and conformal field theory. While controversial predictions about the nature of a particular phase transition were discussed in the literature for the isotropic case (constant homogeneous $J_{2}$ ) [JSS12, KHFP08, KHFP09, LB05, MKT06, MLAGS93], the anisotropic case has attracted fewer attention as the isotropic case [CG05].

\section{isotropic model}

The findings presented in chapter 5 and [KHM11] are summarized in figure 5.11 (see below). The phase transition scenario includes (i) Ising-universal behavior for small couplings $J_{2}<J_{1} / 2$ and at the point $J_{1}=0$, i.e., where two Ising models on sublattices $A$ and $B$ are decoupled, (ii) non-universal continuous phase transitions with varying exponents for dominating next-nearest neighbor coupling $J_{2} \geq J_{1}$ which is originated in an underlying Ashkin-Teller field theory, and (iii) first-order non-critical behavior for the frustrated region $J_{1} \geq J_{2}>J_{1} / 2$. These results stem from an analysis of Monte-Carlo simulations for the lattice model and conformal field theory for the continuous version.

Thus, the presented results satisfy both the old predictions of a continuous phase transition with varying critical exponents (see [LB05]), and the mean-field calculations by Lopéz et al. [MLAGS93] that claim a first-order transition in a finite region of the phase diagram. However, the region of validity of both predictions has changed. In addition, the origin of the non-universal behavior is explained by an underlying AshkinTeller field theory which is known to exhibit varying critical exponents. The exact crossing point between the first-order and non-universal transition could not be derived for two reasons: firstly, the numerical evaluation is hampered by increasing length scales in the 


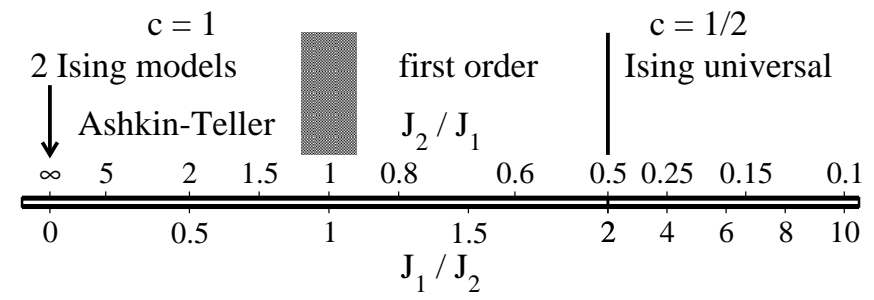

figure 5.11: Phase transition scenario for the frustrated $J_{1^{-}} J_{2}$ Ising model. The transition to the Néel phase for $J_{1}>2 J_{2}$ is Ising universal whereas the transition to the collinear phase shows first-order behavior for $2 J_{2}>J_{1} \gtrsim J_{2}$ and continuous but non-universal behavior for smaller $J_{1}$ and is explained by the underlying Ashkin-Teller field theory. (figure from chapter 5)

vicinity of the crossing point $J_{2} \approx J_{1}$, and secondly, the Ashkin-Teller field theory was derived perturbativly and, hence, the region of its validity can not be accurately estimated. Nevertheless, two scenarios can be proposed: either a level crossing of higher-energy states drives the system from criticality into a non-critical region, or the Ashkin-Teller model reaches a Potts-critical end point which is accompanied by the onset of non-critical behavior [Bax82]. The latter point of view is also adopted heuristically in a recent paper [JSS12] and a critical point at $J_{2}=0.67 J_{1}$ is claimed, in contradiction to the rough estimate of $J_{2} \approx J_{1}$ presented in this work. The result of [JSS12] is derived by comparing universal values of Binder cumulants on small lattices from the different models (frustrated Ising and Potts models). On the one hand the universality of the absolute value of the Binder cumulant is under debate [Sel06], which questions the findings of [JSS12]. On the other hand a scaling invariance of the distance of the two peaks in the histograms, which would strengthen the first-order scenario, is hard to prove for large $J_{2} \geq 0.7 J_{1}$ due to the increasing length scales. The ansatz of numerical finite-size calculations is probably not suitable to solve this conflict.

In conclusion, it is proven that the phase transition from the paramagnetic into the collinear state for the frustrated Ising model in two dimensions exhibits both a nonuniversal critical region and a non-critical region. This result should also be valid if small quantum fluctuations are included. In $\left[\mathrm{RFC}^{+} 04\right]$ anisotropic quantum fluctuations are added to the frustrated Ising model and similarly a phase transition is identified which shows first-order behavior in the vicinity of the critical point $J_{2}=J_{1} / 2$ and turns into a continuous transition for larger couplings $J_{2}$.

\section{anisotropic model}

The spatial anisotropy in the next-nearest neighbor coupling introduces a new ground state for which the two sublattices of the original square lattice exhibit collinear order. For the transition from the paramagnetic phase into this state Chitov and Gros predicted an incommensurate ordering for intermediate temperatures [CG05]. The wave-vector of the structure factor giving a signal for this state depends on the specific value of the isotropic nearest-neighbor interaction.

In chapter 6 this phase transition was analyzed by means of Monte-Carlo simulations. In particular the results for spin-spin correlation functions and the corresponding structure factor prove the existence of such an incommensurate ordering. Moreover, it was shown that the wave vector that describes the phase on finite lattices also depends on the temperature. The structure factor of the finite system shows a signals for a set of wave vectors depending on the nearest-neighbor coupling and the temperature, as shown 


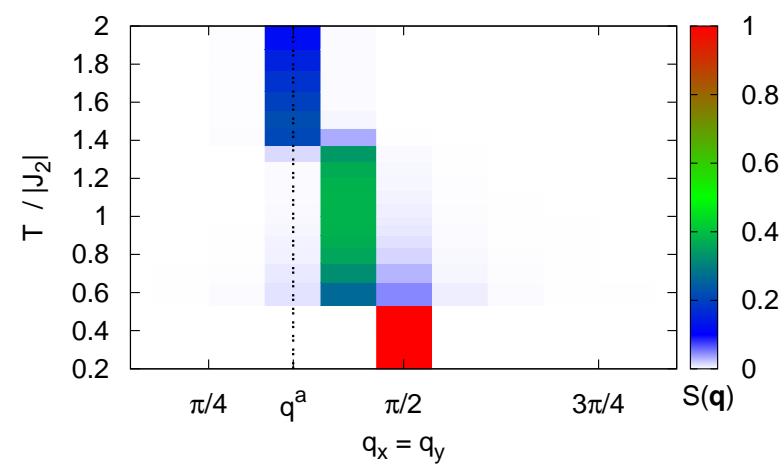

figure 6.10: Structure factor for $q_{x}=q_{y}$ and a lattice of size $L=28$ and nearest-neighbor interactions $J_{1}=-0.867768\left|J_{2}\right|$. A staircase of signals is observed for varying decreasing temperatures and the wave vectors tend towards $(\pi / 2, \pi / 2)$. (figure from chapter 6 )

for example in figure 6.10. The wave vectors with a finite signal in the structure factor vary for decreasing temperatures between a limiting value $\mathbf{q}^{a}$ predicted in [CG05] and the wave vector indicating the ground state $(\pi / 2, \pi / 2)$. The number of signals depend on the system size. Thus, a floating phase with varying order parameter is verified that resembles a devil's staircase [Bak82].

\section{\begin{tabular}{l|l} 
quantum disordered ground states & 9.2
\end{tabular}}

By introducing quantum fluctuations into the frustrated spin models the determination of the ground state phase diagram becomes a challenging task and demands the application of different methods to the classical case. Quantum Monte-Carlo simulations have been performed, which only work for non-frustrating fluctuations. In addition, series expansions and exact diagonalization have been applied in specific regions of the phase diagram. The phase boundaries of three magnetic ground states that are stable in the limit of small and large fluctuations, respectively, were identified. A comparison of both phase diagrams - for the square and honeycomb lattice - reveals that the stability region of the classical antiferromagnetic states is smaller in the honeycomb lattice. Thus, the influence of quantum fluctuations is more important for this lattice, as was assumed in the introduction by arguing that the coordination number is lower for this lattice. In both cases - for the square lattice with couplings $J_{1}$ and $J_{2}$, and for the honeycomb lattice with additional $J_{3}$ - a finite region in the phase diagram was determined for which no classical magnetic order could be found (see figures 7.3 and 8.2).

Motivated by earlier works on similar frustrated quantum spin models on the same lattices $\left[\mathrm{BBB}^{+}\right.$05, OW96, for the square lattice] and [CLR11, FSL01, for the honeycomb lattice], calculations of higher order correlation functions were performed to detect longrange ordered quantum phases, i.e., valence-bond solids. However, no finite signal was observed in any order parameter and, hence, all evidence hint towards a quantum disordered ground state in these anisotropic models. In isotropic models such disordered ground states without any finite order parameter are referred to as spin liquids [Bal10]. The state is described by a superposition of dimer coverings whereas the dimers are given by the $S=0$ spin singlets of two spins, however, for the anisotropic models dimers with lowest energy are given by the $S=1, S^{z}=0$ triplet state. The correlations between spins and dimers are expected to show only an enhanced signal for short distances, this was also obtained in the present anisotropic models. 


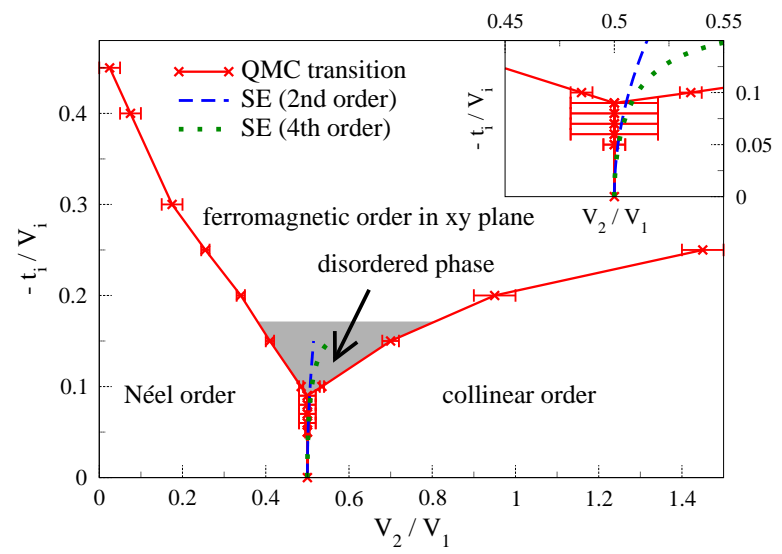

(a) figure 7.3: square lattice

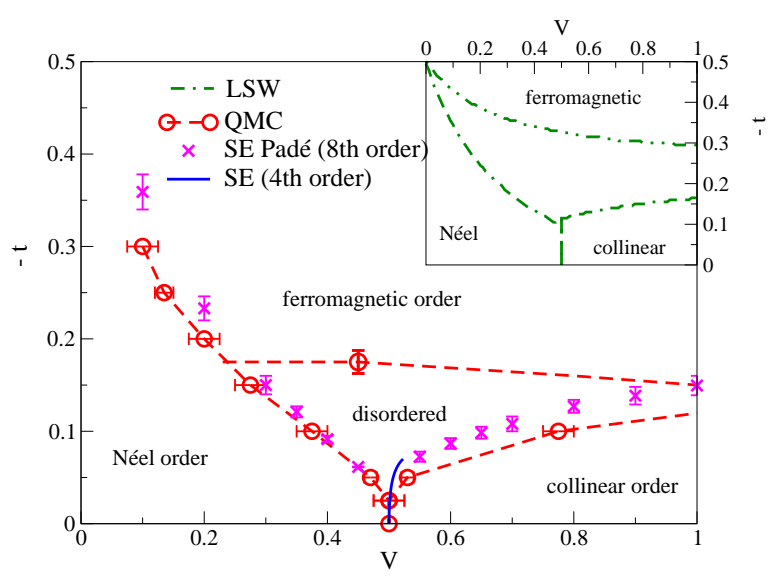

(b) figure 8.2: honeycomb lattice

Ground-state phase diagrams for the anisotropic spin- $1 / 2$ Heisenberg models: (a) on the square lattice with nearest and next-nearest neighbor interaction, and (b) on the honeycomb lattice with additional third-nearest neighbor interactions (quantum fluctuations $t=t_{i} / V_{i}$ over frustration $V=V_{2,3} / V_{1}$ ). (figures from chapters 7 and 8 )

\section{square lattice}

An intermediate disordered phase is discussed for the isotropic $J_{1^{-}} J_{2}$ spin-1/2 Heisenberg model as well [JYB11, RDS ${ }^{+} 10$, WGWV11]. The ground state phase diagram for this model also exhibits the two antiferromagnetic states which are stable in the anisotropic case for small fluctuations. In the vicinity of the critical point $J_{2}=J_{1} / 2$ the order parameters of these phases vanish and a new phase emerges. Thus, the isotropic case shows the same behavior as the model discussed in this thesis at an intermediate value of $t_{i} \approx-0.15 V_{i}$ (see figure 7.3). The characterization of this intermediate phase has been approached by numerous methods: very recently a density-matrix renormalization study [JYB11] classifies the state as a spin liquid by calculating the lowest excitations, and a different tensor-network approach [WGWV11] excludes valence-bond solid order for the questioned region and argues in favor of a spin liquid state by comparing ground-state energies to exact diagonalization results. In an older work by Oitmaa et al. [OW96] a ground-state phase diagram is given by means of series-expansion calculations for all values $0 \leq t \leq 0.5$ and the result is very similar to figure 7.3: for small fluctuations a direct transition between the antiferromagnetic states is given, and for intermediate values of $t$ the transition line splits and a spin liquid emerges which is stable up to $t=0.5$ (the isotropic point).

In the context of spin liquids also the emergence of topological order is discussed. For the anisotropic model analyzed here topological order was excluded by the absence of a degenerate ground state for a periodic lattice with an aspect ratio of one. However, for the isotropic case a study of non-periodic lattices with aspect ratios different from one yielded finite gaps as well but the outcome was interpreted differently, i.e., for a non-periodic asymmetric lattice topological order follows from the finite gaps [JYB11].

\section{honeycomb lattice}

Similarly on the honeycomb lattice the ground-state phase diagram was investigated for the isotropic model. Several quantum ordered and disordered phases were identified in the phase diagram $\left[\mathrm{ASH}^{+} 11, \mathrm{CLR} 11, \mathrm{OS} 11, \mathrm{RAT} 11\right]$. For the case with equal couplings 
$J_{2}=J_{3}$ a quantum disordered intermediate phase is claimed by applying bosonization techniques [CLR11] and series expansion [OS11]. In addition a thorough analysis of the isotropic model is given in [ASH$\left.{ }^{+} 11\right]$ where valence-bonds solids and disordered phases are predicted by applying finite-size scaling to exact diagonalization results on lattices with $N \leq 40$. Thus, the ground-state phase diagram with an intermediate disordered phase calculated in this work for anisotropic Heisenberg coupling fits very well into the overall picture of frustrated spin-1/2 models on the honeycomb lattice.

However, the comparison of exact diagonalization and quantum Monte-Carlo results in chapter 8 has shown that finite-size effects play a crucial role in the vicinity of the critical point. In-plane ferromagnetic order was excluded by Monte-Carlo simulations but gave a finite signal in the exact diagonalization on smaller lattices. Nevertheless, the finite singlet gap which was obtained within exact diagonalization for periodic lattices with an aspect ratio close to one is interpreted as absence of topological order which was not discussed in earlier approaches for the isotropic case.

\section{hard-core bosons}

So far in the numerical analysis of hard-core bosonic models on frustrated lattice geometries an emergence of quantum ordered or disordered phases was not reported. A stable disordered phase was reported previously only for models with intrinsic disorder, i.e, spatially varying chemical potential [BBT02]. For the square lattice with frustrating repulsive interactions [CMWK08, $\mathrm{BS} 00, \mathrm{HBS}^{+} 01, \mathrm{NC0}$ ] the stability of supersolid phases for variable fillings was discussed, however, at half-filling (the case which was studied in chapter 7$)$ the phase diagram of a very similar model $\left(t_{2}=0\right)$ includes no disordered phase [BS00]. For similar studies on the triangular lattice which is also highly frustrated the formation of a stable supersolid phase is reported [HD05, $\left.\mathrm{MPB}^{+} 05, \mathrm{WT} 05\right]$. It is argued that the phase is stabilized by an order-by-disorder mechanism [VBCC80] and, hence, a quantum disordered phase was not observed on the triangular lattice.

Thus, the results from chapters 7 and 8 provided also new insights into the physics of frustrated bosonic models, which may be particularly interesting for possible realizations in optical lattices $\left[\mathrm{LSA}^{+} 07, \mathrm{SOL}^{+} 11\right]$.

\section{\begin{tabular}{l|l} 
future prospects & 9.3
\end{tabular}}

The objective of the present thesis was to provide a better understanding of phase transitions in classical frustrated Ising models and the derivation of ground-state phase diagrams for anisotropic frustrated quantum spin-1/2 models. Both aims were accomplished.

A possibility for a future project is the combination of both topics by analyzing the finite-temperature phase transition into the magnetic states and the quantum phase transitions [Sac99] occurring in the ground-state phase diagram of the quantum models in detail. The transition from the disordered state into the ferromagnetic region of the phase diagram is a promising candidate for continuous quantum phase transition because of the very smooth behavior of the energy and the order parameter at the phase transition which was observed in this work.

Another interesting project would be the analysis of the stability of the disordered phase against a magnetic field or additional ring-exchange terms which are known to strengthen valence-bond solid order $\left[\mathrm{BBB}^{+} 05\right.$, San12, SS02]. 
Finally, to analyze a possible connection of the disordered state with High- $T_{C}$ superconductivity, the model has to be changed by removing spin variables on single sites and allowing for a hopping of the remaining spins onto these holes. This model is referred to as $t-J$ model [And87, EKL90] and enables the dimers which are formed in the disordered phase to propagate through the lattice. 
appendices

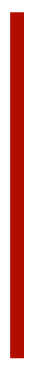





\section{computational details}

Some details on the implementation of the applied numerical methods which have been developed or refined in the context of the present thesis are given here.

\section{classical Monte-Carlo $\mid$ A.1}

The algorithm for the classical Monte-Carlo simulation was implemented by me. Some parts of the program were already implemented during the Diploma thesis [Kal08] and used to perform large-scale Monte-Carlo simulations on the square lattice Ising model.

The program provides an optional parallelization using OpenMP [CDK ${ }^{+} 00, \mathrm{CJv}_{\mathrm{NP}} 07$ ] which distributes simulations at different temperatures to a set of CPUs with a shared memory. An additional MPI [GFB ${ }^{+} 04$, e.g.] parallelization enables the simultaneous computation of independent runs each with its own (independent) random-number generator.

The source code is available at http://www.theorie.physik.uni-goettingen.de/ $\sim$ kalz. The package contains four $\mathrm{C}++$ source files, a readme.txt explaining all (non)optional parameters, and a makefile which has to be adopted to the local computer structure by providing the locations for necessary libraries (boost, MPI, SPRNG).

Different versions of random-number generators are integrated: the basic C-rand() function in the single CPU version, a lagged Fibonacci generator [MCPR95] from the SPRNG library [MS00], and the Mersenne-Twister [MN98] from the Boost library (http://www. boost.org/).

The program is able to simulate the square lattice Ising model with nearest and nextnearest neighbor interactions, a bilayer Ising model and the Ashkin-Teller model. In addition a line update and the exchange Monte-Carlo (or parallel tempering) algorithm are implemented as optional features. The temperature distribution can be chosen linear between the initial and final temperature or logarithmic around a fixed critical temperature with $T_{i}<T_{c}<T_{f}$; also annealing procedure can be included and the temperature steps can be optimized during the thermalization process.

Furthermore, the program contains checkpointing and can store time series of some observables (energy, magnetization, different magnetic order parameters).

The calculation of the complete structure factor is possible but in the basic version only ferromagnetic, Néel and collinear antiferromagnetic order parameters are computed.

A runtime estimation was given in the Diploma thesis [Kal08, appendix A.2].

$$
\begin{array}{l|l}
\text { quantum Monte-Carlo } & \text { A.2 }
\end{array}
$$

For the quantum Monte-Carlo simulations an existing code package from the ALPS library $\left[\mathrm{AAC}^{+} 07, \mathrm{ADG}^{+} 05, \mathrm{BCE}^{+} 11\right]$ was used and improved by integrating an exchange MonteCarlo update. The original packages are available at http://alps.comp-phys.org/, 
along with instructions and tutorials concerning the installation and usage. The ALPS library also includes a class for the evaluation and error estimation of observables.

The application employed in chapters 7 and 8 is based on the implementation of the Stochastic Series Expansion (dirloop_sse_v1) by Alet et al. [AWT05]. The additional exchange Monte-Carlo algorithm was implemented by Sebastian Fuchs and refined by me and is included in a package available at http://www. theorie.physik. uni-goettingen . de $\sim^{\sim} \mathrm{kalz}$. Furthermore, an annealing procedure is implemented which allows for prethermalization before the time-consuming parallel tempering is applied. The content is given by some source files and in addition two files with instructions are included:

- HOWTO: describes how to install the program and how to start a run

- EXAMPLE: gives an exemplary input file

\section{observables}

The package also includes the implementation of the observables employed in chapter 7 and 8 which can be calculated optionally. In detail the following additional (not included in the original version) observables are implemented:

- Néel and collinear order parameter for the square and honeycomb lattice

- spin correlation functions for both systems

- complete structure factor for the square lattice

- spin stiffness measurements (superfluid density)

- local magnetization and bond energy

- bond correlation between nearest-neighbor bonds

\section{\begin{tabular}{l|l} 
transfer-matrix implementation & A.3
\end{tabular}}

The transfer-matrix program is available in two versions: a matrix diagonalization for small systems, and a matrix vector multiplication using a decomposition of the sparse matrix. For the first one a lapack library is necessary and needs to be linked against the program. The package contains the source code and a makefile which perhaps needs to be adapted to the local machine (available at http://www.theorie.physik. uni-goettingen.de/ kalz).

The algorithm is based on the technique presented in reference [dNNS82, confer appendix]. In contrast to a transfer-matrix for the nearest-neighbor square lattice Ising model, for the frustrated case with diagonal couplings the smallest block is not a $2 \times 2$ matrix (connecting two spins from adjacent columns with each other) but a $2 \times 8$ matrix (connecting one with three spins from the neighboring column). Thus, the most efficient implementation which operates with states of size $2^{L}$ ( $L$ is the width of the strip) has to be extended in an intermediate step: the state is enlarged by a factor four (two additional spins are taken into account) and afterwards reduced again by summing up the weights. Therefore, the accessible system sizes are smaller than for the nearest-neighbor model. 
The successive multiplication of the transfer-matrix yields the canonical partition function and, hence, the free energy of the system. The program can calculate the free energy for periodic and antiperiodic boundary conditions, for different temperatures and parameters $\left(J_{1}\right.$ and $\left.J_{2}\right)$ and different lattice sizes (width and length). For large systems a checkpoint is implemented. 


\section{conformal field theory}

Explicit calculations for the conformal field theory part of chapter 5 are presented here.

\section{continuum limit derivations}

The derivation of the continuum limit for the frustrated Ising model is presented using a specific unit cell (shown in figure 5.10) and a continuous representation of the spin variables expanded up to second order around the center of the unit cell. Starting points are the equations (5.5)-(5.7) and the Taylor expansion in (5.8).

unperturbed models

With $\sigma_{I}(x, y)=\sigma_{I}$ and $\partial_{i j}=\partial_{i} \partial_{j}$ the unperturbed Ising models are obtained [equation $(5.10)]$ :

$$
\begin{aligned}
& h_{A}=- \frac{J_{2}}{4} \sigma_{A}\left[\sigma_{A}+a \partial_{x} \sigma_{A}+\frac{a^{2}}{2} \partial_{x x} \sigma_{A}+\sigma_{A}-a \partial_{x} \sigma_{A}+\frac{a^{2}}{2} \partial_{x x} \sigma_{A}\right. \\
&+\left.\sigma_{A}+a \partial_{y} \sigma_{A}+\frac{a^{2}}{2} \partial_{y y} \sigma_{A}+\sigma_{A}-a \partial_{y} \sigma_{A}+\frac{a^{2}}{2} \partial_{y y} \sigma_{A}\right] \\
&=-\frac{J_{2}}{4}\left[4 \sigma_{A} \sigma_{A}+a^{2} \sigma_{A} \partial_{x x} \sigma_{A}+a^{2} \sigma_{A} \partial_{y y} \sigma_{A}\right] \\
& h_{B}=- \frac{J_{2}}{4}\left\{\left[\sigma_{B}+\frac{a}{2} \partial_{x} \sigma_{B}+\frac{a}{2} \partial_{y} \sigma_{B}+\frac{a^{2}}{8}\left(\partial_{x x} \sigma_{B}+\partial_{y y} \sigma_{B}+2 \partial_{x y} \sigma_{B}\right)\right] \times\right. \\
& {\left[\sigma_{B}+\frac{a}{2} \partial_{x} \sigma_{B}-\frac{a}{2} \partial_{y} \sigma_{B}+\frac{a^{2}}{8}\left(\partial_{x x} \sigma_{B}+\partial_{y y} \sigma_{B}-2 \partial_{x y} \sigma_{B}\right)\right.} \\
&\left.+\sigma_{B}-\frac{a}{2} \partial_{x} \sigma_{B}+\frac{a}{2} \partial_{y} \sigma_{B}+\frac{a^{2}}{8}\left(\partial_{x x} \sigma_{B}+\partial_{y y} \sigma_{B}-2 \partial_{x y} \sigma_{B}\right)\right] \\
&+\left[\sigma_{B}-\frac{a}{2} \partial_{x} \sigma_{B}-\frac{a}{2} \partial_{y} \sigma_{B}+\frac{a^{2}}{8}\left(\partial_{x x} \sigma_{B}+\partial_{y y} \sigma_{B}+2 \partial_{x y} \sigma_{B}\right)\right] \times \\
& {\left[\sigma_{B}+\frac{a}{2} \partial_{x} \sigma_{B}-\frac{a}{2} \partial_{y} \sigma_{B}+\frac{a^{2}}{8}\left(\partial_{x x} \sigma_{B}+\partial_{y y} \sigma_{B}-2 \partial_{x y} \sigma_{B}\right)\right.} \\
&\left.\left.+\sigma_{B}-\frac{a}{2} \partial_{x} \sigma_{B}+\frac{a}{2} \partial_{y} \sigma_{B}+\frac{a^{2}}{8}\left(\partial_{x x} \sigma_{B}+\partial_{y y} \sigma_{B}-2 \partial_{x y} \sigma_{B}\right)\right]\right\} \\
&=- \frac{J_{2}}{4}\left\{\left[\sigma_{B}+\frac{a}{2} \partial_{x} \sigma_{B}+\frac{a}{2} \partial_{y} \sigma_{B}+\frac{a^{2}}{8}\left(\partial_{x x} \sigma_{B}+\partial_{y y} \sigma_{B}+2 \partial_{x y} \sigma_{B}\right)\right] \times\right. \\
& {\left[2 \sigma_{B}+\frac{a^{2}}{4}\left(\partial_{x x} \sigma_{B}+\partial_{y y} \sigma_{B}-2 \partial_{x y} \sigma_{B}\right)\right] } \\
&+\left[\sigma_{B}-\frac{a}{2} \partial_{x} \sigma_{B}-\frac{a}{2} \partial_{y} \sigma_{B}+\frac{a^{2}}{8}\left(\partial_{x x} \sigma_{B}+\partial_{y y} \sigma_{B}+2 \partial_{x y} \sigma_{B}\right)\right] \times \\
& {\left.\left[2 \sigma_{B}+\frac{a^{2}}{4}\left(\partial_{x x} \sigma_{B}+\partial_{y y} \sigma_{B}-2 \partial_{x y} \sigma_{B}\right)\right]\right\} } \\
&=- \frac{J_{2}}{4}\left[4 \sigma_{B} \sigma_{B}+a^{2} \sigma_{B} \partial_{x x} \sigma_{B}+a^{2} \sigma_{B} \partial_{y y} \sigma_{B}\right]
\end{aligned}
$$




\section{perturbation term}

For the perturbation terms in equation (5.7) some more calculations are necessary:

$$
\begin{aligned}
& h_{A B}=\frac{J_{1}}{4}\left\{\sigma _ { A } \cdot \left[\sigma_{B}+\frac{a}{2} \partial_{x} \sigma_{B}+\frac{a}{2} \partial_{y} \sigma_{B}+\frac{a^{2}}{8}\left(\partial_{x x} \sigma_{B}+\partial_{y y} \sigma_{B}+2 \partial_{x y} \sigma_{B}\right)\right.\right. \\
& +\sigma_{B}-\frac{a}{2} \partial_{x} \sigma_{B}-\frac{a}{2} \partial_{y} \sigma_{B}+\frac{a^{2}}{8}\left(\partial_{x x} \sigma_{B}+\partial_{y y} \sigma_{B}+2 \partial_{x y} \sigma_{B}\right) \\
& -\sigma_{B}+\frac{a}{2} \partial_{x} \sigma_{B}-\frac{a}{2} \partial_{y} \sigma_{B}-\frac{a^{2}}{8}\left(\partial_{x x} \sigma_{B}+\partial_{y y} \sigma_{B}-2 \partial_{x y} \sigma_{B}\right) \\
& \left.\left.-\sigma_{B}-\frac{a}{2} \partial_{x} \sigma_{B}+\frac{a}{2} \partial_{y} \sigma_{B}-\frac{a^{2}}{8}\left(\partial_{x x} \sigma_{B}+\partial_{y y} \sigma_{B}-2 \partial_{x y} \sigma_{B}\right)\right]\right\} \\
& +\frac{J_{1}}{8}\left\{-\left[\sigma_{B}+\frac{a}{2} \partial_{x} \sigma_{B}+\frac{a}{2} \partial_{y} \sigma_{B}+\frac{a^{2}}{8}\left(\partial_{x x} \sigma_{B}+\partial_{y y} \sigma_{B}+2 \partial_{x y} \sigma_{B}\right)\right] \times\right. \\
& {\left[\sigma_{A}+a \partial_{x} \sigma_{A}+\frac{a^{2}}{2} \partial_{x x} \sigma_{A}+\sigma_{A}+a \partial_{y} \sigma_{A}+\frac{a^{2}}{2} \partial_{y y} \sigma_{A}\right]} \\
& -\left[\sigma_{B}-\frac{a}{2} \partial_{x} \sigma_{B}-\frac{a}{2} \partial_{y} \sigma_{B}+\frac{a^{2}}{8}\left(\partial_{x x} \sigma_{B}+\partial_{y y} \sigma_{B}+2 \partial_{x y} \sigma_{B}\right)\right] \times \\
& {\left[\sigma_{A}-a \partial_{x} \sigma_{A}+\frac{a^{2}}{2} \partial_{x x} \sigma_{A}+\sigma_{A}-a \partial_{y} \sigma_{A}+\frac{a^{2}}{2} \partial_{y y} \sigma_{A}\right]} \\
& +\left[\sigma_{B}+\frac{a}{2} \partial_{x} \sigma_{B}-\frac{a}{2} \partial_{y} \sigma_{B}+\frac{a^{2}}{8}\left(\partial_{x x} \sigma_{B}+\partial_{y y} \sigma_{B}-2 \partial_{x y} \sigma_{B}\right)\right] \times \\
& {\left[\sigma_{A}+a \partial_{x} \sigma_{A}+\frac{a^{2}}{2} \partial_{x x} \sigma_{A}+\sigma_{A}-a \partial_{y} \sigma_{A}+\frac{a^{2}}{2} \partial_{y y} \sigma_{A}\right]} \\
& +\left[\sigma_{B}-\frac{a}{2} \partial_{x} \sigma_{B}+\frac{a}{2} \partial_{y} \sigma_{B}+\frac{a^{2}}{8}\left(\partial_{x x} \sigma_{B}+\partial_{y y} \sigma_{B}-2 \partial_{x y} \sigma_{B}\right)\right] \times \\
& \left.\left[\sigma_{A}-a \partial_{x} \sigma_{A}+\frac{a^{2}}{2} \partial_{x x} \sigma_{A}+\sigma_{A}+a \partial_{y} \sigma_{A}+\frac{a^{2}}{2} \partial_{y y} \sigma_{A}\right]\right\} \\
& =\frac{J_{1}}{4}\left\{a^{2} \sigma_{A} \partial_{x y} \sigma_{B}\right\} \\
& +\frac{J_{1}}{8}\left\{2 \sigma _ { A } \cdot \left[2 \sigma_{B}-2 \sigma_{B}+a \partial_{x} \sigma_{A}-a \partial_{x} \sigma_{B}+a \partial_{y} \sigma_{B}-a \partial_{y} \sigma_{A}\right.\right. \\
& \left.+\frac{a^{2}}{4}\left(\partial_{x x} \sigma_{B}-\partial_{x x} \sigma_{B}+\partial_{y y} \sigma_{B}-\partial_{y y} \sigma_{B}-4 \partial_{x y} \sigma_{B}\right)\right] \\
& +a \partial_{x} \sigma_{A}\left[2 \sigma_{B}-2 \sigma_{B}+a \partial_{x} \sigma_{B}-a \partial_{x} \sigma_{B}-2 a \partial_{y} \sigma_{B}\right. \\
& \left.+\frac{a^{2}}{4}\left(\partial_{x x} \sigma_{B}-\partial_{x x} \sigma_{B}+\partial_{y y} \sigma_{B}-\partial_{y y} \sigma_{B}\right)\right] \\
& +a \partial_{y} \sigma_{A}\left[2 \sigma_{B}-2 \sigma_{B}-2 a \partial_{x} \sigma_{B}+a \partial_{y} \sigma_{B}-a \partial_{x} \sigma_{B}\right. \\
& \left.+\frac{a^{2}}{4}\left(\partial_{x x} \sigma_{B}-\partial_{x x} \sigma_{B}+\partial_{y y} \sigma_{B}-\partial_{y y} \sigma_{B}\right)\right] \\
& +\frac{a^{2}}{2}\left(\partial_{x x} \sigma_{A}+\partial_{y y} \sigma_{A}\right)\left[2 \sigma_{B}-2 \sigma_{B}\right] \\
& =\frac{J_{1}}{4}\left\{a^{2} \sigma_{A} \partial_{x y} \sigma_{B}\right\} \\
& -\frac{J_{1}}{8}\left\{2 a^{2} \sigma_{A} \partial_{x y} \sigma_{B}+2 a^{2} \partial_{x} \sigma_{A} \partial_{y} \sigma_{B}+2 a^{2} \partial_{y} \sigma_{A} \partial_{x} \sigma_{B}\right\} \\
& =-\frac{J_{1}}{4} a^{2}\left(\partial_{x} \sigma_{A} \partial_{y} \sigma_{B}+\partial_{y} \sigma_{A} \partial_{x} \sigma_{B}\right) \text {. }
\end{aligned}
$$

This result is given in equation (5.9). 


\section{complex mapping}

The transformation of $h_{A B}$ from real variables $x$ and $y$ onto the complex plane which is the basis for the conformal operations in two dimensions with variables $z$ and $\bar{z}$ involves

$$
\begin{aligned}
z=x+i y, \quad \bar{z}=x-i y & \wedge \quad x=\frac{1}{2}(z+\bar{z}), \quad y=\frac{1}{2 i}(z-\bar{z}) \\
\partial_{z}=\frac{1}{2}\left(\partial_{x}-i \partial_{y}\right), \quad \partial_{\bar{z}}=\frac{1}{2}\left(\partial_{x}+i \partial_{y}\right) & \wedge \partial_{x}=\left(\partial_{z}+\partial_{\bar{z}}\right), \quad \partial_{y}=i\left(\partial_{z}-\partial_{\bar{z}}\right) \\
d z d \bar{z}=\left|\left(\begin{array}{cc}
1 & 1 \\
i & -i
\end{array}\right)\right| d x d y=2 d x d y & \wedge d x d y=\frac{1}{2} d z d \bar{z}
\end{aligned}
$$

and yields:

$$
\begin{aligned}
h_{A B}= & -\frac{J_{1}}{4} a^{2}\left[\partial_{x} \sigma_{A} \partial_{y} \sigma_{B}+\partial_{y} \sigma_{A} \partial_{x} \sigma_{B}\right] \\
= & -\frac{J_{1}}{4} a^{2}\left[\left(\partial_{z}+\partial_{\bar{z}}\right) \sigma_{A} i\left(\partial_{z}-\partial_{\bar{z}}\right) \sigma_{B}+i\left(\partial_{z}-\partial_{\bar{z}}\right) \sigma_{A}\left(\partial_{z}+\partial_{\bar{z}}\right) \sigma_{B}\right] \\
=- & i \frac{J_{1}}{4} a^{2}\left[\partial_{z} \sigma_{A} \partial_{z} \sigma_{B}+\partial_{\bar{z}} \sigma_{A} \partial_{z} \sigma_{B}-\partial_{z} \sigma_{A} \partial_{\bar{z}} \sigma_{B}-\partial_{\bar{z}} \sigma_{A} \partial_{\bar{z}} \sigma_{B}\right. \\
& \left.+\partial_{z} \sigma_{A} \partial_{z} \sigma_{B}-\partial_{\bar{z}} \sigma_{A} \partial_{z} \sigma_{B}+\partial_{z} \sigma_{A} \partial_{\bar{z}} \sigma_{B}-\partial_{\bar{z}} \sigma_{A} \partial_{\bar{z}} \sigma_{B}\right] \\
= & -i \frac{J_{1}}{2}\left[\partial_{z} \sigma_{A} \partial_{z} \sigma_{B}-\partial_{\bar{z}} \sigma_{A} \partial_{\bar{z}} \sigma_{B}\right] .
\end{aligned}
$$

The total perturbation is then given by:

$$
H_{A B}=\iint d x d y h_{A B}=-i \frac{J_{1}}{4} \iint d z d \bar{z}\left[\partial_{z} \sigma_{A} \partial_{z} \sigma_{B}-\partial_{\bar{z}} \sigma_{A} \partial_{\bar{z}} \sigma_{B}\right]
$$

This result is given in equations (5.12) and (5.13).

\section{gauge invariance}

Here a short comment - without detailed calculations - is given about the choice of the unit cell. The unit cell shown in figure 5.10 is a rather asymmetric choice since the center is given by one of the sublattice sites. The calculations for this unit cell were straightforward and yielded a symmetric set of operators for the perturbation (as seen above). However, a different more symmetric choice was also checked and is shown in figure B.1 below. The two sublattices are treated equally but the result is a set of asymmetric operators

$$
h_{A B} \propto \sigma_{A} \partial_{x y} \sigma_{B}+\sigma_{B} \partial_{x y} \sigma_{A}
$$

However, using partial integration and respecting the periodic boundary conditions for $H_{A B}$, which imply that terms at the boundaries cancel each other out, these operators can be mapped exactly onto the set of operators for the asymmetric unit cell given in equation (B.3).

\section{operator product expansion}

In the following the operator product expansion for the second and third order perturbation will be applied. The rules were outlined in section 4.2 and operators were derived in section 5.2 . 


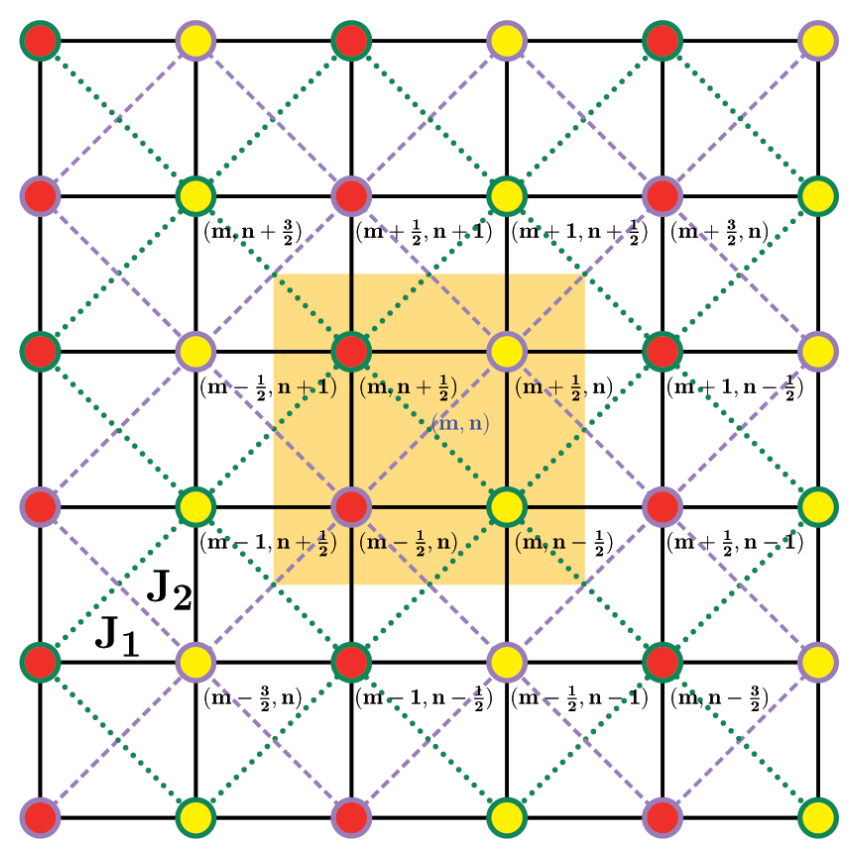

figure B.1: (Prepared by Marion Moliner.) A different more symmetric unit cell for the derivation of the continuum limit for the frustrated Ising model.

second order perturbation

The calculation of the second order terms given in (5.21)-(5.25) is shown for the terms (5.21) and (5.22):

$$
\begin{aligned}
& {\left[\mathcal{O}_{1}(z, \bar{z})-\mathcal{O}_{2}(z, \bar{z})\right]\left[\mathcal{O}_{1}(w, \bar{w})-\mathcal{O}_{2}(w, \bar{w})\right] } \\
= & \mathcal{O}_{1}(z, \bar{z}) \mathcal{O}_{1}(w, \bar{w})-\mathcal{O}_{1}(z, \bar{z}) \mathcal{O}_{2}(w, \bar{w})-\mathcal{O}_{2}(z, \bar{z}) \mathcal{O}_{1}(w, \bar{w})+\mathcal{O}_{2}(z, \bar{z}) \mathcal{O}_{2}(w, \bar{w}) \\
= & \partial_{z} \sigma_{A}(z, \bar{z}) \partial_{z} \sigma_{B}(z, \bar{z}) \partial_{w} \sigma_{A}(w, \bar{w}) \partial_{w} \sigma_{B}(w, \bar{w}) \\
& -\partial_{z} \sigma_{A}(z, \bar{z}) \partial_{z} \sigma_{B}(z, \bar{z}) \partial_{\bar{w}} \sigma_{A}(w, \bar{w}) \partial_{\bar{w}} \sigma_{B}(w, \bar{w}) \\
& -\partial_{\bar{z}} \sigma_{A}(z, \bar{z}) \partial_{\bar{z}} \sigma_{B}(z, \bar{z}) \partial_{w} \sigma_{A}(w, \bar{w}) \partial_{w} \sigma_{B}(w, \bar{w}) \\
& +\partial_{\bar{z}} \sigma_{A}(z, \bar{z}) \partial_{\bar{z}} \sigma_{B}(z, \bar{z}) \partial_{\bar{w}} \sigma_{A}(w, \bar{w}) \partial_{\bar{w}} \sigma_{B}(w, \bar{w}) .
\end{aligned}
$$

Using the fact that only products of operators on the same sublattice yield a non-zero expansion and extracting the partial derivatives the last line is rewritten before applying the general normalized operator product expansion (given in (5.17)) for the spin-operator products:

$$
\begin{aligned}
= & \partial_{z} \partial_{w} \sigma_{A}(z, \bar{z}) \sigma_{A}(w, \bar{w}) \partial_{z} \partial_{w} \sigma_{B}(z, \bar{z}) \sigma_{B}(w, \bar{w}) \\
& -\partial_{z} \partial_{\bar{w}} \sigma_{A}(z, \bar{z}) \sigma_{A}(w, \bar{w}) \partial_{z} \partial_{\bar{w}} \sigma_{B}(z, \bar{z}) \sigma_{B}(w, \bar{w}) \\
& -\partial_{\bar{z}} \partial_{w} \sigma_{A}(z, \bar{z}) \sigma_{A}(w, \bar{w}) \partial_{\bar{z}} \partial_{w} \sigma_{B}(z, \bar{z}) \sigma_{B}(w, \bar{w}) \\
& +\partial_{\bar{z}} \partial_{\bar{w}} \sigma_{A}(z, \bar{z}) \sigma_{A}(w, \bar{w}) \partial_{\bar{z}} \partial_{\bar{w}} \sigma_{B}(z, \bar{z}) \sigma_{B}(w, \bar{w}) \\
= & \partial_{z} \partial_{w}\left(|z-w|^{-\frac{1}{4}}+\frac{1}{2}|z-w|^{\frac{3}{4}} \varepsilon_{A}(w, \bar{w})+\frac{1}{4}(z-w)^{\frac{11}{8}}(\bar{z}-\bar{w})^{\frac{3}{8}} \partial_{w} \varepsilon_{A}(w, \bar{w})+\text { H.c. }\right) \\
& \times \partial_{z} \partial_{w}\left(|z-w|^{-\frac{1}{4}}+\frac{1}{2}|z-w|^{\frac{3}{4}} \varepsilon_{B}(w, \bar{w})+\frac{1}{4}(z-w)^{\frac{11}{8}}(\bar{z}-\bar{w})^{\frac{3}{8}} \partial_{w} \varepsilon_{B}(w, \bar{w})+\text { H.c. }\right) \\
& -\partial_{z} \partial_{\bar{w}}(\ldots A) \partial_{z} \partial_{\bar{w}}\left(\ldots_{B}\right)-\partial_{\bar{z}} \partial_{w}\left(\ldots_{A}\right) \partial_{\bar{z}} \partial_{w}\left(\ldots_{B}\right)+\partial_{\bar{z}} \partial_{\bar{w}}\left(\ldots_{A}\right) \partial_{\bar{z}} \partial_{\bar{w}}\left(\cdots_{B}\right) .
\end{aligned}
$$


In the following only the regular terms and the pure energy terms are regarded for the sake of clarity. Note that $|z-w|^{x}=(z-w)^{\frac{x}{2}}(\bar{z}-\bar{w})^{\frac{x}{2}}$ enters into the calculation of the partial derivatives.

$$
\begin{aligned}
= & \left(-\frac{9}{64}(z-w)^{-\frac{17}{8}}(\bar{z}-\bar{w})^{-\frac{1}{8}}+\frac{15}{128}(z-w)^{-\frac{13}{8}}(\bar{z}-\bar{w})^{\frac{3}{8}} \varepsilon_{A}(w, \bar{w})+\mathcal{O}\left(\partial_{\{w, \bar{w}\}} \varepsilon_{A}\right)\right) \\
& \times\left(-\frac{9}{64}(z-w)^{-\frac{17}{8}}(\bar{z}-\bar{w})^{-\frac{1}{8}}+\frac{15}{128}(z-w)^{-\frac{13}{8}}(\bar{z}-\bar{w})^{\frac{3}{8}} \varepsilon_{B}(w, \bar{w})+\mathcal{O}\left(\partial_{\{w, \bar{w}\}} \varepsilon_{B}\right)\right) \\
& -2\left(-\frac{1}{64}(z-w)^{-\frac{9}{8}}(\bar{z}-\bar{w})^{-\frac{9}{8}}+\frac{9}{128}(z-w)^{-\frac{5}{8}}(\bar{z}-\bar{w})^{-\frac{5}{8}} \varepsilon_{A}(w, \bar{w})+\mathcal{O}\left(\partial_{\{w, \bar{w}\}} \varepsilon_{A}\right)\right) \\
& \times\left(-\frac{1}{64}(z-w)^{-\frac{9}{8}}(\bar{z}-\bar{w})^{-\frac{9}{8}}+\frac{9}{128}(z-w)^{-\frac{5}{8}}(\bar{z}-\bar{w})^{-\frac{5}{8}} \varepsilon_{B}(w, \bar{w})+\mathcal{O}\left(\partial_{\{w, \bar{w}\}} \varepsilon_{B}\right)\right) \\
& +\left(-\frac{9}{64}(z-w)^{-\frac{1}{8}}(\bar{z}-\bar{w})^{-\frac{17}{8}}+\frac{15}{128}(z-w)^{\frac{3}{8}}(\bar{z}-\bar{w})^{-\frac{13}{8}} \varepsilon_{A}(w, \bar{w})+\mathcal{O}\left(\partial_{\{w, \bar{w}\}} \varepsilon_{A}\right)\right) \\
& \times\left(-\frac{9}{64}(z-w)^{-\frac{1}{8}}(\bar{z}-\bar{w})^{-\frac{17}{8}}+\frac{15}{128}(z-w)^{\frac{3}{8}}(\bar{z}-\bar{w})^{-\frac{13}{8}} \varepsilon_{B}(w, \bar{w})+\mathcal{O}\left(\partial_{\{w, \bar{w}\}} \varepsilon_{B}\right)\right) .
\end{aligned}
$$

The calculation of the products $(\times)$ and summing over (B.11)-(B.13) yields

$$
\begin{aligned}
& {\left[\mathcal{O}_{1}(z, \bar{z})-\mathcal{O}_{2}(z, \bar{z})\right]\left[\mathcal{O}_{1}(w, \bar{w})-\mathcal{O}_{2}(w, \bar{w})\right] } \\
= & \frac{81}{4096}\left((z-w)^{-\frac{17}{4}}(\bar{z}-\bar{w})^{-\frac{1}{4}}+\text { H.c. }\right)-\frac{2}{4096}(z-w)^{-\frac{9}{4}}(\bar{z}-\bar{w})^{-\frac{9}{4}} \\
& -\left(\frac{135}{8192}\left((z-w)^{-\frac{15}{4}}(\bar{z}-\bar{w})^{\frac{1}{4}}+\text { H.c. }\right)-\frac{9}{4096}(z-w)^{-\frac{7}{4}}(\bar{z}-\bar{w})^{-\frac{7}{4}}\right)\left(\varepsilon_{A}+\varepsilon_{B}\right) \\
& +\left(\frac{225}{16384}\left((z-w)^{-\frac{13}{4}}(\bar{z}-\bar{w})^{\frac{3}{4}}+\text { H.c. }\right)-\frac{81}{8192}(z-w)^{-\frac{5}{4}}(\bar{z}-\bar{w})^{-\frac{5}{4}}\right) \varepsilon_{A} \varepsilon_{B} \\
& +\mathcal{O}\left(\partial_{\{w, \bar{w}\}} \varepsilon_{I}\right)
\end{aligned}
$$

which corresponds to the terms given in equations (5.20), (5.21) and (5.22).

\section{integration of prefactors $\mid$ B.2.2}

Before the computation of third order terms is performed, the structure of the prefactors $\propto(z-w)^{p} \cdot(\bar{z}-\bar{w})^{q}$ appearing in all second order terms is discussed. The integration of the prefactors is given in different orders by:

$$
\begin{aligned}
\text { first order: } & \iint d z d \bar{z} \\
\text { second order: } & \iint d z d \bar{z} \iint d w d \bar{w} \\
\text { third order: } & \iint d z d \bar{z} \iint d w d \bar{w} \iint d s d \bar{s} .
\end{aligned}
$$

Thus, the relation of the exponents $p$ and $q$ plays an important role. The only constraint for the integrals in second and third order is given by the lower cutoff $|z-w|>a$ and $|w-s|>a$, respectively. 
In second order the integration over $z$ and $\bar{z}$ can be carried out with respect to constant values of $w$ and $\bar{w}$ which leaves primary and secondary fields of $\varepsilon(w, \bar{w})$ untouched:

$$
\begin{aligned}
& \int_{|z-w|>a} d z d \bar{z} d w d \bar{w}(z-w)^{p}(\bar{z}-\bar{w})^{q} f(\varepsilon(w, \bar{w})) \\
& \stackrel{u=z-w}{=} \int_{|u|>a} d u d \bar{u} d w d \bar{w} u^{p} \bar{u}^{q} f(\varepsilon(w, \bar{w})) \\
& \stackrel{u=r e^{i \varphi}}{=} \int_{r>a} d r \int_{0}^{2 \pi} d \varphi \int d w d \bar{w} 2 r \cdot r^{p+q} e^{i \varphi(p-q)} f(\varepsilon(w, \bar{w})) .
\end{aligned}
$$

The transformation into polar coordinates $r$ and $\varphi$ in equation (B.17) and in particular the integration over the whole complex plane - except for a small circle around zero $r>a$ - leaves only terms which satisfy either $p=q$ or $p-q \neq n$ with $n \in \mathbb{Z}$. Thus, from the second order perturbation only the terms from equations (B.14), (B.15) and (B.16) are left that fulfill $p=q$, namely:

$$
-\frac{2}{4096}|z-w|^{-\frac{9}{2}}, \quad \frac{9}{4096}|z-w|^{-\frac{7}{2}}\left(\varepsilon_{A}+\varepsilon_{B}\right), \quad-\frac{81}{8192}|z-w|^{-\frac{5}{2}}\left(\varepsilon_{A} \varepsilon_{B}\right) .
$$

\section{third order calculations}

For the derivation of the third order perturbation all terms given in (B.18) are multiplied by the original operators from (5.13)

$$
\left[\mathcal{O}_{1}(s, \bar{s})-\mathcal{O}_{2}(s, \bar{s})\right]=\left[\partial_{s} \sigma_{A}(s, \bar{s}) \partial_{s} \sigma_{B}(s, \bar{s})-\partial_{\bar{s}} \sigma_{A}(s, \bar{s}) \partial_{\bar{s}} \sigma_{B}(s, \bar{s})\right]
$$

Since the regular term in (B.14) does not change the original spin operators it will be neglected here. For the operator product expansion of the bare energy terms in (B.15) one obtains (using (5.27) and naming $\sigma_{I}=\sigma_{I}(s, \bar{s})$ :

$$
\begin{aligned}
& \frac{9}{4096}|z-w|^{-\frac{7}{2}} \times \\
& {\left[\partial_{s}\left(\varepsilon_{A}(w, \bar{w}) \sigma_{A}(s, \bar{s})\right) \partial_{s} \sigma_{B}(s, \bar{s})-\partial_{\bar{s}}\left(\varepsilon_{A}(w, \bar{w}) \sigma_{A}(s, \bar{s})\right) \partial_{\bar{s}} \sigma_{B}(s, \bar{s})\right.} \\
& \left.+\partial_{s} \sigma_{A}(s, \bar{s}) \partial_{s}\left(\varepsilon_{B}(w, \bar{w}) \sigma_{B}(s, \bar{s})\right)-\partial_{\bar{s}} \sigma_{A}(s, \bar{s}) \partial_{\bar{s}}\left(\varepsilon_{B}(w, \bar{w}) \sigma_{B}(s, \bar{s})\right)\right] \\
= & \frac{9}{4096}|z-w|^{-\frac{7}{2}} \times \\
& \left\{\left[\frac{1}{4}(w-s)^{-\frac{3}{2}}(\bar{w}-\bar{s})^{-\frac{1}{2}} \sigma_{A} \partial_{s} \sigma_{B}+\frac{1}{2}|w-s|^{-1} \partial_{s} \sigma_{A} \partial_{s} \sigma_{B}+\frac{1}{8}|w-s|^{-1} \partial_{s} \sigma_{A} \partial_{s} \sigma_{B}\right.\right. \\
& \left.-\frac{1}{8}(w-s)^{-\frac{3}{2}}(\bar{w}-\bar{s})^{\frac{1}{2}} \partial_{\bar{s}} \sigma_{A} \partial_{s} \sigma_{B}+\mathcal{O}\left(\partial_{\{s, \bar{s}\}}^{2} \sigma_{A}\right) \partial_{s} \sigma_{B}-\text { H.c. }\right] \\
& +\left[\frac{1}{4}(w-s)^{-\frac{3}{2}}(\bar{w}-\bar{s})^{-\frac{1}{2}} \partial_{s} \sigma_{A} \sigma_{B}+\frac{1}{2}|w-s|^{-1} \partial_{s} \sigma_{A} \partial_{s} \sigma_{B}+\frac{1}{8}|w-s|^{-1} \partial_{s} \sigma_{A} \partial_{s} \sigma_{B}\right. \\
& \left.\left.-\frac{1}{8}(w-s)^{-\frac{3}{2}}(\bar{w}-\bar{s})^{\frac{1}{2}} \partial_{s} \sigma_{A} \partial_{\bar{s}} \sigma_{B}+\partial_{s} \sigma_{A} \mathcal{O}\left(\partial_{\{s, \bar{s}\}}^{2} \sigma_{B}\right)-\text { H.c. }\right]\right\} .
\end{aligned}
$$


The only terms with prefactors that will not be canceled by integrating over $d w d \bar{w}$ have the exact same structure as the first order terms. The Ashkin-Teller term from equation (B.16) yields

$$
\begin{aligned}
& -\frac{81}{8192}|z-w|^{-\frac{5}{2}} \times \\
& {\left[\partial_{s}\left(\varepsilon_{A}(w, \bar{w}) \sigma_{A}(s, \bar{s})\right) \partial_{s}\left(\varepsilon_{B}(w, \bar{w}) \sigma_{B}(s, \bar{s})\right)-\partial_{\bar{s}}\left(\varepsilon_{A}(w, \bar{w}) \sigma_{A}(s, \bar{s})\right) \partial_{\bar{s}}\left(\varepsilon_{B}(w, \bar{w}) \sigma_{B}(s, \bar{s})\right)\right] } \\
= & -\frac{81}{8192}|z-w|^{-\frac{5}{2}} \times \\
& \left\{\left[\frac{1}{4}(w-s)^{-\frac{3}{2}}(\bar{w}-\bar{w})^{-\frac{1}{2}} \sigma_{A}+\frac{3}{8}|w-s|^{-1} \partial_{s} \sigma_{A}-\frac{1}{8}(w-s)^{-\frac{3}{2}}(\bar{w}-\bar{s})^{\frac{1}{2}} \partial_{\bar{s}} \sigma_{A}\right] \cdot[\cdots B]-\text { H.c. }\right\} \\
= & -\frac{81}{8192}|z-w|^{-\frac{5}{2}} \times \\
& \left\{\left[\frac{1}{16}(w-s)^{-3}(\bar{w}-\bar{s})^{-1} \sigma_{A} \sigma_{B}+\frac{3}{32}(w-s)^{-2}(\bar{w}-\bar{s})^{-1}\left(\sigma_{A} \partial_{s} \sigma_{B}+\partial_{s} \sigma_{A} \sigma_{B}\right)\right.\right. \\
& \quad-\frac{1}{32}(w-s)^{-3}\left(\sigma_{A} \partial_{\bar{s}} \sigma_{B}+\partial_{\bar{s}} \sigma_{A} \sigma_{B}\right)+\frac{9}{64}|w-s|^{-2} \partial_{s} \sigma_{A} \partial_{s} \sigma_{B} \\
& \left.\left.\quad-\frac{3}{64}(w-s)^{-2}\left(\partial_{s} \sigma_{A} \partial_{\bar{s}} \sigma_{B}+\partial_{\bar{s}} \sigma_{A} \partial_{s} \sigma_{B}\right)\right]- \text { H.c. }\right\}
\end{aligned}
$$

which again leaves only the irrelevant first order terms after the integration. 
list of references 



\section{in alphabetical order of labels}

[AAC ${ }^{+}$07] Albuquerque A., Alet F., Corboz P., Dayal P., Feiguin A., Fuchs S., Gamper L., Gull E., Guertler S., Honecker A., Igarashi R., Koerner M., Kozhevnikov A., Laeuchli A., Manmana S., Matsumoto M., McCulloch I., Michel F., Noack R., Pawłowski G. and Wessel S., The ALPS project release 1.3: Open-source software for strongly correlated systems. Journal of Magnetism and Magnetic Materials, 310, 1187 (2007), URL http://dx.doi.org/10. 1016/j.jmmm. 2006 .10.304.

[AAL01] Allen D., Azaria P. and Lecheminant P., A two-leg quantum Ising ladder: a bosonization study of the ANNNI model. Journal of Physics A: Mathematical and General, 34, L305 (2001), URL http://dx.doi.org/10.1088/0305-4470/34/21/101.

[ADG $\left.{ }^{+} 05\right]$ Alet F., Dayal P., Grzesik A., Honecker A., Körner M., Läuchli A., Manmana S.R., McCulloch I.P., Michel F., Noack R.M., Schmid G., Schollwöck U., Stöckli F., Todo S., Trebst S., Troyer M., Werner P. and Wessel S., The ALPS Project: Open Source Software for Strongly Correlated Systems. Journal of the Physical Society of Japan, 74, S30 (2005), URL http:// $\mathrm{dx}$.doi.org/10.1143/JPS JS.74S.30.

[Aff86] Affleck I., Universal term in the free energy at a critical point and the conformal anomaly. Physical Review Letters, 56, 746 (1986), URL http://dx.doi.org/10.1103/ PhysRevLett.56.746.

[And73] Anderson P.W., Resonating valence bonds: A new kind of insulator? Materials Research Bulletin, 8, 153 (1973), URL http://dx.doi.org/10.1016/0025-5408(73)90167-0.

[And87] Anderson P.W., The Resonating Valence Bond State in $\mathrm{La}_{2} \mathrm{CuO}_{4}$ and Superconductivity. Science, 235, 1196 (1987), URL http://dx.doi.org/10.1126/science.235.4793.1196.

[And92] Anderson P.W., Experimental constraints on the theory of high-T $T_{C}$ superconductivity. Science, 256, 1526 (1992), URL http://dx.doi.org/10.1126/science.256.5063.1526.

[And97] Anderson P.W., Theory of Superconductivity in the High-T $T_{C}$ Cuprates, (Princeton University Press, Princeton, 1997), 1st edition.

[ASH $\left.{ }^{+} 11\right]$ Albuquerque A., Schwandt D., Hetényi B., Capponi S., Mambrini M. and Läuchli A., Phase diagram of a frustrated quantum antiferromagnet on the honeycomb lattice: Magnetic order versus valence-bond crystal formation. Physical Review B, 84, 024406 (2011), URL http: // dx.doi.org/10.1103/PhysRevB.84.024406.

[AT43] Ashkin J. and Teller E., Statistics of Two-Dimensional Lattices with Four Components. Physical Review, 64, 178 (1943), URL http://dx.doi.org/10.1103/PhysRev.64.178.

[AWT05] Alet F., Wessel S. and Troyer M., Generalized directed loop method for quantum Monte Carlo simulations. Physical Review E, 71, 036706 (2005), URL http://dx.doi.org/10. 1103/PhysRevE.71.036706.

[Bak82] Bak P., Commensurate phases, incommensurate phases and the devil's staircase. Reports on Progress in Physics, 45, 587 (1982), URL http://dx.doi.org/10.1088/0034-4885/ 45/6/001.

[Bal10] Balents L., Spin liquids in frustrated magnets. Nature, 464, 199 (2010), URL http://dx. doi.org/10.1038/nature08917.

[Bax82] Baxter R.J., Exactly solved models in statistical mechanics, (Academic Press, London, 1982), URL http://tpsrv. anu.edu.au/research/mathphys/Book111.pdf.

$\left[\mathrm{BBB}^{+} 05\right] \quad$ Balents L., Bartosch L., Burkov A., Sachdev S. and Sengupta K., Putting competing orders in their place near the Mott transition. Physical Review B, 71, 144508 (2005), URL http:// $\mathrm{dx}$.doi.org/10.1103/PhysRevB.71.144508. 
[BBT02] Bernardet K., Batrouni G.G. and Troyer M., Destruction of diagonal and off-diagonal longrange order by disorder in two-dimensional hard-core boson systems. Physical Review B, 66, 054520 (2002), URL http://dx.doi.org/10.1103/PhysRevB.66.054520.

$\left[\mathrm{BCE}^{+} 11\right]$ Bauer B., Carr L.D., Evertz H.G., Feiguin A., Freire J., Fuchs S., Gamper L., Gukelberger J., Gull E., Guertler S., Hehn A., Igarashi R., Isakov S.V., Koop D., Ma P.N., Mates P., Matsuo H., Parcollet O., Pawłowski G., Picon J.D., Pollet L., Santos E., Scarola V.W., Schollwöck U., Silva C., Surer B., Todo S., Trebst S., Troyer M., Wall M.L., Werner P. and Wessel S., The ALPS project release 2.0: open source software for strongly correlated systems. Journal of Statistical Mechanics: Theory and Experiment, page P05001 (2011), URL http://dx.doi.org/10.1088/1742-5468/2011/05/P05001.

[BCN86] Blöte H.W.J., Cardy J.L. and Nightingale M.P., Conformal invariance, the central charge, and universal finite-size amplitudes at criticality. Physical Review Letters, 56, 742 (1986), URL http://dx.doi.org/10.1103/PhysRevLett.56.742.

[Ber04] Berg B.A., Markov Chain Monte Carlo Simulations and Their Statistical Analysis, (World Scientific, Singapore, 2004), 1st edition.

[Bet31] Bethe H., Zur Theorie der Metalle. Zeitschrift für Physik, 71, 205 (1931), URL http:// dx.doi.org/10.1007/BF01341708.

[BF64] Bonner J.C. and Fisher M.E., Linear Magnetic Chains with Anisotropic Coupling. Physical Review, 135, A640 (1964), URL http://dx.doi.org/10.1103/PhysRev.135.A640.

[Bin81a] Binder K., Critical Properties from Monte Carlo Coarse Graining and Renormalization. Physical Review Letters, 47, 693 (1981), URL http://dx.doi.org/10.1103/ PhysRevLett .47.693.

[Bin81b] Binder K., Finite size scaling analysis of Ising model block distribution functions. Zeitschrift für Physik B Condensed Matter, 43, 119 (1981), URL http://dx.doi.org/10.1007/ BF01293604.

[BL80] Binder K. and Landau D.P., Phase diagrams and critical behavior in Ising square lattices with nearest- and next-nearest-neighbor interactions. Physical Review B, 21, 1941 (1980), URL http://dx.doi.org/10.1103/PhysRevB.21.1941.

[BLDR08] Bishop R.F., Li P.H.Y., Darradi R. and Richter J., The quantum $J_{1}-J_{1}{ }^{\prime}-J_{2}$ spin-1/2 Heisenberg model: influence of the interchain coupling on the ground-state magnetic ordering in two dimensions. Journal of Physics: Condensed Matter, 20, 255251 (2008), URL http:// dx.doi.org/10.1088/0953-8984/20/25/255251.

[Blo32] Bloch F., Zur Theorie des Austauschproblems und der Remanenzerscheinung der Ferromagnetika. Zeitschrift für Physik, 74, 295 (1932), URL http://dx.doi.org/10.1007/ BF01337791.

[BM86] Bednorz J.G. and Müller K.A., Possible high $T_{C}$ superconductivity in the Ba-La-Cu-O system. Zeitschrift für Physik B Condensed Matter, 64, 189 (1986), URL http://dx.doi. org/10.1007/BF01303701.

[BPZ84a] Belavin A.A., Polyakov A.M. and Zamolodchikov A.B., Infinite conformal symmetry in two-dimensional quantum field theory. Nuclear Physics B, 241, 333 (1984), URL http:// dx.doi.org/10.1016/0550-3213(84)90052-X.

[BPZ84b] Belavin A.A., Polyakov A.M. and Zamolodchikov A.B., Infinite conformal symmetry of critical fluctuations in two dimensions. Journal of Statistical Physics, 34, 763 (1984), URL http://dx.doi.org/10.1007/BF01009438.

[BS00] Batrouni G.G. and Scalettar R.T., Phase Separation in Supersolids. Physical Review Letters, 84, 1599 (2000), URL http://dx.doi.org/10.1103/PhysRevLett.84.1599.

[BSFB07] Bergman D., Shindou R., Fiete G. and Balents L., Degenerate perturbation theory of quantum fluctuations in a pyrochlore antiferromagnet. Physical Review B, 75, 094403 (2007), URL http://dx.doi.org/10.1103/PhysRevB.75.094403.

[BvB80] Bak P. and von Boehm J., Ising model with solitons, phasons, and "the devil's staircase". Physical Review B, 21, 5297 (1980), URL http://dx.doi.org/10.1103/PhysRevB.21. 5297. 
[Car96] Cardy J., Scaling and Renormalization in Statistical Physics, in Cambridge Lecture Notes in Physics (edited by P. Goddard and J. Yeomans), volume 5, (Campridge University Press, Cambridge, 1996).

[CAS11] Clark B.K., Abanin D.A. and Sondhi S.L., Nature of the Spin Liquid State of the Hubbard Model on a Honeycomb Lattice. Physical Review Letters, 107, 087204 (2011), URL http:// dx.doi.org/10.1103/PhysRevLett.107.087204.

[CBPS03] Capriotti L., Becca F., Parola A. and Sorella S., Suppression of dimer correlations in the two-dimensional $J_{1}-J_{2}$ Heisenberg model: An exact diagonalization study. Physical Review B, 67, 212402 (2003), URL http://dx.doi.org/10.1103/PhysRevB.67.212402.

$\left[\mathrm{CDK}^{+} 00\right]$ Chandra V.R., Dagum L., Kohr D., Menon R., Maydan D. and McDonald J., Parallel programming in OpenMP, (Morgan Kaufmann, 2000), 1st edition.

[CEE08] Chubukov A.V., Efremov D.V. and Eremin I., Magnetism, superconductivity, and pairing symmetry in iron-based superconductors. Physical Review B, 78, 134512 (2008), URL http://dx.doi.org/10.1103/PhysRevB.78.134512.

[CG05] Chitov G.Y. and Gros C., Ordering in two-dimensional Ising models with competing interactions. Low Temperature Physics, 31, 722 (2005), URL http://dx.doi.org/10.1063/1. 2008132.

$\left[\mathrm{CGP}^{+}\right.$09] Castro Neto A.H., Guinea F., Peres N.M.R., Novoselov K.S. and Geim A.K., The electronic properties of graphene. Reviews of Modern Physics, 81, 109 (2009), URL http://dx.doi. org/10.1103/RevModPhys.81.109.

[CJvdP07] Chapman B., Jost G. and van der Pas R., Using OpenMP: portable shared memory parallel programming, (MIT Press, 2007), 1st edition.

[CL98] Canals B. and Lacroix C., Pyrochlore Antiferromagnet: A Three-Dimensional Quantum Spin Liquid. Physical Review Letters, 80, 2933 (1998), URL http://dx . doi .org/10.1103/ PhysRevLett.80.2933.

[CLR11] Cabra D., Lamas C. and Rosales H., Quantum disordered phase on the frustrated honeycomb lattice. Physical Review B, 83, 094506 (2011), URL http://dx.doi.org/10.1103/ PhysRevB.83.094506.

[CMWK08] Chen Y.C., Melko R., Wessel S. and Kao Y.J., Supersolidity from defect condensation in the extended boson Hubbard model. Physical Review B, 77, 014524 (2008), URL http:// dx.doi.org/10.1103/PhysRevB.77.014524.

[CS09] Chelkak D. and Smirnov S., Universality in the 2D Ising model and conformal invariance of fermionic observables. preprint: 0910.2045 (2009), URL http://arxiv.org/abs/0910. 2045.

[CTTT01] Coldea R., Tennant D.A., Tsvelik A.M. and Tylczynski Z., Experimental Realization of a 2D Fractional Quantum Spin Liquid. Physical Review Letters, 86, 1335 (2001), URL http:// dx.doi.org/10.1103/PhysRevLett.86.1335.

[dFMS97] di Francesco P., Mathieu P. and Sénéchal D., Conformal Field Theory, (Springer, New York, 1997), 1st edition.

[DG04] Delfino G. and Grinza P., Universal ratios along a line of critical points. The Ashkin-Teller model. Nuclear Physics B, 682, 521 (2004), URL http://dx.doi.org/10.1016/j. nuclphysb.2004.01.007.

[Die05] Diep H.T. (editor), Frustrated Spin Systems, (World Scientific, Singapore, 2005), 1st edition.

[DM98] Delfino G. and Mussardo G., Non-integrable aspects of the multi-frequency sineGordon model. Nuclear Physics B, 516, 675 (1998), URL http://dx.doi.org/10.1016/ S0550-3213 (98) 00063-7.

[dNNS82] den Nijs M., Nightingale M. and Schick M., Critical fan in the antiferromagnetic three-state Potts model. Physical Review B, 26, 2490 (1982), URL http://dx.doi.org/10.1103/ PhysRevB.26.2490.

[EKL90] Emery V., Kivelson S. and Lin H., Phase separation in the t-J model. Physical Review Letters, 64, 475 (1990), URL http://dx.doi.org/10.1103/PhysRevLett.64.475. 
[FB04] Farnell D.J. and Bishop R.F., The Coupled Cluster Method Applied to Quantum Magnetism, in Quantum Magnetism (edited by U. Schollwöck, J. Richter, D.J.J. Farnell and R.F. Bishop), volume 645 of Lecture Notes in Physics, pages 307-348, (Springer, Berlin, 2004), URL http://www. springerlink. com/content/xdwj3hv2nkdfqn3p/.

$\left[\mathrm{FML}^{+} 78\right]$ Fischer P., Meier G., Lebech B., Rainford B.D. and Vogt O., Magnetic phase transitions of CeSb. I. Zero applied magnetic field. Journal of Physics C: Solid State Physics, 11, 345 (1978), URL http://dx.doi.org/10.1088/0022-3719/11/2/018.

[FQS84] Friedan D., Qiu Z. and Shenker S., Conformal Invariance, Unitarity, and Critical Exponents in Two Dimensions. Physical Review Letters, 52, 1575 (1984), URL http://dx.doi.org/ 10.1103/PhysRevLett. 52.1575.

[FSL01] Fouet J., Sindzingre P. and Lhuillier C., An investigation of the quantum $J_{1}-J_{2}-J_{3}$ model on the honeycomb lattice. The European Physical Journal B, 20, 241 (2001), URL http:// dx.doi.org/10.1007/s100510170273.

[FW70] Fan C. and Wu F., General Lattice Model of Phase Transitions. Physical Review B, 2, 723 (1970), URL http://dx.doi.org/10.1103/PhysRevB.2.723.

[GFB $\left.{ }^{+} 04\right]$ Gabriel E., Fagg G.E., Bosilca G., Angskun T., Dongarra J.J., Squyres J.M., Sahay V., Kambadur P., Barrett B., Lumsdaine A., Castain R.H., Daniel D.J., Graham R.L. and Woodall T.S., Open MPI: Goals, Concept, and Design of a Next Generation MPI Implementation, in Proceedings, 11th European PVM/MPI Users' Group Meeting, pages 97-104, (Budapest, Hungary, 2004).

[GG74] Gaunt D.S. and Guttmann A.J., Asymptotic Analysis of Coefficients, in Phase Transitions and Critical Phenomena (edited by C. Domb and M.S. Greene), (Academic Press, London, 1974), 1st edition.

[GGLM08] Ghoufi A., Goujon F., Lachet V. and Malfreyt P., Multiple histogram reweighting method for the surface tension calculation. The Journal of Chemical Pghysics, 128, 154718 (2008), URL http://dx.doi.org/10.1063/1.2904460.

[Gin88] Ginsparg P., Applied Conformal Field Theory, in Fields, strings and critical phenomena (edited by E. Brézin and J. Zinn Justin), (Session XLIX, Les Houches, 1988), URL http:// arxiv.org/abs/hep-th/9108028.

[GNT04] Gogolin A.O., Nersesyan A.A. and Tsvelik A.M., Bosonization and strongly correlated systems, (Cambridge University Press, Cambridge, 2004), 1st edition.

[Gol93] Goldenfeld N., Lectures on phase transitions and the renormalization group, (AddisonWesley Publishing Company, 1993), 3rd edition.

[GvL96] Golub G.H. and van Loan C.F., Matrix Computations, (The John Hopkins University Press, Baltimore, 1996), 3rd edition.

[Han62] Handscomb D.C., The Monte Carlo method in quantum statistical mechanics. Mathematical Proceedings of the Cambridge Philosophical Society, 58, 594 (1962), URL http://dx.doi. org/10.1017/S0305004100040639.

[Han97] Hansmann U.H., Parallel tempering algorithm for conformational studies of biological molecules. Chemical Physics Letters, 281, 140 (1997), URL http://dx.doi.org/10.1016/ S0009-2614 (97) 01198-6.

[HBS ${ }^{+}$01] Hébert F., Batrouni G.G., Schmid G., Troyer M. and Dorneich A., Quantum phase transitions in the two-dimensional hardcore boson model. Physical Review B, 65, 014513 (2001), URL http://dx.doi.org/10.1103/PhysRevB.65.014513.

[HD05] Heidarian D. and Damle K., Persistent Supersolid Phase of Hard-Core Bosons on the Triangular Lattice. Physical Review Letters, 95, 127206 (2005), URL http://dx.doi.org/ 10.1103/PhysRevLett.95.127206.

[Hei28] Heisenberg W., Zur Theorie des Ferromagnetismus. Zeitschrift für Physik, 49, 619 (1928), URL http://dx.doi.org/10.1007/BF01328601.

[HN96] Hukushima K. and Nemoto K., Exchange Monte Carlo Method and Application to Spin Glass Simulations. Journal of the Physical Society of Japan, 65, 1604 (1996), URL http: // dx.doi.org/10.1143/JPS J.65.1604. 
[Hoe50] Hoehler G., Ferromagnetismus als Einstein-Kondensation der Blochschen Spinwellen. Annalen der Physik, 442, 93 (1950), URL http://dx.doi.org/10.1002/andp.19504420110.

[Hoh67] Hohenberg P., Existence of Long-Range Order in One and Two Dimensions. Physical Review, 158, 383 (1967), URL http://dx.doi.org/10.1103/PhysRev.158.383.

[HP40] Holstein T. and Primakoff H., Field Dependence of the Intrinsic Domain Magnetization of a Ferromagnet. Physical Review, 58, 1098 (1940), URL http://dx.doi.org/10.1103/ PhysRev.58.1098.

[IS04] Ivanov N.B. and Sen D., Spin Wave Analysis of Heisenberg Magnets in Restricted Geometries, in Quantum Magnetism (edited by U. Schollwöck, J. Richter, D.J.J. Farnell and R.F. Bishop), volume 645 of Lecture Notes in Physics, pages 195-226, (Springer, Berlin, 2004), URL http: //www.springerlink.com/content/032cp1gjwghdurd5/.

[Isi25] Ising E., Beitrag zur Theorie des Ferromagnetismus. Zeitschrift für Physik, 31, 253 (1925), URL http://dx.doi.org/10.1007/BF02980577.

[JGT $\left.{ }^{+} 12\right] \quad$ Jo G.B., Guzman J., Thomas C., Hosur P., Vishwanath A. and Stamper-Kurn D., Ultracold Atoms in a Tunable Optical Kagome Lattice. Physical Review Letters, 108, 045305 (2012), URL http://dx.doi.org/10.1103/PhysRevLett.108.045305.

[Jia11] Jiang F.J., Method of calculating the spin-wave velocity of spin-1/2 antiferromagnets with $O(N)$ symmetry in a Monte Carlo simulation. Physical Review B, 83, 024419 (2011), URL http://dx.doi.org/10.1103/PhysRevB.83.024419.

[Joy67] Joyce G., Classical Heisenberg Model. Physical Review, 155, 478 (1967), URL http://dx . doi.org/10.1103/PhysRev.155.478.

[JSS12] Jin S., Sen A. and Sandvik A., Ashkin-Teller Criticality and Pseudo-First-Order Behavior in a Frustrated Ising Model on the Square Lattice. Physical Review Letters, 108, 045702 (2012), URL http://dx.doi.org/10.1103/PhysRevLett.108.045702.

[JYB11] Jiang H.C., Yao H. and Balents L., Spin Liquid Ground State of the Spin-1/2 Square $J_{1}-J_{2}$ Heisenberg Model. preprint: 1112.2241 (2011), URL http://arxiv.org/abs/1112.2241.

$\left[\mathrm{KAC}^{+}{ }^{12}\right] \quad$ Kalz A., Arlego M., Cabra D., Honecker A. and Rossini G., Anisotropic frustrated Heisenberg model on the honeycomb lattice. Physical Review B, 85, 104505 (2012), URL http://dx. doi.org/10.1103/PhysRevB.85.104505.

[Kal08] Kalz A., Monte-Carlo Simulationen für frustrierte zweidimendionale Gittermodelle, Diplomarbeit, Georg-August Universität Göttingen (2008), URL http://www.theorie.physik. uni-goettingen. de $/ \% 7$ ekalz.

[KHFP08] Kalz A., Honecker A., Fuchs S. and Pruschke T., Phase diagram of the Ising square lattice with competing interactions. The European Physical Journal B, 65, 533 (2008), URL http://dx.doi.org/10.1140/epjb/e2008-00359-6.

[KHFP09] Kalz A., Honecker A., Fuchs S. and Pruschke T., Monte Carlo studies of the Ising square lattice with competing interactions. Journal of Physics: Conference Series, 145, 012051 (2009), URL http://dx.doi.org/10.1088/1742-6596/145/1/012051.

[KHFP11a] Kalz A., Honecker A., Fuchs S. and Pruschke T., Quantum disordered ground state for hardcore bosons on the frustrated square lattice (+ Erratum Phys. Rev. B 84, 219902(E) (2011)). Physical Review B, 83, 174519 (2011), URL http://dx.doi.org/10.1103/PhysRevB.83. 174519.

[KHFP11b] Kalz A., Honecker A., Fuchs S. and Pruschke T., Quantum disordered ground state for the frustrated square lattice. preprint: 1110.3162 (2011), URL http://arxiv.org/abs/1110. 3162.

[KHM11] Kalz A., Honecker A. and Moliner M., Analysis of the phase transition for the Ising model on the frustrated square lattice. Physical Review B, 84, 174407 (2011), URL http://dx. doi.org/10.1103/PhysRevB.84.174407.

[Kit06] Kitaev A., Anyons in an exactly solved model and beyond. Annals of Physics, 321, 2 (2006), URL http://dx.doi.org/10.1016/j.aop.2005.10.005. 
[KL87] Kalmeyer V. and Laughlin R., Equivalence of the resonating-valence-bond and fractional quantum Hall states. Physical Review Letters, 59, 2095 (1987), URL http://dx.doi.org/ 10.1103/PhysRevLett.59.2095.

[Kog79] Kogut J.B., An introduction to lattice gauge theory and spin systems. Reviews of Modern Physics, 51, 659 (1979), URL http://dx.doi.org/10.1103/RevModPhys.51.659.

[KT73] Kosterlitz J.M. and Thouless D.J., Ordering, metastability and phase transitions in twodimensional systems. Journal of Physics C: Solid State Physics, 6, 1181 (1973), URL http://dx.doi.org/10.1088/0022-3719/6/7/010.

[KTHT06] Katzgraber H.G., Trebst S., Huse D.A. and Troyer M., Feedback-optimized parallel tempering Monte Carlo. Journal of Statistical Mechanics: Theory and Experiment, page P03018 (2006), URL http://dx.doi.org/10.1088/1742-5468/2006/03/P03018.

[KW71] Kadanoff L. and Wegner F., Some Critical Properties of the Eight-Vertex Model. Physical Review B, 4, 3989 (1971), URL http://dx.doi.org/10.1103/PhysRevB.4.3989.

[KY11] Kimchi I. and You Y.Z., Kitaev-Heisenberg- $J_{2}-J_{3}$ model for the iridates $A_{2} \mathrm{IrO}_{3}$. Physical Review B, 84, 180407(R) (2011), URL http://dx.doi.org/10.1103/PhysRevB.84. 180407.

[Lan80] Landau D., Phase transitions in the Ising square lattice with next-nearest-neighbor interactions. Physical Review B, 21, 1285 (1980), URL http://dx.doi.org/10.1103/PhysRevB. 21.1285.

[LB85] Landau D. and Binder K., Phase diagrams and critical behavior of Ising square lattices with nearest-, next-nearest-, and third-nearest-neighbor couplings. Physical Review B, 31, 5946 (1985), URL http://dx.doi.org/10.1103/PhysRevB.31.5946.

[LB05] Landau D.P. and Binder K., Monte Carlo Simulations in Statistical Physics, (Cambridge University Press, Cambridge, 2005), 2nd edition.

[Len20] Lenz W., Beitrag zum Verständnis der magnetischen Erscheinungen in festen Körpern. Physikalische Zeitschrift, 21, 613 (1920).

[LF73] Liu K.S. and Fisher M.E., Quantum lattice gas and the existence of a supersolid. Journal of Low Temperature Physics, 10, 655 (1973), URL http://dx.doi.org/10.1007/ BF00655458.

[LMM11] Lacroix C., Mendels P. and Mila F. (editors), Introduction to Frustrated Magnetism, volume 164 of Springer Series in Solid-State Sciences, (Springer, Berlin, 2011), 1st edition, URL http://www. springer . com/materials/book/978-3-642-10588-3.

[LMSS12] Laumann C., Moessner R., Scardicchio A. and Sondhi S., Statistical Mechanics of Classical and Quantum Computational Complexity, in Modern Theories of Many-Particle Systems in Condensed Matter Physics (edited by D.C. Cabra, A. Honecker and P. Pujol), volume 843 of Lecture Notes in Physics, pages 295-332, (Springer, Berlin, 2012), URL http://dx. doi. org/10.1007/978-3-642-10449-7.

[LNW06] Lee P.A., Nagaosa N. and Wen X.G., Doping a Mott insulator: Physics of high-temperature superconductivity. Reviews of Modern Physics, 78, 17 (2006), URL http://dx.doi.org/ 10.1103/RevModPhys.78.17.

[LP04] Laflorencie N. and Poilblanc D., Simulations of Pure and Doped Low-Dimensional Spin$1 / 2$ Gapped Systems, in Quantum Magnetism (edited by U. Schollwöck, J. Richter, D.J.J. Farnell and R.F. Bishop), volume 645 of Lecture Notes in Physics, pages 227-252, (Springer, Berlin, 2004), URL http://www. springerlink. com/content/wy5qy2kqk9v6yvj1/.

$\left[\mathrm{LSA}^{+}\right.$07] Lewenstein M., Sanpera A., Ahufinger V., Damski B., Sen(De) A. and Sen U., Ultracold atomic gases in optical lattices: mimicking condensed matter physics and beyond. Advances in Physics, 56, 243 (2007), URL http://dx.doi.org/10.1080/00018730701223200.

$\left[\mathrm{LWB}^{+} 00\right] \quad$ Lefebvre S., Wzietek P., Brown S., Bourbonnais C., Jérome D., Mézière C., Fourmigué M. and Batail P., Mott Transition, Antiferromagnetism, and Unconventional Superconductivity in Layered Organic Superconductors. Physical Review Letters, 85, 5420 (2000), URL http://dx.doi.org/10.1103/PhysRevLett.85.5420. 
[Mah00] Mahan G., Many particle physics, (Springer, New York, 2000), 3rd edition.

[Maj10] Majumdar K., Second-order quantum corrections for the frustrated spatially anisotropic spin-1/2 Heisenberg antiferromagnet on a square lattice. Physical Review B, 82, 144407 (2010), URL http://dx.doi.org/10.1103/PhysRevB.82.144407.

[MB12] Mezzacapo F. and Boninsegni M., Ground-state phase diagram of the quantum $J_{1}-J_{2}$ model on the honeycomb lattice. Physical Review B, 85, 060402(R) (2012), URL http://dx.doi. org/10.1103/PhysRevB.85.060402.

[McK97] McKenzie R.H., Similarities Between Organic and Cuprate Superconductors. Science, 278, 820 (1997), URL http://dx.doi.org/10.1126/science.278.5339.820.

[MCPR95] Mascagni M., Cuccaro S.A., Pryor D.V. and Robinson M.L., A fast, high quality, and reproducible parallel lagged-Fibonacci pseudorandom number generator. Journal of Computational Physics, 119, 211 (1995), URL http://dx.doi.org/10.1006/jcph.1995.1130.

[Mel07] Melko R.G., Simulations of quantum XXZ models on two-dimensional frustrated lattices. Journal of Physics: Condensed Matter, 19, 145203 (2007), URL http://dx.doi.org/10. 1088/0953-8984/19/14/145203.

[MGCP10] Mulder A., Ganesh R., Capriotti L. and Paramekanti A., Spiral order by disorder and lattice nematic order in a frustrated Heisenberg antiferromagnet on the honeycomb lattice. Physical Review B, 81, 214419 (2010), URL http://dx.doi.org/10.1103/PhysRevB.81.214419.

[MI11] Murtazaev A.K. and Ibaev Z.G., Critical properties of the anisotropic Ising model with competing interactions. Journal of Experimental and Theoretical Physics, 113, 106 (2011), URL http://dx.doi.org/10.1134/S1063776111050165.

[MKT06] Malakis A., Kalozoumis P. and Tyraskis N., Monte Carlo studies of the square Ising model with next-nearest-neighbor interactions. The European Physical Journal B, 50, 63 (2006), URL http://dx.doi.org/10.1140/epjb/e2006-00032-2.

[ML05] Misguich G. and Lhuillier C., Two-dimensional Quantum Antiferromagnets, in Frustrated spin systems (edited by H.T. Diep), chapter 5., (World Scientific, Singapore, 2005), 1st edition, URL http://arxiv.org/abs/cond-mat/0310405.

[MLAGS93] Morán-López J., Aguilera-Granja F. and Sanchez J., First-order phase transitions in the Ising square lattice with first- and second-neighbor interactions. Physical Review B, 48, 3519 (1993), URL http://dx.doi.org/10.1103/PhysRevB.48.3519.

[MLAGS94] Morán-López J., Aguilera-Granja F. and Sanchez J., Phase transitions in Ising square antiferromagnets with first- and second-neighbour interactions. Journal of Physics: Condensed Matter, 6, 9759 (1994), URL http://dx.doi.org/10.1088/0953-8984/6/45/025.

[MLMS02] Misguich G., Lhuillier C., Mambrini M. and Sindzingre P., Degeneracy of the ground-state of antiferromagnetic spin-1/2 Hamiltonians. The European Physical Journal B, 26, 167 (2002), URL http://dx.doi.org/10.1140/epjb/e20020078.

[MLPM06] Mambrini M., Läuchli A., Poilblanc D. and Mila F., Plaquette valence-bond crystal in the frustrated Heisenberg quantum antiferromagnet on the square lattice. Physical Review B, 74, 144422 (2006), URL http://dx.doi.org/10.1103/PhysRevB.74.144422.

$\left[\mathrm{MLW}^{+}\right.$10] Meng Z.Y., Lang T.C., Wessel S., Assaad F.F. and Muramatsu A., Quantum spin liquid emerging in two-dimensional correlated Dirac fermions. Nature, 464, 847 (2010), URL http://dx.doi.org/10.1038/nature08942.

[MM56] Matsubara T. and Matsuda H., A Lattice Model of Liquid Helium, I. Progress of Theoretical Physics, 16, 569 (1956), URL http://dx.doi.org/10.1143/PTP.16.569.

[MN98] Matsumoto M. and Nishimura T., Mersenne twister: a 623-dimensionally equidistributed uniform pseudo-random number generator. ACM Transactions on Modeling and Computer Simulation, 8, 3 (1998), URL http://dx.doi.org/10.1145/272991.272995.

$\left[\mathrm{MPB}^{+}\right.$05] Melko R.G., Paramekanti A., Burkov A.A., Vishwanath A., Sheng D.N. and Balents L., Supersolid Order from Disorder: Hard-Core Bosons on the Triangular Lattice. Physical Review Letters, 95, 127207 (2005), URL http://dx.doi.org/10.1103/PhysRevLett.95. 127207. 
[MR11] Mecklenburg M. and Regan B., Spin and the Honeycomb Lattice: Lessons from Graphene. Physical Review Letters, 106, 116803 (2011), URL http://dx.doi.org/10. 1103/PhysRevLett.106.116803.

[MRR $\left.{ }^{+} 53\right]$ Metropolis N., Rosenbluth A.W., Rosenbluth M.N., Teller A.H. and Teller E., Equation of State Calculations by Fast Computing Machines. The Journal of Chemical Physics, 21, 1087 (1953), URL http://dx.doi.org/10.1063/1.1699114.

[MS00] Mascagni M. and Srinivasan A., Algorithm 806: SPRNG: a scalable library for pseudorandom number generation. ACM Transactions on Mathematical Softwarem, 26, 436 (2000), URL http://dx.doi.org/10.1145/358407.358427.

[MS01] Moessner R. and Sondhi S.L., Resonating Valence Bond Phase in the Triangular Lattice Quantum Dimer Model. Physical Review Letters, 86, 1881 (2001), URL http://dx.doi. org/10.1103/PhysRevLett.86.1881.

[Mus10] Mussardo G., Statistical field theory, (Oxford University Press, Oxford, 2010), 1st edition.

[MW66] Mermin N.D. and Wagner H., Absence of Ferromagnetism or Antiferromagnetism in Oneor Two-Dimensional Isotropic Heisenberg Models. Physical Review Letters, 17, 1133 (1966), URL http://dx.doi.org/10.1103/PhysRevLett.17.1133.

[NC08] Ng K.K. and Chen Y.C., Supersolid phases in the bosonic extended Hubbard model. Physical Review B, 77, 052506 (2008), URL http://dx.doi.org/10.1103/PhysRevB.77.052506.

[NGE98] Nersesyan A.A., Gogolin A.O. and Eßler F.H.L., Incommensurate Spin Correlations in Spin1/2 Frustrated Two-Leg Heisenberg Ladders. Physical Review Letters, 81, 910 (1998), URL http://dx.doi.org/10.1103/PhysRevLett.81.910.

[Nig77] Nightingale M., Non-universality for Ising-like spin systems. Physics Letters A, 59, 486 (1977), URL http://dx.doi.org/10.1016/0375-9601(77)90665-X.

[Nor11] Norman M.R., The challenge of unconventional superconductivity. Science, 332, 196 (2011), URL http://dx.doi.org/10.1126/science.1200181.

[OB78] Oitmaa J. and Betts D.D., The ground state of two quantum models of magnetism. Canadian Journal of Physics, 56, 897 (1978), URL http://dx.doi.org/10.1139/p78-120.

[OHZ06] Oitmaa J., Hamer C. and Zheng W., Series Expansion Methods, (Cambridge University Press, Cambridge, 2006), 1st edition.

[Oit81] Oitmaa J., The square-lattice Ising model with first and second neighbour interactions. Journal of Physics A: Mathematical and General, 14, 1159 (1981), URL http://dx.doi.org/ $10.1088 / 0305-4470 / 14 / 5 / 035$.

[ONAKT07] Okamoto Y., Nohara M., Aruga-Katori H. and Takagi H., Spin-Liquid State in the S=1/2 Hyperkagome Antiferromagnet $\mathrm{Na}_{4} \mathrm{Ir}_{3} \mathrm{O}_{8}$. Physical Review Letters, 99, 137207 (2007), URL http://dx.doi.org/10.1103/PhysRevLett.99.137207.

[Ons44] Onsager L., Crystal Statistics. I. A Two-Dimensional Model with an Order-Disorder Transition. Physical Review, 65, 117 (1944), URL http://dx.doi.org/10.1103/PhysRev. 65. 117.

[OP99] Ogburn W.R. and Preskill J., Topological Quantum Computation, in Quantum Computing and Quantum Communications (edited by C. Williams), volume 1509 of Lecture Notes in Computer Science, pages 341-356, (Springer, Berlin, 1999), URL http://dx.doi.org/10. 1007/3-540-49208-9_31.

[OS11] Oitmaa J. and Singh R., Phase diagram of the $J_{1}-J_{2}-J_{3}$ Heisenberg model on the honeycomb lattice: A series expansion study. Physical Review B, 84, 094424 (2011), URL http://dx.doi.org/10.1103/PhysRevB.84.094424.

[OW96] Oitmaa J. and Weihong Z., Series expansion for the $J_{1}-J_{2}$ Heisenberg antiferromagnet on a square lattice. Physical Review B, 54, 3022 (1996), URL http://dx.doi.org/10.1103/ PhysRevB.54.3022.

[Par98] Parlett B.N., The Symmetric eigenvalue problem, (SIAM, Philadelphia, 1998), 1st edition.

[PC87] Pollock E.L. and Ceperley D.M., Path-integral computation of superfluid densities. Physical Review B, 36, 8343 (1987), URL http://dx.doi.org/10.1103/PhysRevB.36.8343. 
[RAT11] Reuther J., Abanin D.A. and Thomale R., Magnetic order and paramagnetic phases in the quantum $J_{1}-J_{2}-J_{3}$ honeycomb model. Physical Review B, 84, 014417 (2011), URL http://dx.doi.org/10.1103/PhysRevB.84.014417.

$\left[\mathrm{RDS}^{+} 10\right] \quad$ Richter J., Darradi R., Schulenburg J., Farnell D.J.J. and Rosner H., Frustrated spin-1/2 $J_{1}-J_{2}$ Heisenberg ferromagnet on the square lattice studied via exact diagonalization and coupled-cluster method. Physical Review B, 81, 174429 (2010), URL http://dx.doi.org/ 10.1103/PhysRevB.81.174429.

$\left[\mathrm{RFC}^{+} 04\right]$ Roscilde T., Feiguin A., Chernyshev A., Liu S. and Haas S., Anisotropy-Induced Ordering in the Quantum $J_{1}-J_{2}$ Antiferromagnet. Physical Review Letters, 93, 017203 (2004), URL http://dx.doi.org/10.1103/PhysRevLett.93.017203.

[RMP09] Ralko A., Mambrini M. and Poilblanc D., Generalized quantum dimer model applied to the frustrated Heisenberg model on the square lattice: Emergence of a mixed columnarplaquette phase. Physical Review B, 80, 184427 (2009), URL http://dx.doi.org/10. 1103/PhysRevB . 80.184427.

[RS09] Richter J. and Schulenburg J., The spin-1/2 $J_{1}-J_{2}$ Heisenberg antiferromagnet on the square lattice: Exact diagonalization for $N=40$ spins. The European Physical Journal B, 73, 117 (2009), URL http://dx.doi.org/10.1140/epjb/e2009-00400-4.

[RSH04] Richter J., Schulenburg J. and Honecker A., Quantum Magnetism in Two Dimensions: From Semi-Classical Néel Order to Magnetic Disorder, in Quantum Magnetism (edited by U. Schollwöck, J. Richter, D.J.J. Farnell and R.F. Bishop), volume 645 of Lecture Notes in Physics, chapter 2, page 85, (Springer, Berlin, 2004), URL http://www.springerlink. com/content/ccv22wcn1jnrxfhu/.

[Sac99] Sachdev S., Quantum Phase Transitions, (Cambridge University Press, Cambridge, 1999), 1st edition.

[Sac04] Sachdev S., Quantum Phases and Phase Transitions of Mott Insulators, in Quantum Magnetism (edited by U. Schollwöck, J. Richter, D.J.J. Farnell and R.F. Bishop), volume 645 of Lecture Notes in Physics, chapter 9, pages 381-432, (Springer, Berlin, 2004), URL http:// ww. springerlink. com/content/twlelqkygyk5mhur/.

[Sac12] Sachdev S., Quantum Phase Transitions of Antiferromagnets and the Cuprate Superconductors, in Modern Theories of Many-Particle Systems in Condensed Matter Physics (edited by D.C. Cabra, A. Honecker and P. Pujol), volume 843 of Lecture Notes in Physics, chapter 1, pages 1-51, (Springer, Berlin, 2012), URL http://dx.doi.org/10. 1007/978-3-642-10449-7.

[San92] Sandvik A.W., A generalization of Handscomb's quantum Monte Carlo scheme-application to the 1D Hubbard model. Journal of Physics A: Mathematical and General, 25, 3667 (1992), URL http://dx.doi.org/10.1088/0305-4470/25/13/017.

[San12] Sandvik A.W., Finite-size scaling in two-dimensional valence-bond-solids. Physical Review B, 85, 134407 (2012), URL http://dx.doi.org/10.1103/PhysRevB.85.134407.

[SB04] Starykh O. and Balents L., Dimerized Phase and Transitions in a Spatially Anisotropic Square Lattice Antiferromagnet. Physical Review Letters, 93, 127202 (2004), URL http:// dx.doi.org/10.1103/PhysRevLett.93.127202.

[Sch] Schulenburg J., Spinpack, URL http://www-e.uni-magdeburg.de/jschulen/spin/ index.html.

[Sch00] Schwabl F., Statistische Mechanik, (Springer, Berlin, 2000), 1st edition.

[Sch03] Schulenburg J., Einfluss der Gitterstruktur auf den Grundzustand von $s=1 / \mathcal{L}_{-}$ Quantenspinsystemen, Doktorarbeit, Otto-von-Guericke-Universität Magdeburg (2003), URL http://www-e.uni-magdeburg.de/jschulen/diss/diss.pdf.

[Sch04] Schmid G., Phase transitions of hard-core bosons, Doktorarbeit, Swiss Federal Institute of Technology Zürich (2004).

[Sch11] Schollwöck U., The density-matrix renormalization group in the age of matrix product states. Annals of Physics, 326, 96 (2011), URL http://dx.doi.org/10.1016/j.aop.2010.09. 012. 
[Sel88] Selke W., The ANNNI model - Theoretical analysis and experimental application. Physics Reports, 170, 213 (1988), URL http://dx.doi.org/10.1016/0370-1573(88)90140-8.

[Sel06] Selke W., Critical Binder cumulant of two-dimensional Ising models. The European Physical Journal B, 51, 223 (2006), URL http://dx.doi.org/10.1140/epjb/e2006-00209-7.

[SFB05] Starykh O., Furusaki A. and Balents L., Anisotropic pyrochlores and the global phase diagram of the checkerboard antiferromagnet. Physical Review B, 72, 094416 (2005), URL http://dx.doi.org/10.1103/PhysRevB.72.094416.

[SH99] Sandvik A. and Hamer C., Ground-state parameters, finite-size scaling, and low-temperature properties of the two-dimensional $S=1 / 2 X Y$ model. Physical Review B, 60, 6588 (1999), URL http://dx.doi.org/10.1103/PhysRevB.60.6588.

[SK79] Swendsen R. and Krinsky S., Monte Carlo Renormalization Group and Ising Models with $n \gtrsim 2$. Physical Review Letters, 43, 177 (1979), URL http://dx.doi.org/10.1103/ PhysRevLett.43.177.

[SK91] Sandvik A. and Kurkijärvi J., Quantum Monte Carlo simulation method for spin systems. Physical Review B, 43, 5950 (1991), URL http://dx.doi.org/10.1103/PhysRevB.43. 5950.

[SM99] Sato A. and Matsubara F., Equilibrium properties of an axial next-nearest-neighbor Ising model in two dimensions. Physical Review B, 60, 10316 (1999), URL http://dx.doi.org/ 10.1103/PhysRevB.60.10316.

[SMK $\left.{ }^{+} 03\right]$ Shimizu Y., Miyagawa K., Kanoda K., Maesato M. and Saito G., Spin Liquid State in an Organic Mott Insulator with a Triangular Lattice. Physical Review Letters, 91, 107001 (2003), URL http://dx.doi.org/10.1103/PhysRevLett.91.107001.

[SO83] Suzuki K. and Okamoto R., Degenerate Perturbation Theory in Quantum Mechanics. Progress of Theoretical Physics, 70, 439 (1983), URL http://dx.doi.org/10.1143/PTP. 70.439 .

$\left[\mathrm{SOL}^{+}{ }^{+11} \quad\right.$ Struck J., Olschläger C., Le Targat R., Soltan-Panahi P., Eckardt A., Lewenstein M., Windpassinger P. and Sengstock K., Quantum Simulation of Frustrated Classical Magnetism in Triangular Optical Lattices. Science, 333, 996 (2011), URL http://dx.doi.org/10.1126/ science.1207239.

[SRT00] Simon P. and Ricci-Tersenghi F., Coupled Ising models with disorder. Journal of Physics A: Mathematical and General, 33, 5985 (2000), URL http://dx.doi.org/10.1088/ $0305-4470 / 33 / 34 / 304$.

[SS02] Syljuåsen O.F. and Sandvik A.W., Quantum Monte Carlo with directed loops. Physical Review E, 66, 046701 (2002), URL http://dx.doi.org/10.1103/PhysRevE.66.046701.

[SS10] Si Q. and Steglich F., Heavy fermions and quantum phase transitions. Science, 329, 1161 (2010), URL http://dx.doi.org/10.1126/science.1191195.

[SSB08] Schnyder A., Starykh O. and Balents L., Spatially anisotropic Heisenberg kagome antiferromagnet. Physical Review B, 78, 174420 (2008), URL http://dx.doi.org/10.1103/ PhysRevB.78.174420.

[SSC02] Sengupta P., Sandvik A. and Campbell D., Bond-order-wave phase and quantum phase transitions in the one-dimensional extended Hubbard model. Physical Review B, 65, 155113 (2002), URL http://dx.doi.org/10.1103/PhysRevB.65.155113.

[Ste84] Stewart G., Heavy-fermion systems. Reviews of Modern Physics, 56, 755 (1984), URL http://dx.doi.org/10.1103/RevModPhys.56.755.

[Suz74] Suzuki M., New Universality of Critical Exponents. Progress of Theoretical Physics, 51, 1992 (1974), URL http://dx.doi.org/10.1143/PTP.51.1992.

[SW87] Swendsen R. and Wang J.S., Nonuniversal critical dynamics in Monte Carlo simulations. Physical Review Letters, 58, 86 (1987), URL http://dx.doi.org/10.1103/PhysRevLett. 58.86.

[Tsv95] Tsvelik A.M., Quantum field theory in condensed matter physics, (Cambridge University Press, Cambridge, 1995), 1st edition. 
[Tsv01] Tsvelik A., A mechanism for ferrimagnetism and incommensurability in one-dimensional systems. Nuclear Physics B, 612, 479 (2001), URL http://dx.doi.org/10.1016/ S0550-3213(01)00334-0.

[VB81] Villain J. and Bak P., Two-dimensional ising model with competing interactions: floating phase, walls and dislocations. Journal de Physique, 42, 657 (1981), URL http://dx.doi. org/10.1051/jphys:01981004205065700.

[VBCC80] Villain J., Bidaux R., Carton J.P. and Conte R., Order as an effect of disorder. Journal de Physique, 41, 1263 (1980), URL http://dx.doi.org/10.1051/jphys: 0198000410110126300.

[Wen91] Wen X., Mean-field theory of spin-liquid states with finite energy gap and topological orders. Physical Review B, 44, 2664 (1991), URL http://dx.doi.org/10.1103/PhysRevB.44. 2664.

[WGWV11] Wang L., Gu Z.C., Wen X.G. and Verstraete F., Possible spin liquid state in the spin$1 / 2 J_{1}-J_{2}$ antiferromagnetic Heisenberg model on square lattice: A tensor product state approach. preprint: 1112.3331 (2011), URL http://arxiv.org/abs/1112.3331.

[Whi92] White S.R., Density matrix formulation for quantum renormalization groups. Physical Review Letters, 69, 2863 (1992), URL http://dx.doi.org/10.1103/PhysRevLett.69.2863.

[Wie93] Wiese U., Bosonization and cluster updating of lattice fermions. Physics Letters B, 311, 235 (1993), URL http://dx.doi.org/10.1016/0370-2693(93)90561-U.

[Wol89] Wolff U., Collective Monte Carlo Updating for Spin Systems. Physical Review Letters, 62, 361 (1989), URL http://dx.doi.org/10.1103/PhysRevLett.62.361.

[WT05] Wessel S. and Troyer M., Supersolid Hard-Core Bosons on the Triangular Lattice. Physical Review Letters, 95, 127205 (2005), URL http://dx.doi.org/10.1103/PhysRevLett.95. 127205.

[YHW11] Yan S., Huse D.A. and White S.R., Spin-liquid ground state of the $S=1 / 2$ kagome Heisenberg antiferromagnet. Science, 332, 1173 (2011), URL http://dx.doi.org/10. $1126 /$ science. 1201080.

[YLMS10] Yang H.Y., Läuchli A.M., Mila F. and Schmidt K.P., Effective Spin Model for the SpinLiquid Phase of the Hubbard Model on the Triangular Lattice. Physical Review Letters, 105, 267204 (2010), URL http://dx.doi.org/10.1103/PhysRevLett.105. 267204.

[YS11] Yang H.Y. and Schmidt K.P., Effective models for gapped phases of strongly correlated quantum lattice models. Europhysics Lettersz, 94, 17004 (2011), URL http://dx.doi. org/10.1209/0295-5075/94/17004.

[Zam86] Zamolodchikov A.B., "Irreversibily" of the flux of the renormalization group in a 2D field theory. Journal of Experimental and Theoretical Physics Letters, 43, 730 (1986), URL http://www.jetpletters.ac.ru/ps/1413/article_21504.shtml.

[ZC11] Zhang K. and Charbonneau P., Monte Carlo approach for studying microphases applied to the axial next-nearest-neighbor Ising and the Ising-Coulomb models. Physical Review B, 83, 214303 (2011), URL http://dx.doi.org/10.1103/PhysRevB.83.214303.

[ZCS ${ }^{+}$06] Zaanen J., Chakravarty S., Senthil T., Anderson P., Lee P., Schmalian J., Imada M., Pines D., Randeria M., Varma C., Vojta M. and Rice M., Towards a complete theory of high $T_{C}$. Nature Physics, 2, 138 (2006), URL http://dx.doi.org/10.1038/nphys253. 


\section{Lebenslauf}

Name: Ansgar Kalz

Staatsangehörigkeit: deutsch

25.02.1983 geboren in Berlin

09/1989 - 06/2002 Besuch von Grundschule und Gymnasium (Canisius Kolleg, Berlin-Tiergarten)

06/2002 Erwerb der allgemeinen Hochschulreife

10/2003 - 10/2008 Studium an der Georg-August-Universität Göttingen im Studiengang Physik

07/2005 Erwerb des Vordiploms im Studiengang Physik an der Universität Göttingen

08/2005 - 07/2006 Studium der Physik an der Universidad Nacional de Colombia in Bogotá

10/2008 Erwerb des Hochschulgrades Diplom-Physiker an der Universität Göttingen

01/2009 - 02/2012 Arbeit an der vorliegenden Dissertation bei PD Dr. Andreas Honecker an der Universität Göttingen

09/2010 - 10/2010 Auslandsaufenthalt im Rahmen der Promotion an der Universidad Nacional de La Plata (Argentinien) 